Portland State University

PDXScholar

Spring 5-29-2015

\title{
Assessing Unique Core Values with the Competing Values Framework: The CCVI Technique for Guiding Organizational Culture Change
}

Anthony John Santoriello

Portland State University

Follow this and additional works at: https://pdxscholar.library.pdx.edu/open_access_etds

Part of the Work, Economy and Organizations Commons Let us know how access to this document benefits you.

\section{Recommended Citation}

Santoriello, Anthony John, "Assessing Unique Core Values with the Competing Values Framework: The CCVI Technique for Guiding Organizational Culture Change" (2015). Dissertations and Theses. Paper 2315.

https://doi.org/10.15760/etd.2312

This Dissertation is brought to you for free and open access. It has been accepted for inclusion in Dissertations and Theses by an authorized administrator of PDXScholar. For more information, please contact pdxscholar@pdx.edu. 
Assessing Unique Core Values with the Competing Values Framework:

The CCVI Technique for Guiding Organizational Culture Change

by

Anthony John Santoriello

A dissertation submitted in partial fulfillment of the requirements for the degree of

Doctor of Philosophy

in

Systems Science

Dissertation Committee:

Wayne Wakeland, Chair

David E. Hall, Co-Chair

Susan E. Marcus

Alan Cabelly

Portland State University

2015 


\section{Abstract}

Extensive research suggests organizations have unique guiding principles, called core values, which play a central role in strategic decision making, sustaining highperformance cultures, and guiding organizational culture change. Although the Competing Values Framework (CVF) has been widely used to identify a standardized set of core values, it has not been used to identify unique core values at a given organizations. Unique core values help to distinguish organizations and drive market success.

The present research focused on development of a technique to extend the application of the CVF to identify the core values unique to a given organization. The CVF-based Core Values Identification Technique (CCVI) was developed and empirically tested at three companies. Data collection methods included semi-structured interviews, review of documents, participant observation and the standard CVF-based Organizational Culture Assessment Instrument (OCAI). The data analysis employed grounded theory methods in conjunction with the standard OCAI analysis.

The primary research result is the iteratively developed and empirically tested tool, the CCVI Technique. Secondary research results include the identification of unique core values at each of the three participating companies. Lessons learned and the rationale for making modifications to the technique based on the case studies, along with best practices for utilization and opportunities for informing organizational change efforts are discussed. 


\section{Acknowledgements}

I acknowledge with sincere gratitude the support and suggestions of my committee members: Wayne Wakeland, David E. Hall, Susan E. Marcus, and Alan Cabelly. A dissertation is a long journey and I thank you all for guiding me with wisdom and encouragement. I also cannot thank my wife, Sarah, enough for her patience and support through this process.

Finally, my gratitude goes out to the three companies who opened their doors for this research. May you continue to be successful! 


\section{Table of Contents}

Acknowledgements...

2.1 Theoretical Frameworks Employed .............................................................. 5

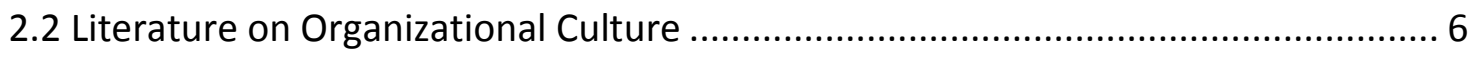

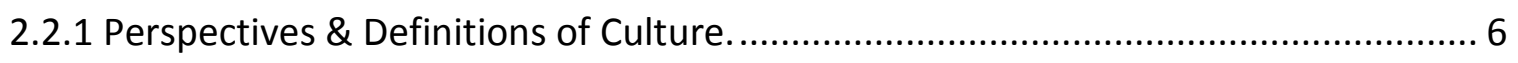

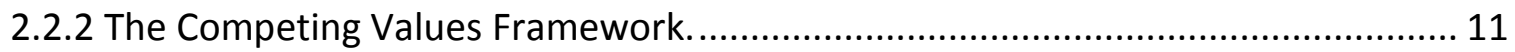

2.2.3 Diagnosing Organizational Culture. ............................................................. 13

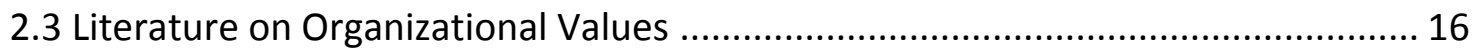

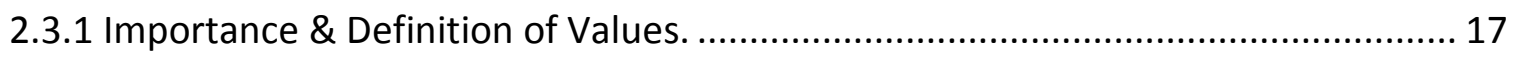

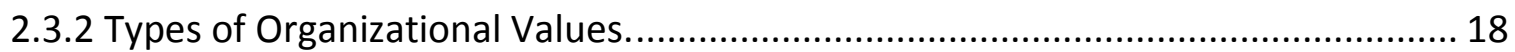

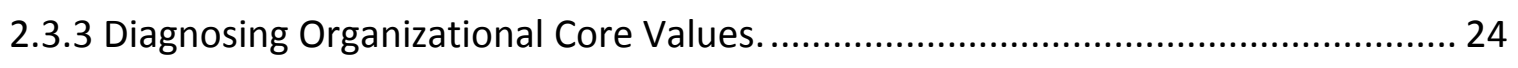

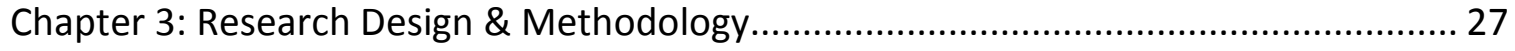

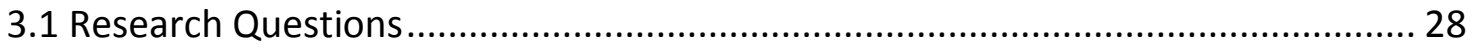

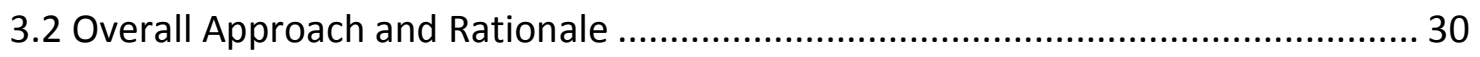

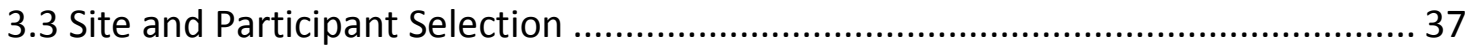

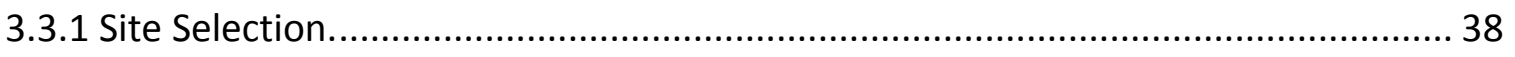

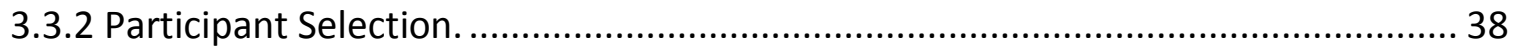

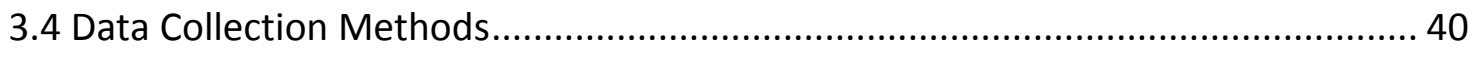

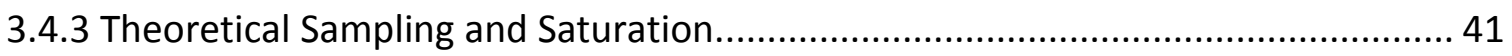

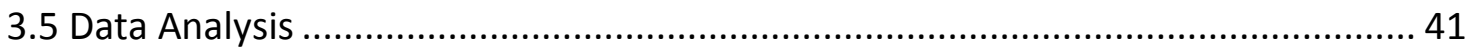


iv

3.6 How Trustworthiness of Data Was Maintained ............................................ 43

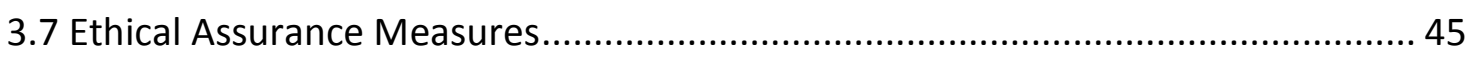

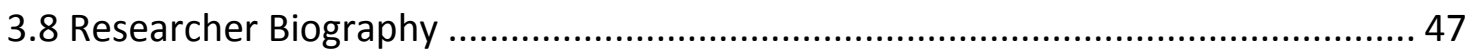

Chapter 4: Primary Research Results: The CCVI Technique ........................................ 49

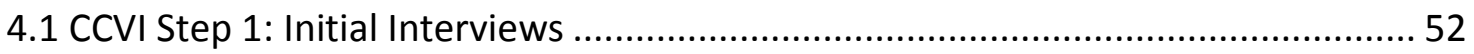

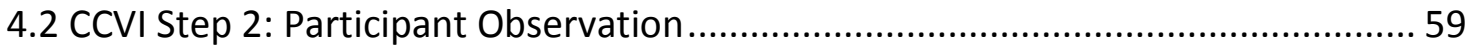

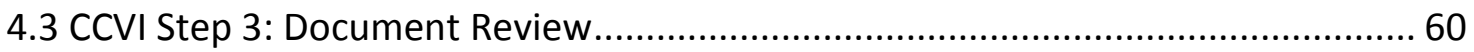

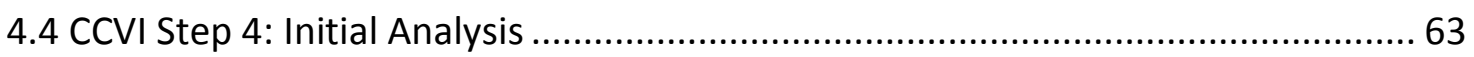

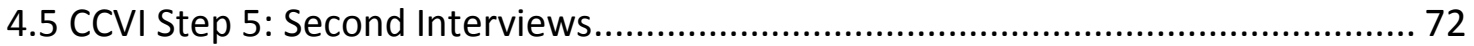

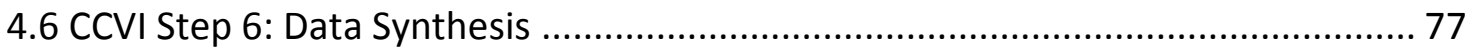

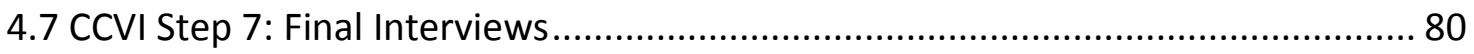

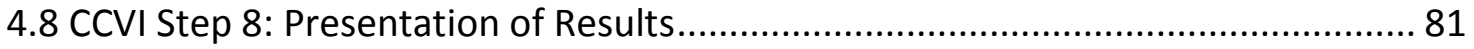

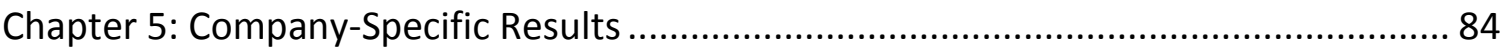

5.1 Introduction to Company A Case Study Results................................................ 84

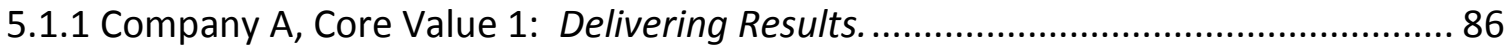

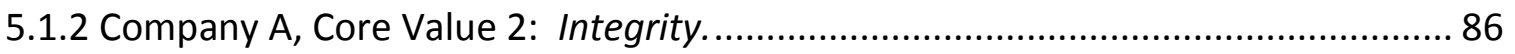

5.1.3 Company A, Core Value 3: Developing our People. ............................................. 87

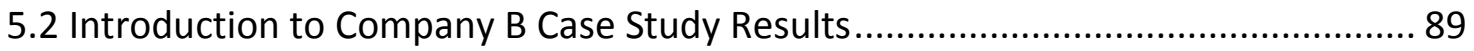

5.2.1 Company B, Core Value 1: Do What You Love................................................... 92

5.2.2 Company B, Core Value 2: Problem Solving DNA ............................................. 93

5.2.3 Company B, Core Value 3: A Culture of Integrity, Maturity \& Respectfulness. ...... 94

5.3 Introduction to Company C Case Study Results............................................... 95

5.3.1 Company C, Core Value 1: The Company C Winning Mindset.............................. 98

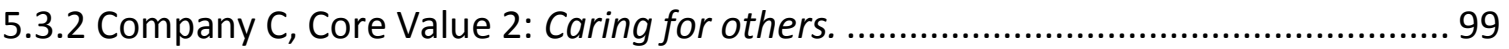

5.3.3 Company C, Core Value 3: Balanced Analytical \& Emotional Intelligence. ........... 100

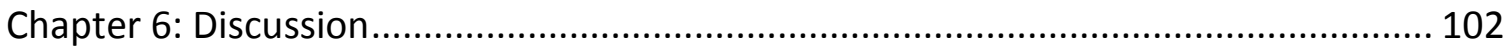

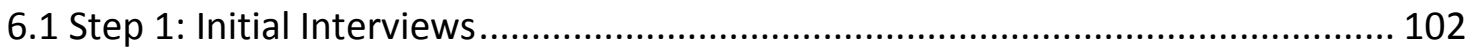

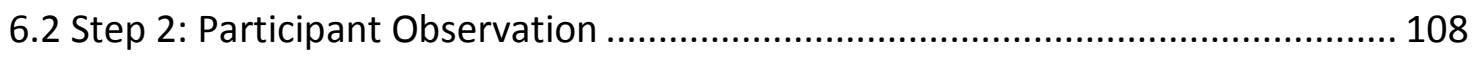

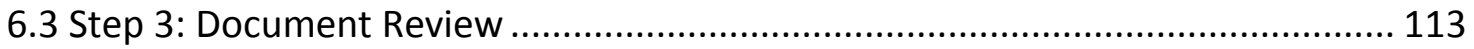

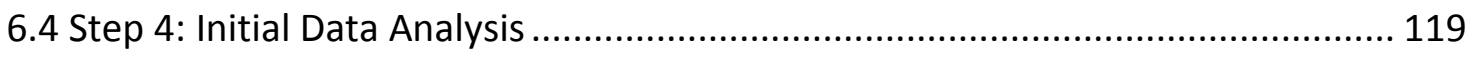




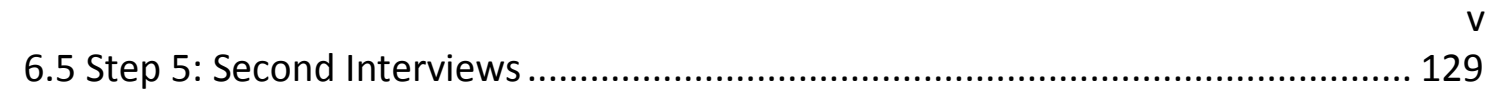

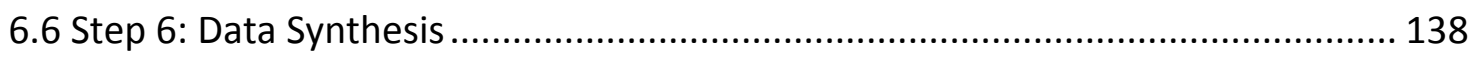

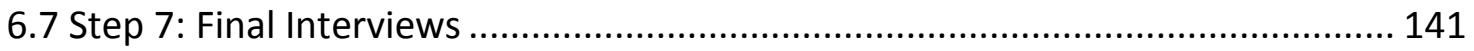

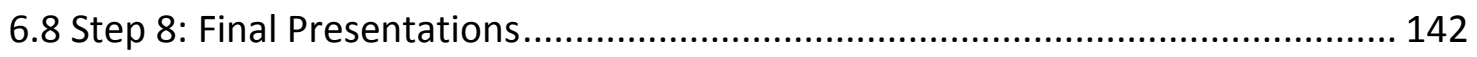

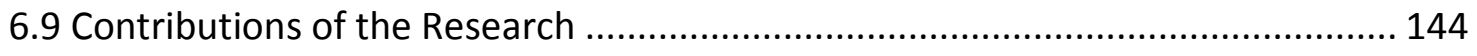

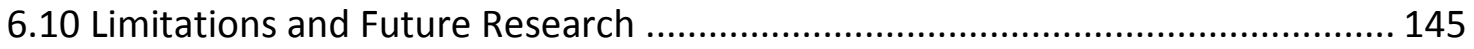

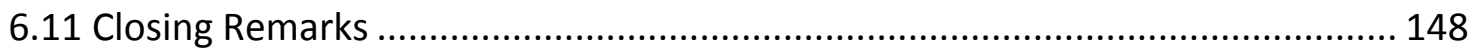

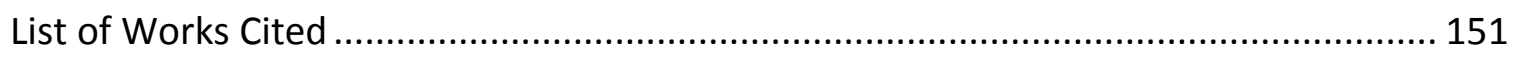

Appendix A: Study Participant Recruitment Letter ............................................... 157

Appendix B: Owner Informed Consent Request................................................... 159

Appendix C: Interviewee Informed Consent Request ............................................. 161

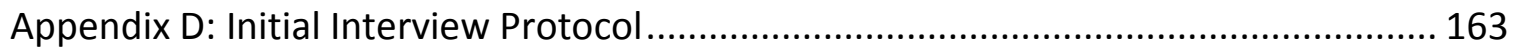

Appendix E: Demographic Questionnaire........................................................... 166

Appendix F: Initial Interview Worksheet ............................................................ 168

Appendix G: Organizational Culture Assessment Instrument \& Scoring Sheet ............. 169

Appendix H: Participant Observation Field Guide ................................................... 174

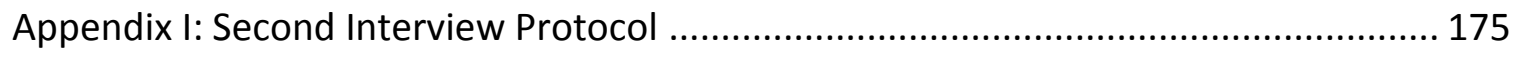

Appendix J: Overall Culture Assessment Template ................................................ 177

Appendix K: Itemized Culture Assessment Template ................................................ 178

Appendix L: Types of Organizational Values Chart ................................................... 180

Appendix M: Core Values Identification Form (Example) ........................................ 181

Appendix N: Value-Behavior Association Form (Example) ......................................... 182

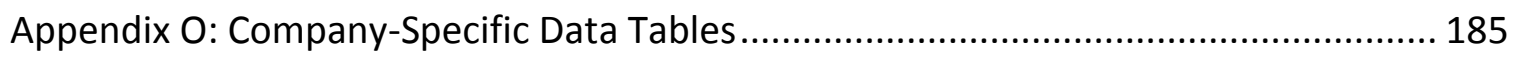




\section{List of Tables}

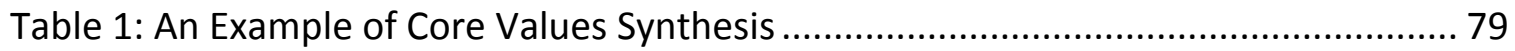

Table 2: Company B Core Value Candidates................................................................. 91

Table 3: Initial Semi-Structured Interview Question 1 Discussion Table....................... 103 


\section{List of Figures}

Figure 1: The Competing Values Framework............................................................. 2

Figure 2: Four Popular Perspectives on Organizational Culture....................................... 7

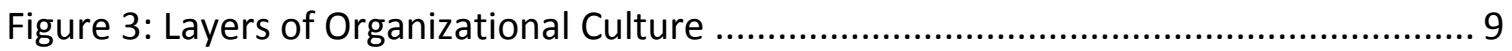

Figure 4: The First Question of the Organizational Culture Assessment Instrument....... 15

Figure 5: Culture profile example generated from an OCAI assessment ....................... 16

Figure 6: Southwest Airlines Core Values and behaviors associated with each .............. 20

Figure 7: The Meta-Methodology for this research .................................................. 30

Figure 8: The CCVI Technique Overview (CVF-based Core Values Identification)............ 49

Figure 9: Flowchart for CCVI Technique Step 1: Initial Interview Protocol.. .................... 53

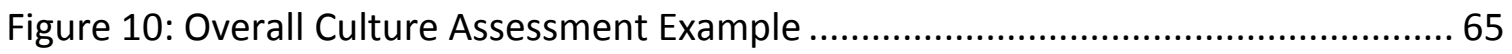

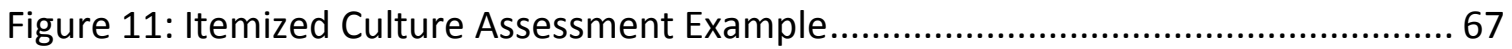

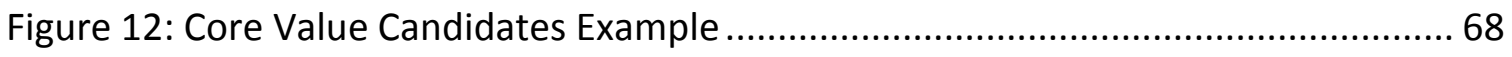

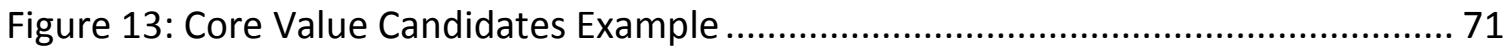

Figure 14: Flowchart for CCVI Technique Step 5: Second Interview Protocol Overview. 73

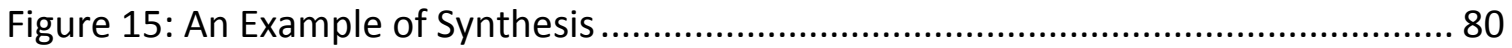

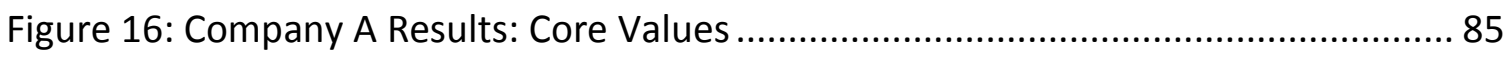

Figure 17: Company B Results: Core Values ............................................................... 90

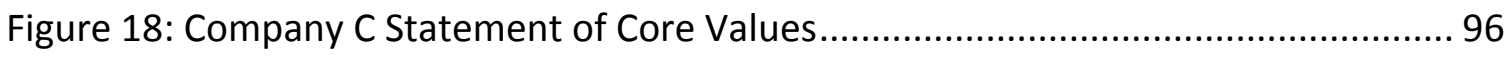

Figure 19: Company A Combined OCAI results............................................................. 108

Figure 20: Company A Preexisting Statement of Core Values...................................... 116

Figure 21: Company A Complete Values Statement...................................................... 118

Figure 22: Snapshot of a complete Overall Culture Assessment form .......................... 121

Figure 23: Snapshot of a complete Itemized Culture Assessment form ...................... 123

Figure 24: Snapshot of a complete Company-Wide Culture Assessment form ............ 125

Figure 25: The Typical Conceptual Mapping from Data to Core Value Candidates ....... 128

Figure 26: Minor Worksheet Improvements. .............................................................. 133

Figure 27: Overview of technical changes to CVI and V-BA forms .............................. 136

Figure 28: Company B Core Values Identification Form Feedback Synthesis ................ 140 
"Core values are the deeply ingrained principles that guide all of a company's actions; they serve as its cultural corner stones... They are the source of a company's distinctiveness." - Patrick Lencioni

\section{Chapter 1: Overview}

\subsection{Introduction}

The long-term economic performance of organizations is significantly impacted by corporate culture (Kotter, 1992). Because cultures are unique and difficult to copy, high-performing cultures are a source of sustainable competitive advantage in business (Barney, 1986). However, significant performance problems have also been attributed to various deficiencies in organizational culture (Hogan \& Coote, 2014). Assessing and improving organizational culture is important because fierce competition on a global scale continues to drive up performance requirements on modern firms.

The Organizational Culture Assessment Instrument (OCAI) is the most extensively used, tested and validated instrument for assessing organizational culture (Cameron \& Quinn, 2011). The OCAl is standardized and simple to implement with only six questions. The OCAl is used worldwide by HR professionals and consultants to assist with identifying organizational values and guiding organizational change efforts because it offers a reliable mechanism for identifying characteristic organizational values. The purpose of the OCAl is to assess an organization's culture and characteristic values, based on the Competing Values Framework (CVF) (see Figure 1, below). The Competing Values Framework is the most widely utilized framework for assessing organizational 
culture in the world (Cameron \& Quinn, 2011). According to Cameron and Quinn,

"More than 80 percent of the several thousand organizations we have studied have been characterized by one or more of the culture types identified by the [Competing Values] framework. Those that do not have a dominant culture type either tend to be unclear about their culture or emphasize the four different culture types nearly equally" (Cameron \& Quinn, 2011, p. 52).

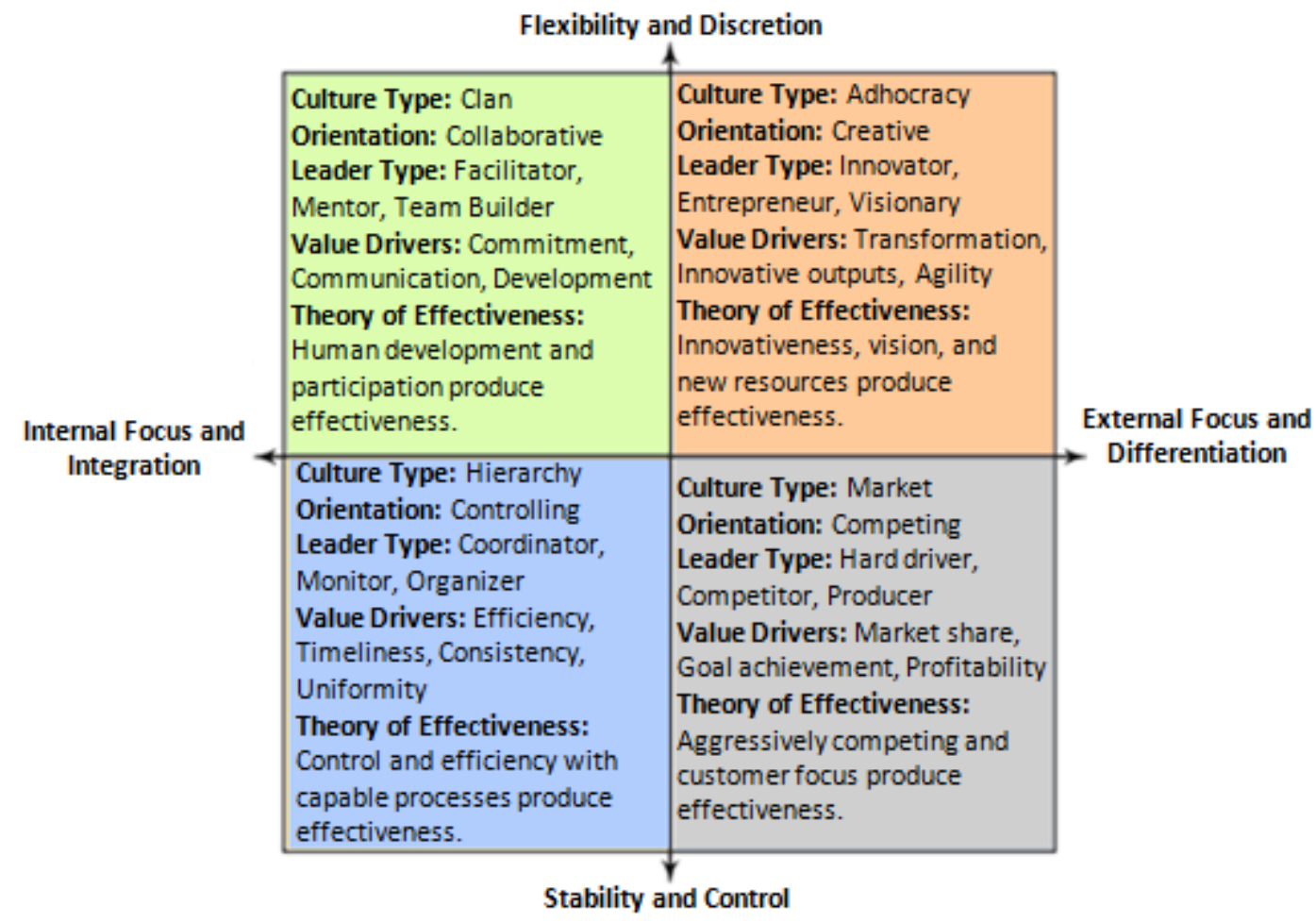

Figure 1: The Competing Values Framework. (Source: Adapted from Fig 3.2, The Competing Values of Leadership, Effectiveness, and Organizational Theory, Cameron \& Quinn, 2011, p.53)

It is known that standardized instruments tend to stress common features that exist across many organizations, but often do not help investigators discover unique patterns, values or cultural features within particular organizations or their subcultures 
(Harrison \& Shirom, 1999). Similarly, while the OCAI does serve to provide a baseline for assessing organizational culture, a major drawback is that the results, being standardized, fail to penetrate or capture what is unique about the organization.

In their book Built to Last: Successful Habits of Visionary Companies, Collins and Porras (1994) made a compelling case that the best companies have and adhere to a unique set of guiding principles called core values. Since 1994, most corporations and nearly all Fortune 100 companies have published values statements in attempting to publicly clarify their most important (core) values. While core values are essential to organizational performance, questions have been raised about the quality of many of these published values statements. Organizational scholar and consultant Patrick Lencioni describes most of the published value statements he has seen as "bland, toothless, or just plain dishonest" (Lencioni, 2002, p. 5). Poor quality values statements are more than a missed opportunity; they are also harmful to companies. They engender mistrust and cynicism in the company culture, and fail to "set a company apart from the competition by clarifying its identity" (Lencioni, 2002, p. 6). The effective assessment of organizational core values is the important first step in avoiding these issues.

Core values are the most important values in an organization. They are those values with more influence on behavior or evaluation than most other values in a system (Pant \& Lachman, 1998; Van Rekom, Van Riel, \& Wierenga, 2006). Core values, unique to each firm, play a central role in guiding strategic decision making (Marcus, 2004) and aligning corporate strategy and culture. Leading OCAI scholars acknowledge 
the importance of core values, stating that "Having a diagnostic instrument to identify core organizational culture values can be an especially useful tool in the effective management of organizational change" (Cameron \& Quinn, 2011, p. 167). Surprisingly, the OCAI has not been extended to identify the unique core values which characterize organizations, despite the importance of unique core values in anchoring successful change initiatives and sustaining high-performance culture.

\subsection{Research Purpose}

The purpose of this research project was to develop a technique that extends the application of the Competing Values Framework, beyond the OCAl's assessment of archetypal organizational values, to identify unique organizational core values. The primary result is the CCVI Technique (CVF-based Core Values Identification), which allows companies to obtain greater insight into their cultural values to improve guidance for change initiatives and sustain high-performance culture. The CCVI Technique, described in detail in Chapter 4, employs Grounded Theory (GT) data analysis techniques to assess organizational core values from data collected at a target company. GT is an iterative process, structured to allow themes, topics and insights to emerge from collected data. To achieve the purpose of the research project, three case studies, one at each of three subject companies, were completed to provide the laboratory for developing a technique for identifying organizational core values. A secondary purpose of this proposed research, while iteratively developing the proposed technique, was to identify unique organizational core values at each of the three subject companies (described in detail in Chapter 5). 


\section{Chapter 2: Literature Review}

The review of literature here focuses on the theoretical frameworks employed in this research, as well as understanding organizational culture and values, and assessing organizational culture and values. Culture and values are treated separately in sections 2.2 and 2.3, respectively, and post-analysis literature review is integrated with the Chapter 6 discussion of results.

\subsection{Theoretical Frameworks Employed}

This research utilized three key theoretical perspectives: symbolic interaction theory, organizational culture theory, and the competing values framework. Each of these theoretical perspectives will be briefly described in turn, followed by a more in depth literature review of organizational culture and the competing values framework in the next section.

Symbolic interactionism, the guiding theoretical framework for this research, is a sociological perspective which acknowledges that social meaning is "derived from, or arises out of, the social interaction that one has with others and the society" (Blumer, 1986, p. 2). Because this research seeks to identify workers' unique, subjective understandings of their workplace cultures, a theory of social meaning is necessary. Symbolic interactionism theory will help us understand that the diverse behaviors and multiple perspectives of workers on company culture and values are reflections of their unique understandings of their workplace cultures. 
Organizational culture theory is a framework for understanding organizations through a cultural lens with a focus on values, attitudes, behaviors and beliefs of members (Bowditch, Buono, \& Stewart, 2007). Culture is described on a continuum between "objective" and "subjective" in this framework, proceeding from cultural artifacts through patterns of behavior, behavioral norms, and shared values, to fundamental beliefs and assumptions. This framework was used in this research as an organizing principle for understanding the culture at each subject company.

The Competing Values Framework (CVF) was chosen as an underlying framework for this research because is the most dominant framework for assessing organizational culture in the world (Cameron \& Quinn, 2011). Cameron and Quinn suggest the CVF has achieved this status because the four archetypes within the framework are congruent with "an underlying similarity in people at the deep psychological level of their cognitive processes" (Cameron \& Quinn, 2011, p. 37).

\subsection{Literature on Organizational Culture}

\subsubsection{Perspectives \& Definitions of Culture.}

The concept of culture began receiving serious attention from organizational scholars in the early 1980s (Cameron \& Quinn, 2011), and over the past three decades a complex and diverse body of theorizing and research in organizational culture has developed (Harrison, 2005). Hundreds of definitions have been identified for culture, helping to perpetuate the state of definitional ambiguity (Jahoda, 2012). The many definitions, models and proposed elements of culture vary in large part because of differing perspectives espoused by practitioners. The two most popular disciplinary 
foundations for organizational culture analysis are sociology (viewing organizations as having cultures) and anthropology (viewing organizations as cultures) (Cameron \& Quinn, 2011). A functional approach (assuming culture emerges from behavior) and semiotic approach (assuming culture exists within individuals' interpretations) also developed within each of these disciplines (Cameron \& Quinn, 2011). The juxtaposition of these approaches can be seen in Figure 2.

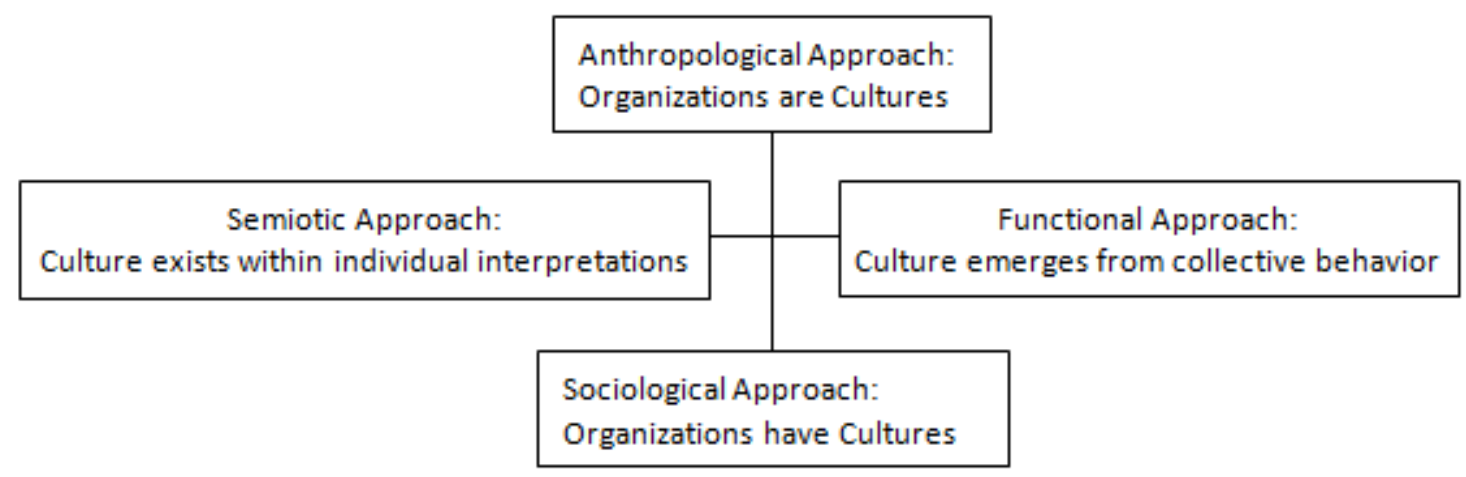

Figure 2: Four Popular Perspectives on Organizational Culture. Figure reflects the basic underlying assumptions characteristic of each approach.

The semiotic anthropological community tends to cite and propose a definition of culture as, "The set of important assumptions (often unstated) that members of a community share in common" (Sathe, 1985, p. 6). Notice that this definition focuses on assumptions as the defining feature of culture. This is the key to recognizing Sathe's perspective as anthropological. This quoted definition is also characterized as semiotic because the focus is on the assumptions of individuals ("members of a community").

The functional anthropological community approach will differ from the semiotic in that the focus is on collective assumptions, as seen in "Organizational culture is the pattern of basic assumptions that a given group has invented, discovered, or developed 
in learning to cope with its problems of external adaptation and internal integration" (Schein, 1992, p. 3). This explicit focus on assumptions is the key to recognizing anthropological definitions of culture, and the explicit focus on the function those assumptions play in serving the group is the key feature of the functional approach.

The functional sociological perspective on organizational culture is characterized by the assumption that culture is part of an organization and a focus on group cognition and behavior, as in this definition by Denison (1996, p. 624), "Culture refers to the deep structure of organizations, which is rooted in the values, beliefs, and assumptions held by organizational members. Meaning is established through socialization to a variety of identity groups that converge in the workplace." Denison's definition appears to acknowledge an interplay between groups and individuals, suggesting that functional and semiotic sociological perspectives are not mutually exclusive but rather exist on a continuum. The present research employs a functional sociological perspective, treating culture as an attribute of organizations which emerges from collective behavior.

Dimensions of organizational culture. A wide variety of dimensions have also been proposed to help organize theory and study of organizational culture. The variety of dimensions proposed for organizational culture has been no less diverse than definitions for the concept. A fairly comprehensive model of the elements of culture organized by a low-high visibility dimension can be found in Figure 3 below. 


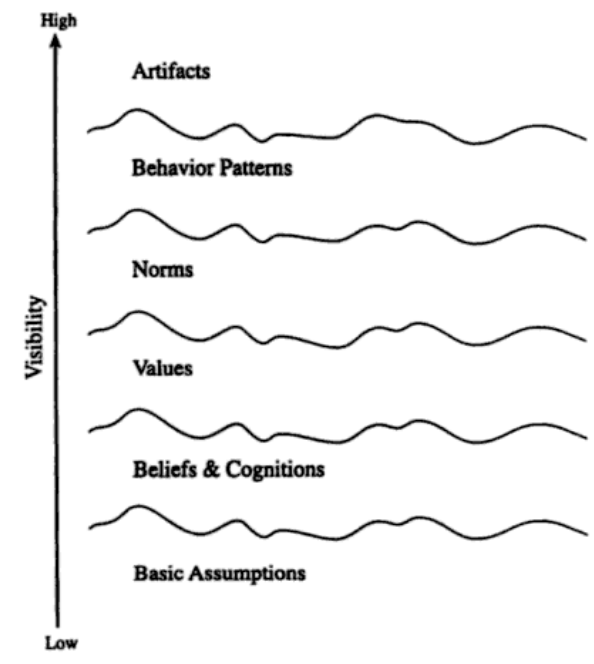

Figure 3: Layers of Organizational Culture. Elements of Organizational Culture arranged in layers, progressing from "low" to "high" visibility. Reproduced from Harrison, 1999, p.260, Figure 10.1.

As portrayed in Figure 3, the majority of definitions and models offered for organizational culture have in common a tendency to treat values, beliefs and assumptions as deeper or more fundamental attributes. Over the past three decades, organizational scholars seeking to advance the investigation of organizational culture have proposed a wide variety of dimensions to help organize research efforts. The following paragraphs describe a brief narrative history of key developments in the field, beginning with Hofstede's (1980) seminal work, Culture's Consequences. The year of this groundbreaking publication marked the end of a period where the concept of culture was thought by many to be confined to the discipline of anthropology (Triandis, 2004). Hofstede focused on four dimensions of culture: 1) individualism vs. collectivism; 2) masculinity vs. femininity; 3) power distance; and 4) uncertainty avoidance (Hofstede, 1980). Hofstede's proposed dimensions quickly became influential throughout the 
social sciences and vast bodies of research were generated through them (Triandis, 2004).

Building on the momentum generated in the early 1980 s, organizational culture research as a field of inquiry experienced major growth in 1985. In this year alone, major developments in the proposed dimensions of organizational culture included: 1) an internal vs. external focus dimension and strong-weak cultural dimension by Arnold and Capella (1985); 2) an idiographic vs. holographic dimension of organizational culture by Albert and Whetten (1985); 3) a people orientation (participative vs. nonparticipative dimension) and environmental dimension (reactive vs. proactive orientation) were argued as critical by Ernest (1985); and 4) eleven other dimensions of culture, including "clarity and direction, organizational reach, integration, top management contact, encouragement of individual initiative, conflict resolution, performance clarity, performance emphasis, action orientation, compensation, and human resource development," were identified in by Gordon in Kilmann (1985) (Cameron \& Quinn, 2011, p. 36).

De Vries and Miller (1986) turned attention to dysfunctional dimensions of culture (including paranoia, avoidance, bureaucracy, and politics), a work which is clearly echoed in Lencioni's (2006) work on the five dysfunctions of a team. Arnold and Capella (1985) proposed the strong vs. weak culture dimension, included in the work of Kotter and Heskett (1992), where they argue that the cultural strength dimension and "congruence" are the most important cultural dimensions). Arnold and Capella (1985) also proposed an internal vs. external focus dimension which has continued to enjoy 
attention in scholarly work. The internal vs. external dimension appears in Quinn's (1988) Competing Values Framework.

Many other dimensions of organizational culture have been proposed, and the list of those yet to be proposed will never end because, as Cameron and Quinn (2011) asserted, organizational culture is a complex, ambiguous, extremely broad concept. Today, the Competing Values Framework is the world's most widely used framework for assessing organizational culture (Cameron \& Quinn, 2011), and is discussed further below.

\subsubsection{The Competing Values Framework.}

While the dimensions and definitions proposed for understanding organizational culture have been diverse and sometimes inconsistent over the last 34 years, Robert Quinn's Competing Values Framework has emerged as a clear leader in terms of most used theoretical model for assessing organizational culture (Cameron \& Quinn, 2011). The Competing Values Framework was first developed by Quinn and Rohrbaugh (1983). They derived the framework from a statistical analysis of 39 indicators for measuring organizational effectiveness (Cameron \& Quinn, 2011). Two major dimensions emerged from their analysis, focus and structure, which became the $X$ and $Y$ axes of the Competing Values Framework (as seen in Figure 1).

The two dimensions of the competing values framework can be further described as flexibility vs. stability in the organizational Structure, and an internal vs. external organizational Focus. As stated previously, the four archetypal cultures in the 
CVF (Clan, Adhocracy, Market, and Hierarchy) are often depicted as quadrants along two dimensions (see Figure 1).

The Clan Archetype, in the upper left quadrant of the CVF, is associated with family-type organization concepts, or where a culture seems "more like extended families than economic entities," and is permeated by "[s]hared values and goals, cohesion, participativeness, individuality, and a sense of 'we-ness'"(Cameron \& Quinn, 2011, p. 46). The success of Japanese firms since World War II has helped send the message to U.S. and Western European firms that "clan cultures can make good business sense"(Cameron \& Quinn, 2011, p. 47).

The Adhocracy Archetype, in the upper right quadrant of the CVF, is associated with entrepreneurial organization concepts, where creativity, adaptability, and innovation are highly valued. Assumptions that underlie the adhocracy culture are that “innovative and pioneering initiatives lead to success, organizations are mainly in the business of developing new products and services and preparing for the future, and the major task of management is to foster entrepreneurship, creativity, and activity on the cutting edge" (Cameron \& Quinn, 2011, p. 49).

The Market Archetype, in the lower right quadrant of the CVF, strives for strong external positioning and control and is associated with competitive organizations that focus on market dominance, bottom-line results, profitability, productivity, transaction costs, secure customer bases, and stretch targets (Cameron \& Quinn, 2011). The basic underlying assumptions of a market culture are summarized by Cameron and Quinn in this way: 
The external environment is hostile rather than benign, consumers are choosy and interested in value, the organization is in the business of increasing its competitive position, and the major task of management is to drive the organization toward productivity, results, and profits... [and] that a clear purpose and aggressive strategy lead to productivity and profitability. (Cameron \& Quinn, 2011, p. 45)

The Hierarchy Archetype, in the lower left quadrant of the CVF, is characterized by a control-oriented, formalized and structured workplace (Cameron \& Quinn, 2011). Clearly structured decision-making authority, rules, accountability, efficiency, impersonality, and smooth, predictable output are highly valued in these organizations. "Large organizations and government agencies are generally dominated by a hierarchy culture, as evidenced by large numbers of standardized procedures, multiple hierarchical levels (for example, Ford has seventeen levels of management), and an emphasis on rule reinforcement" (Cameron \& Quinn, 2011, p. 42).

The instrument used to diagnose organizational culture based on the Core Values Framework is the Organizational Culture Assessment Instrument (OCAI). This OCAI and its application will be discussed in the following section.

\subsubsection{Diagnosing Organizational Culture.}

While there are many proposed frameworks and methods for diagnosing organizational culture, the focus of this research is on extending the diagnosis of organizational culture based on the CVF. The CVF was chosen because it is "the dominant framework in the world for assessing organizational culture" (Cameron \& Quinn, 2011, p. 35). The most current and thorough guide for diagnosing organizational culture based on the CVF is Cameron and Quinn's 2011, $3^{\text {rd }}$ Edition of Diagnosing and 
Changing Organizational Culture: Based on the Competing Values Framework. As such, that work is referenced extensively in this section, which focuses on the use of the OCAI instrument in diagnosing organizational culture.

The OCAI assesses "six key dimensions of organizational culture" based on the CVF (Cameron \& Quinn, 2011, p. 28). The six dimensions of organizational culture assessed through the OCAI are 1) Dominant Characteristics, 2) Organizational Leadership, 3) Management of Employees, 4) Organizational Glue, 5) Strategic Emphases, and 6) Criteria of Success. While these six dimensions are not comprehensive, they describe some fundamental aspects of organizational culture that "have been found to be equally predictive of an organization's culture" as longer assessments (Cameron \& Quinn, 2011, p. 28).

The OCAI (Appendix G contains the full OCAl instrument) has six questions, one question for assessing each of these six key dimensions. Each question has four alternatives, with each alternative corresponding to one cultural archetype found in the CVF (see Figure 4 below for an example). Participants are instructed to rate their organization by dividing 100 points among the four alternatives, allocating points to the extent that each alternative is similar to the organization. 


\begin{tabular}{|c|c|c|c|}
\hline \multicolumn{2}{|c|}{ 1. Dominant Characteristics } & \multirow[t]{2}{*}{ Now } & \multirow[t]{2}{*}{ Preferred } \\
\hline A & $\begin{array}{l}\text { The organization is a very personal place. It is like an } \\
\text { extended family. People seem to share a lot of themselves. }\end{array}$ & & \\
\hline $\mathrm{B}$ & $\begin{array}{l}\text { The organization is a very dynamic entrepreneurial place. } \\
\text { People are willing to stick their necks out and take risks. }\end{array}$ & & \\
\hline $\mathrm{C}$ & $\begin{array}{l}\text { The organization is very results oriented. A major concern is } \\
\text { with getting the job done. People are very competitive and } \\
\text { achievement oriented. }\end{array}$ & & \\
\hline \multirow[t]{2}{*}{ D } & $\begin{array}{l}\text { The organization is a very controlled and structured place. } \\
\text { Formal procedures generally govern what people do. }\end{array}$ & & \\
\hline & Total & & \\
\hline
\end{tabular}

Figure 4: The First Question of the Organizational Culture Assessment Instrument. Source: Cameron/Quinn, Diagnosing and Changing Organizational Culture, (C2000.

Participants are also instructed to complete two passes through all six questions in the OCAl, first assessing the organization as it is "Now" (in the first column provided, followed by assessing how they would prefer to see the organization in five years (in the "Preferred" column provided). See figure 4 above, or Appendix F for the complete OCAI assessment.

For all questions in the OCAI, the $\mathrm{A}$ alternative corresponds to the Clan culture archetype, B corresponds to Adhocracy, C corresponds to Market, and D to Hierarchy. Scoring an individual's OCAl is achieved by computing an average score for each alternative (for example, adding all " $A$ " responses together, and then dividing by the number of questions to assess the organization's overall Clan culture profile). Once average scores are computed for all four alternatives in both columns, the results are usually plotted (example provided in Figure 5 below). 


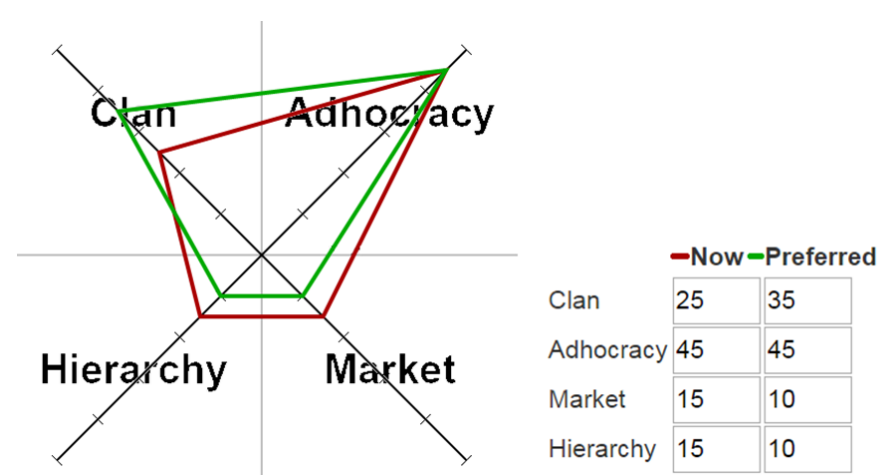

Figure 5: Culture profile example generated from an OCAI assessment. Figure shows an example of the plotted results of a culture assessment based on the results of an Organizational Culture Assessment Instrument. The dominant culture in this example is Adhocracy.

Complete assessment of organizational culture through the OCAI requires that results be plotted in two ways: as an overall culture profile (an aggregate of results from all six questions on the OCAl, as in figure 5) and as a collection of plots for individual items on the OCAI (i.e., the results from one question on the OCAI). Having completed these two sets of plots allows for at least six standard analyses: 1) the type of culture that dominates an organization; 2) discrepancies between current and preferred future culture; 3) the strength of the culture type that dominates an organization; 4) the congruence of the culture profiles generated on different attributes and by different individuals in the organization; 5) a comparison of an organization's culture with average culture profiles; and 6) comparison of data against trends generated by other organizations (approximately 1000) other organizations analyzed in the past 20 plus years of OCAI use (Cameron \& Quinn, 2011). A considerable body of literature exists on how to interpret and utilize results from these standard analyses, most of which is synthesized or cited in Cameron and Quinn (2011).

\subsection{Literature on Organizational Values}




\subsubsection{Importance \& Definition of Values.}

There is a large body of literature touting the importance of values in organizations. Values can be defined as concepts or principles that transcend specific situations and guide the selection or evaluation of behavior and events (Schwartz, 2012). Richard Barrett, a leading values-scholar, made the case in his most recent book that "values-driven organizations are the most successful organizations on the planet," and concludes that "understanding [employee values] is the key to creating a high performance organization" (Barrett, 2013, p. xi).

In their book Built to Last: Successful Habits of Visionary Companies, Collins and Porras made a strong case that the best performing companies have and adhere to a unique set of guiding principles called core values (Collins \& Porras, 1994). Core values are values with more influence on behavior or evaluation than most other values in a system (Pant \& Lachman, 1998), and they have intrinsic importance and meaning to organizational members (Van Rekom et al., 2006). Core values, unique to each firm (Collins \& Porras, 1994; Lencioni, 2002), play a major role in guiding strategic decision making (Marcus, 2004) and aligning corporate strategy and culture. Collins and Porras' work provoked a major shift in focus to the critical role of values to organizational success, and today the majority of companies have crafted and published values statements.

It has also been argued, however, that the majority of values statements today are empty, or what Lencioni (2002) calls "bland, toothless, or just plain dishonest" (p. 5). Such values statements are ineffective, costly, and even destructive. More than just a 
missed opportunity, empty values statements have also been charged with undermining managerial credibility, fostering cynical work environments, dispiriting employees, and alienating customers (Lencioni, 2002). An understanding of how to differentiate between core and other types of values is key to avoiding these concerns and will be discussed in the following section.

\subsubsection{Types of Organizational Values.}

The purpose of the present research was to develop a technique for assessing organizational core values based on the Competing Values Framework. Therefore, understanding the different types of organizational values and how to discriminate between them was necessary. Core values have been described as 1) a unique ${ }^{1}$ and 2) enduring $^{2}$ set of guiding principles ${ }^{3}$ (Collins \& Porras, 1994; Lencioni, 2002), 3) with more influence on behavior or evaluation than most other values in a system (Pant \& Lachman, 1998; Van Rekom et al., 2006), 4) with intrinsic importance and meaning to organizational members (Collins \& Porras, 1994; Van Rekom et al., 2006). While scholars seem to agree that core values are unique, the core values reported in various studies are often somewhat generic, short declarations such as "Systems Perspective,"

\footnotetext{
1 “[Core values] are the source of a company's distinctiveness" (P. M. Lencioni, 2002, p. 6). Barney suggests that, while some aspects of an organizational culture may be perfectly imitated, "the constellation of persistent symbols, beliefs, and values that characterize a firm's culture at least partially reflect the unique early history of the firm, including the pattern-setting influence of company founders" (Barney, 1986, p. 661).

${ }^{2}$ Collins and Porras define core values as "The organization's essential and enduring tenets - a small set of general guiding principles" (Collins \& Porras, 1994, p. 73). They later describe core values as "values so fundamental and deeply held that they will change or be compromised seldom, if ever" (Collins \& Porras, 1994, p. 74).

${ }^{3}$ Taylor et al. elaborate by defining core values as "shared beliefs that guide decisions and behaviors as people conduct day-to-day work and interact with each other. They are a reflection of the culture of the organization" (Taylor, Van Aken, \& Smith-Jackson, 2007, p. 1).
} 
"Focus on Quality," or "Service-Oriented." ${ }^{4}$ In cases where core values appear as somewhat generic phrases, the uniqueness of core values can be elucidated through the use of associated statements, such as "Systems Perspective: We consistently consider what is best for the department as a whole rather than focus areas, labs/centers, or individuals when making decisions and conducting our day-to-day work" (Taylor, Van Aken, \& Smith-Jackson, 2007, p. 2).

Southwest Airlines' offers a strong example of concise, non-generic core values. The published core values at Southwest Airlines are "Warrior Spirit," "Servant's Heart," "Fun-LUVing Attitude," and "Work the Southwest Way." ${ }^{5}$ Because core values should also provide a clear blueprint for employee behavior (i.e., core values are guiding principles), Southwest Airlines also maintains a set of behaviors associated with each core value (see Figure 6 below).

\footnotetext{
${ }^{4}$ These examples are drawn from the 2006 list of core values of the Malcolm Baldrige National Quality Program, as published in (Taylor et al., 2007).

${ }^{5}$ As published on Southwest Airline's official webpage, http://www.southwest.com/html/aboutsouthwest/careers/culture.html , accessed 5/15/2014.
} 


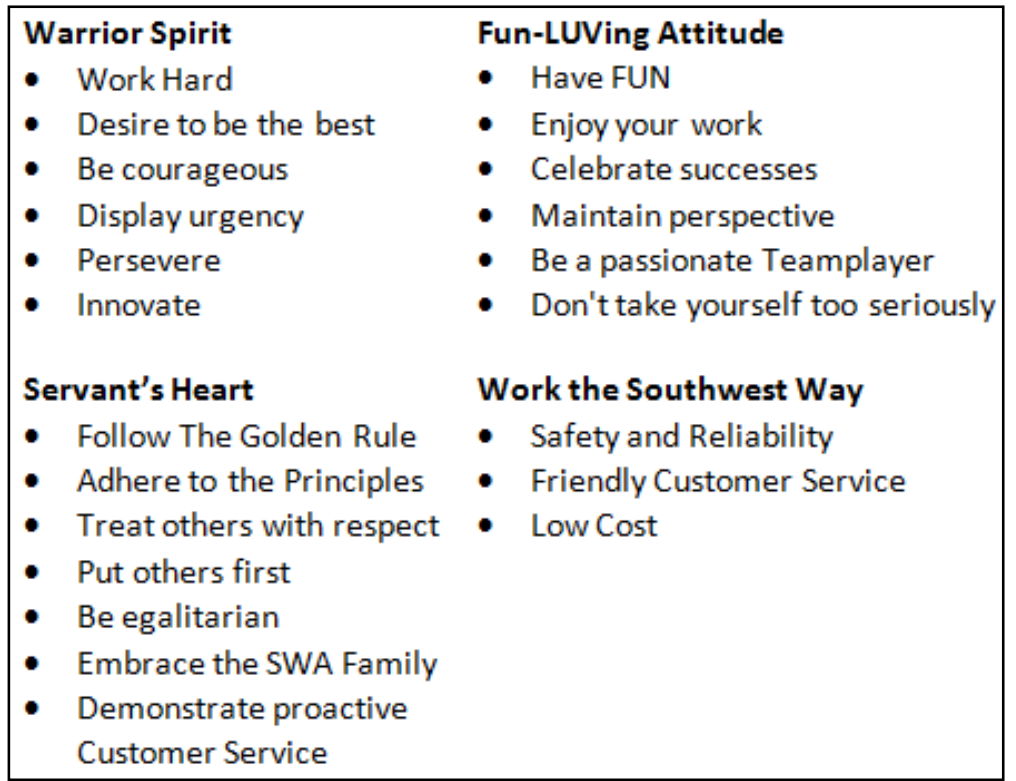

Figure 6: Southwest Airlines Core Values and behaviors associated with each. Southwest Airlines' core values statement is a popular exemplar of high-quality articulation. Source: http://www.southwest.com/html/about-southwest/careers/culture.html

There are several other types of organizational values which can be distinguished from core values. Borne and Jenkins argued that "there are four distinct forms or organizational values - espoused, attributed, shared and aspirational" (Bourne \& Jenkins, 2013, p. 495) ${ }^{6}$. Espoused values are defined as values determined or advocated by the top management team and often stated in corporate documents (Bourne \& Jenkins, 2013). While there may be overlap between core values and espoused values, this is not necessarily the case. Some espoused values can be differentiated from core values if they do not exhibit more influence on behavior or evaluation than most other values in a system, and if they do not have intrinsic importance and meaning to organizational members.

\footnotetext{
${ }^{6}$ It is important to note that Bourne and Jenkins do not discuss core values in this paper, and so the relationship between core values and their four proposed types is unclear.
} 
Attributed values are described as "those that members generally regard as representative of the organization" (Bourne \& Jenkins, 2013, p. 499). Attributed and core values will overlap where they are unique, intrinsically important to organizational members, and more influential than most other values in the system. Attributed values can be distinguished from core values where they are not unique to the organization, important to organizational members, or highly influential values.

Shared values are those shared by organizational members, constituting the organizational value system, measurable in terms of "the proportion of members who share the values" and the intensity of the importance in which it is held relative to other values in the system (Bourne \& Jenkins, 2013, p. 500). To the degree that core values are also shared values, management and organizational change efforts will be easier and more effective (Pant \& Lachman, 1998). Not all shared values are core values, however, as they are not always unique, guiding, more meaningful or influential than other values. Examples of shared values that do not qualify as core may include aspirational values, accidental values, permission-to-play values, or potentially limiting values, which are discussed below.

Aspirational values, those that "members believe ought to be the values of the organization" (Bourne \& Jenkins, 2013, p. 501), have also been defined as "those that a company needs to succeed in the future but currently lacks" (Lencioni, 2002, p. 6). Aspirational values are very influential in organizations because they represent an organizations' response to growing needs or changing circumstances. Aspirational values can be distinguished from core values in that core values are enduring principles 
which do not change in response to short-term circumstance. Aspirational values were included in the Types of Organizational Values Diagram (Appendix L) used in the present research to assist interviewees in distinguishing between core and aspirational values during the second semi-structured interviews of the applied CCVI Technique. The OCAI employed in this research (as part of the applied CCVI Technique) also offers clear insight into aspirational values by allowing the researcher to assess the gap between the current and preferred (future) organizational culture.

Patrick Lencioni (2002), a highly sought after management and leadership expert $^{7}$, has also described core, accidental, and permission-to-play values. Lencioni defined core values as "the deeply ingrained principles that guide all of a company's actions; they serve as its cultural corner stones... They are the source of a company's distinctiveness" (Lencioni, 2002, p. 6), which is compatible with the definition used in this proposed research.

Accidental values are values which, "arise spontaneously without being cultivated by leadership and take hold over time" (Lencioni, 2002, p. 6). Accidental values may also result unintentionally as side effects of structures or processes, such as hiring, firing or incentive systems. Accidental values were included in the Types of Organizational Values Diagram (Appendix L) used in the present research to assist interviewees in distinguishing between core and accidental values.

\footnotetext{
${ }^{7}$ Pat Lencioni has authored 10 books, selling over 4 million copies; is one of the most sought after business speakers in America according to the Wall Street Journal; and founder of the Table Group, a high-profile consulting firm that specializes in organizational culture and leadership. Source: http://www.tablegroup.com/pat/
} 
Lencioni also described Permission-to-play values as values that "simply reflect the minimum behavioral and social standards required of any employee" (Lencioni, 2002, p. 6). Lencioni asserts that permission-to-play values, such as 'integrity' for example, cannot be core values if they do not help distinguish an organization from others. This statement illustrates Lencioni's view of core values as a differentiating feature of organizations, or "the source of a company's distinctiveness" (Lencioni, 2002, p. 6). Permission-to-play values were included in the Types of Organizational Values Diagram (Appendix L), which was used to assist interviewees in distinguishing between core and other types of values.

"Potentially limiting values" were discussed by Richard Barrett as essentially negative values (Barrett, 2013). While examples of positive organizational values may include creativity or teamwork, potentially limiting values may produce issues such as blame, bureaucracy or short-term focus (Barrett, 2013). In the present research the term "potentially limiting values" is used to refer broadly to significant counterproductive values. This use may vary slightly from Barrett's original definition, as he explicitly states that "potentially limiting values arise from the fears and anxieties we have about not being able to meet ... [our] needs" (Barrett, 2013, p. 14). However, to maximize the useful applicability of this term, "potentially limiting values" was used in the present research to refer to any values which are considered negative or potentially limiting, without consideration for the source of those values. Potentially limiting values have been included in the Types of Organizational Values Diagram (Appendix L) used in 
the second round interviews of the CCVI Technique to assist interviewees in distinguishing between core and other types of values.

In summary, in addition to core values, four types of organizational values have central relevance to this research: aspirational values, accidental values, potentially limiting values, and permission-to-play values. These particular four types of relevant organizational values were selected because each contrast clearly with core values and will therefore be useful in the process of classifying top organizational values as either core or otherwise.

\subsubsection{Diagnosing Organizational Core Values.}

The specific aim of the CVF-based Core Value Identification (CCVI) Technique developed in this research is to identify core values based on the Competing Values Framework (CVF). As discussed in section 2.2.3, the Organizational Culture Assessment Instrument (OCAI) is the standard CVF-based tool used to assess organizational culture, which includes cultural values. Well-known, extensively validated, standardized instruments such as the OCAl exist for assessing organizational values (Bellot, 2011), but these approaches "suffer from important drawbacks when establishing the core values of a specific organization" (Van Rekom et al., 2006, p. 176). A key drawback is that standardized instruments typically include lists of generic values, with which "there is no guarantee at all that these lists include the specific core values of the organization" (Van Rekom et al., 2006, p. 177). As previously stated, it has long been known that standardized instruments tend to stress common features that exist across many organizations, but often do not help investigators discover unique patterns, values or 
cultural features within particular organizations or their subcultures (Harrison \& Shirom, 1999).

For this reason, inductive procedures are often used to select the initial set of values to be included in the selection process in site-specific research contexts (Meglino \& Ravlin, 1998). Common methods for qualitative data collection in organizational contexts are interviews, participant observation and document review (Charmaz, 2006), all of which were employed in the present research. Common methods for obtaining an initial set of values from which to select core values are interviews or meetings with management team members to discuss organizational values (Bernstein \& Audley, 1986; Lencioni, 2002). Rekom et al. (2006) propose a two-step method for assessing organizational core values: exploratory interviews with management followed by a survey to assess patterns between values identified in the interviews. Similarly, the present research included interviews with company leaders to both establish the initial set of values and to assist in identifying core values from the list. Follow-up interviews were also used in this research, which serves a purpose similar to the follow-up survey in the Rekom et al. (2006) process.

Participant observation and document review were also employed in this research, in addition to interviews with company leaders. Participant observation is included because "measurement of core values should begin in daily corporate life, by analyzing the concrete behaviour of organization members" (Van Rekom et al., 2006, p. 177). Van Rekom et al. strongly suggest that participant observation is currently lacking in studies of organizational core values, saying, "Values in the context of day-to-day organizational behaviour have not 
received much attention. The striking fact is that none of the methods available is grounded in daily behaviour" (Van Rekom et al., 2006, p. 177). As mentioned, the present research included participant observation of day-to-day organizational behavior because the resulting CCVI Technique is a systems approach for triangulating unique organizational core values, requiring multiple perspectives and various data sources. 


\section{Chapter 3: Research Design \& Methodology}

The research design can be understood as a process of iteratively refining a technique that extends the application of the CVF to assess culture and identify core values at a series of three organizations. The CCVI Technique developed in this research applied grounded theory data analysis techniques to uncover organizational core values in a series of three exploratory case studies of local companies. A case study methodology was used in this research during each iterative application of the developing CCVI Technique for each subject company.

Studies focused on, "culture in a group, a program, or an organization typically espouse some form of case study as a strategy" (Marshall \& Rossman, 2010, p. 93). The qualitative case study methodology is a strategy for executing a detailed analysis of complex phenomena in their context; it therefore entails immersion in the setting being studied (Baxter \& Jack, 2008; Marshall \& Rossman, 2010).

The present research primarily used a qualitative approach for two main reasons. First, qualitative research is appropriate for engaging in exploratory research where the relevant variables have not been identified (Marshall \& Rossman, 2010). This research took place on site at three subject companies in Portland, Oregon. No organizational study had previously been published on the unique culture of these firms, and the desire to uncover their unique organizational values strongly suggested qualitative methods (Marshall \& Rossman, 2010). Second, qualitative research is well suited to capturing the subjective understanding of individual participants (Marshall \& 
Rossman, 2010). This research explored employees' subjective understandings of company values in order to triangulate organizational core values at each subject company. While this research primarily used a qualitative approach, two minor quantitative elements were included. Quantitative elements of this research were: 1) the use of the OCAI instrument as designed; and, 2) the use of ranking sheets on which interviewees rank and weight organizational values according to their perceived impact on organizational performance during applications of the CCVI Technique.

\subsection{Research Questions}

This research had two levels of purpose and therefore employed two levels of research questions. The primary purpose of this research, to develop a technique for assessing unique core values based on the CVF, was addressed by a methodology that included the use of three qualitative case studies to refine the CCVI Technique. Each application of the CCVI Technique included a case study with its own purpose, which was to provide a culture assessment of each subject company which includes organization's unique core values. The overarching methodology for this research is discussed in the following section, and was designed to iteratively address a single primary research question (RQ\#1):

RQ\#1: What is an effective technique for assessing organizational core values based on the CVF?

Addressing RQ\#1 occurred primarily in the iterative Technique Refinement Phase and the Final Analysis steps of this research, resulting in the iteratively refined CCVI Technique for assessing unique core values based on the CVF. Addressing RQ\#1 
resulted in the achievement of the primary purpose of this research, which was to develop a technique for assessing unique core values based on the CVF.

This research also included three qualitative case studies, where each was designed to address the secondary purpose of this research: to provide a culture assessment of each subject company which includes the organization's unique core values. The CCVI Technique being developed in this research was applied in each case study to answer a single applied research question ( $A-R Q \# 1)$, after gathering company data that answers three related sub-questions at each subject company (A-RQ\#1a, A$R Q \# 1 b, \& A-R Q \# 1 c):$

A-RQ\#1: What are the unique core values at each subject company? A-RQ\#1a: What are the values articulated by the leadership team? $A-R Q \# 1 b$ : What values are reflected in the behaviors of the employees? $A-R Q \# 1 c$ : What are the values articulated in the formal policy? Answering A-RQ\#1 at each subject company was the central aim of each case study included in this research project. Investigating A-RQ\#1 at each subject company also provided an opportunity for iteratively testing and refining the CCVI Technique, the development of which was the primary purpose of this research.

Answering A-RQ\#1a resulted in obtaining a description of company leadership's values and understanding of organizational values. This result allowed the researcher to include these understandings in the process of identifying unique organizational core values $(A-R Q \# 1)$, as per the purpose of each case study in this research. 
Investigating $A-R Q \# 1 b$ allowed us to validate leadership's perspectives on current cultural values (in the OCAI assessment) and identify organizational values manifest in group behavior norms for inclusion in the process of identifying unique organizational core values (A-RQ\#1).

Completing A-RQ\#1c resulted in obtaining a complete collection of company policies and an understanding of the values articulated in them for inclusion in the process of identifying unique organizational core values (A-RQ\#1).

\subsection{Overall Approach and Rationale}

This research employs two layers of methodology which reflect the two layers of purpose and research questions. The first layer is called the meta-methodology, the purpose of which was to develop a technique for assessing unique core values based on the Competing Values Framework (CVF). The meta-methodology for this research can be seen in Figure 7, which illustrates the relationship between the individual case studies and overall process.

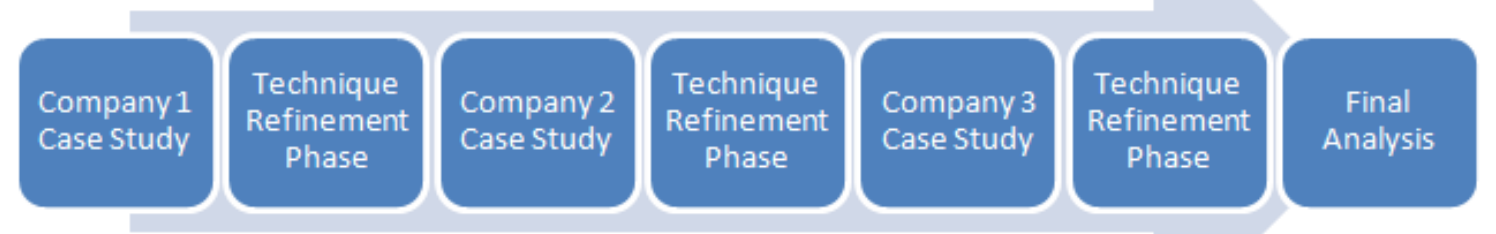

Figure 7: The Meta-Methodology for this research. Figure illustrates the relationship between the individual case studies and overall process. 
Applied methodology in this research. The second methodological layer is the qualitative case study methodology that was applied at each of the three subject companies, where the purpose of each was to identify the organizational core values. The qualitative case study methodology, embedded within the meta-methodology, included semi-structured, in-depth interviews, participant observation and review of documents for data collection and grounded theory methods for qualitative analysis. This was appropriate because a central requirement of this research is to gain an understanding of the three subject company's cultural values, an unexplored phenomenon best studied in its real-life context. The qualitative case study methodology emphasizes detailed analysis of complex phenomena in their context (Baxter \& Jack, 2008). A case study design also allowed for multiple data collection methods that allowed the researcher to answer each research question in turn.

The semi-structured interviews provided the information necessary to address ARQ\#1a ("What are the values articulated by the leadership team?") at each subject company. The participant observation element of the case study design provided the information to address A-RQ\#1b ("What values are reflected in the behaviors of the employees?") at each subject company. A review of company documents provided the information necessary to address A-RQ\#1c ("What are the values articulated in the formal policy?") at each subject company. RQ\#1, ("What are the unique core values at each subject company?") was answered by applying the grounded theory qualitative data analysis process. 
Eight steps were followed at each subject company. These eight steps are described in general in this section, and also comprise the primary result of this research, the CCVI Technique. Therefore, the eight steps outlined below are described in detail in Chapter 4.1 through 4.8, and further discussed in Chapter 6.1 through 6.8. Several of the appendices in the present research are also documents integral to the applied research process and follow the logical order of their usefulness in the research process, beginning with Company Recruitment Letters (Appendix A), followed by Informed Consent Requests for the owner of participating companies (Appendix B), and Interviewee Informed Consent Requests (Appendix C).

Step 1 was conducting initial semi-structured interviews with four to five leaders at each subject company. The questions to start off the first interview included topics about culture description, cultural fit, personal and professional values, and what is valued within the company. The OCAI was administered after initial open-ended inquiry was completed to prevent the implicit CVF framework of the OCAI from biasing initial data. A complete step-by-step overview of initial interviews can be found in Appendix D: Initial Interview Protocol, including interview start up questions. A basic demographic survey included in this step can be found in Appendix E; the Initial Interview Worksheet can be found in Appendix F; and the OCAl and associated scoring sheet can be found in Appendix G. 
Step 2 was conducting participant observation at each subject company. The researcher immersed in the culture as a participant observer at each subject company for approximately one week $^{8}$.

The purpose of participant observation in this research was to validate interviewed leaders' perspectives on current company culture and identify current cultural values implicit in employee behavior that may be included in the discussions with interviewees in Step 5. A Participant Observation Field Guide was constructed for this research, and can be found in Appendix $\mathrm{H}$. The purpose of the Participant Observation Field Guide was to assist the researcher in focused data collection during participant observation. It contains a reiteration of the purpose of participant observation, the extent of disclosure of study purpose to participants and a checklist of steps for completing participant observation.

Step 3 was a review of company documents. The goal of document review in this research was to identify and include formal company policies and publications in the process of identifying core values. The process at each subject company began with researcher and the leadership team identifying and collecting all published materials which may articulate company values. Likely candidates for inclusion were preexisting values statements, employee handbooks, employee evaluations, operating procedures, HR manuals, suggestion cards, surveys, or any other materials that articulate company values. Each leadership team then provided the researcher with this set of documents,

\footnotetext{
${ }^{8}$ Depending on the size and complexity of a company being studied, more or less time may be required to achieve "data saturation," the point at which no new relevant information emerges during data collection.
} 
which were then reviewed for articulations of company values or desired employee behaviors.

The three target companies included in this study were small ( $<55$ employees), privately owned firms with a limited degree of formal documentation. Reflection on the available documentation at these small companies revealed that it was more likely to be out of touch with current cultural reality than are the other two forms of primary data collection (interviews and participant observation). In response to this realization, the bulk of the research focus when drafting Core Values was placed on data from interviews and on field notes from observation.

Step 4 was the initial analysis of all data collected in Steps 1 through 3, including OCAI results. Qualitative analysis began with initial open coding of all collected data. Thematic content analysis was then used to move from coding to identifying themes and patterns of themes in the data. As preliminary themes and concepts began to emerge, the theoretical sampling phase began - a targeted data gathering step in grounded theory - where more interviews were conducted to assess whether or not the themes and perspectives of the participants have been understood, to test some preliminary explanations or theories, and to achieve data saturation. The main outputs of this initial analysis were:

(a) completed and analyzed OCAls from all interviewees, the results of which were utilized in the two OCAI results forms used in Step 5;

(b) transcribed and coded initial interviews from all interviewees, the results of which appeared in the Core Values Identification Form used in Step 5;

(c) complete and coded field notes from participant observation, the results of which appeared in the Core Values Identification Form used in Step 5; 
(d) a coded collection of published material at each subject company which articulate company values, the results of which appeared in the Core Values Identification Form used in Step 5; and,

(e) an initial codebook, containing the collective codes, concepts, themes and patterns in the data, which was later used in Step 6 for final analysis.

Step 5 entailed conducting second semi-structured interviews with the same leaders at each company as were interviewed in Step 1. Complete step-by-step instructions can be found in Appendix I: Second Interview Protocol. To establish an advanced starting point for deeper inquiry, the Step 5 semi-structured interviews began with discussion of OCAI results and the Competing Values Framework. The Overall Culture Assessment form Template (see Appendix J) and Itemized Culture Assessment Template (see Appendix K) were created to facilitate the discussion of OCAI results and Core Values Framework in this step. A worksheet describing various types of organizational values was also created for this step and included in Appendix L.

Next, all tentative core values resulting from coded data were presented and interviewees were asked to rank, weight, and discuss top tentative organizational values. A Core Values Identification Form (see Appendix M) was created to facilitate interviewees' ability to choose, prioritize and differentiate core values from other types of values. When presented to interviewees, the Core Values Identification Form contained a list of all important values resulting from initial interviews, observations, and document review at the subject company. The prototype framework for differentiating core values from other types of values that appears on the Core Values Identification Form was constructed by the researcher, based on initial literature review. 
This framework was iteratively enhanced during this research through a combination of the applied thematic content analysis step (Step 4) and reflection during the technique refinement phases of this research process.

Interviewees were then asked to describe the type of each their chosen, prioritized values, differentiating between core and other types of values. Finally, interviewees were asked to assign expected behaviors to each of their chosen, prioritized values. A Value-Behavior Association Form (see Appendix N) was created to facilitate interviewees' ability to assign specific behaviors to the values they ranked as most important. Interviewees were asked to assign specific behaviors to values because core values should provide a blueprint for employee behavior (Lencioni, 2002).

Step 6 involved final coding and analysis of the new collected data from Step 5 using the selective coding method. This was followed by a process called theoretical memoing, which is the iterative, written conceptualizing and theorization of the relationships between codes, and the creation and naming of concepts and categories (B. G. Glaser, 1998). Through a process known as sorting, the many emergent codes, concepts and categories were also pieced together in this step. This process led to the emergence of a descriptive theory of the organizational culture and core values at each subject company. The results of Step 6 were then assessed in Step 7.

Step 7 was conducting final interviews with the same company leaders from Steps 1 and 5 to assess whether or not the researcher has understood the themes and perspectives of the participants. This is a process called member checking, which was used to achieve testimonial validity (Carter, Bryant-Lukosius, DiCenso, Blythe, \& Neville, 
2014). In addition to member checking, final interviews were also used to test some preliminary explanations or theories that may appear in the final presentation and to ensure data saturation has been achieved.

Step 8 was the final presentation of a statement of the identified core values at each subject company, and obtaining acceptance of project deliverables by company owners. "Company owners" refers to the person at each company who granted the researcher access to the organization. For all companies included in this research, this person was the owner.

At the end of each case study a Technique Refinement Phase was conducted (see Figure 7). During this phase the researcher engaged in reflective inquiry, peer debriefing, and asked follow-up questions to client-participants. In this research reflective inquiry included reflecting on the researcher's experience in each case study and identifying opportunities for improvement. Peer debriefing was defined in this work as discussing the application of the proposed technique at each organization with committee members and peers in the academic community. Follow-up questions were asked of client-participants after the Final Presentation of results at each case study, either via email or interview, to assess and improve the technique. Follow-up questions included, "How helpful has this been? Will you be able to inform policy and/or practice with these results? How effective was this technique in achieving our purpose (culture assessment and identifying core values)? What was great? What would you suggest I do differently?"

\subsection{Site and Participant Selection}




\subsubsection{Site Selection.}

Research for each of the three case studies in this project was conducted at the site of operation for each of the three subject companies in Portland, Oregon. These sites were chosen because the purpose of each case study in this research is site- and participant-specific to each subject company. As noted, the primary purpose of this research project was to develop a technique that extends the application of the Competing Values Framework (CVF) to identify unique organizational core values, and the purpose of each case study the three subject companies was to identify organizational core values while iteratively developing the proposed technique.

In particular, A-RQ\#1a ("What are the values articulated by the leadership team?") required in-depth interviews, which are best done in person. On-site participant observation was also required to address A-RQ\#b ("What values are reflected in the behaviors of the employees?"). Therefore, this research was best conducted at the site of operations of the subject companies in Portland, Oregon. In addition, gaining access to these sites was not an obstacle for this research.

\subsubsection{Participant Selection.}

The five criteria for screening participant companies were that companies must: a) have fewer than 55 employees (for reasons explained below); b) have approximately four leaders; c) be intentional about company culture and values; d) have been in business at least five years; and, e) be willing to allow the researcher access to their operations and personnel for interviews, document review and participant observation 
described in this research. These requirements are not industry specific because the CCVI Technique developed in this research is not industry specific.

All on-site employees (over age 18) were able to be included in participant observation because subject companies have fewer than 55 total employees. Because this research is participant-specific, this broad approach was taken to observe the full variety of sub-groups in the organization to facilitate data saturation and the discovery of cultural values (for inclusion as potential core values in discussions with company leaders in Step 5 of the case study process). To address A-RQ\#1a ("What are the values articulated by the leadership team?"), executives or owners of each subject company were asked to identify the members of the leadership team, and all identified and accessible persons were interviewed. Companies recruited for participation in this project had four to five leaders, which included company executives, owners, controllers, top management, and influential employees. To address A-RQ\#1b (“What values are reflected in the behaviors of the employees?"), approximately one week (40 hours $)^{9}$ of participant observation was completed at each subject company.

As an ethical assurance measure, companies were also selected based on intentionality about their values and culture because these companies were in a position to benefit the most from the results of this research. This was done in order to maximize reciprocity in the relationship, offsetting the burden that this research placed on the workplaces of participant organizations.

\footnotetext{
${ }^{9}$ Depending on the size and complexity of each subject company, more or less time may be required to achieve data saturation.
} 


\subsection{Data Collection Methods}

The four data collection methods used in this research were in-depth interviews, the Organizational Culture Assessment Instrument (OCAI), review of documents, and participant observation. In-depth semi-structured interviews were used for A-RQ\#1a (“What are the values articulated by the leadership team?") at each subject company because this is the most effective method for gathering participants' subjective descriptions of values. The OCAI was also administered during in-depth interviews to provide a baseline for later discussing results within the Competing Values Framework. A-RQ\#1b (What values are reflected in the behaviors of the employees?") at each subject company required perception of worker behavior in their natural, complex cultural context, which can only be effectively gathered through participant observation. A-RQ\#1c ("What are the values articulated in the formal policy?") at each subject company was thoroughly addressed through document review. The essential applied research question, $A-R Q \# 1$ (“What are the unique core values at each subject company?") was addressed in the data analysis phase and relied on the data collected for all three of the subordinate applied research questions (A-RQ\#1a through $A-R Q \# 1 c)$.

The primary research question, RQ\#1 ("What is an effective technique for assessing organizational core values?") was addressed primarily in the iterative Technique Refinement Phase ${ }^{10}$ and the Final Analysis step of this research, and was

\footnotetext{
${ }^{10}$ Technique refinement was able to occur during all stages of the research because the researcher constantly engaged in reflective inquiry.
} 
addressed through reflective inquiry ${ }^{11}$, peer debriefing ${ }^{12}$, and asking follow-up questions to client-participants ${ }^{13}$.

\subsubsection{Theoretical Sampling and Saturation.}

The purpose of each case study involved in this research was to individually identify the unique core values at each subject company. Unlike research undertaken to identify commonalities in processes that transcend corporate bounds, the present research sought to identify unique core values that could only be discovered within the specific context of each subject company. Because the core values at subject companies are unique, saturation of data was required within each subject company independent of other companies. Therefore, the number of companies studied had no impact on data saturation within each case study in this research.

The two practical aspects of this research impacted by number of companies studied were the amount of work to be done by the researcher and the number of opportunities the researcher had to iteratively refine and develop the proposed technique for identifying unique organizational core values.

\subsection{Data Analysis}

Grounded theory (GT) qualitative data analysis methods were employed in each of the three case studies within this research project. Grounded theory methods are an iterative process structured to allow theory to emerge from themes, topics and insights

\footnotetext{
${ }^{11}$ Reflecting on the researcher's experience in each case study and identifying opportunities for improvement.

12 Discussing the application of the proposed technique at each organization with committee members and peers in the academic community.

${ }^{13}$ Asking exit questions, either via email or interview, to assess and improve the technique.
} 
from collected data. Analysis began with initial open coding of all collected data. All codes resulting from initial open coding were then recorded in electronic spreadsheet documents, including codes, the source data of each code, and elemental memos ${ }^{14}$. The electronic spreadsheet documents will hereafter be referred to as the codebooks. The codebooks were initially organized under emergent categories or the preliminary categories described in the following section.

Thematic content analysis was then used to move from coding to identifying themes and patterns of themes in the data. This is a focused coding phase where the researcher began by identifying code families and themes amongst code families. This process led to the identification of concepts and categories that further organized the codebooks at the more macro levels. The researcher also constructed tables that trace concepts and categories related to each core value back to original source data in Appendix $\mathrm{O}$, in order to assure transparency regarding the rationale underlying the various code families, concepts and categories. Justification for why decisions were made to group codes in a certain ways was later established primarily through validation and verification of research results by participants. As preliminary themes and concepts began to emerge, the theoretical sampling phase began - a targeted data gathering step - where more interviews were conducted to assess whether or not the themes and perspectives of the participants were understood, to test some preliminary explanations or theories, and to achieve data saturation. This new collected data was then coded and analyzed using the selective coding method. Selective coding progressed much more

\footnotetext{
${ }^{14}$ Elemental memos are brief notes describing ideas captured by or relating to codes.
} 
quickly than initial open coding because the researcher had a sharpened sense of the important themes in the data. In selective coding, the researcher searched for further clarification or detail regarding previously coded data and any new information. When no new important data was discovered, data saturation was achieved.

The next technical step was Theoretical Memoing, an iterative, written conceptualizing and theorization of the relationships between codes, and the creation and naming of concepts and categories. In this phase the researcher pieced the fragmented data together, working to understand how the various codes, concepts, categories and themes all roll up into a cohesive whole. During this theorization process the researcher relied primarily on the specific feedback from interviewees on how to organize the various pieces and justify higher level decisions. This focused, reflective process led to the emergence of higher quality concepts and categories from data that culminated, in the next step, in a descriptive theory of the unique core values at each subject company. Sorting was the final step in the GT process before formally writing up the theory and report, where the researcher took the various codes, concepts and categories that emerged from the data and pieced them together into a cohesive whole, driven by specific feedback from participants on the relationships between elements. The resulting theories of organizational core values at each subject company are described in detail in Chapter 5.

\subsection{How Trustworthiness of Data Was Maintained}

For this research project, the focus of trustworthiness was on three criteria: validity, usefulness and transferability. Validity required that the results of each case 
study are believable from the perspective of fellow researchers and participants in the research (Guba \& Lincoln, 1985). The four methods used for achieving validity were data triangulation from multiple sources ${ }^{15}$, prolonged engagement with the research setting (approximately 40 hours of participant observation at each research site), peer debriefing (particularly discussion of emergent trends and findings with critical peers), and member checking (sharing appropriate data and interpretations with study participants to ensure accuracy). All of these are known procedures for ensuring validity in qualitative case studies (Morse, Barrett, Mayan, Olson, \& Spiers, 2008).

Second, the criterion of usefulness is defined in this research as the degree to which the results are deemed able to inform policy and/or practice by client-participants (Marshall \& Rossman, 2010). Usefulness was ensured through diligence in data collection and analysis, careful study of current literature and asking follow-up questions about the usefulness of results after final presentations.

Finally, the criterion of transferability denotes the ease with which a reader of the final research report may be able to transfer or apply any results of this work to other contexts or settings (Lincoln \& Gubba, 1985). This was achieved through meticulously described details about the research process in particular, thereby allowing maximum transparency for scholars to consider the usefulness of the CCVI Technique in other settings. Case study results are not intended to be transferable, as the cultures, core values, and opportunities for improvement at different organizations should be

\footnotetext{
15 "By triangulating data from multiple sources, investigators considerably enhance the credibility and validity of their inferences" (Harrison \& Shirom, 1999, p. 289).
} 
tailored to each. Case study results are still described in detail in this research to allow other researchers to decide the degree of transferability between this research and other similar settings.

\subsection{Ethical Assurance Measures}

To maintain ethical standards in this research, consent was obtained from company owners (for access to their company and permission to do observations), and from potential interview subjects. The consent form for company owners (see Appendix B) contains a clause that allows participants to withdraw from the study at any time without penalty. When interview subject (all adult subjects, persons 18 years of age and over) agreed to participate in this study, they read and signed an informed consent form that provided further detail about the study and topics to be covered in interviews. This form (see Appendix C) also contains a clause that allows participants to withdraw from the study at any time without penalty. Participants were also allowed to request that any and all portions of the audio recorded interviews be deleted or destroyed at any time. Any use of the data beyond the original intended purpose is subject to written and signed approval from the participants before such use.

To provide the confidentiality described in the informed consent forms, participant names were only used on the consent forms, and all data (recordings, notes, transcriptions and coded data) were kept in the secure location, and will remain in a locked cabinet for three years thereafter. Digital data is stored on a password-protected drive. During data analysis, each participant was given a discrete pseudonym. One list of participant's real names and the pseudonyms given them is kept in the PSU office and 
secured on the PSU password-protected network drive. In addition to confidentiality, this ensures the protection of the data. In order to maintain confidentiality, all discussion of results carefully protected the identities of the participants. The names of individual participants or companies were never used in the writing of papers or communicating to others about this study. Alias names, general titles, and descriptions of company size and industry were used instead.

The researcher also acknowledges that during participant observation, nonexecutive employees involved were informed that observation was taking place and that research was being conducted. Written consent was not required from these employees for observation, nor were they required to be informed of the full nature of this investigation provided that data is not collected through interaction with these subjects. Interview questions, for example, would qualify as interaction and require consent. Observation does not. Permission to conduct participant observation was obtained from the owners of participant companies. Given the vulnerability of nonexecutive employees in participant observation, findings were also reported confidentially and the investigators refrained from reporting any verbatim data which might compromise confidentiality.

During this research there was a change in process where the application of the OCAI was broadened to include all employees at Company C. In this event, where interaction with observed employees became a desired feature of the research, the Human Subjects office was notified of the change in intent, and an informed consent form was approved and provided to these employees, including full disclosure of the 
nature of the research.

Finally, the researcher acknowledges that this research represented a burden on the workplaces and employees of participant organizations. Facilitation of data collection required the time and attention of company employees, and no direct compensation was given to participants. However, this research resulted in providing participant companies with useful information to improve organizational culture or performance in the future. In order to maximize reciprocity in the relationship, the researcher explicitly selected companies who were intentional about their values and culture for participation in this research. Companies intentional about their values and culture were in a position to benefit the most from the results of this research.

In summary, informed consent, confidentiality, and reciprocity in the relationship between subject companies and this research are the most important ethical questions addressed in this work. Informed consent was obtained for all participants, confidentiality was provided for all participants, and the researcher selected companies for participation who were the most likely to benefit from the results of this research.

\subsection{Researcher Biography}

"Although scholars may don a cloak of objectivity, research and writing are inherently ideological activities" (Charmaz, 2006, p. 163). This acknowledgement is echoed by Strauss and Corbin (2007) who write, "Researchers bring to the inquiry a considerable background in professional and disciplinary literature" (p. 35). The researcher also acknowledges that theory does not emerge from data in some perfectly 
replicable way, but is rather negotiated or "constructed" by researchers. It is because of this fact that a brief biography is here recorded, describing the more relevant features of the researcher's ideological and academic grounding, and measures for maintaining the integrity of the research.

The researcher has a background in systems science, and having completed all coursework required for completion of a PhD in Systems Science, acknowledges a sensitivity to systems concepts which may have naturally influenced data collection and analysis. To ensure systems concepts were not unduly influential into the process, member-checking was used in the final interviews. Member-checking is a process where participants were asked to verify the completeness and correctness of the collected data and analysis.

The researcher also acknowledges a preference for working in company environments characterized by the "clan" archetype within the Competing Values Framework. The researcher is aware that this preference may have a sensitizing effect during data collection, where added emphasis may be placed on clan-aspects of subject organizations. The two major controls in place to address this in the present research were completion of the OCAI by company leaders and member-checking. The OCAI completed by company leaders (approximately four per company) provided multiple assessments of the cultural archetype, without any input from the researcher, for triangulating the archetypal base values at each subject company. Member-checking in the final interviews was also used, where participants were asked to verify the completeness and correctness of the collected data. 


\section{Chapter 4: Primary Research Results: The CCVI Technique}

Introduction to Primary Results: The CCVI Technique. The primary purpose of this research project was to develop a technique that extends the application of the Competing Values Framework (CVF) to identify unique organizational core values, addressing RQ\#1 (What is an effective technique for assessing organizational core values based on the CVF?). This chapter presents and describes this primary result, the CCVI Technique (CVF-based Core Values Identification). The eight steps of the CCVI Technique are shown in Figure 8, below.

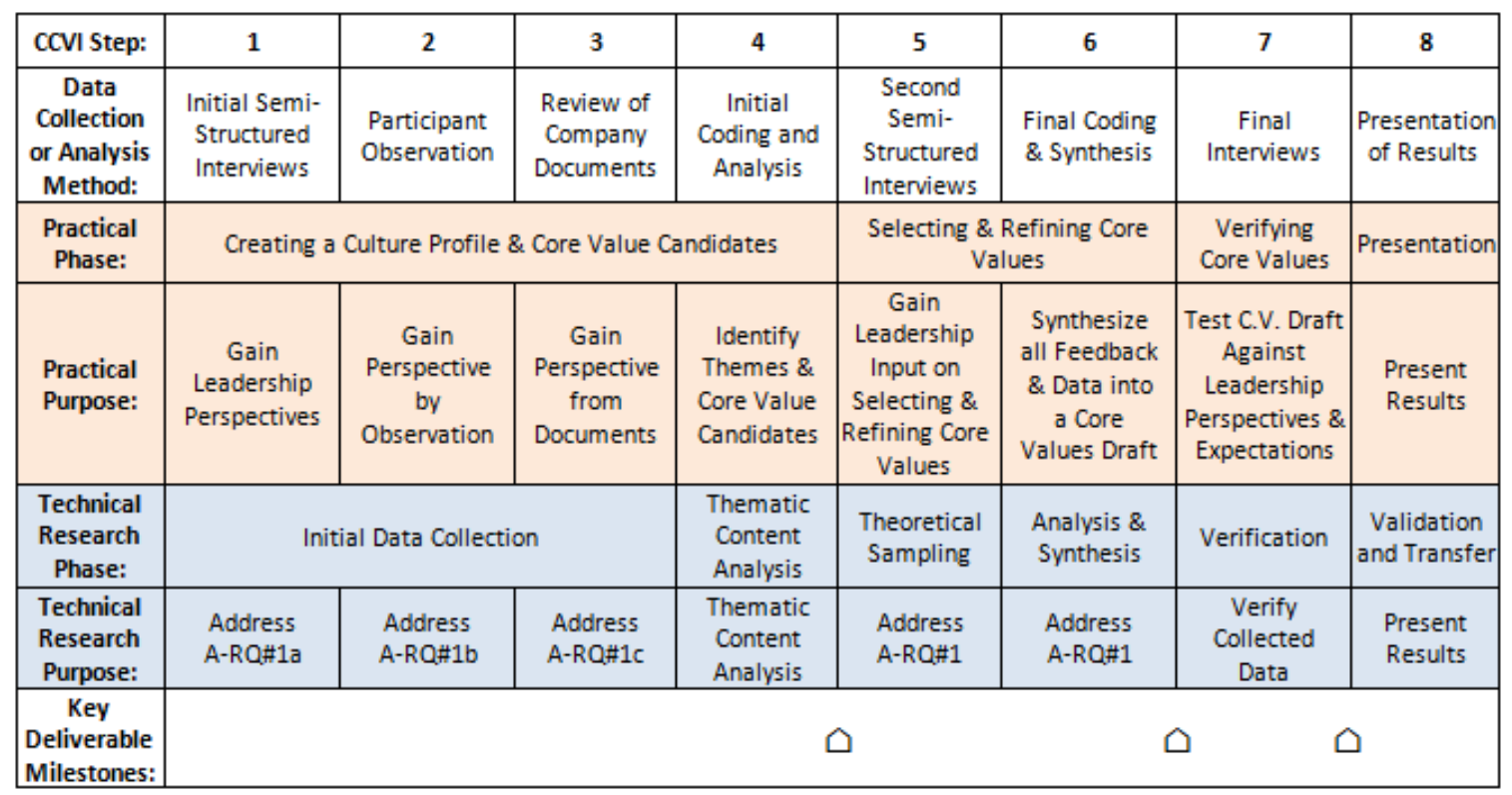

Figure 8: The CCVI Technique Overview (CVF-based Core Values Identification). Figure includes the technique rendered in steps and phases, in language geared toward practitioners (shaded red) and academics (shaded blue), and highlights three milestone deliverables required for successful completion of the CCVI Technique.

The CCVI Technique involves three distinct practitioner phases marked by key deliverables, where the practitioner must produce a specific set of outputs before 
advancing to the next step. The three phases are: (1) Creating a Culture Profile \& a set of Core Value Candidates, (2) Selecting \& Refining of Core Values, and (3) Verification \& Validation of Core Values.

These three phases are overviewed in this introduction from a practitioner perspective (in order to maximize readability for practitioners interested in learning the technique) and then each of the seven CCVI process steps are described in detail in Chapter 4.1 through 4.8, respectively. Additional targeted discussions for each step were also written to enrich the understanding of prospective practitioners, which can be found in Chapter 6.1 through 6.8, respectively. Prior to beginning the process, informed consent from the company owner, CEO or primary decision maker to conduct research must be obtained, participating leaders must be identified, and the length/scope of participant observation agreed upon.

Phase 1 Overview: Creating a Culture Profile \& Core Value Candidates. The first practical phase of the CCVI process is creating a Culture Profile and a set of Core Value Candidates to be used in the second round of interviews with company leaders, all of which play a critical role in drafting core values later in the process. Phase 1 includes the first four steps of the CCVI process, which are initial interviews with company leaders (Step 1), participant observation at the company (Step 2), a review of company documents (Step 3), analyzing the data from those three steps (together called "Initial Data Collection") and preparing it for use in the second round of semi-structured interviews with company leaders (Step 4). Analyzing and preparing the data for the second round of interviews is labor-intensive and marks the first major milestone in the 
CCVI process. The major deliverables from Phase 1 are described with CCVI Step 4 (see Chapter 4.4).

Phase 2 Overview: Selecting and Refining Core Values. The second major phase of the CCVI Technique from a practitioner standpoint is focused on constructing a draft of the Core Values at the target company (the second key deliverable milestone in the CCVI Technique) that will be used in the final round of interviews. There are two steps involved in this process: interviews and data synthesis. The second round of semistructured interviews (Step 5) is when all deliverables from the previous phase (culture assessments, core value candidates, value-behavior association worksheets) are used to guide a dialogue that leads to gaining precise leadership perspective on what the organizational core values are at the target company and to clarify exactly how they should be described. The next step is generating a concise, carefully structured, descriptive statement of core values at the target company. This step, called Data Synthesis (Step 6), can also be understood as constructing a unique and cohesive description of core values by synthesizing all feedback and data. These steps are described in detail in the following sections.

Phase 3 Overview: Verification \& Validation of Core Values. The final practical phase of the CCVI process is testing the Core Values statement created in the previous step against the perspective and expectation of company leadership. This Phase includes the final two steps of the CCVI process, which are the final round of semistructured interviews (Step 7) with company leaders and presentation of results (Step 8). The only goal of this interview is member-checking, and no protocol or formal 
interview questions are necessary. Final presentation of results to company leadership marks the end of the CCVI core values assessment technique, and is described briefly due to its importance in the process of guiding organizational change.

4.1 CCVI Step 1: Initial Interviews. Conducting Semi-Structured Interviews in order to gain leadership perspectives on company culture and values.

The first step in the CCVI process is conducting a one-on-one semi-structured interview with each of the participating company leaders (typically four or five leaders). The Initial Interview Protocol (Appendix D) includes a list of materials to bring, interview questions, and the base script for these semi-structured interviews. The essential flowchart for Initial Interviews can be seen in Figure 9, below. The length of initial interviews can range from 45 to 60 minutes, depending on the brevity or nature of participants. 


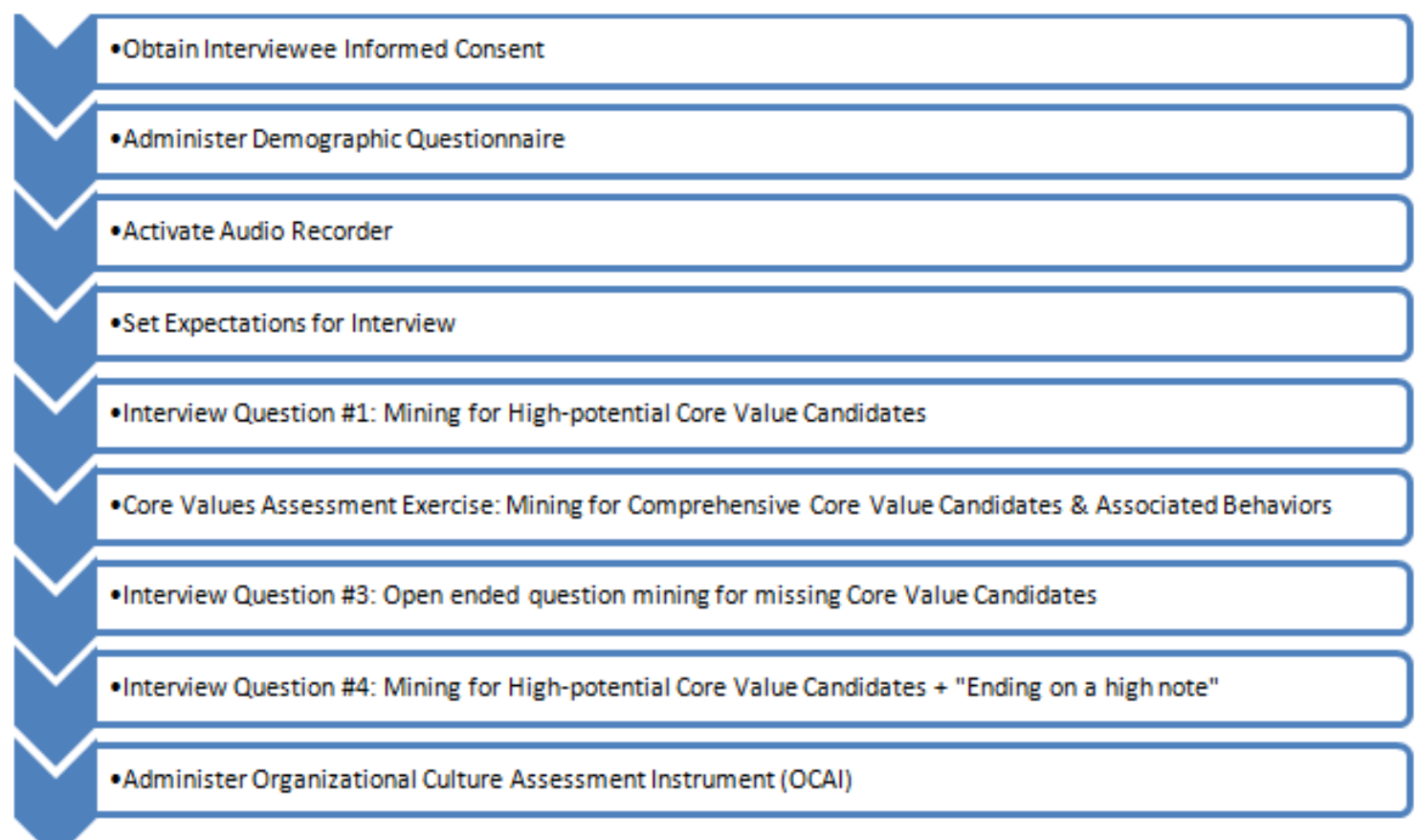

Figure 9: Flowchart for CCVI Technique Step 1: Initial Interview Protocol. See Appendix D for formal protocol. Note that Question \#2 is the set of probes that constitute the Core Values Assessment Exercise shown in this flowchart, as shown in the formal protocol.

Obtaining Interviewee Informed Consent. Two copies of the Interviewee Informed Consent Request should be given to interview participants: one for them to read and sign, and the second for them to keep for their records. Per the Initial Interview Protocol (Appendix D), the Informed Consent Letter should be orally reviewed with participants to ensure they grasp the highlights, including the research objectives, how confidentiality will be maintained, and the scope of their expected participation (three interviews). Collect a signed and dated copy of the Interviewee Informed Consent Request before proceeding.

Administering Demographic Questionnaire. The brief Demographic

Questionnaire used in this research (Appendix E) is intended to collect basic information 
generally required as due diligence in academic research. Administer and collect the questionnaire before proceeding.

Activate Recorder. Request permission from each interviewee to record each interview, as per the Initial Interview Protocol (Appendix D). Recording the interview is critical to the CCVI Technique because coding and analyzing interview transcripts play a key role in identifying themes and core value candidates, and crafting core values in the shared-language of participants. Proceed once permission to record is verbally obtained.

Set Expectations for Interview. Verbally recount the expected timeframe of the interview, the key objectives, and a basic overview of what the participant should expect, as per the Initial Interview Protocol. The essential script used for this research was:

This isn't going to take more than an hour, and if you want to decline any questions that's totally all right. Again, my ultimate objective here is to understand how you, a leader at this company, understand its culture and values. And my goal is also for you to come away refreshed and energized by this conversation. I have about four questions for you, and then a brief assessment for the end. Is that all right?

Upon confirmation, proceed to the next step.

Interview Question \#1: Mining for High-potential Core Value Candidates. The first interview question is, "Tell me, what is one piece of advice you would give to a new employee or applicant about how to thrive at this company?" This question is designed to encourage interviewees to reflect on what the company values in a simple, concrete way. Follow any response or discussion of Question 1 with the probe: "Is there any 
other advice you would give to that new employee or applicant about how to thrive here?" and repeat the process until the interviewee or your professional judgment conclude that the appropriate level of data has been extracted from Question 1. This process of mining Question 1 and probing for other high-priority advice yields a high quality set of data and language for crafting the written Core Values at the end of the CCVI Technique. See Chapter 6.1 for more discussion regarding the important features and effective handling of this interview question.

Core Values Assessment Exercise: Mining for Comprehensive Core Value Candidates \& Associated Behaviors. Following Interview Question 1, ask the interviewee "Can we do a core value assessment exercise?" to which the invariable answer is yes. This exercise acts as the second interview question in the stated four questions described when expectations for the interview are set. Provide the interviewee with the Initial Interview Worksheet (Appendix F). Verbally describe, "there are three groups of employees on the worksheet: Star Employees, Not-so-Hot Employees, and the Leadership Team. We are going to discuss each of these in turn, starting with the Star Employees." Then proceed with probes relating to each employee group and mine appropriately for clarifications and detail. The probes for each employee groups are:

a. Tell me about the best employees or coworkers you've ever had here? No names, but what are some of the VALUES OR BEHAVIORS of people, past or present, who fit really well with the culture - the people who represent the best of the organization? 
b. How about some of the VALUES OR BEHAVIORS of "not-so-hot" employees the people who haven't fit well with the culture. No names, but what are some of qualities of the people who haven't fit well with the culture?

c. Can you tell me about the leadership team? No names, but what are the VALUES OR BEHAVIORS the leadership team that really shape the culture?

The goal is to have the interviewee describe values and behaviors of each employee group verbally, while also bulleting their responses on the worksheet. Some interviewees who prefer speaking require minor prompting to write their responses, while others prefer writing and require prompting to discuss. Ask clarifying questions and mine appropriately for clarity around responses. The section with highest potential value for data mining in this exercise is the star employees section. This is because the values and behaviors shared amongst the star employees - or "most highly valued" employees - are synonymous with the values and behaviors most highly valued in the organization. The Not-So-Hot Employee section provides contrast to the Star Employee section, giving the researcher greater context and insight with respect to understanding the overall company culture and conceptual distinctions or relevant boundaries of various themes or values. The Leadership Team is typically described as "having the qualities of star employees" plus a few other key features that distinguish them and shape the culture. The Interview 1 Worksheet and discussion of written responses is possibly the most important data mine in the CCVI process for obtaining a nearlycomprehensive list of concepts and language relating to company values and assessing Core Value Candidates in the CCVI Technique. It also requires the most time and largest amount of mental energy from interviewees in the initial round of interviews. 
Interview Question \#3: Open ended question mining for missing Core Value

Candidates. Interview Question 3 is a simple, open-ended question, "Is there anything else you'd like to tell me? Anything I'm missing or not asking about correctly that you would think is important for me to understand?" The goal of this question is simply to give the interviewee an opportunity to reflect and voice any important relevant themes or concepts not discussed so far in the interview process. This question is asked in every interview to ensure the completeness and accuracy of data collection.

Interview Question \#4: Mining for High-potential Core Value Candidates + "Ending on a high note." Interview Question 4 is the last interview question, and has two objectives: to take one last attempt at mining for important themes or values while also elevating the mood and mental energy of interviewees at the end of the interview. Interview Question 4 is, "What would you say is the best part about working here that someone like me might not be able to figure out by spending a week here observing or asking good questions - what's something that I might miss?" This question resembles Interview Question 3 in that the purpose is to uncover important relevant themes or concepts not discussed. But it is not open-ended and takes a specific, positive tack. This question serves to erase any possible experience of mental fatigue resulting from prior questions by ending the interview on a positive note - leaving the interviewee with an overall positive impression of the interaction.

Administering the Organizational Culture Assessment Instrument (OCAl). The Organizational Culture Assessment Instrument (Appendix G) is the most common assessment in the world for assessing organizational culture, and plays a central role in 
creating a Culture Profile at a company. Administer the OCAI by handing interviewees

the printed assessment during the interview (including the cover page with

instructions), and verbally outlining the instructions for participants to ensure

comprehension. In this research the following excerpts from the standard OCAI

instructions were read to participants to ensure comprehension:

The purpose of the OCAl is to assess six key dimensions of organizational culture. In completing the instrument, you will be providing a picture of how your organization operates and the values that characterize it. There are only 2 pages, and six questions. Each question has four alternatives. Divide 100 points among these four alternatives depending on the extent to which each alternative is similar to your own organization. Give a higher number of points to the alternative that is most similar to your organization. Just be sure your total equals 100 points for each question.

Note, that you will make 2 passes through these questions. On the first pass through the six questions complete the column labeled "Now." This refers to the culture, as it exists today. After you complete the "Now," you will find the questions repeated under a heading of "Preferred." Your answers to these questions should be based on how you would like the organization to look five years from now.

There are no right or wrong answers for these questions, just be as accurate as you can in responding to the questions so that your resulting cultural diagnosis will be as precise as possible.

It is appropriate to end audio recording when administering the OCAI, as no further mineable data is expected from the interview transcript after the last interview question. In the event that any useful conversation does arise during or after OCAI, two viable options for capture are to begin recording again or take notes that can be included as field notes. 
4.2 CCVI Step 2: Participant Observation. Conducting Participant Observation in order to gain perspective on company culture and values.

The second step in the CCVI process is conducting Participant Observation at the subject company. One work week (five days) of participant observation was completed at each company in this research to gain perspective on organizational culture and core values through immersion and direct observation of material culture, employee behavior and work processes. The essential activities involved are first to establish the scope and schedule for observations, then to introduce the researcher to participants, and finally to record field notes on observations (See Participant Observation Field Guide in Appendix H).

Establish Scope \& Schedule. Before beginning participant observation an appropriate timeframe and schedule should be agreed on between the researcher and company leadership. For the small companies included in this research (small, singlesite companies ranging from 18 to 50 employees), 4 to 5 work days were sufficient. All meetings or events that would be appropriate for the researcher to attend during the observation period are added to the observation schedule.

Introduction to participants. Company leadership must announce or introduce the researcher before beginning participant observation to obtain informed consent and prime employee expectations. The quality of the introduction will affect the quality of the participant observation experience. The researcher is best served by being understood as a friendly visitor interested in learning about the company culture and 
values. If employees are encouraged or made to feel comfortable sharing their perspectives voluntarily, the quality and usefulness of field notes will increase.

Observation \& Field Notes. The central activity in the Participant Observation step is the researcher maintaining a presence on site at a target company, recording relevant observations. The researcher attends meetings, inspects company physical layout and material culture ${ }^{16}$, observes employees, work groups and work processes, and records observations in the form of brief field notes. Resulting field notes include written descriptions of material culture, meetings, interactions, behaviors or processes that can be used to produce insight into the culture or core values of the target company. The week of observation at each target company was the most laborintensive week of the CCVI process, resulting from three to four hours spent formally writing up and analyzing field notes each day. Eight hours of observation followed by these written observations provide a means for uncovering new themes during data analysis and play a key role in supporting or contrasting with behaviors or values described during interviews.

\subsection{Step 3: Document Review. Conducting a Review of Company Documents in} order to gain perspective on company values.

The third step in the CCVI process is conducting a review of company documents which articulate company values. The set of documents appropriate for review should be agreed upon between the researcher and leadership. The documents must then be

\footnotetext{
${ }^{16}$ Material culture in this research refers to the physical objects, structures and spaces that shape and reflect culture, such as the status symbols embedded in offices, hallways, meeting rooms or workspaces.
} 
reviewed, coded, and included as items for analysis along with interview and observation data. This should include any pre-existing values statements, and may also include employee evaluations, employee handbook, performance evaluations, or any other materials that articulate company values. Pre-existing value statements in particular should factor significantly into the final presentation of results.

Effective handling of Pre-existing Values statements. Regardless of the format or specific content of pre-existing value statements, they are an obvious and essential candidate for inclusion in document review. The effective handling of pre-existing value statements is critical to successful acceptance of the results during final presentations because they are the most obvious benchmark to measure the applied CCVI results against. Practitioners must take care to investigate and respect the pre-existing statement by including core value candidates from pre-existing statements in the second round of interviews.

Language and values in pre-existing values statements should be treated as a stock of language and concepts for telling the tale of the core values uncovered by the applied CCVI without allowing the contents of the pre-existing values statements to disproportionately influence the applied CCVI results. This is because the purpose of the CCVI technique is to provide a fresh assessment of company core values, which may which may validate, replace or fall short of prior statements. 
Attention must also be given to distinguishing between core and other types of values ${ }^{17}$ in the second round of interviews and the final presentation. Examples of the effective handling of pre-existing values statements and distinguishing core from other types of values are discussed in section 6.3.

Performance Evaluations. The common use of performance evaluations in various organizations and their important role in the career progress of employees make these documents essential and relevant for review. Performance Evaluations should therefore be reviewed for articulations of company values or desired employee behaviors. Line items, words and phrases that reflect company values or desired behaviors can be useful later in the process when rendering Core Values statements in language that is familiar to employees during CCVI Step 6: Synthesis.

The CCVI Technique is designed to uncover specific behaviors associated with company core values for two main reasons: so that behaviors can be effortlessly included in Core Values statements and as line items Performance Evaluations. The suggestion to include the behaviors associated with company core values as line items in Performance Evaluations should also be made to companies during presentations if they are not present.

Employee Handbooks. Employee handbooks are often the first substantive company document given to new organizational members for review, and is therefore a chief candidate for document review. While Employee Handbooks are ubiquitous in

\footnotetext{
${ }^{17}$ Core and other important values are discussed in the literature review and shown in the Types of Organizational Values Chart in Appendix L.
} 
companies, the utility of those documents will vary. In the present research it was observed that Employee Handbooks may sometimes fall out of touch with reality and would therefore require significant updating before it could provide any real value to the investigation.

In response to this common occurrence, the researcher should collaborate with the leadership team in order to discern which documents are appropriate for review. To the degree that formal company documents are out of touch with current cultural reality, the researcher may opt to rely more heavily on the other two forms of primary data collection (interviews and participant observation) to accurately reflect the company culture.

4.4 CCVI Step 4: Initial Analysis. Conducting Initial Coding \& Analysis of collected data in order to create a Culture Profile and Core Value Candidates for use in Step 5: Second Semi-Structured Interviews.

The fourth step in the CCVI process is a critical step that produces the first major set of deliverables, marking the end of the first practitioner phase in the CCVI Technique. The overall goal of the Initial Analysis can be thought of as becoming completely prepared for the second round of interviews with company leaders through the coding and analysis of all collected data in order to create the culture profile and core value candidates used in the second set of semi-structured interviews with company leaders. The materials required to complete the second round of semistructured interviews are the deliverables produced in this step (See Table 4.4). 


\begin{tabular}{|l|l|l|}
\cline { 2 - 3 } \multicolumn{1}{c|}{} & \multicolumn{2}{c|}{ Phase 1 Deliverables } \\
\hline $\begin{array}{l}\text { Overall Goal: } \\
\text { "Create a..." }\end{array}$ & \multicolumn{1}{c|}{ Deliverable Name } & \multicolumn{1}{c|}{ Template Location } \\
\hline $\begin{array}{l}\text { Culture } \\
\text { Profile }\end{array}$ & 1) Overall Culture Assessment & Appendix J (OCAI Overall Results Template) \\
\cline { 2 - 4 } & 2) Itemized Culture Assessment & Appendix K (OCAl Itemized Results Template) \\
\hline $\begin{array}{l}\text { Core Value } \\
\text { Candidates }\end{array}$ & 3) Core Value Candidates & Appendix M (Core Values Identification Form) \\
\cline { 2 - 4 } & 4) Value-Behavior Association Form & Appendix N (Value-Behavior Association Form) \\
\hline
\end{tabular}

Table 4.4 First Milestone Deliverables for the CCVI Technique, resulting from the successful completion of Step 4: Initial Analysis.

Deliverable 1: An Overall Culture Assessment. The Overall Culture Assessment, based on OCAI Results for each interviewed leader, displays and compares their overall assessment of the company culture based on the CVF against the averaged OCAI results from all assessment participants (see Figure 10 for an example, or Appendix J for the worksheet template). The only data needed to produce this result is the OCAI data collected by administering the OCAl survey (Appendix G) at the end of each leader interview (Step 1). A separate Deliverable 1 must be created for each interviewee before their second interview because this document is used in the second interview. 
Overall Results from Organizational Culture Assessment Index (OCAI)

$\underline{\text { Your Overall OCAl Results }}$

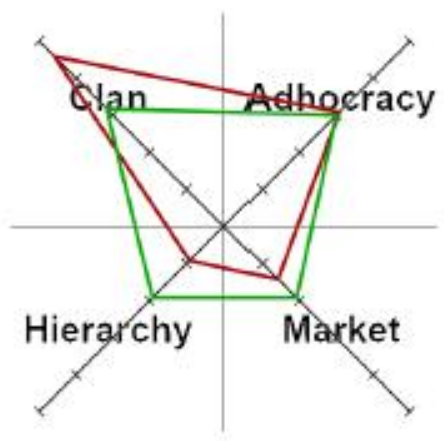

\begin{tabular}{|c|c|c|}
\hline & Now & Preferred \\
\hline Clan & 45.8 & 31.7 \\
\hline Adhocracy & 30.8 & 30 \\
\hline Market & 14.2 & 19.2 \\
\hline Hierarchy & 9.2 & 19.2 \\
\hline
\end{tabular}

\section{Leadership Team Average Overall OCAl}

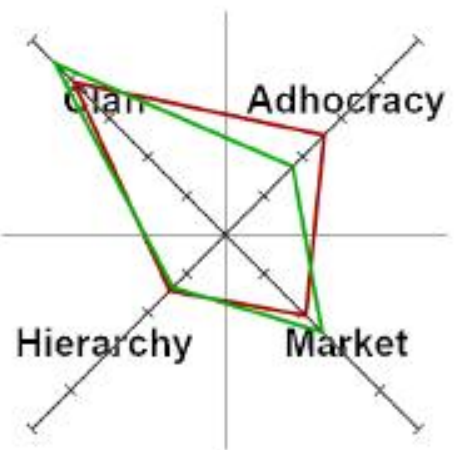

\begin{tabular}{|c|c|c|}
\hline & Now & Preferred \\
\hline Clan & 39.2 & 44 \\
\hline Adhocracy & 25.6 & 17.5 \\
\hline Market & 20.8 & 25 \\
\hline Hlerarchy & 14.4 & 13.6 \\
\hline
\end{tabular}

The Competing Values Framework

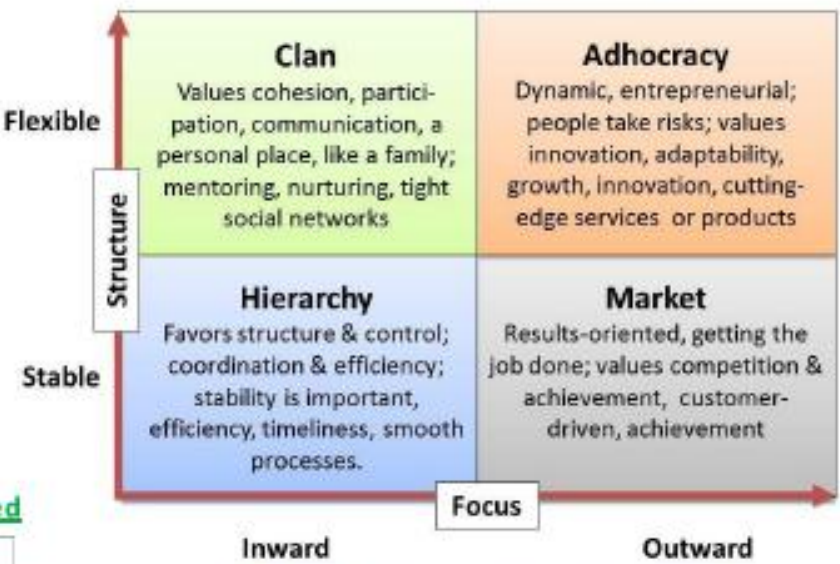

SOURCE: Greg Waddell, leadstrategic.com

The Competing Values Framework

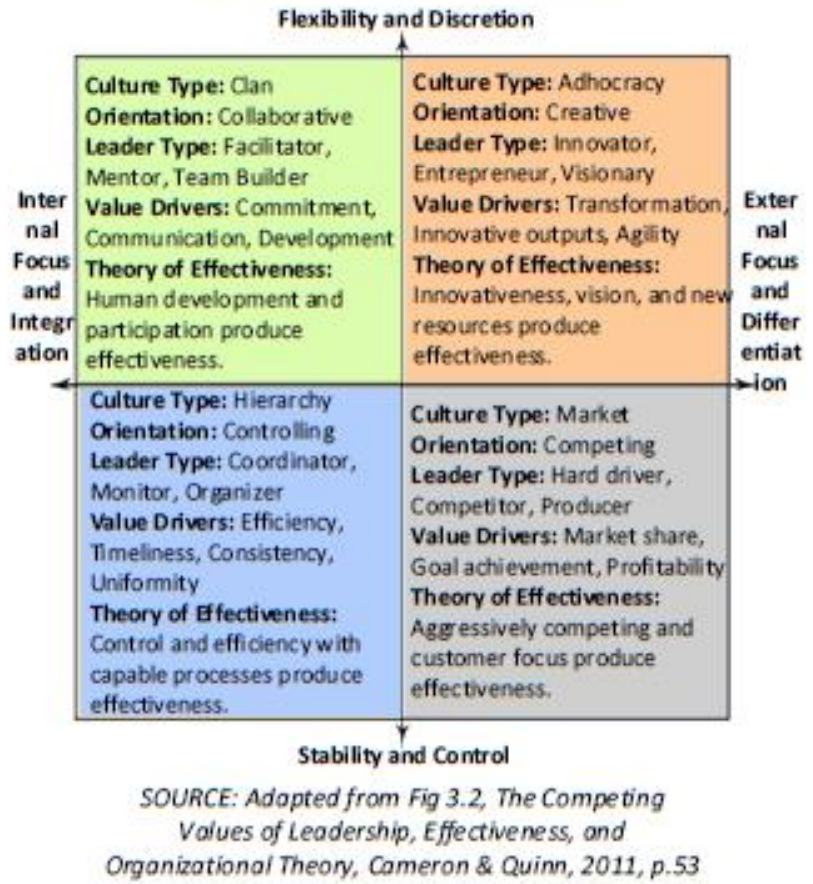

Figure 10: Overall Culture Assessment Example. See Appendix J for full-size template. 
Deliverable 2: Itemized Culture Assessment. The Itemized Culture Assessment, based on the OCAI Results for each interviewed leader, displays and compares their assessment of the company culture based on each of the six OCAI questions, and compares them against the averaged OCAI results for each question from all assessment participants (see Figure 11 for an example, or Appendix $\mathrm{K}$ for the worksheet template). The only data needed to produce this deliverable is the OCAI data collected by administering the OCAl survey (Appendix G) at the end of each leader interview (Step 1). An Itemized Culture Assessment must be created for each interviewee before their second interview because this document is used in the second interview. 


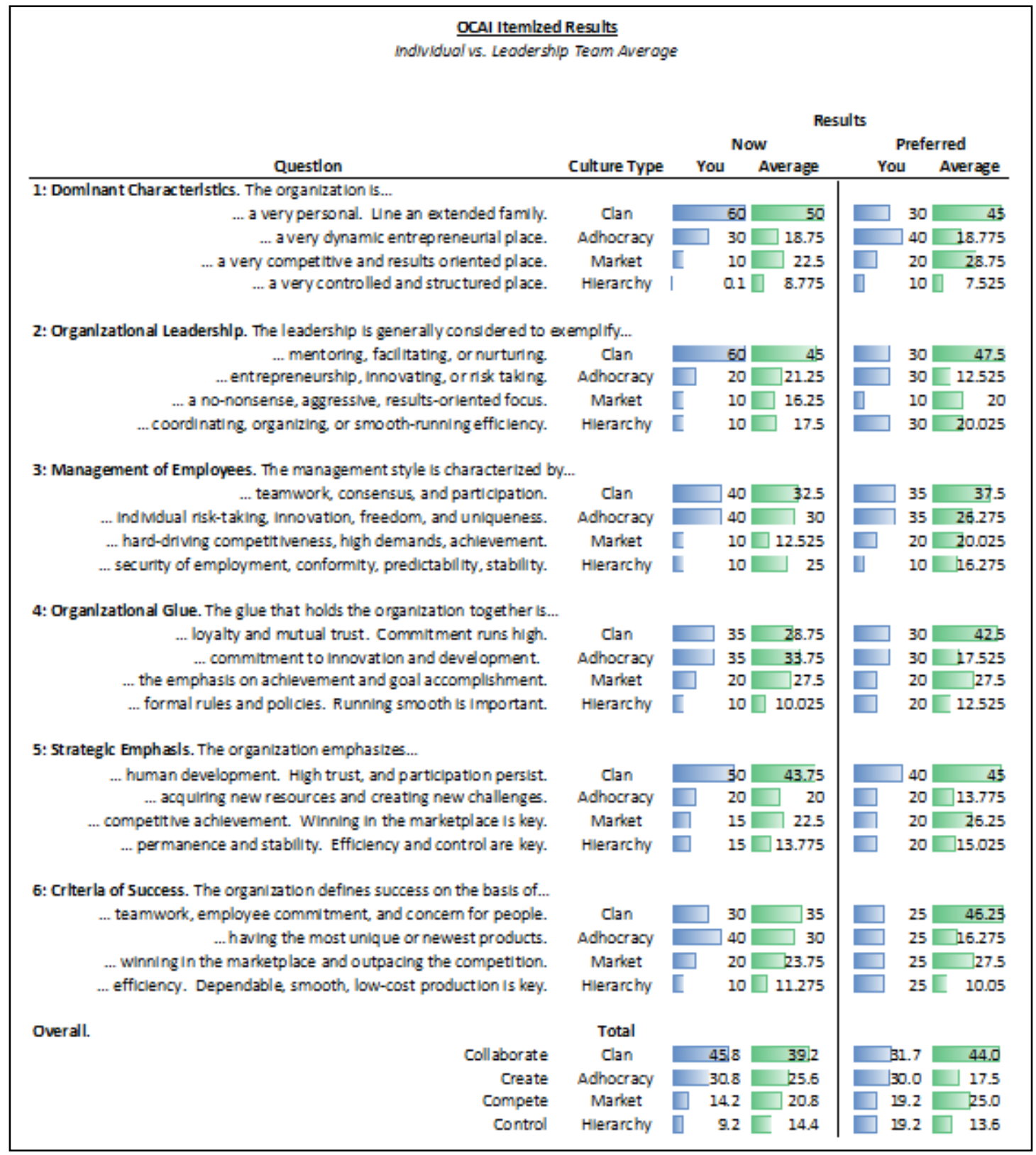

Figure 11: Itemized Culture Assessment Example. See Appendix K for full-size template.

Deliverable 3: Core Value Candidates on a Core Values Identification Form. In this research, the collection of organizational values from which core values may be selected at a target company are called "Core Value Candidates" in this research. A prepared list of Core Value Candidates on a Core Values Identification worksheet is a 
critical and labor-intensive deliverable used in second round interviews (see Figure 12

for an example, or Appendix $\mathrm{M}$ for the worksheet template). A separate copy of the

Core Value Identification Form is needed for each interviewee for their second

interview, but note that each copy is identical for interviewees.

Top Value Themes / Core Value Candidates

Servant's Heart: Service is at our core. We all enjoy knowing that our work is a benefit and delight to the next person downstream, whether that's another employee, another team, or the final customer. We don't aim to satisfice; we aim to exceed expectations.

Personal Growth: We have a lot of really talented employees. Really talented employees. This is because we are all learners. We believe that the willingness and ability to learn is more valuable than prior experience. If we are not advancing we are falling behind.

Problem Solving Prowess: What we do is solve problems for customers. And we love new challenges. We get bored easily. That's why we enjoy taking on jobs that others avoid or fail.

A Culture of Integrity, Maturity \& Respectfulness: Having a healthy culture allows [us] to thrive. We believe everything we do should be done well. We treat our customers, each other, and our community with respect. Drama, ego and paycheck mentalities don't belong here. We enjoy our work, and enjoy working together.

Do What You Love: We are engineers, pioneers, and craftsmen. Some of us love solving new problems every day. Some of us love building those solutions. Whether it is the challenge, the aesthetic, or the transformation of raw materials into a finished product, we love what we do.

Humble Talent: Our success depends on our ability to learn, adapt, and solve new problems. When talent loses humility, it loses those abilities. We believe overconfidence creates incompetence

Figure 12: Core Value Candidates Example. See Appendix M for full-size template. 
The list of Core Value Candidates included in this deliverable must be created by the analyst, and represents the best effort of the analyst to identify and organize the dominant trends in all collected data (from interviews, observations and document review) into a concise and comprehensive list of themes that articulate potential core values at the subject company. The construction of core value candidates begins with initial open coding of all collected data. The objective during this phase is to identify the contents in each segment of data to make them readily available for the subsequent thematic content analysis. Then the analyst must review all coded data and group together the repeated and emphasized ideas into concepts, and finally identify key themes in the data that emerge as an iterative and natural structuring of the data materializes. For further discussion of coding and initial analysis in this research, see section 6.4. For examples of tracing key themes from their grounding in raw data, see Appendix O.

Deliverable 4: Value-Behavior Association Form. A prepared list of behaviors on a Value-Behavior Association Form (see Figure 13 for an example, or Appendix $\mathrm{N}$ for the worksheet template) is the final deliverable from Initial Analysis to be included in the second round interviews. A separate copy is needed for each interviewee for their second interview, but note that each copy is identical for all interviewees. The list of behaviors included in this deliverable must be created by the analyst, in association with the Core Value Candidates on a Core Values Identification worksheet. In other words, data that refers to specific behaviors that may be connected to potential core values that are grouped together through coding and analysis will be used in association with 
each other in this deliverable, which is then used in second round interviews (for examples of tracing behaviors and core value candidates from their grounding in raw data, see Appendix O). 


\section{Value-Behavior Association Form (Example)}

Core values should provide a clear blueprint for employee behavior.

1. Review the trends in value candidates. Include any important ideas that are missing.

2. Within each value category, rank the top, most important behaviors at [COMPANY], in order of perceived importance (or impact on organizational effectiveness) with 1 being the most important. Please rank at least 4 in each category.

3. (Optional) Weight each of your ranked values and/or behaviors within categories. The total must equal $100 \%$

\section{Major Trends:}

Weight Ranking:

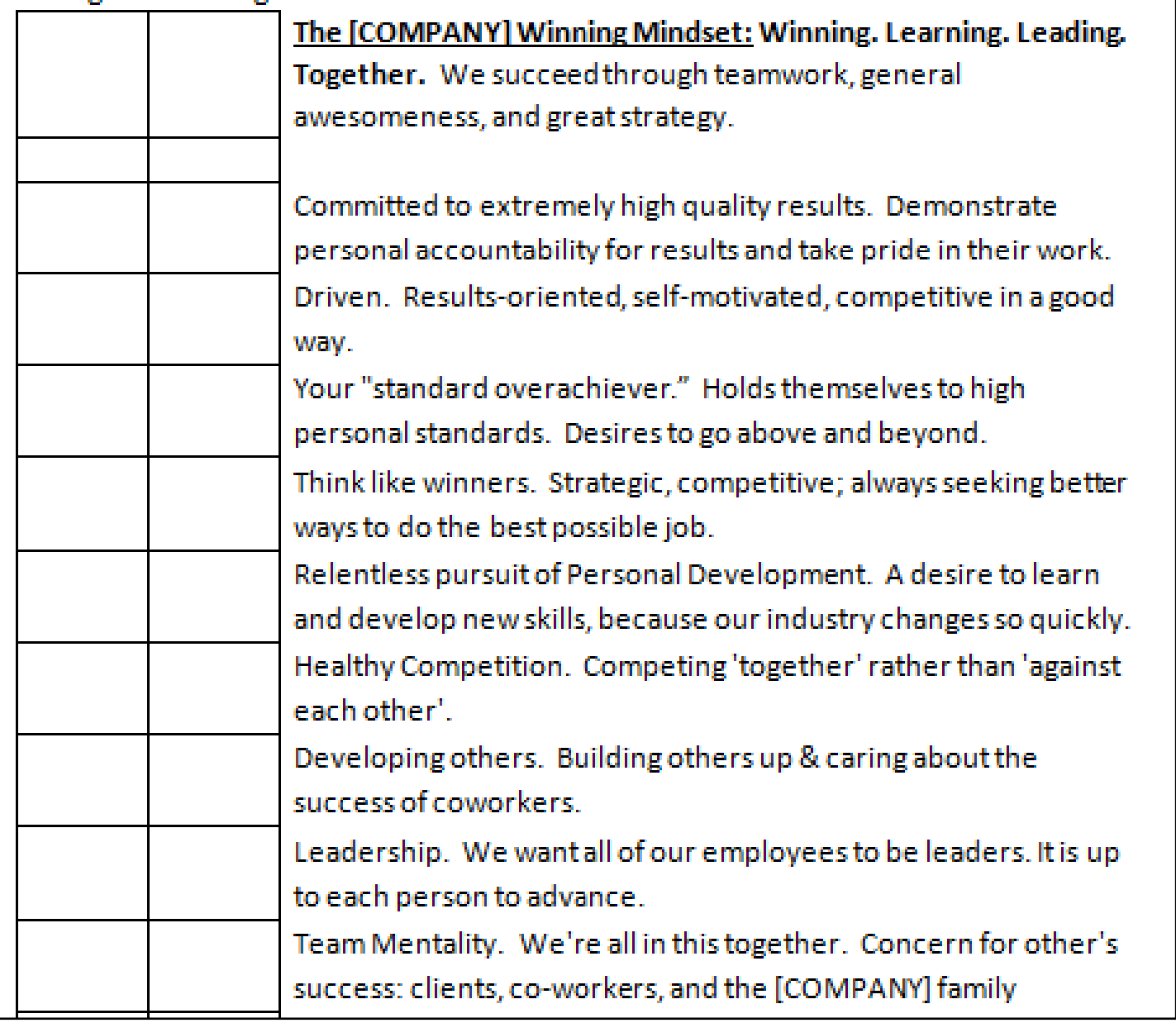

Figure 13: Core Value Candidates Example. Includes an image selection from the first page only. See Appendix $N$ for full-size template. 
Closing Thoughts on Step 4. The length of Initial Analysis in labor-hours will vary according to the talent and experience of the analyst(s), and the volume of data. Building flexibility and appropriate timelines for task completion into the schedule is key. An important intangible implicit in the Initial Coding and Analysis step of the CCVI Technique (Step 4) is gaining sufficient familiarity with the data to be able to comfortably guide discussion in the subsequent interviews (Step 5).

4.5 CCVI Step 5: Second Interviews. Conducting Semi-Structured Interviews in order to gain leadership perspectives on company culture and values.

The fifth step in the CCVI process is conducting a second round of one-on-one semi-structured interviews with each of the participating company leaders. The Second Interview Protocol (Appendix I) includes a list of materials to bring, interview questions, and the basic flow for these semi-structured interviews. These focused and fast-paced interviews yield high-quality data by guiding interviewees through a process of:

1) Familiarizing the interviewee with the Competing Values Framework

2) Sensitizing the interviewee to descriptions, perspectives and data points reflecting their organizational culture and values

3) Identifying organizational core values (from the interviewee's perspective)

4) Associating behaviors with each identified core value.

5) Discriminating between core and other types of organizational values.

The end result of this interview process will be, for each interviewee, a set of interviewee-identified core values for the company, a set of behaviors the interviewee associates with each identified core value, and a rich conversation for further transcription, coding and analysis by the researcher. The essential flowchart for Second 
Interviews can be seen in Figure 14, below. The second interviews can range in length from 45 to 60 minutes, depending on the brevity or nature of participants.

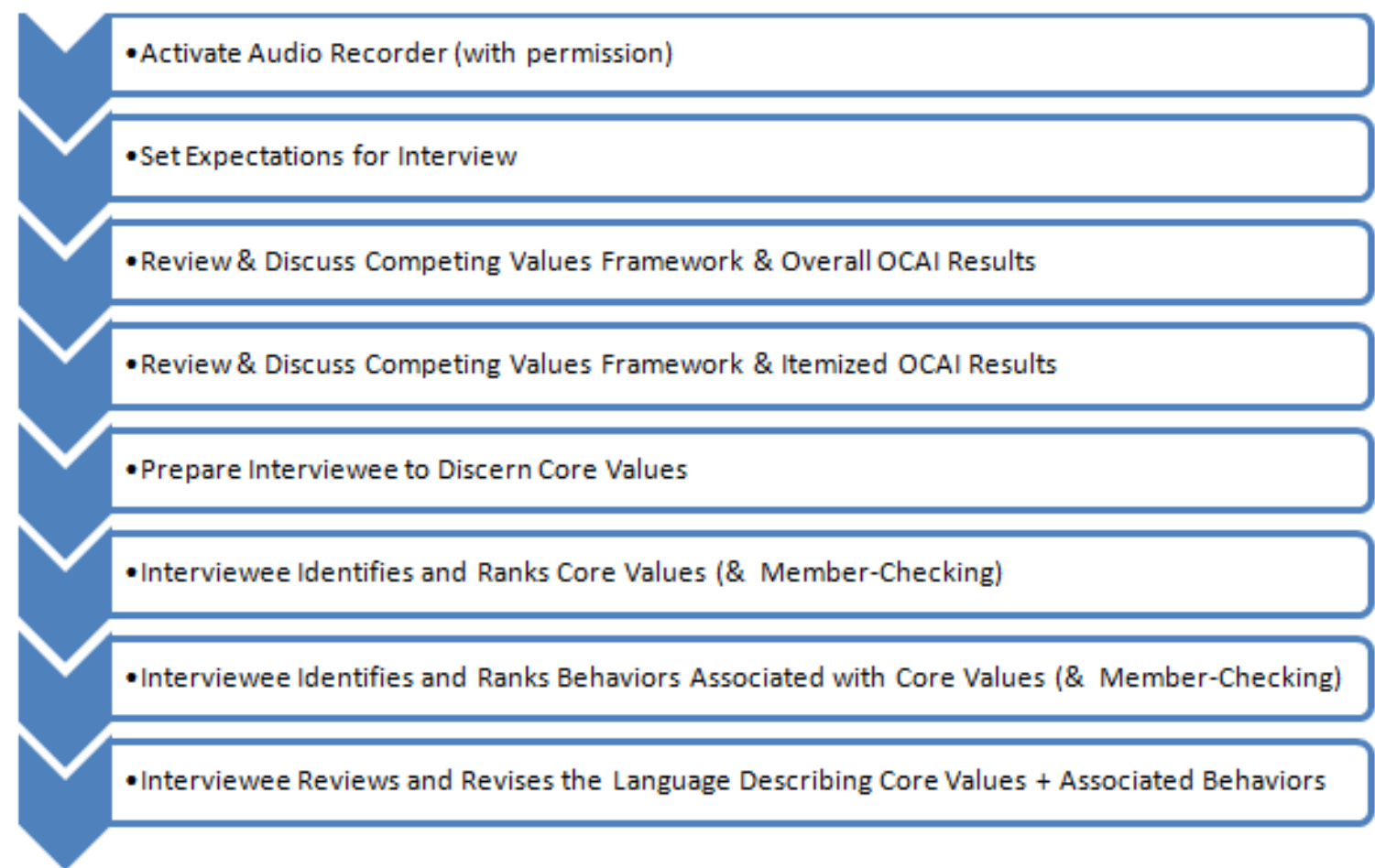

Figure 14: Flowchart for CCVI Technique Step 5: Second Interview Protocol Overview. See Appendix I for formal protocol.

Activate Audio Recorder (with permission). Request permission to record each interview from each interviewee, as per the Second Interview Protocol (Appendix I). Recording the interview is critical to the CCVI Technique because coding and analyzing interview transcripts play a key role in identifying themes and core value candidates, and crafting core values in the shared-language of participants. Proceed once permission to record is verbally obtained ${ }^{18}$.

\footnotetext{
${ }^{18}$ Note that proof of permission to record all interviews is also obtained in writing in the signed letter of consent obtained at beginning of the first interview.
} 
Set Expectations for Interview. Verbally recount the expected timeframe of the interview (one hour), the key objectives, and a basic overview of what the participant should expect, as per the Second Interview Protocol. The essential script used for this research was:

The goals of this meeting are:

1) to share with you the results of this study so far,

2) to complete an exercise that will help me gain a clearer picture of the cultural priorities, and,

3) for you that you come away refreshed and energized by this conversation.

This is going to be interesting, it won't take more than an hour, and if you want to decline any questions or anything like that once again it's totally okay. And we have a lot of material to cover so we're going to charge through.

Upon confirmation, proceed to the next step.

Review \& Discuss Competing Values Framework \& Overall OCAl Results. The next step is to review and discuss four component graphics of the Overall Culture Assessment worksheet: the interviewee's individual OCAI results, the group average OCAl results, and two CVF charts (see Figure 4.4a for an example, or Appendix J for full template). The essential process for completing this step is described below. Subtleties are described in greater detail and with illustrative examples from the field in the discussion chapter.

Starting with the individual OCAI results for the interviewee, at the top left of the OCAI Overall Results page, explain that "these are the results of the OCAI assessment you took," explain the meaning of the red and green chart lines (including dominant culture types, and highlight the preferred changes), and explain the meaning of the four 
culture quadrants which represent the four cultural archetypes in the CVF. Next, introduce the adjacent CVF figure (top right) to the interviewee, explaining that the four component quadrants of that figure correspond to the four quadrants in their OCAI results graph. Once interviewees grasp how to read their overall OCAI results and have read the CVF chart descriptions, probe for any interpretations or impressions that could enrich your data. For example, if the OCAI results suggest that the interviewee prefers that company shows a $40 \%$ increase in Hierarchy in the next 5 years, this is a great time to ask the interviewee to explain that result.

After probing for interpretations or impressions regarding the interviewee's individual OCAI results, introduce the averaged leadership team OCAI results (in the bottom left of the worksheet). Briefly describe the dominant features of these results (e.g., the dominant culture types, unexpected features, or important differences/similarities between the individual's OCAI results and the team), then introduce the adjacent CVF figure (bottom right), explaining that it provides more detail and description of the quadrants. See Chapter 6 for more discussion regarding the important role of including an abundance of CVF description and language throughout the results-discussion process, which serves to sensitize the interviewee to CVF concepts and aids the researcher in identifying the themes and language of the CVF most fitting for the target company. Once interviewees grasp how to read their Overall Culture Assessment page and have read the CVF chart descriptions, discuss all results and allow conversation to flow naturally, following the interviewees' lead as they take in the information. Probe for any interpretations or impressions that could enrich the data. 
Review \& Discuss Competing Values Framework \& Itemized OCAI Results. The Itemized Culture Assessment worksheet displays and compares their assessment of the company culture based on each of the six OCAl questions, and compares them against the averaged OCAI results for each question from all assessment participants (see Figure 4.4b for an example, or Appendix K for the worksheet template). Quickly review and discuss the worksheet with the interviewee, highlighting key trends you identified during your analysis of the data. Allow conversation to flow naturally, and probe for any interpretations or impressions that could enrich the data.

Interviewee Identifies and Ranks Core Values \& Member-Checking. Two worksheets are used in the CCVI Technique to select core values during second round interviews. First, train interviewees how to distinguish between core and other types of values by quickly reviewing the Types of Organizational Values Chart (Appendix L). Next, review and discuss the prepared Core Values Identification worksheet (see Figure 4.4c for an example, or Appendix $\mathrm{M}$ for worksheet template). A separate copy of the Core Values Identification worksheet is needed for each interviewee for their second interview, but note that each copy is identical for interviewees. Member-check by asking interviewee if anything important is missing from this worksheet (which should contain a concise and comprehensive list of themes that articulate potential core values at the subject company based on all previously collected data). Then ask the interviewee to identify their perceived core values. Allow discussion to flow naturally. Finally, ask interviewee to rank or prioritize the core value candidates. Weightings can 
be included. The discussion and ranking results from this step play a central role in organizing the final assessment of company Core Values.

Interviewee Identifies and Ranks Behaviors Associated with Core Values. Present the interviewee with a Value-Behavior Association Form (see Figure 4.4d for an example, or Appendix $\mathrm{N}$ for the worksheet template). A separate copy is needed for each interviewee for their second interview, but note that each copy is identical for all interviewees. The form includes a prepared list of behaviors that can be associated with core values. Instruct interviewee to review the content, include any items or behaviors that may be missing, and then rank the top, most important behaviors within each category in order of perceived importance. This process yields a prioritized list of behaviors that can be associated with each selected core value.

Interviewee Reviews and Revises the Language Describing Core Values and Associated Behaviors. The final task in the second round of interviews is to allow interviewees to carefully review the language used in describing important core value candidates and associated behaviors and make any suggestions. Some interviewees will prefer to complete this task during the final minutes of the interview (usually in silence), while others will prefer to review it in solitude at a later time and follow up the next day. Discern and accommodate whichever style is most appropriate for each interviewee.

4.6 CCVI Step 6: Data Synthesis. Conducting Final Coding \& Analysis of collected data in order to create a written statement of company Core Values. 
The sixth step in the CCVI process is a critical step that produces the second major deliverable, a written statement of company Core Values, marking the end of the second practitioner phase in the CCVI Technique. The essential process of CCVI Step 6, Data Synthesis, is to synthesize the feedback from the previous round of interviews in order to structure the resulting Core Values statement. The overall goal of the Data Synthesis can be thought of as constructing a data-driven description of organizational core values through the coding and analysis of the collected data. The two core tasks involved in preparing the document are organizing core values based on feedback, and organizing descriptive language and behaviors associated with each core values.

Task 1: Identifying and Ordering Core Values based on feedback. The first task is to review and synthesize collected data in order to give reliable ranking or structure to top organizational values and select organizational core values. Core values are identified through the analysis of the rankings given to core value candidates on the Core Values Identification forms, and the Core Values identification discussion from the second round of interviews (which included discussion of all prior data and analysis). The ease of core value identification and ordering analysis is contingent on the quality and clarity of the Core Values Identification discussion data. For example, the selection of core values may depended more heavily on Core Values Identification discussion data if the rankings generated by the leadership team are highly variable (See Table 1, below for an illustration). 


\begin{tabular}{|r|r|r|r|r|r|l|}
\hline \multicolumn{6}{|c|}{ Individual Leader Rankings } & \multicolumn{2}{l|}{$\begin{array}{l}\text { Average } \\
\text { A }\end{array}$ B } & \multicolumn{1}{c|}{ C } & \multicolumn{1}{c|}{ D } & \multicolumn{1}{c|}{ E } & Rankings & Core Value Candidates: \\
\hline 3 & 1 & 3 & 2 & 1 & 2 & Do what you love \\
\hline 1 & 6 & 2 & 1 & 3 & 2.6 & Problem Solving DNA \\
\hline 5 & 4 & 1 & 4 & 2 & 3.2 & Culture of Integrity, Maturity \& Respect \\
\hline 4 & 2 & 4 & 5 & 4 & 3.8 & Serving Through Work Well Done \\
\hline 2 & 3 & 5 & 3 & 6 & 3.8 & Humble Confidence \\
\hline 6 & 5 & 6 & 6 & 5 & 5.6 & Personal Growth \\
\hline
\end{tabular}

Table 1: An Example of Core Values Synthesis. Table reflects an example of highly variable ranking data collected through the Core Values Identification forms completed by five individual leaders at one company.

The identification of core values in such cases may depend more heavily on analysis of discussion data than the value rankings data. Conclusions regarding the data reflected in Table 1 are described in section 6.6.

Task 2: Organizing descriptive language and behaviors associated with each core value. The second task required to complete the Core Values document needed in the final interviews is organizing descriptive language and behaviors associated with each core value. This task is achieved by analyzing and synthesizing all collected data, focusing particularly on the completed Value-Behavior Association forms from second interviews, the language refinements and suggestions collected at the end of second interviews, and all relevant data, discussion or analysis. In this research, the organization and contents of Core Value Statements were generated using spreadsheets containing all relevant data and language, and were constructed and iteratively condensed through synthesis of similar and well supported concepts (See Figure 15 for an example of this process). The end result is the second major deliverable: a concise, carefully structured and data-driven statement of company Core Values with associated 
behaviors to be used in the third round of interviews (See Figure 15 for an example

below).

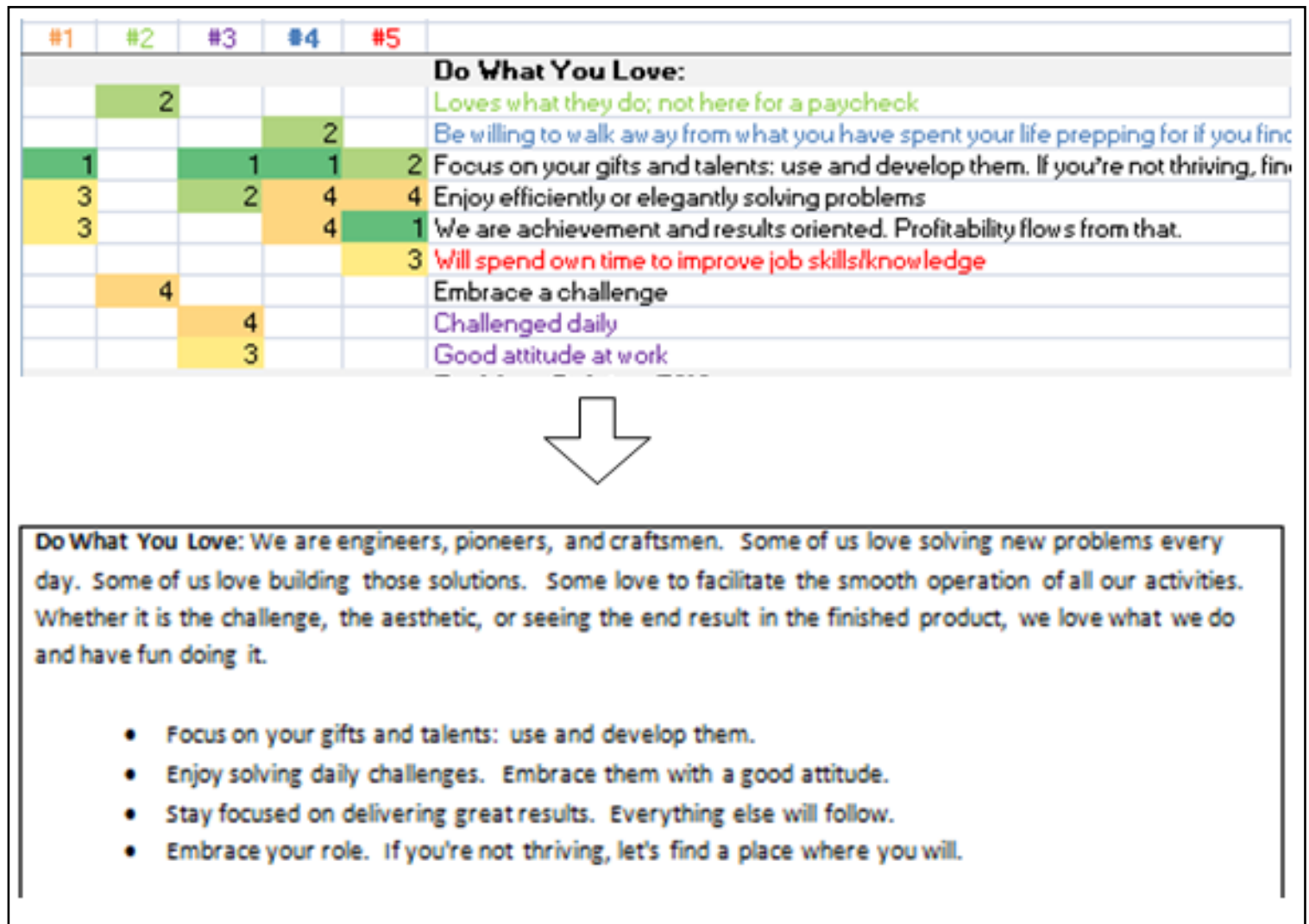

Figure 15: An Example of Synthesis. Figure shows a spreadsheet (top) used to organize and synthesize feedback on Company $B$ descriptive language and associated behaviors from five leaders (based primarily on collected Value-Behavior Association Forms) for the "Do What You Love" core value. Rank values were displayed and newly contributed language was color coded to match the original source and rank values to assist the analyst in visually grasping complete and contextualized data throughout the analysis. A complete statement of the core value is pictured below, which resulted from the synthesis process. Note that the Core Values Identification Forms, which also contain a large amount of critical language involved in this process, are not explicitly shown in this example.

\subsection{Step 7: Final Interviews}

The seventh step in the CCVI Technique is a final round of interviews with participating company leaders. The two components of final interviews are memberchecking and to discuss any questions or suggestions the practitioner wants to test or explore before the final presentation. 
Member-checking in the final interviews means gaining testimonial validity regarding the completeness and accuracy of the Core Values statement - and to obtain any feedback necessary for the subsequent presentation of results. The process for member-checking in final interviews is to first provide the interviewee with the Core Values statement constructed in CCVI step 6 for review, and then probe for any feedback regarding the completeness and correctness of the document. This is an open-ended process that allows interviewees to reflect and respond freely. Any feedback offered by participants that would impact the final presentation should be used to make adjustments.

This third and final round of semi-structured interviews also presents an opportunity to discuss any questions or suggestions the practitioner wants to test or explore before the final presentation. For example, the final round of interviews can be used to ask about specific, practical suggestions you may have for improving policy and core values alignment, how to improve specific processes you became familiar with during the research, or suggestions on how to close the gap between the current culture and preferred future depicted by the OCAI results. Investigation of desired organizational change and making recommendations for improvement marks the boundary of the CCVI Technique, which is highly compatible with this larger process.

\subsection{Step 8: Presentation of Results}

The final step in the CCVI Technique is the presentation of results to the relevant audience and discussion of applied opportunities for guiding organizational change. The scope of the CCVI in assessing of core values for guiding organizational change ends with 
the final presentation of company results. Once a Core Values statement has been produced, verified and validated by company leadership, results are submitted to decision makers. Rich data for guiding organizational change has been made available to the researcher throughout this process, and suggestions on how to guide organizational change based on the assessment are submitted along with the core values assessment. In this research, presentations were made to the entire leadership team for each participating company. The purpose of these presentations was to transfer the results and insights from the applied CCVI Technique to the people able to effectively carry out organizational changed at each organization.

For practitioners seeking to use the CCVI Technique to its full potential (both assessing core values and following through with a plan for guiding organizational culture change), there are two major opportunities inherent in the technique for deriving suggestions for guiding organizational change. The first is taking care during interviews, observation and document review to actively question and pursue opportunities for improvement. The second major opportunity is utilizing the vast research available on guiding organizational change based on the Competing Values Framework. For example, Cameron and Quinn (2013) provide generic sets of specific suggestions and improvements that can be made at organizations seeking organizational change. The level of practitioner familiarity with target companies yielded through application of the CCVI Technique allows the practitioner to select generic organizational change tactics or concepts from that manual (or others) and customize them for application at the target company. These steps were taken to 
provide greater value to each target company during presentations. This also served to validate the usefulness of the CCVI Technique in guiding organizational culture change. 


\section{Chapter 5: Company-Specific Results}

This chapter describes the core values identified at each subject company included in this research. These results are unique to each company and are therefore not intended for transfer to other organizations. The purpose of this chapter is to satisfy A-RQ\#1 (What are the unique core values at each subject company?) and to give examples of what completed core values statements resulting from the applied CCVI Technique look like. The sections of this chapter have an intentionally modular design, in order to maximize the ability of a reader to examine only sections of interest with minimal dependence on prior or subsequent material.

\subsection{Introduction to Company A Case Study Results}

The primary results discussed in this section are the unique core values identified at Company A, which are "Delivering Results," "Integrity," and "Developing our People" (See Figure 16 for complete Statement of Company A core values). 


\section{Delivering Results}

We strive to achieve excellent results.

- Bringing a positive, can-do attitude to every challenge is the key to our success.

- We accept personal responsibility and accountability.

- We work hard \& smart.

Integrity

Honesty in all we do.

- We must always walk the talk, own our actions and take responsibility.

- Do work to the best of your ability and to the highest quality.

- We don't take shortcuts. We do it the right way.

\section{Developing our People}

We are all craftsmen, mentors and teammates. We help each other improve every day. We do this because we honestly care about everyone's personal growth and it makes us more effective.

- Be willing to listen and learn.

- Help and train each other.

- Askquestions.

Figure 16: Company A Results: Core Values. Figure includes the full text of the identified core values and associated behaviors as identified through the applied technique and validated by study participants.

The essential narrative, validated by participants after the presentation of results, is that Company $A$ succeeds in their specific high-quality niche because they deliver unusually high-quality results (Core Value \#1), with a level of expertise, thoroughness and correctness (Core Value \#2) that is only possible through the culture of continuously developing high-quality workers (Core Value \#3), which competitors typically fail to maintain. There is a general trend in the blue-collar, Company A industry to "cheat" or "cut corners." Company A succeeds through operationalizing their core values, which means continually disabusing new employees of bad habits from the industry, prioritizing integrity, producing quality results, and then gaining outstanding 
relationships and reputation as a result. While elements of these results may resemble other organizations, these results are non-transferable.

\subsubsection{Company A, Core Value 1: Delivering Results.}

The first core value, "Delivering Results," is comprised of five major components. The constituent themes, including associated behaviors, together form what study participants call "Delivering Results." The strong results orientation at Company A was driven primarily by the company owner, who systematically operationalizes and reinforces this core values through the management of human resources and business processes. The specific conceptual architecture of this core value resulted from application of the CCVI Technique and is captured in the top part of Figure 16.

Delivering Results was described by study participants as the most important value. All text, concepts, relationships or associations presented here were validated by study participants during the final presentation. This core value relates directly to the dominant "Market" culture type of Company A, which was identified through the analysis of collected interview, observation and document review data and validated by company leadership. Company A participants hold the belief that goal achievement and profitability play the largest role in their success. For more detailed understanding of the major conceptual components underlying this core value, a technical appendix table has been prepared (Appendix $\mathrm{O}$, Table 4) that traces this core value and major conceptual components back to examples of original precedents in the data.

5.1.2 Company A, Core Value 2: Integrity. 
The second core value at Company A, "Integrity," is comprised of four constituent themes, including associated behaviors, which together form what study participants call "Integrity." The specific conceptual architecture of the Integrity core value, which resulted from application of the CCVI Technique, is captured in the middle portion of Figure 16.

The four major conceptual components of the "Integrity" core value at Company A are traced back to examples of original precedents in Appendix 0, Table 5. An important feature of "Integrity" is that this value was prominently displayed at the top of Company A's preexisting statement of Core Values established many years prior to this study, which is also documented in Appendix 0 , Table 5.

The "Integrity" core value was described and confirmed by study participants as the embodiment of an underlying belief that Company A succeeds in their specific highquality niche because they deliver unusually high-quality results with a level of thoroughness and correctness (Core Value \#2) that competitors typically fail to maintain. There is a general trend in the Company A blue-collar industry to "cheat" or "cut corners." Company A succeeds through operationalizing their "Integrity" core value, which means continually disabusing new employees of bad habits from the industry, prioritizing integrity, training integrity, producing true quality results, and thereby gaining outstanding relationships and reputation which enable profitability.

\subsubsection{Company A, Core Value 3: Developing our People.}


The third core value at Company A, "Developing our People," is a core value that was missing from the preexisting statement of core values at Company A. Study participants confirmed that this core value plays a critical role in increasing or maintaining the competitive market advantage in the industry niche Company $\mathrm{A}$ occupies because the quality of results required for success at Company A cannot be achieved or sustained without significant investment in the skills and work habits of employees. The mission-critical nature elevates the concept of balanced technical and people skills to the level of core value. Study participants also acknowledged specific opportunities for improving company performance through more complete and systematic applications of this core value that may have been historically overlooked. The specific conceptual architecture of this Developing our People, which resulted from application of the CCVI Technique, is captured in the bottom part of Figure 16.

Several elements of "Developing our People" were adapted from the original preexisting values statement at Company A to form the original thematic concept in the initial data analysis phase. The high priority placed on "Developing our People" was formalized during the second Semi-structured Interview (Step 5 of the CCVI Technique) and validated as a core value during final interviews and the presentation. The data in Appendix 0 , Table 6 support these results, and trace this core value and major conceptual components back to examples of original precedents in the data.

The "Developing our People" core value at Company A was described by multiple company leaders as contributing directly to the market-share potential and goalachievement focus characteristic of the dominant "Market" culture. However, 
"Developing our People" was not historically considered a core value. Participants acknowledged that the short-term performance focus also characteristic of Marketdominant cultures may have contributed to the historic lack of acknowledgement at Company A regarding this long-term oriented "Developing our People" core value. These realizations were obtained most powerfully during second round interviews (Step 5) and the final presentation (Step 8) of the CCVI process, where themes, trends, and values were rendered most accessible for study participants. The balance between short-term and long-term performance is considered by participants to be vital to company long-term success and maintenance of the Company A competitive advantage. All text, concepts, pictures, relationships or associations presented here were validated by study participants during the final presentation.

\subsection{Introduction to Company B Case Study Results}

The primary results discussed in this section are the unique core values identified at Company $\mathrm{B}$, which address the applied research question for Company $\mathrm{B}(A-R Q \# 1$ : What are the unique core values at each subject company?). The unique core values at Company B include "Do What You Love," "Problem Solving DNA," and "A Culture of Integrity, Maturity \& Respect" (See Figure 17 for complete statement of Company B core values). Each core value is embedded within elaborate prose for clarifying the socially-shared meaning of each value for participants. Associated behaviors were also constructed from company data to accompany the brief core value statements to provide further clarity. The Core Values, descriptive text and associated behaviors are 
framed in language that was co-constructed with the leadership team of Company B

through application of the CCVI Technique.

Do What You Love: We are engineers, pioneers, and craftsmen. Some of us love solving new problems every day. Some of us love building those solutions. Some love to facilitate the smooth operation of all our activities. Whether it is the challenge, the aesthetic, or seeing the end result in the finished product, we love what we do and have fun doing it.

- Focus on your gifts and talents: use and develop them.

- Enjoy solving daily challenges. Embrace them with a good attitude.

- Stay focused on delivering great results. Everything else will follow.

- Embrace your role. If you're not thriving, let's find a place where you will.

Problem Solving DNA: What we do is solve problems for customers. So we hire and develop people who have the problem solving DNA and the perseverance to press forward through challenges. We look for ways to improve and identify potential problems before they develop. We love new challenges and coming up with unique solutions. That's why we enjoy taking on jobs that others fail or avoid.

- Demonstrate the DNA. Use your gifts and abilities to find and fix problems.

- Identify potential problems before they show up.

- Show a sense of priority. Solve the most important problems first.

- Don't be afraid to dig in, learn, and ask questions.

- Persevere. Persistence is key.

A Culture of Integrity, Maturity \& Respectfulness: Having a healthy culture allows us to thrive. We believe everything we do should be done well. We treat our customers, each other, and our community with respect. Any rudeness, crudeness, profanity, drama or ego is out of place here. We enjoy our work, and enjoy working together as a team.

- Treat others with dignity and respect.

- Always do the right thing. No short cuts.

- Work hard. There is immense dignity in a taskwell done.

- Dowhatever is needed, even if it is inconvenient.

- Be a team player. None of us are perfect. But together we surpass our individual limits.

Figure 17: Company B Results: Core Values. Figure includes the full text of the identified core values and associated behaviors as identified through the applied technique and validated by study participants. 
Application of the CCVI Technique at Company B yielded 6 core value candidates in the initial Analysis phase (Step 4). Subsequent discussion and ranking of core value candidates with company leadership distinguished three core values at Company B from the other core value candidates (see Table 2 for ranking results). These results were validated by company leadership after presentation.

\begin{tabular}{|r|r|r|r|r|r|l|}
\hline \multicolumn{6}{|c|}{ Individual Leader Rankings } & \multicolumn{2}{l|}{$\begin{array}{l}\text { Average } \\
\text { Rankings }\end{array}$} & Core Value Candidates: \\
\hline A & \multicolumn{1}{c|}{ B } & \multicolumn{1}{c|}{ C } & \multicolumn{1}{c|}{ D } & \multicolumn{1}{c|}{ E } & Ran \\
\hline 3 & 1 & 3 & 2 & 1 & 2 & Do what you love \\
\hline 1 & 6 & 2 & 1 & 3 & 2.6 & Problem Solving DNA \\
\hline 5 & 4 & 1 & 4 & 2 & 3.2 & Culture of Integrity, Maturity \& Respect \\
\hline 4 & 2 & 4 & 5 & 4 & 3.8 & Serving Through Work Well Done \\
\hline 2 & 3 & 5 & 3 & 6 & 3.8 & Humble Confidence \\
\hline 6 & 5 & 6 & 6 & 5 & 5.6 & Personal Growth \\
\hline
\end{tabular}

Table 2: Company B Core Value Candidates. Figure includes the top six, most important values at Company $B$, including and distinguishing between the three identified core values and other important values (in grey). The three core values were initially distinguished through ranking and discussion, and the distinction validated after presentation.

These three core values together form a cohesive cultural foundation necessary for Company B success. Company B leadership at describe the core business function in this way: "what we do is solve problems for clients." Therefore, it is essential for employees to have and demonstrate "Problem Solving DNA" (core value \#2). Because Company B specializes in solving unique, small batch, extremely difficult problems, it is also required that employees not only have high potential for problem solving, but also that they are passionate about their craft in order to continually increase competence. The ubiquitous and requisite passion for the work at Company B is expressed as "Do what you love" (core value \#1). Finally, in order for the extremely high levels of competence and continuous personal growth to exist and thrive at Company $B$, the 
leadership team also identified a "Culture of Integrity, Maturity \& Respect" (core value \#3) as necessary. The Company B cultural value (core value \#3) enables the competence and personal growth related core values of "Problem Solving DNA" and "Do what you love." Each Company B core value is presented in sections 5.2.1 - 5.2.3 below, along with a sampling of the data from which they were derived. Company B core values can be traced back through major themes and concepts to precedents in the data in Appendix O, Table 7 through Table 9.

\subsubsection{Company B, Core Value 1: Do What You Love.}

The first core value is comprised of thick descriptive text and four associated behaviors that together form what study participants call "Do What You Love" (see Figure 17). The high requirement for increasing levels of competence and achievement at Company B make personal growth necessary for success at the company. Passion for the work was identified as the critical element which enables the high achievement and continuous growth, which is here expressed at Company B in the imperative: "Do What You Love." This specific language and conceptual architecture of this core value, which resulted from application of the CCVI Technique, is captured in the top portion of Figure 17.

The core value "Do What You Love" relates directly to the dominant "Adhocracy" culture type of Company B, which was identified through the analysis of collected interview, observation and document review data and validated by company leadership. Company B participants hold the belief that success requires innovation and cutting edge achievement, and that individual passion is required to continually remain on the 
cutting edge. Appendix 0 , Table 7 traces this core value and major conceptual components back to examples of original precedents in the data. "Do What You Love" was described by study participants as the most important value. All text, concepts, relationships or associations presented here were validated by study participants during the presentation.

\subsubsection{Company B, Core Value 2: Problem Solving DNA}

The second core value at Company B is comprised of descriptive text and associated behaviors derived from major related themes, which together form what study participants call "Problem Solving DNA." This specific conceptual architecture of the Problem Solving DNA core value, which resulted from application of the CCVI Technique, is captured in the middle portion of Figure 17.

The "Problem Solving DNA" core value was independently described as critical to the success of Company B by all company leaders, as there is a shared-understanding that the core business function of Company B is solving problems for clients. The major conceptual components of the "Problem Solving DNA" core value at Company B are traced back to examples of original precedents in the data below, in Appendix O, Table 8.

The "Problem Solving DNA" core value was described and confirmed by study participants as the embodiment of an underlying belief that Company B succeeds in their specific industry niche because they are able to solve problems and deliver solutions where competitors typically fail. Company B succeeds through operationalizing their "Problem Solving DNA" core value through the systematic 
acquisition and grooming of employees with the potential, skills and proclivities of problem solvers.

\subsubsection{Company B, Core Value 3: A Culture of Integrity, Maturity \&} Respectfulness.

Study participants at Company B confirmed that their third core value, "A Culture of Integrity, Maturity \& Respectfulness," plays a critical role in maintaining the extremely high levels of competence and continuous personal growth of employees at Company B. The specific conceptual architecture of this core value, which resulted from application of the CCVI Technique, is captured in the bottom part of Figure 17.

The high priority placed on "Culture of Integrity, Maturity \& Respect" was formalized during the second Semi-structured Interview (Step 5 of the CCVI Technique) and validated as a core value in the final interviews and presentation. Appendix $\mathrm{O}$, Table 9 supports these results, and traces this core value and major conceptual components back to examples of original precedents in the data. This core value at Company B was described by multiple company leaders as contributing directly to human resource development. This value reflects a shared belief of the leadership team that the efficient growth and maturation of individuals' competencies require a social environment free from toxic or degrading influences. While the complete practical application of this value is elusive, the leadership team agrees that "A Culture of Integrity, Maturity \& Respect" enables the competence and personal growth related core values of "Problem Solving DNA" and "Do what you love." All text, concepts, 
pictures, relationships or associations presented here were validated by study participants during the final presentation.

\subsection{Introduction to Company C Case Study Results.}

The primary results discussed in this section are the unique core values identified at Company $\mathrm{C}$, which address the applied research question for Company $\mathrm{C}(A-R Q \# 1$ :

What are the unique core values at each subject company?). The unique core values at Company C include "The Company C Winning Mindset," "Caring for others," and "Balanced Analytical \& Emotional Intelligence" (See Figure 18 for a complete statement of Company C core values). 


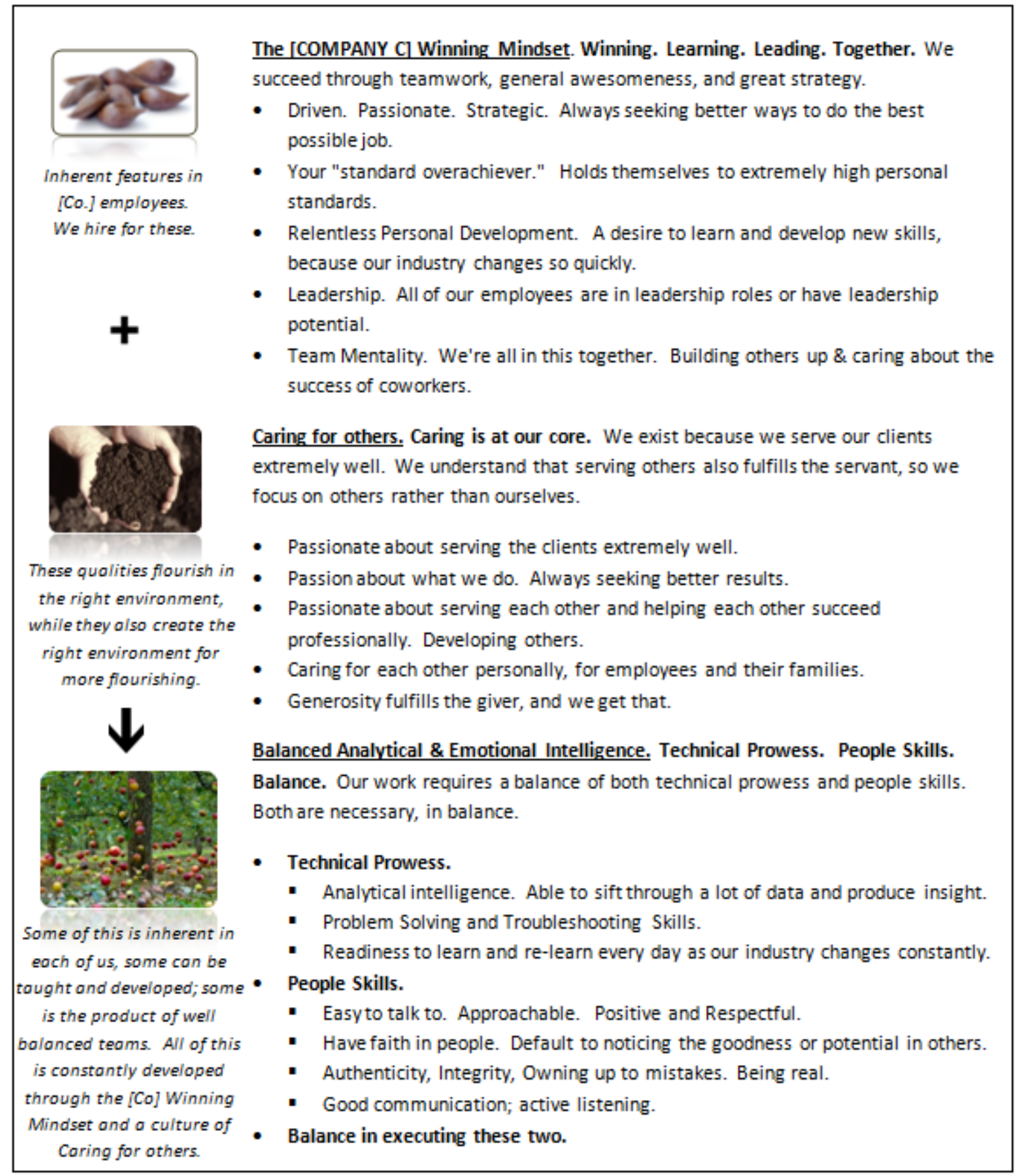

Figure 18: Company C Statement of Core Values. Figure includes an image of the full statement of core values, including associated behaviors and accompanying narrative identified through the applied CCVI Technique, as presented to and validated by study participants. The text and pictures displayed to the left of each core value are written and graphical representations of that narrative, validated by participants after the presentation. 
Core values are subject to a wide range of misinterpretation when taken alone, as with any similar statement of few words. To minimize the risk of misinterpretation, each core value is embedded within unique prose for clarifying the socially-shared meaning of each value for participants. Associated behaviors were also constructed from company data to accompany the brief core value statements to provide further clarity.

The second set of semi-structured interviews with Company C participants informed a cohesive narrative of how each value interrelates to the others. The narrative began to form as multiple company leaders independently described "Balanced Analytical \& Emotional Intelligence" as a cultural source of competitive advantage at Company $\mathrm{C}$, which was only possible through hiring and developing employees with "The [Company C] Winning Mindset," and fostered by the company's specific cultural environment which values "Caring for others." These discussions replaced the value ranking exercise at Company $C$, and led to further discussions of the interdependency between these three core values, including the holistic narrative illustrated through the pictures and descriptions to the left of the Company C core values shown in Figure 18. The text and pictures displayed to the left of each core value are written and graphical representations of that narrative, validated by study participants during the final presentation of results to Company $\mathrm{C}$.

Each Company C core value is presented in sections 5.3.1 - 5.3.3 below, along with a sampling of the data from which they were derived. Each Company C core value is traced back through major themes and concepts to its precedents in the data in 
Appendix 0 . While elements of these results may resemble other organizations, these results are not intended to be transferable because they are designed to reflect the unique shared language, symbols, and cultural realities of Company C.

\subsubsection{Company C, Core Value 1: The Company C Winning Mindset.}

The first core value, "The [Company C] Winning Mindset," is comprised of four major themes: Winning, Learning, Leading, and Together (see Figure 18). These themes, their constituent concepts and the associated behaviors together form what study participants call "The [Company C] Winning Mindset." The underlying belief of the study participants is that there is a company-specific mentality which is embraced and considered effective within the company. This specific conceptual architecture of this valued mindset, which resulted from application of the CCVI Technique, is captured in the top section of Figure 5.3.

This core value relates directly to the dominant "Clan" culture type of Company C, which was identified through use of the OCAl and verified through analysis of collected interview, observation and document review data. Company $\mathrm{C}$ participants hold the belief that highly developed human resources play a large role in their success. The Appendix $\mathrm{O}$, Table 10 traces this core value and major conceptual components back to examples of original precedents in the data.

The [Company C] Winning Mindset was independently described by multiple company leaders as 'inherent' or 'less teachable' features of employees or applicants to the company. These descriptions led to discussions of the interdependency of the core values, and the holistic narrative illustrated through the pictures and descriptions to the 
left of the Company $\mathrm{C}$ core values shown in Figure 18. All text, concepts, pictures, relationships or associations presented here were validated by study participants during the presentation.

\subsubsection{Company C, Core Value 2: Caring for others.}

The second core value at Company C, "Caring for others," is comprised of four major themes: Caring, Passion, Helping/Serving, and Generosity. These themes, their constituent concepts and the associated behaviors together form what study participants call "Caring for others." The underlying belief of the study participants is that there is a culture and shared mentality of serving and caring for others which is embraced and considered effective. This specific conceptual architecture of this valued mentality, which resulted from application of the CCVI Technique, is captured in the middle section of Figure 18.

This core value also relates directly to the dominant "Clan" culture type of

Company C. Classic hallmarks of Clan culture have been described as:

A very friendly place to work... It is like an extended family... Commitment is high... The organization emphasizes the long-term benefits of human resource development and attaches great importance to cohesion and morale... Success is defined in terms of sensitivity to customers and concern for people" (Cameron \& Quinn, 2011, p. 247).

Company $\mathrm{C}$ participants also hold the belief that highly developed human resources play a large role in their success. The caring environment, supported and appreciated by employees within Company $C$, is an environment that fosters human resource 
development. Appendix O, Table 11 traces this core value and major conceptual components back to examples of original precedents in the data.

The "Caring for others" core value was described and confirmed by study participants as both an impetus and as a result in and of itself. The basic concept is that the manifest beliefs, values and behaviors of employees which contribute to the shared experience of "caring for others" creates a positive feedback loop, whereby related beliefs, values and behaviors are increasingly encouraged and manifest as a result. These descriptions outlined during discussions of the interdependency of the core values, led to the holistic narrative illustrated through the pictures and descriptions to the left of the Company C core values shown in Figure 18, and the "caring for others"specific accompaniment to the left of the value in Figure 18. All text, concepts, pictures, relationships or associations presented here were validated by study participants during the presentation.

\subsubsection{Company C, Core Value 3: Balanced Analytical \& Emotional Intelligence.}

The third core value at Company C, "Balanced Analytical \& Emotional Intelligence," is comprised of three major themes: Technical Prowess, People Skills, and the Balance of those two. These themes, their constituent concepts and the associated behaviors together form what study participants call "Balanced Analytical \& Emotional Intelligence." The underlying belief of the study participants is that both analytical intelligence and the ability to interact and communicate skillfully with others are required for success at Company $C$, due in large part to the technical nature of the work, 
close collaboration with coworkers on client accounts, and direct contact between clients and technical account managers. So in practice, both analytical intelligence and "people skills" could be called company values; but striking a healthy balance between the two is considered absolutely vital to the success of the company. This missioncritical nature elevates the concept of balanced technical and people skills to the level of core value. The specific conceptual architecture of this core value, which resulted from application of the CCVI Technique, is captured in the bottom section of Figure 18.

This core value also relates directly to the dominant "Clan" culture and secondary "Adhocracy" culture of Company C. Company C participants hold the belief that both highly developed technical prowess in analyzing data, strategically managing accounts, communication and people skills all play a mission-critical role in employee and company success. Appendix O, Table 12 supports these results, and traces this core value and major conceptual components back to examples of original precedents in the data. 


\section{Chapter 6: Discussion}

This study developed a technique that extends the application of the Competing Values Framework to identify unique organizational core values, which was developed and empirically tested at three companies. This chapter is organized into two themes, focusing first on key considerations from the application and refinement of the CCVI Technique in sections 6.1 through 6.8 , followed by discussion of the contributions and limitations of this research, opportunities for future research and some closing remarks.

Sections 6.1 through 6.8 provide targeted topical discussions for each the eight steps in the CCVI Technique, which are described in detail in sections 4.1 through 4.8. The section number for each CCVI step discussion aligns with the section number in the chapter four presentation of that step in order to facilitate the readers ability to crossreference between the explanation of each step (in chapter four) and the relevant discussion material (in chapter six). Sections 6.1 through 6.8 also have a modular design, which is intended to maximize the ability of a reader to examine only sections of interest with minimal dependence on prior or subsequent discussion.

\subsection{Step 1: Initial Interviews}

Key considerations from the application and refinement of initial interviews are discussed in this section, including an analysis of the first interview question, the Core Value Assessment Exercise, and Organizational Culture Assessment Instrument (OCAI). This step, which involves conducting initial semi-structured interviews, is described in detail in Chapter 4.1 and in the Initial Interview Protocol (Appendix D), which includes a 
list of materials to bring, interview questions, and the base script for conducting these interviews.

Interview Question \#1. Interview Question \#1 of Initial Semi-Structured Interviews, "Tell me, what is one piece of advice you would give to a new employee or applicant about how to thrive at this company?" is designed to encourage interviewees to reflect on what the company values in a simple, concrete way and immediately begin to identify the top, most important values. Interview Question 1 is carefully deconstructed in Table 3 below because the discussion resulting from this question provides some of the richest material for data mining in the CCVI process.

\begin{tabular}{|l|l|}
\hline \multicolumn{2}{|l|}{ Interview Question Subtle Features } \\
\hline Question Text & Purpose and/or Subtle Feature \\
\hline "Tell me," & $\begin{array}{l}\text { A linguistic device that primes the listener to share or } \\
\text { divulge information }\end{array}$ \\
\hline $\begin{array}{l}\text { The explicit focus in this question on "one piece" of advice } \\
\text { primes the listener to intuitively identify the top, most } \\
\text { advice" }\end{array}$ \\
$\begin{array}{l}\text { important response. In context, this primes the } \\
\text { interviewee to divulge a response that has a high } \\
\text { likelihood of relating directly to their top perceived core } \\
\text { value at the company. }\end{array}$ \\
\hline $\begin{array}{l}\text { you would give to a } \\
\text { new employee or } \\
\text { applicant" }\end{array}$ & $\begin{array}{l}\text { Primes the listener to reflect on a tangible concept - } \\
\text { advice - rather than an abstract concept. Starting with } \\
\text { concrete concepts makes it easier for most interviewees } \\
\text { to digest the question and participate actively in the } \\
\text { process. }\end{array}$ \\
\hline $\begin{array}{l}\text { This question asks the interviewee to reflect on how best } \\
\text { applicant" }\end{array}$ & $\begin{array}{l}\text { to describe their 'advice' to a newcomer, or someone with } \\
\text { little inside-perspective on the company. This is intended } \\
\text { to improve the ability of the interviewer (an outsider) to } \\
\text { grasp the spoken response of the interviewee. }\end{array}$ \\
\hline $\begin{array}{l}\text { "advice... about how to } \\
\text { thrive at this } \\
\text { company?" }\end{array}$ & $\begin{array}{l}\text { most important, success-shaping features of the company } \\
\text { from an employee perspective. These responses typically } \\
\text { reflect or relate to what are most valued - or top values - }\end{array}$ \\
\hline
\end{tabular}


at the company.

Table 3: Initial Semi-Structured Interview Question 1 Discussion Table. Interview Question 1 carefully deconstructed in this table because the discussion resulting from this question provides some of the richest material for data mining in the CCVI process.

An important feature of the CCVI Technique is beginning inquiries with concrete and factual data collection and inquiries, and to progress into increasingly abstract and theoretical concepts from those straightforward beginnings. Asking directly about company values too early in the process carries a higher risk of encountering espousedvalues (the values that are talked about) rather than values-in-use (the values upon which decisions and actions are based).

An important caveat is that sometimes interviewees may respond with less valuable or basic competence related advice rather than concepts with high-potential to reflect core values. Success in semi-structured interviews depends in part on the skill of the interviewer to probe or mine appropriately for the information that is needed.

During the process it was also observed that the first response(s) were usually considered of higher importance than the residual data mined by probing, likely resulting from the explicit focus in this question on "one piece" of advice.

Core Values Assessment Exercise. In order to reduce ambiguity during data analysis, the initial interview process was improved by changing the protocol of probing for "qualities" during the Core Values Assessment Exercise to probing directly for "Values or Behaviors." The improved language is reflected in the Initial Interview Protocol (Appendix D). Early iterations of the Core Value Assessment Exercise also treated all three employee groups equally, but focus was shifted primarily to the "star 
employees" probe to improve data quality. The underlying rationale of this shift in focus is that identifying the shared values and behaviors of star employees are more reflective of shared organizational core values than the other two groupings. This realization is supported by the findings of Lencioni (2012, p. 102) who wrote:

One of the best ways to go about identifying an organization's core values is to [first] identify the employees in the organization who already embody what is best about the company and to dissect them, answering what is true about those people that makes them so admired by the leadership team. Those qualities form the initial pool of potential core values.

The values and behaviors resulting from the "leadership team" probe were commonly described by participants as having displayed the values and behaviors of "star employees" over long periods of time, in addition to their unique values and behaviors that were not shared at the company level of analysis. The values and behaviors resulting from the "not-so-hot" probe were commonly described as the antithesis of "star" employees. This is appropriate as the not-so-hot category functions as a counterfactual probe, mining for an opposite situation which served to clarify the Star category. The not-so-hot category was most useful at Company B in serving to clarify the "Problem Solving DNA" core value. The ability for the researcher - an outsider - to grasp the socially shared meaning of "Problem Solving DNA" was improved by descriptions such as "afraid to fail or make mistakes," "gets hung up too easily," and "needs step by step instruction. In summary, discussion resulting from the "star" employee probe provided the most direct value to the goal of identifying core value candidates during the core values assessment exercise, while the "leadership team" and 
"not-so-hot" provided useful supplemental or contrasting details that enriched the overall data.

Organizational Culture Assessment Instrument (OCAI). The CCVI Technique employs the standard CVF-based Organizational Culture Assessment Instrument (OCAI) during initial interviews. Application of the CCVI process uncovered that the accuracy of the OCAI (when used alone to assess organizational culture) may be overly susceptible to differences in interpretation. Participants may interpret the standardized language used by the OCAI very differently, which can then influence their point allocations. For example, at Company B, the "Market" language was interpreted very differently by participants. Participant 01 and 03 both indicated a strong preference for a decrease in the Market quadrant for very different reasons. Follow up probes during second round interviews uncovered that Participant 01 preferred a decreased Market profile because he associated the CVF market descriptions with a "profit-driven mentality," which he strongly disliked and incidentally associated with Participant 03 (amongst other employees). Participant 03 also preferred a decrease in the "Market" archetype for the company future, but explained that the focus of this preference was on the "hard driving" aspects of OCAI Market descriptions which incidentally were embodied by Participant 01.

In this case, two individuals representing two distinct cultural factions both interpreted the "other" faction to be the embodiment of the "market" archetype, and recorded a preference for the same OCAI change, while the desired changes they 
intended for the assessments to reflect were exactly the opposite. Unprobed OCAI results in this case would have created the false impression of agreement where there was in fact sharp contrast. However, these differences were probed and verified in second semi-structured interviews, illuminating the value of the CCVI Technique's deeper inquiry into OCAI results when assessing a culture, and points to possible improvements in the codification of the OCAl archetypes.

The OCAI may also be susceptible to influence from short-term changes in organizations, as participants may fail to distinguish between culture and shorter-term situations or changes without prompting. If significant changes are obvious or underway in an organization, such as an unexpected busy season or recent change in leadership, the practitioner should emphasize for participants that the goal with the OCAl is to assess the longer-term nature of culture (as opposed to shorter-term changes) to yield more accurate results. This susceptibility became apparent at Company A when large discrepancies between the OCAI results of participants were probed during second round interviews. OCAI results for Company $A$ also failed to produce an accurate assessment of the dominant culture type (see Figure 19, below). 


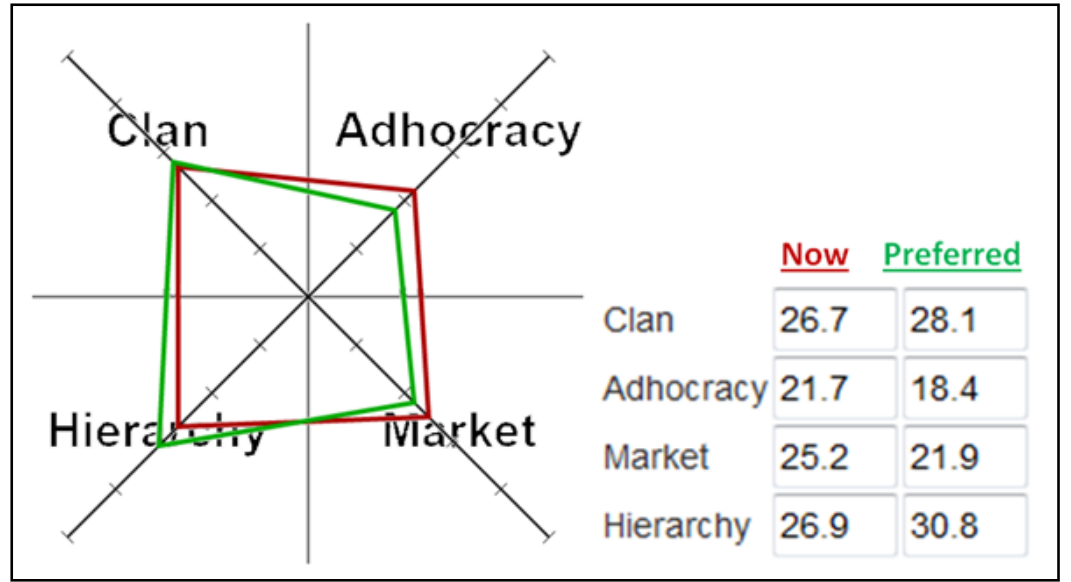

Figure 19: Company A Combined OCAl results. Figure shows a lack of clear dominant culture type based on OCAI results. The dominance of a Market culture was identified through review of all data collected at Company $A$, and later validated by company leadership.

The correct dominant culture type at Company A was identified as Market through application of the CCVI Technique, and was subsequently validated by company leadership during the presentation.

In summary, the synthesis of qualitative data and standard OCAI results in this research surpassed the accuracy of the OCAI alone. This outcome suggests that the CCVI Technique may improve the practical application of the Competing Values Framework in assessing organizational culture.

\subsection{Step 2: Participant Observation}

Key considerations from the application and refinement of Participant Observation are discussed in this section, including the introduction to participants, establishing rapport, the type of participant observation, and optional key informant interviews. This step (conducting Participant Observation) is described in Chapter 4.2 and in the Participant Observation Field Guide (Appendix H), which includes a list of 
materials to bring, interview questions, and the base script for conducting these interviews.

Introduction to Participants \& Establishing Rapport. Company leadership must announce or introduce the researcher before beginning participant observation in order to obtain informed consent and prime employee expectations. It was found that the quality of this introduction greatly affects the quality and usefulness of participant observation and resulting field notes. Company A leadership gave a straightforward, matter-of-fact introduction. In response, the researcher was cordially treated as an outsider by most employees, which in turn limited his ability to become immersed in the field and develop rapport with employees. These findings support the notion that in order to obtain quality field data it is important to establish rapport with or be accepted into the community being studied (Hume \& Mulcock, 2013). The importance of the introduction in establishing rapport and obtaining quality data from participant observation was became clear at Company B, where the leadership gave a warm and lively introduction, encouraging employees to feel comfortable sharing their perspectives voluntarily with the researcher. The quality and usefulness of participant observation and resulting field notes were considerably higher at Company B. The following reflective memo captured in field notes reflects the initial response of the researcher:

[Employee 04] took me for a tour of the company on Tuesday (first day of PO), introducing me to everyone individually. He announced my presence and project at the morning meeting, before I was allowed to arrive. There was a 
client visiting that morning who, for confidentiality reasons, prevented my being present. As [Employee 04] introduced me to employees one after another, he would often begin with "I'd like you to meet Anthony; this is the guy I warned you about this morning." Folks usually laughed or smiled as they shook my hand, appearing at ease with [Employee 04] and my presence. I was told later by another employee that [Employee 04] gave me a very good intro, asking folks to share with me anything they wanted for my research. [Employee 02] said "he gave you a very nice introduction. I think folks will want to share things with you as long as they know it's anonymous." I found the employees to be quite friendly and quick to volunteer stories and information they thought would help my project along. I was a bit shocked by how - especially the very first person I approached without [Employee 04]'s escort (an electrical engineer) - just heaped his perspective on me. I thanked him for sharing his perspectives with me so freely and he replied that "it wouldn't be very nice to stonewall or sandbag a guy trying to finish his school project." I appreciate that!

This memo also highlights the concept that employees who are interested but hesitant to volunteer information with the researcher sometimes need to be reminded that anything they share will remain confidential.

Type of Participant Observation. After multiple employees generously volunteered their perspective on Company B culture and values in the wake of the quality introduction I was given by company leadership, I wrote this memo: "I was amazed with how much he volunteered so much of what I would love to hear for the sake of my project. The intro the management here gave for me (and perhaps the openness of the employees) exceeds that of the previous company by orders of magnitude." This observation led to a refinement of the CCVI Technique: taking care to request a good quality introduction from company leadership, as described in Chapter 4.2 (CCVI Step 2: Observation). This improvement in the CCVI Technique can be understood as precipitating a change in the level of Participant Observation along the 
spectrum of engagement described in existing literature, which ranges from NonParticipatory to Complete Participation (Spradley \& Baker, 1980). At Company A, "Passive Participation" was employed, where the researcher remains a bystander or outsider, and has a limited ability to establish rapport with the target population or gain insight through immersion (DeWalt \& DeWalt, 2010). Higher quality data were collected at subsequent companies through "Moderate Participation," where the researcher takes a balanced role between "outsider" and "insider." This moderate participation enables achieving the balance of the detachment necessary for objectivity, as well as the level of rapport and immersion necessary for obtaining an in-depth understanding of the field, and gaining insights the subject population may share with the researcher (Gair, 2012).

Establishing rapport is critical to achieving moderate participation during field research. There is invariably a time span at the beginning of observations where expectations and relationship are norming, and participants are most curious about the researcher. It is critical in this early phase for the researcher to be friendly and unobtrusive to accelerate the process of employees becoming comfortable enough with the new presence to ultimately reduce the impact of researcher presence on participants so they can manifest normal work behaviors. The tendency for participants to put up a façade or otherwise change their behavior based on the presence of a field researcher is known as the observer expectancy bias (Abernethy, 2015). The observerexpectancy effect was also mitigated in this research by the use of member-checking 
and triangulation, two classically employed tactics for employing rigor in qualitative research (Carter et al., 2014).

(Optional) Key Informant Interviews. Key Informant Interviews were employed to supplement Participant Observation at Company $\mathrm{C}$, which proved to be the most challenging of the three companies included this research. Much of the work at Company A and Company B included physical labor or manufacturing product. Company $\mathrm{C}$ was an online marketing management company and the work of the operations team is almost entirely done mentally (analyzing trends) and in unfamiliar computer interfaces. The following reflective memo written during Participant Observation captures the researcher's first experiences of this difficulty:

The larger table is quieter, and it's unclear who is doing what work, how much, or how often, because they are all at their desks, looking at their computers, with the screens flickering between various things... Youtube, facebook, videos, games... Whether it's potentially related to clients or just recreational, it's unclear to me as an observer. One thing is clear though. Google Analytics and other spreadsheets are often up on at least one screen, and data is viewed regarding view rates, click through rates, etc. An observer can tell that the screens are up, but it's unclear what they're doing, if they're working, or thinking, or not... it's difficult for me to observe the processes effectively since they are using mediums/interfaces that I am unfamiliar with.

In response to this difficulty, two of the most influential leaders from the operations team were identified and asked to participate in an initial semi-structured interview. Informed consent was obtained, both from company leaders allowing their participation and from the two key informants. These initial semi-structured key informant interviews occurred near the end of participant observation and utilized the same materials, questions and probes as CCVI Step 1 (Initial Semi-Structured 
Interviews). Three differentiating features of these interviews were that the time was used to: a) verify and member-check observations; b) probe extensively into the work processes, relationships, and trends that were difficult to observe due to the nature of the work; and, c) gain insights and test initial hypotheses regarding the concepts and themes emerging from the initial coding and analysis that had been done up to that point. This early phase of gathering feedback on initial hypotheses in particular added significantly to both the quality of the CCVI results, and the quality of insights and suggestions developed for guiding organizational change delivered in the final presentation. The improved quality of initial hypotheses and suggestions became evident in all subsequent interviews with company leaders, as indicated by the following comment from [Employee 03] at Company B in response to a hypothesis shared during a second round interview:

That's very insightful for you to link those two together because I think that you are exactly on the right track there. Yeah. Just for the amount of exposure you've had to our company, to be able to connect those dots; you're very good at what you do.

In summary, including key informant interviews in the process is a viable option that can improve the quality of collected data and provide an opportunity to probe more deeply for insights that can improve the suggestions made to guide organizational change during the final presentation.

\subsection{Step 3: Document Review}

The three target companies included in this study were small ( $<55$ employees), privately owned firms with a limited degree of formal documentation. Some informal 
documentation reviewed at subject companies exceeds the scope of this work, such as marketing materials at Company $\mathrm{A}$, or statistics regarding employee tenure and turnover at Company B (which factored into suggestions for guiding organizational change). The major items reviewed at target companies included Performance Evaluations, Employee Handbooks, and Pre-existing Values Statements.

Performance Evaluations. The one document type included for review at all three subject companies was Performance Evaluations for employees. The CCVI Technique is designed to identify behaviors associated with company core values, which in turn allows those specific behaviors to be easily included in Performance Evaluations as line items. Associated behaviors are also included in Core Values statements produced by the applied CCVI Technique to increase the alignment of these two formal documents. The suggestion to include the behaviors associated with company core values as line items in Performance Evaluations was made to all three subject companies during the presentations.

Employee Handbooks. While all three companies had Employee Handbooks in which company values could have been articulated, the utility of those documents was variable. Company A cordially denied the researcher access to the Employee Handbook, citing with ostensible honesty that the document was embarrassingly out of touch with reality, and that it would need to be updated significantly before it could provide any real value to the investigation. Reflection on the subsequent review of Company $B$ and C Employee Handbooks led to realization that at small companies documents are very likely to be out of touch with current cultural reality than are the other two forms of 
primary data collection (interviews and participant observation). In response to this realization, the bulk of the research focus when drafting Core Values was placed on data from interviews and on field notes from observation. The lessons learned from these experiences were subsequently incorporated into the formal CCVI Technique methodology, as described in sections 3.3 and 4.3 of the present research.

Effective handling of Pre-existing Values statements. Two of the three target companies included in the research had some form of pre-existing values statement. Company A had a formal, pre-existing "Core Values" statement posted in auspicious places, and Company B had two formal statements of values that were used in different but complementary ways. One was a seven-paragraph letter from the owner reflecting on company values; the other a matrix intended to clarify the company priorities along two continuums: (1) Customer > Employee > Business; and, (2) Safety > Product Quality $>$ Service $>$ Cost. Language and values in pre-existing values statements were treated as a secondary stock of language and concepts for telling the tale of the core values uncovered by interview and observation in this research for two reasons: (1) because the contents of the formal documents available for review at the small participating companies were noticeably out of touch with company culture when compared to interview and observation data; and, (2) because the goal of the applied CCVI Technique in this research was to provide a fresh assessment of company core values, which may which may validate, replace or fall short of prior statements.

Attention was also given to distinguishing between core and other types of values in the second round of interviews and the final presentation. Company A, for 
example, included "Safety" as a Core Value in their pre-existing statement. Care was taken to address the reclassification of Safety as a permission-to-play value in second round interviews, and again in the final presentation to the company. While the importance of safety was at no point underplayed, the formal distinction between core values and "other important values," including safety, was unanimously embraced by company leadership. To further illustrate how this distinction was handled, an example using data from Company $A$ is discussed below.

Example from Company A: handling preexisting values statements. Company A had a preexisting values statement (See Figure 20, below) which informed and impacted the language, content, and formatting of the final results significantly.

Integrity
Honesty in all we do. Whole and complete systems.
Relationships
We value strong relationships in all areas; with employees,
clients (internal and external), and our vendors.
Respect
Treat each other with dignity and respect while expecting others to do the same.
Results
Measured progress. We strive to achieve excellent results.
We accept personal responsibility and accountability.
Safety

Figure 20: Company A Preexisting Statement of Core Values. Figure includes the published, pre-existing values at Company A, called "core values." 
Application of the CCVI Technique yielded a separate set of Core Values, and a new statement was crafted in response. The new Company A values statement (see Figure 21, below) includes six total Company A values cast in simple language at the request of Company $A$ leadership, includes behaviors that serve to increase comprehension and integrate with performance reviews, and distinguishes between the three identified core values (in grey box) and other important values identified at Company A. 
New Values Company A (core values in grey)

\section{Delivering Results}

We strive to achieve excellent results.

- Bringing a positive, can-do attitude to every challenge is the key to our success.

- We accept personal responsibility and accountability.

- We work hard \& smart.

\section{Integrity}

Honesty in all we do.

- We must always walk the talk, own our actions and take responsibility.

- Do work to the best of your ability and to the highest quality.

- We don't take shortcuts. We do it the right way.

\section{Developing our People}

We are all craftsmen, mentors and teammates. We helpeach other improve every day. We do this because we honestly care about everyone's personal growth and it makes us more effective.

- Be willing to listen and learn.

- Help and train each other.

- Ask questions.

\section{Respect}

We are experts and we are professionals. We treat each other with dignity and respect while expecting others to do the same.

- Be sensitive to others, be patient with people whodon't know as much as you

- Be accountable for the impact of your actions on others.

\section{Safety First!}

Work free from harm and communicate issues. We care about our customers' safety too!

- Build safety into everything we do.

- Be a role model \& hold each other accountable for safety.

- Follow safety rules. Speak up if you see an unsafe act.

- You can be fired for a safety violation.

\section{Focus on the Long-term}

We aspire to be a career company. We value loyalty, trust and commitment with each other, clients and vendors. Working with the same people every day creates efficiency. At [Company], relationships, caring and commitment can create that stability for everyone.

- Do work and build relationshipsthat will last.

- Show long-term commitment. We're here for careers, not jobs.

- Work as a team, leveraging individual strengths and overcoming differences.

Figure 21: Company A Complete Values Statement. Figure includes a snapshot of the top six, most important values at Company $A$, including and distinguishing between the three identified core values (in grey box) and other important values. 
While this result exceeds the technical scope of the CCVI Technique (by including three non-core values in addition to the identified core values), it also served the practical purpose of improving the usefulness of the final results for company leadership. Note that the language and concepts included in the core values resulting from the applied CCVI Technique at Company A (Figure 21) resemble the preexisting statement of core values at Company A (Figure 20). This consistency is for two primary reasons: to increase the ease of transition from the old statement to the new; and, because there were elements of the preexisting statement that were valid. However, several key distinctions between the results of the applied CCVI Technique and preexisting core values statement were made and communicated during presentations. These distinctions include:

- "Developing our People" was identified as a core value that was missing from the preexisting statement.

- Core values were distinguished from other important types of organizational values (e.g., "Respect" and "Safety" were reclassified as a Permission-to-Play values), and non-core values were excluded from the resulting core values statement.

- The verified and validated hierarchy of core values (ranked in order of importance) was established through analysis of the ranking/weighting exercise from Interview 2.

- Associated behaviors were identified and included through the applied CCVI Technique to improve ease of comprehension and practical application of the results (e.g., associated behaviors can be easily incorporated into performance review forms).

- A cohesive narrative emerged from the relationship between the three core values identified, which provided clear guidance for organizational change.

\subsection{Step 4: Initial Data Analysis}


The overall goal of the Initial Data Analysis is the coding and analysis of all collected data in order to create the culture profile and core value candidates used in the second set of semi-structured interviews with company leaders. Key considerations from the processes of preparing culture profiles and core value candidates are discussed in this section.

Creating Culture Profiles. Culture profiles based on OCAI results at subject companies are described in detail in Chapter 4.4. During the second round of interviews, discussion of the Overall Culture Assessment forms (Appendix J) and Itemized Culture Assessment (Appendix K) provide the greatest opportunity to introduce and sensitize interviewees to the Competing Values Framework (CVF). Together with the cultural profile results from the OCAI, the theoretical framework of the CVF provides the crucial structure and language needed to foster deep and precise inquiry into the often nebulous topic of organizational culture. Put another way, the OCAl-based culture profiles and associated discussion allow for a depth and precision of inquiry into organizational culture and core values in the second interview that would not be possible in the first interviews or without a theoretical framework comparable to the CVF.

The subtle intention behind the Overall Culture Assessment form (See Appendix J), as evidenced by the Competing Values Framework twice rendered on that worksheet, is to sensitize the interviewee as much as possible to the language and structure of the framework. The two depictions of the CVF also follow two styles (one written in a block of descriptive text, the other organized by key features) to facilitate comprehension 
regardless of information processing tendencies of interviewees (See Figure 22 for

example, below).

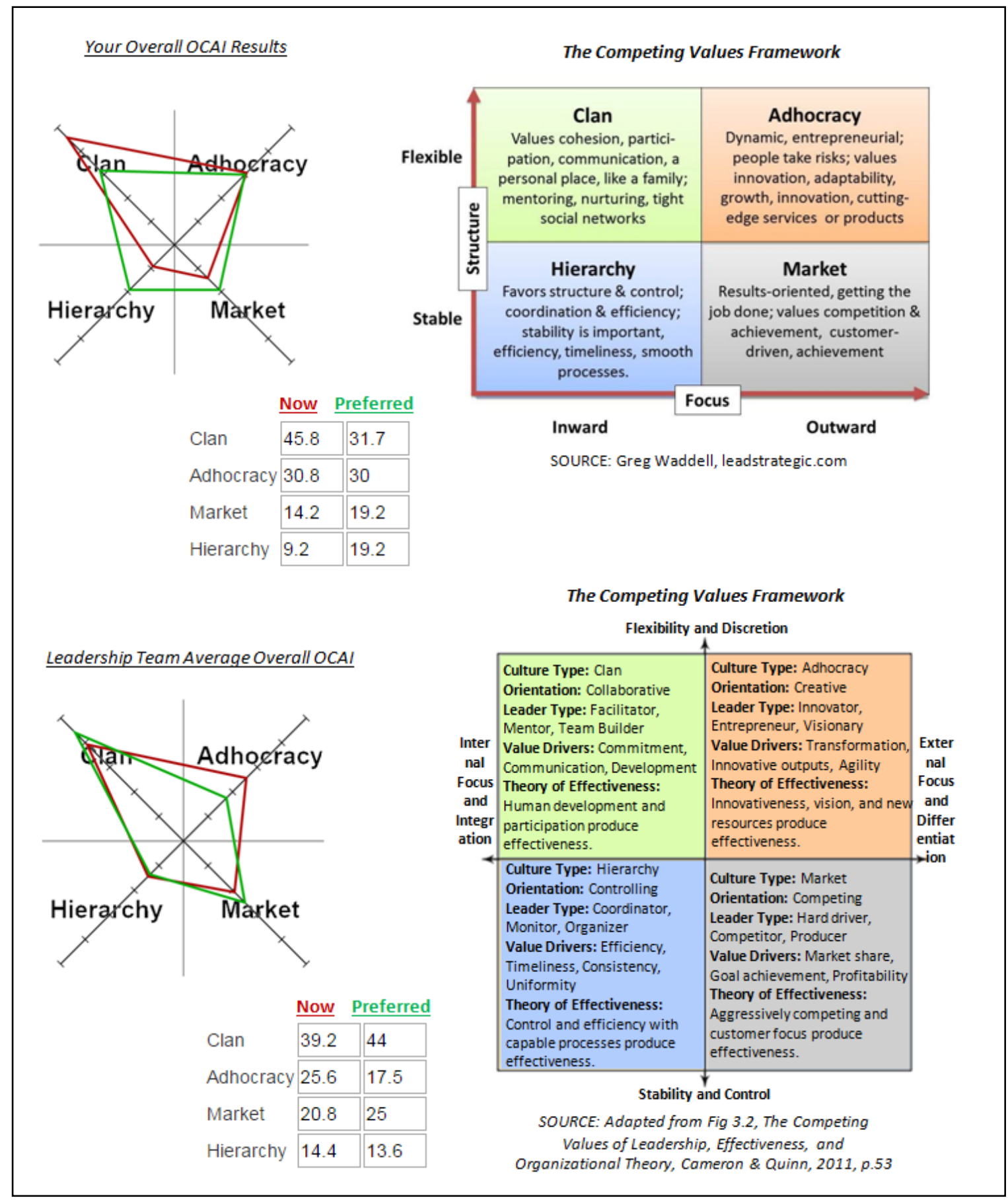

Figure 22: Snapshot of a complete Overall Culture Assessment form. See Appendix J for template and full-sized text. 
The Itemized Culture Assessment form (See Appendix K, or Figure 23 for example, below) also contains the full text of each description of the CVF quadrants associated with each question in the OCAl, allowing interviewees to reflect at will on the language and concepts of the CVF while reviewing and discussing OCAI results. 


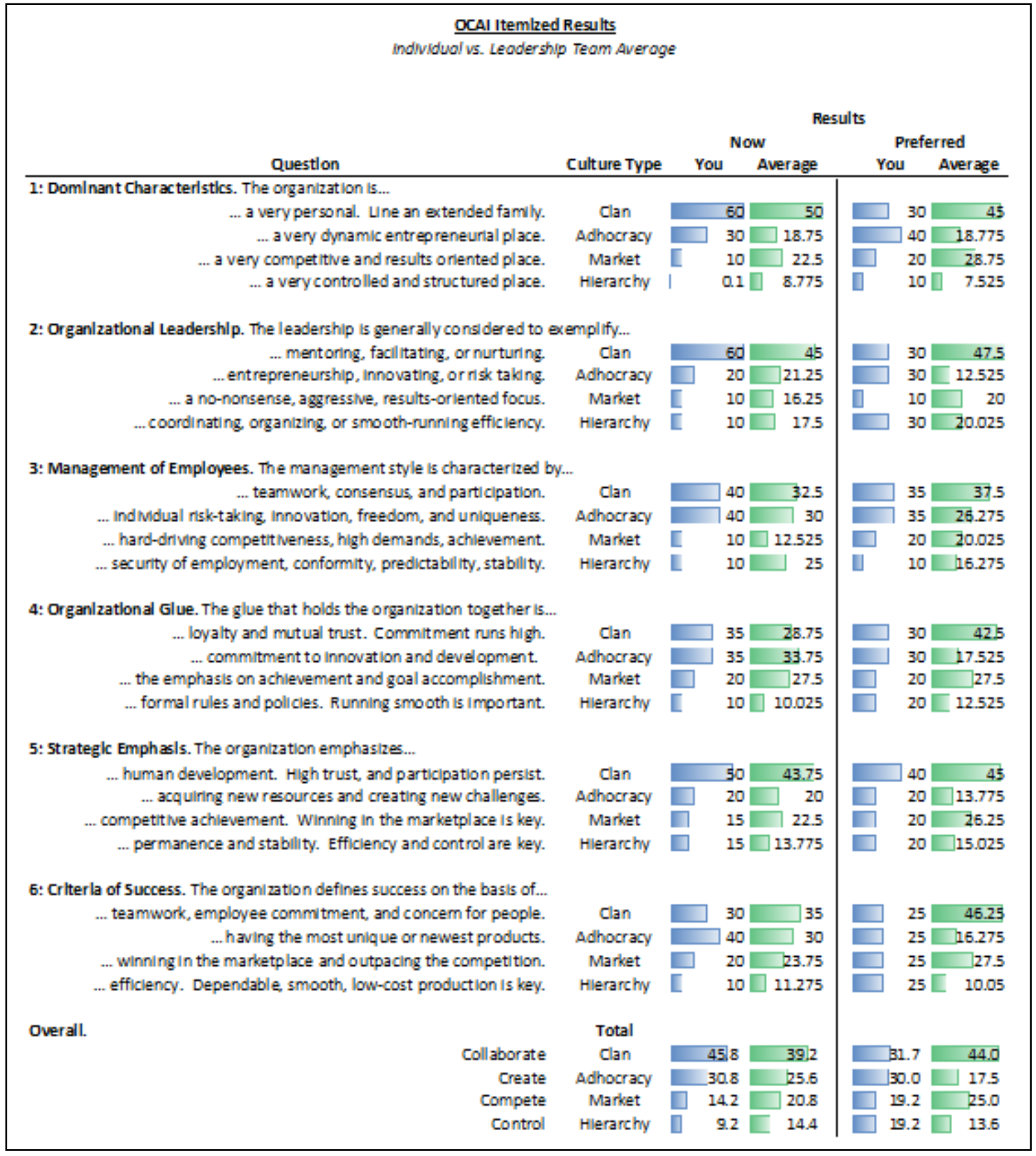

Figure 23: Snapshot of a complete Itemized Culture Assessment form. See Appendix K for template and full-sized text.

Use of color-coded data bars was incorporated into the Itemized Results

template during CCVI application and refinement to improve readability and

comprehension of this extensive worksheet. In this research, the Itemized Results for 
each participant were carefully reviewed before their second interview to remind the researcher of the specific results and questions that called for more nuanced probing. Because the OCAI is easy to administer and requires little time or effort to complete, administering the assessment to a wider range of organizational members is also a viable option. It was found at Company $\mathrm{C}$ that increased use of the OCAI allows for increased comparative analysis across organizational groups. This was suggested by leadership at Company $B$, which led to the subsequent request and granting of additional IRB permissions in this research. When offered, Company $\mathrm{C}$ was also interested in this optional service, and company-wide OCAls were distributed. A total of 15 out of 18 employees completed the assessment, and results were aggregated and displayed in four groups, as shown in Figure 24, below. 
125

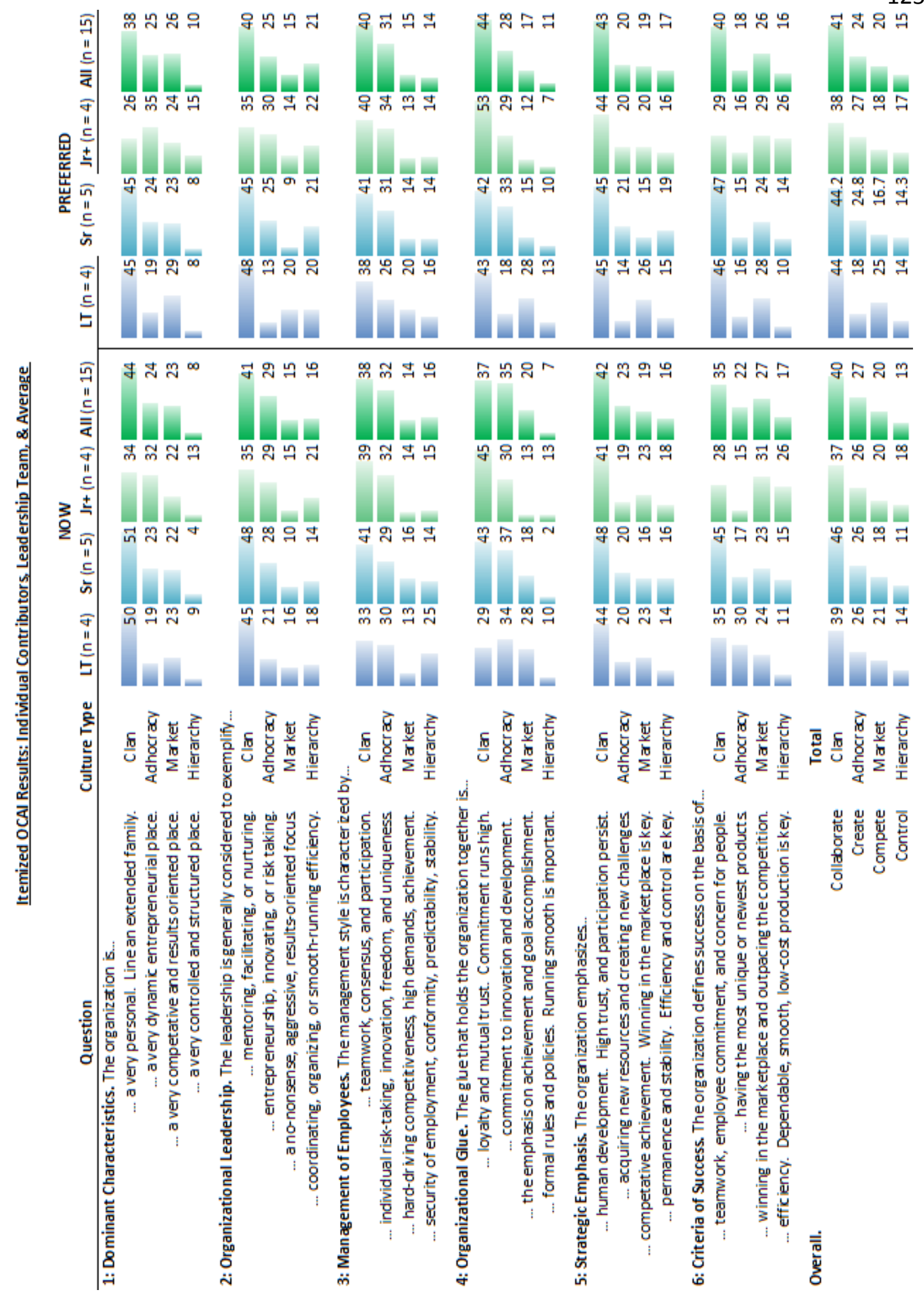

Figure 24: Snapshot of a complete Company-Wide Culture Assessment form. Full-sized template also shown in Appendix $K$. 
The groupings employed in this additional OCAI application included the leadership team, senior account managers, junior account managers, and all employees. While analysis of the additional OCAI data and resulting discussion did not significantly impact the assessment of core values at Company $C$, valuable insights and suggestions were mined for guiding organizational change at the company. This optional companywide OCAI Results Template used in the second round of interviews at Company $\mathrm{C}$ can be found in Appendix $\mathrm{K}$.

Further Discussion of Coding and Initial Analysis. At Company A, interview transcripts and field notes were coded by hand on printed pages. However, subsequent manipulation or analysis of the hand-coded data proved cumbersome and created extra work when it came time to formalize and organize the codebook. A word processer software was later used to tag transcribed data (with code names), and a macro built to export both codes and associated data to a spreadsheet for further analysis.

The code names used to identify data included signals about the original context or location of the data in order to assist the researcher in maintaining contextual understanding of all data even when iteratively reorganizing codes during thematic content analysis. For example, coded data that resulted from the "Star" prompt of the exercise in initial interviews might be given the code name, "Problem Solving = Star," and responses to the first interview question were also marked with a "Q1" in the code name.

A subtle but important aspect of effective coding also includes the use of gerunds to increase the mental focus and engagement of the researcher (B. G. Glaser, 
1978). An example from the present research is the code, "Problem Solving = Star," which reveals an instance of "problem solving" being associated with "star employees" in data collected at Company B. Charmaz supports Glaser's guidelines and stresses the use of gerunds in coding, pointing out that "adopting gerunds fosters theoretical sensitivity because these words nudge us out of static topics and into enacted processes" (Charmaz, 2006, p. 136). As the collected data at Company A was coded during initial analysis, patterns in the data began to emerge naturally as similar concepts were encountered, which prompted the following reflective memo:

The Codebook's Self-Organizing Process Memo: As I coded each question, response, story and concept, I constantly reflected on how the data fit together, allowing the organizational tree of the codes to morph organically as new codes emerged. This was much simpler than trying to force a framework would have been - it came very naturally. The method that worked itself out was to start by scrawling emerging concepts (obvious code families) onto a blank page as I worked through the coding, and found that they layered naturally into categories as well. As the organizational trees changed and grew, I periodically typed up the most current forms for ease of reading and amendment. I would then scribble in changes and additions as they arose, allowing the initially-ambiguous form of the data to unfold almost effortlessly as I focused on coding. It is likely that my high tolerance for ambiguity made this process extremely simple. It was delightful to watch the organizing happen - as though the data did in fact structure itself.

The above memo reflects an intuitive application of the 'constant comparative method' (B. Glaser \& Strauss, 1967) as the researcher moved from codes to thematic content analysis - identifying themes and patterns in the data. Strauss and Corbin (2014) also present an intermediate type of coding known as axial coding, which requires the specification of properties and dimensions of categories in order to 
facilitate the integration of relevant categories. However, grounded theorists do not universally embrace axial coding as a necessary step. Charmaz, a leading expert in grounded theory analysis, states that:

Axial coding provides a frame for researchers to apply. The frame may extend or limit your vision, depending on your subject matter and ability to tolerate ambiguity. Students who prefer to work with a preset structure will welcome having a frame. Those who prefer simple, flexible guidelines-and can tolerate ambiguity-do not need to do axial coding. They can follow the leads that they define in their empirical materials. (Charmaz, 2006, p. 61)

The dominant method for conceptually organizing the data during initial analysis in this research was an iterative use of clustering. Clustering is a flexible information processing technique where material - codes in this case - is grouped into visual clusters, producing a non-linear, tentative, and easily alterable map of the work (Rico, 1983). Clusters of similar codes became treated as concepts, and clusters of concepts became themes or categories (see Figure 25, below).

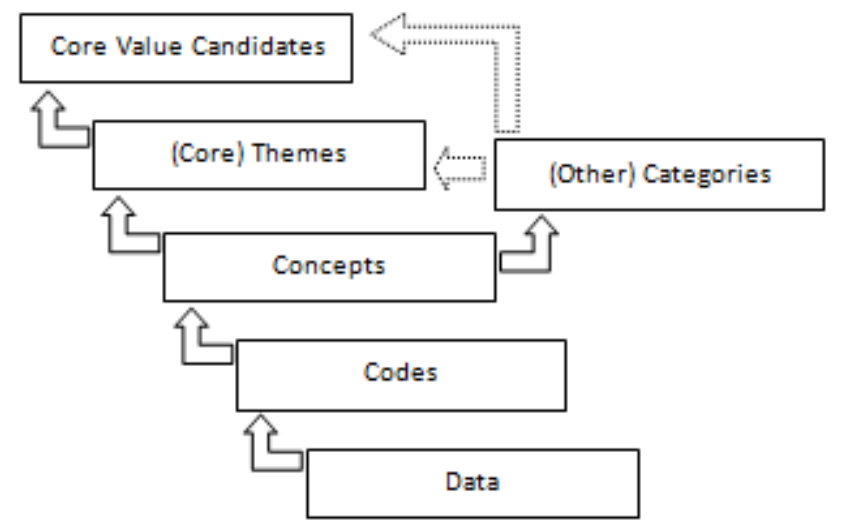

Figure 25: The Typical Conceptual Mapping from Data to Core Value Candidates in this research. Core Themes and (Other) Categories are treated separately in this figure to illustrate the divergence between major and minor trends in the data - trends that may or may not contribute to identification of Core values. 
Categories can be major or minor, and sometimes irrelevant, based on the richness of the data. At Company A, for example, tremendous effort was put into detailing categories and trends in the data that would never become core value candidates. Exhaustive lists of perceived organizational values were detailed (such as permission-to-play, field-specific and office-specific employee values) regardless of their limited relevance to organizational values. Upon completion of Company A analysis, the over-analysis of lower quality values was determined to be scope creep and the scope of work in subsequent company analyses was improved. The critical question to answer in assessing categories is simply which should be elevated to themes or core value candidates. The answer to this question is that the researcher should "raise the categories that render the data most effectively... that make the data meaningful and carry the analysis forward" (Charmaz, 2006, p. 139).

In this research, analysis and clustering were highly iterative, involving multiple drafts and a willingness to start afresh when drafts were not producing the desired clarity or insight. As dominant themes emerged as potential candidates for core values, formal "core values candidates" were carefully constructed from the language and morphologies of component data clusters and included in the Core Values Identification form (Appendix M) for use in the second round of interviews. Behaviors articulated in the data of associated clusters were also included in the Value-Behavior Association form (Appendix $\mathrm{N}$ ) for use in the second round of interviews.

\subsection{Step 5: Second Interviews}


The second round of semi-structured interviews provides precise and especially rich data for developing the emerging form of the researcher's theory of organizational core values at a target company. These interviews were engineered and evolved with exactly that aim, fulfilling a role in the grounded theory process known as Theoretical Sampling. While initial data collection provided several conceptual candidates that could possibly be included in the final theory, theoretical sampling provides the researcher with specific directions on the precise contents or organization of the final theory (Charmaz, 2006, p. 100). In the CCVI Technique specifically, initial data collection provided several core value candidates that could possibly be included in the final statement of organizational core values, and theoretical sampling in the second round of interviews provides the researcher with specific directions on the precise contents and organization of the final core values statement. The final process for completing this second round of semi-structured interviews with company leaders is described in great detail in Chapter 4.5. The Chapter 4 treatment precludes the need for further processoriented discussion. However, this step in the CCVI Technique was perhaps the most improved by iterative applications, and several key considerations from those refinements will be discussed in this section.

Moving Quickly through OCAI Data. Second round interviews went long at Company A because there was so much material to cover. This was due in part to an overly meticulous presentation of OCAI results, where nearly every set of results on the Itemized Culture Assessment page was described and discussed, line by line. While a 
researcher should take the time personally to sift carefully through the data, only the most significant trends and features of OCAI results should be brought to the attention of interviewees for probing or clarification. To assist with this narrowing of scope and focus, prompts were added to the second interview protocol to:

- Let interviewees know that "we have a lot of material to cover so we are going to move quickly."

- Avoid information overload for interviewees by letting them know that they "don't need to fully grasp everything."

- Focus the discussion on the key trends with interviewees while reviewing the OCAl (which serves not only to save time, but also to maintain higher levels of engagement and interest from interviewees)

- Let interviewees know that they are welcome to keep the printed OCAl results on the culture assessment worksheets. Some will be eager to review the itemized results at some later time and giving them the sheets will allay some of their anxiousness to over-analyze every data point during the interview.

When handling the Overall Culture Assessment page, it is important to note that this is a critical time for exposing interviewees to the Competing Values Framework, as discussed in Chapter 4. Be sure to draw attention effectively to the figure you are describing to prevent overload and increase comprehension. One option built into the design of the worksheet is to fold the Overall Culture Assessment page in half widthwise, and reveal the top half only before progressing to the bottom. This technique was used at Company A to initially supplement inexperience in guiding that conversation. Once interviewees grasp how to read their Overall Culture Assessment page and have read the CVF chart descriptions, discuss all results and allow conversation to flow 
naturally, following the interviewees' lead as they take in the information, probing for any interpretations or impressions that could enrich the data.

Brief Descriptions of Culture Assessment Worksheet Updates. The Itemized Culture Assessment Template was improved after application at Company A. The visual display of OCAI data was difficult to decipher initially, with 24 sets of OCAI profile rendered in data bars of uniform color. A two-color format was added to assist in visually distinguishing between group and individual results, and a vertical midline was added to further accentuate the "now" vs "preferred" data (see figure 26, below). As described in Chapter 6.4, researchers may opt to include a third OCAl results page (by grouping) if the company is interested in having a broader range of data collected. 


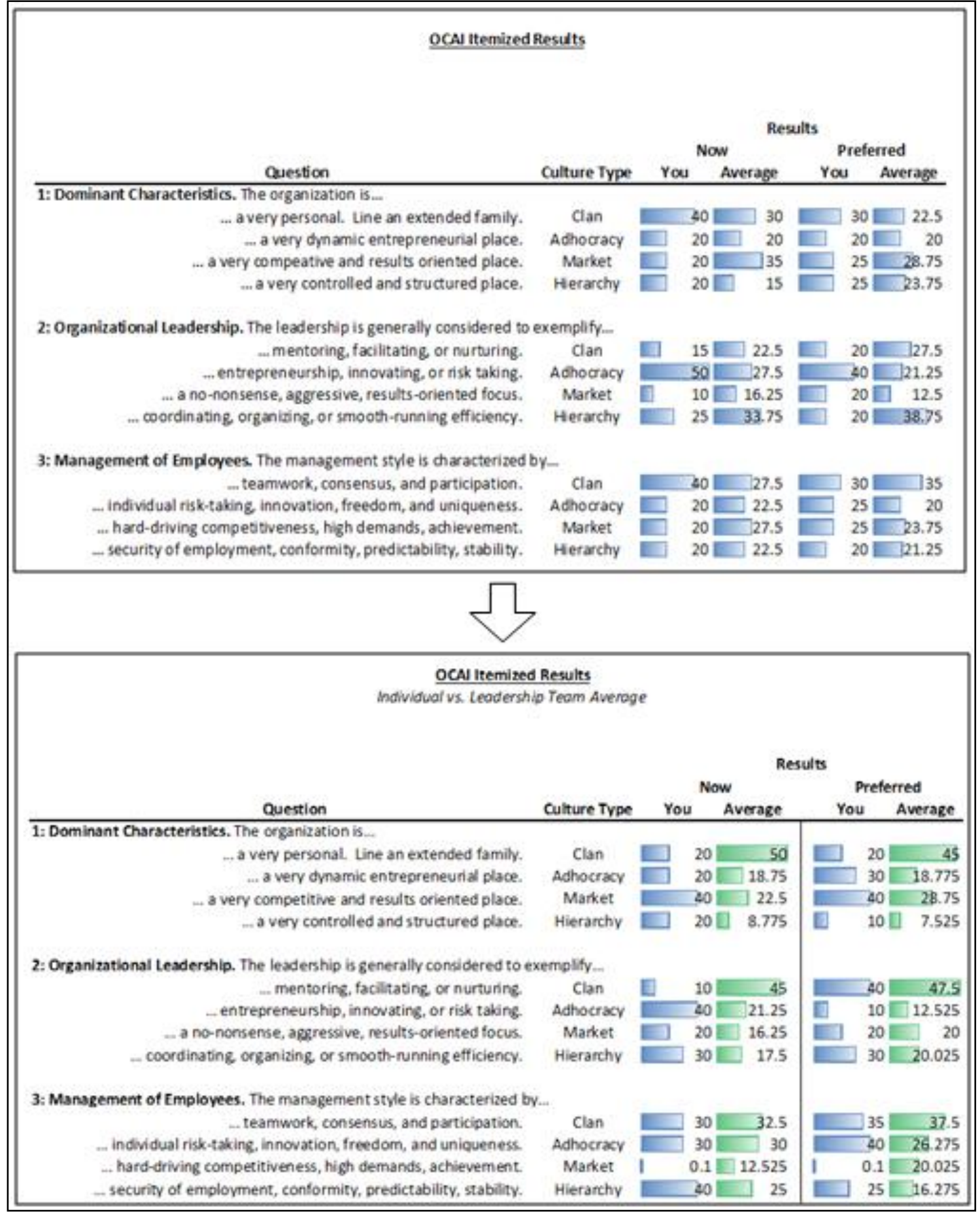

Figure 26: Minor Worksheet Improvements. Figure shows a selection of the initial (top) and final (bottom) Itemized Culture Assessment Template. See Appendix K for final template and full-sized text. 
The "Types of Organizational Values Chart" constructed for this research initially on the Core Values Identification Form was relocated to its own worksheet (See Appendix L) and introduced before the core value identification form is used, in order to better sensitize interviewees to the component concepts prior to discriminating between core and other types of values. This improved ordering helps interviewees to screen out obvious "other" types of core values with minimal prompting during the subsequent core value identification phase of the interview. The chart was also used in the final presentation to inform or remind company leaders of the distinguishing features of the various types of organizational values.

\section{A Brief History of Core Value Candidate Identification and Value-Behavior}

Association. The Core Values Identification Form (CVI) (Appendix M) and ValueBehavior Association Form (V-BA) (Appendix N) changed drastically through the iterative applications and refinements, and functioned extremely well by Company C. The initial CVI included extensive amounts of data (literally 2.5 pages of "candidates" at Company A, including nearly 2 pages of low-to mid- quality candidates) and asked interviewees to select, rank and weight top organizational core value candidates from that list. The initial CVI also failed to provide interviewees with a clear vision of what the final, desired output might look like, which proved to be an important shortcoming. The process was significantly improved through feedback and reflection, which was implemented at Company B (see Figure 27, below). In short, a variation of the initial CVI became the fundamental template for later iterations of the V-BA form. The final CVI iteration became an unofficial Core Values draft or concept sketch, which (a) serves to provide 
interviewees with a clear vision of the final desired output, (b) greatly reduces the volume of material and cognitive energy required to identify and rank top organizational values, and (c) is a more digestible form for interviewees to help with fine-tuning and language suggestions. An overview of these technical changes is depicted in Figure 27, below. 


\section{Company A:}

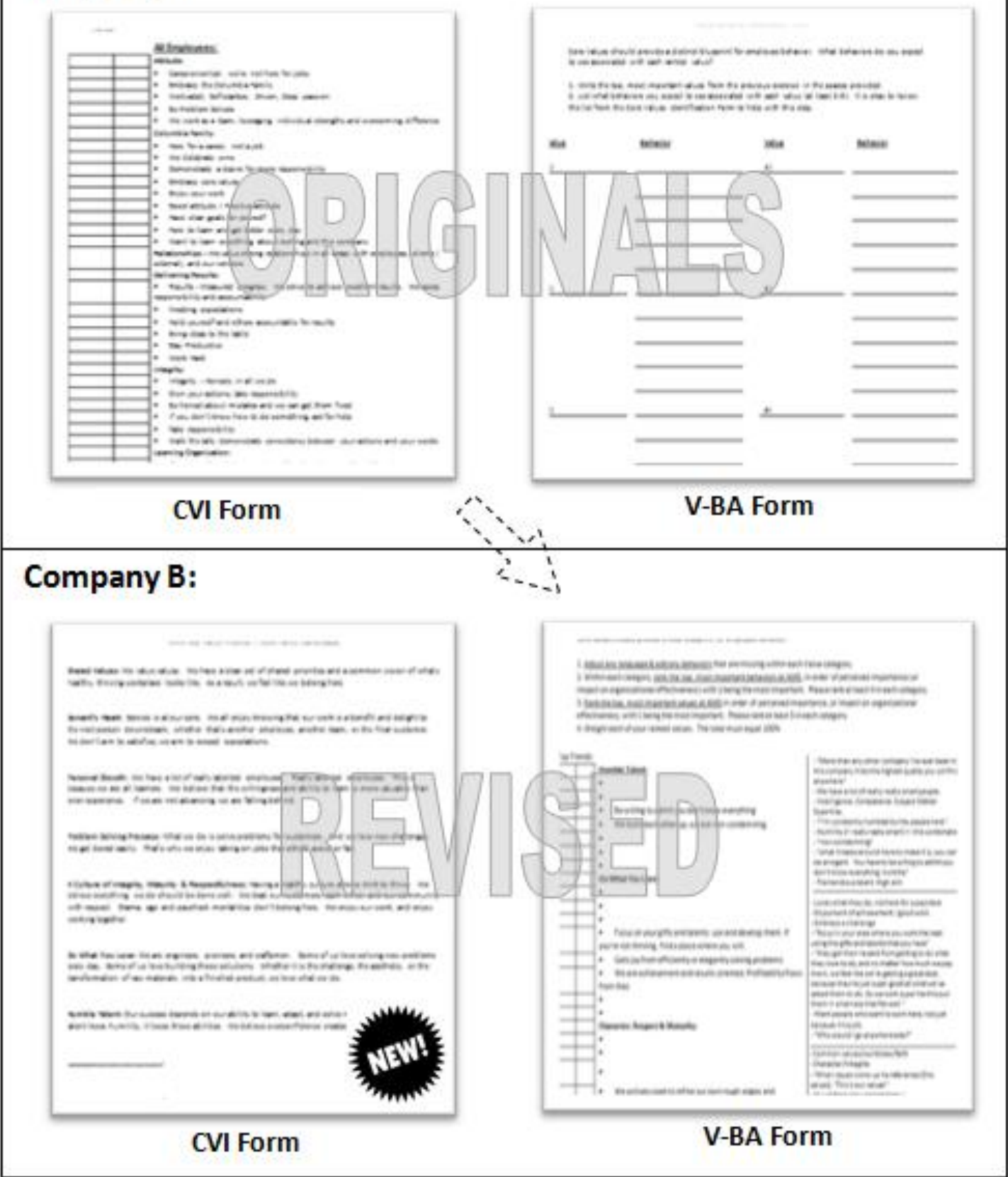

Figure 27: Overview of technical changes to CVI and V-BA forms between Company A and Company B.

The initial CVI form became the essential process template for later iterations of the V-BA form; the initial $V$-BA form became obsolete, and a new CVI template created. The final form templates can be found in Appendices $M$ and $N$.

The history of the process involving the continuous improvement of these forms is more complicated than it appears. The researcher decided to start with the Value- 
Behavior Association exercise before ranking values after reflecting on the Company $\mathrm{A}$ experience, because this reversed process led to a deeper familiarity with the categories and core value candidates before ranking them. This would later change again at Company B, after successfully experimenting with the process of first providing interviewees a trial version of the finished product to improve the incisiveness of the conversation, then having them rank/"order" values, followed finally by associating behaviors. This process worked very well at Company B and C, as evidenced by the relative ease of the process (compared to Company A) and the high quality results.

Weighting is Optional. The usefulness of weighting values and behaviors varied by participant at Company $\mathrm{A}$ and $\mathrm{B}$, and was experimentally omitted at Company $\mathrm{C}$ altogether without any negative impact to the quality of the final core values statement. One example of the useful application of weightings was in an interview with the Company A owner, who confidently declared that "Well without results you don't have anything. That's probably $90 \%$ of it is delivering results." This was a case where the weighting exercise yielded an incisive view into the perspective of this highly influential member of the leadership team. However, some employees embraced the exercise while others did not. Multiple interviewees consistently weighted worksheet items equally to avoid the exercise altogether. Similarly, weighting the wide variety of behaviors was mentally taxing for some interviewees, and their varied levels of investment in the exercise yielded an inconsistent quality of outputs for analysis. As a result, weightings were reconsidered as optional, to be probed at the discretion of the 
researcher. In summary, practitioners should seek to utilize the weighting exercise, but also be willing to replace this exercise in circumstances where an alternative approach might be more productive.

An Option for Reducing Labor-Hours. Note that the vast majority of rich data resulting from this round of interviews appears on the worksheets completed by each interviewee. Recall that the ranking or relationship between values, desired language, associated themes and behaviors should all be marked visibly on the worksheets. For these reasons, practitioners seeking opportunities to reduce the labor-hours involved in the CCVI process should absolutely record the second round of interviews, but may choose to opt out of transcribing these interviews.

\subsection{Step 6: Data Synthesis}

The essential process of Data Synthesis, described in detail in Chapter 4.6, is to synthesize the feedback from the previous round of interviews in order to create a written statement of company Core Values statement. This section contains brief reflections on the process of organizing core value statements and discusses the constructivist approach to grounded theory employed in this work.

Reflections on Organizing Core Values Statements based on feedback.

Synthesizing the second round interview feedback from multiple interviewees was achieved through the application of both quantitative rigor and qualitative synthesis. As described in Chapter 4.6, tables and spreadsheets were constructed to assist the researcher in visually displaying interview feedback. These spreadsheets were iteratively reorganized and improved - systematically, deductively and intuitively - 
based on the full context and content of the applied CCVI Technique up to that point. The immersive nature of qualitative case study methodologies excel at doing this and provide the researcher with a depth of insight that "outsiders" would not likely be able to otherwise achieve.

A key improvement in the CCVI Technique was the eventual decision to allow relational models of Core Values to emerge, in addition to or in place of rankings. The time for realizing this option is in second round interviews, where interviewees should first be asked to rank Core Value Candidates, but should also be probed for relationships between them. This option was innovated after application at Company B, where company leaders had widely divergent perspectives on the prioritization of the top six core value candidates (see Figure 28). Tensions arising from the ranking process led one employee to write at the bottom of the Value-Behavior Association form that "Ranking these is like ranking members of your family - my sense is that they are all inter-related and diminishing the importance of one value takes away from them all (like a body losing members)." However, the rankings received from the leadership team proved critical in distinguishing Core Values from the other highly ranked values. A review of the data and relationships led to the conclusion that the top three ranked values together formed a cohesive foundation which the other important values depend on. The distinction, once made and reviewed, yielded this response in a follow up note from company leadership:

This was a valuable and worthwhile exercise IMHO. You did a good job to survey and summarize what we think are our core values. The categories and the 
characteristics are familiar to read. The process to identify them seemed to work. It's interesting that the range of scores was so wide on \#2 \& 3, which weakens the average. However, I think the top three are prioritized properly and it makes sense that the second three would depend on the first three.

The "top three" referred to in this note are the identified core values (see the top three Core Value Candidates in figure 6.6a, below). The "second three" values are referred to in this note as dependant on the identified core values (see the bottom three values in figure 28 , below). These statements support the theory that the core values were correctly identified and distinguished from the other important values at Company B.

\begin{tabular}{|r|r|r|r|r|r|l|}
\hline \multicolumn{6}{|c|}{ Individual Leader Rankings } & \multicolumn{2}{l|}{$\begin{array}{l}\text { Average } \\
\text { Rankings }\end{array}$} & Core Value Candidates: \\
\hline 3 & \multicolumn{1}{c|}{ B } & \multicolumn{1}{c|}{ C } & \multicolumn{1}{c|}{ D } & \multicolumn{1}{c|}{ E } & Ran \\
\hline 1 & 6 & 2 & 1 & 2 & Do what you love \\
\hline 5 & 4 & 2 & 1 & 3 & 2.6 & Problem Solving DNA \\
\hline 4 & 2 & 4 & 5 & 2 & 3.2 & Culture of Integrity, Maturity \& Respect \\
\hline 2 & 3 & 5 & 3 & 6 & 3.8 & Serving Through Work Well Done \\
\hline 6 & 5 & 6 & 6 & 5 & 3.8 & Humble Confidence \\
\hline
\end{tabular}

Figure 28: Company B Core Values Identification Form Feedback Synthesis. Table reflects real value ranking data collected at Company $B$ through the Core Values Identification forms completed by five company leaders. In this example the top three ranked values were selected as core during Data Synthesis, and later validated by company leadership. Values in gray were considered important but distinct from "core."

Reflection on the tension between ranking and possible relational models prepared the researcher for events at Company $C$, where interviewees again struggled with ranking values during second round interviews. The researcher probed for and discussed the relationships between the values with interviewees, which led to the emergence of a cohesive narrative. From those conversations where company leaders 
struggled to rank values, a new process-oriented relational model of Core Values emerged that gained unanimous support from the company leadership team. That narrative is described in Chapter 5.3 and illustrated through the pictures and descriptions to the left in the Company C Core Values Statement (see Figure 18). While the ranking of core values exercise was replaced at Company $\mathrm{C}$ by these discussions, top associated behaviors were selected and ranked by company leaders, and those rankings synthesized by the researcher during the construction of the Company C Core Values statement.

A key to successfully synthesizing feedback in order to produce a wellconstructed Core Values statement is taking a systematic and deductive approach informed by experience and intuition where necessary to advance the process (Charmaz, 2006). This approach created a sense that the relationships and structuring of the data were emerging naturally rather than being constructed. One unexpected occurrence was that precisely three core values were identified at each company. This fact was coincidental, based solely on the data at each of the three companies independently.

\subsection{Step 7: Final Interviews}

The goals of Final Interviews, as described in Chapter 4.7, are to probe for any feedback regarding the completeness and correctness of the Core Values Statement developed by the researcher in the previous step and discuss any questions or suggestions the practitioner intends to include in the final presentations. The researcher was dissatisfied with the feedback from Company A because every 
participant offered only affirming and positive feedback in regard to the Core Values Statement, but none offered the constructive feedback that was hoped for. In subsequent companies, more pointed effort was made to obtain concrete feedback by informing participants that the researcher is looking for "concrete feedback for improving the document." Despite this emphasis on constructive feedback, Company B and $\mathrm{C}$ leadership did not make any material suggestions for the revision of the provided Core Values Statements during final interviews. However, discussion and feedback regarding other results included in the final presentations were productive and led to improvements in the final presentation for each company.

As described in section 4.7, final interviews were used as opportunities to test, probe or discuss possible suggestions or guidelines for organizational change that were developed in tandem with the assessment of core values. An example of productive discussion impacting the final presentation at Company $A$ was the exclusion of a poster developed by the practitioner, which included important values identified within two subgroups of Company A: office staff and field employees. While different sets of important values were identified for each group, there was apprehension regarding the risk of "increasing the perceived division" between those two groups by presenting those differences.

\subsection{Step 8: Final Presentations}

Final presentations mark the end of the CCVI Technique, where the assessed organizational core values are presented along with insights or suggestions for guiding organizational change. A robust familiarity with each target company - including top 
concerns, aspirations, persistent issues and formal OCAI assessment of their future desired state - was developed in this research through the immersive CCVI Technique, which invariably yielded insights of value to companies. In addition to providing the researcher with these insights, two Company $\mathrm{C}$ employees independently observed that the constant contact with participants also allows the process of guiding organizational change to begin before the presentation takes place. One employee stated after the final presentation that he was, "surprised how many of the suggestions [the researcher] made are things we are already moving into the implementation phase." These leaders believed that the questions and conversations had with employees "seeded" certain ideas and changes in key places, so the well-tempered suggestions delivered during final presentations also found immediate traction (because of those previous conversations). Another example of suggestions well received was evident in a follow-up email from a Company B leader, who wrote:

Your observation about team roles and proper balance was surprisingly insightful. Your analysis, viewed through the lens of Belbin's research, may well help us understand our leadership team dynamics better (which is hugely valuable). We are processing how to make use of this work to make [Co. B] an even better place.

The reference to Belbin's research and team roles in this note was in regard to material included in the final presentation, where the researcher instructively rendered specific suggestions for improving team and work-process functionality by integrating specific knowledge of company processes with R. Meredith Belbin's work on Team Roles at 
Work (Belbin, 2012). Suggestions made by various practitioners after application of the CCVI Technique will vary according to their knowledge, skills and unique histories.

\subsection{Contributions of the Research}

This research is significant because it developed a new technique for broad application (the CCVI Technique), increased academic knowledge, and affected policy at the three subject companies. Each of these contributions will be discussed in turn.

First, the main purpose of this research was to develop a technique for assessing unique organizational core values, based on the Competing Values Framework. There is widespread agreement that identifying core values is essential for guiding culture change and development initiatives (Cameron \& Quinn, 2011; Kotter, 1992; Lencioni, 2002; Porras \& Collins, 1994), and leading scholars and practitioners also emphasize the uniqueness of organizational culture and core values (Cameron \& Quinn, 2011; Collins \& Porras, 1994; Edgeman \& Scherer, 1999; Lee, Olson, \& Trimi, 2012; Lencioni, 2002; Prahalad \& Hamel, 1990; Taylor et al., 2007). However, the OCAl does not capture cultural values unique to an organization. This limitation of the OCAI represents a clear gap between current theory (on the uniqueness of organizational cultures and core values) and practice. The CCVI Technique resulting from this research contributes a specific, practical tool that closes this gap.

Second, this research increases knowledge by empirically supporting the Competing Values Framework (CVF) and producing an emergent, empirically-based extension of the theory. This is significant because the trustworthiness of scientific 
theory depends on replication and empirical support in multiple studies. While the OCAI has been employed thousands of times to assess cultures based on the CVF, the standardized language and categories of the OCAI make the instrument prone to inaccuracies within quadrants or cultural value sets. This research was structured to produce emergent, empirically-based assessments of cultural values based on the Core Values Framework without being limited by the standardized language or accuracy of the OCAI.

Third, this research has significantly affected policy by providing decision makers at three subject companies with in-depth and fresh perspective on their respective core values, current culture, preferred culture, and other employees' perspectives at their firm. Regardless of organizational goals, assessment is essential in strategic planning. Inadequate situational assessment is a frequently cited reason for failure in decision making (Orasanu \& Salas, 1993).

\subsection{Limitations and Future Research}

Many of the limitations of the CCVI Technique presented in this research also provide opportunities for future research. The CCVI Technique has a brief history of application and testing, which is currently limited to: (a) testing at three organizations; (b) small privately owned companies; and, (c) application one practitioner. Finally, the results from each case study are not generalizable, as is often the case, and are reasonably bounded by the researcher's ability to ensure complete contextual accuracy. Each of these limitations and associated opportunities for research are discussed below. 
First, the scope of this research was limited to the application of the CCVI

Technique at three organizations. The possible ineffectiveness of the CCVI Technique in surfacing unique core values was initially acknowledged as a potential limitation, which was addressed by iteratively refining the technique at three companies, and by validating and verifying the results at each company. Future application of the CCVI Technique at other organizations would further verify the usefulness and transferability of the technique in assessing unique core values through the competing values framework, and using that assessment to guide organizational change.

Second, the scope of this research was also limited to the application of the CCVI Technique at small, privately owned companies in Portland, Oregon with fewer than 55 employees. The three participating companies had a total of 110 employees, of which $86 \%$ were Caucasian and $93 \%$ were male. Of the thirteen total participating leaders interviewed at the three companies, $100 \%$ identified themselves as Caucasian and only two were female. These demographic factors present opportunities for future research to explore the application of the CCVI Technique at more ethnically diverse and gender balanced organizations to assess the reliability of the technique under different circumstances. Employee age and education levels at the companies included in this research were more diverse. The researcher did not observe any impacts of age or education level on the employees' ability to participate in the applied research process at the companies included in this research.

Third, the application of the CCVI Technique in this research was also limited to one researcher. There remains a reasonable question of how well other practitioners 
could adopt and apply the technique based on the present research, as the researcher who applied the technique was also the inventor. Future application of the CCVI Technique by other practitioners would therefore further verify the usefulness and transferability of the technique. While the CCVI Technique is not industry specific and is intended for broad use, it is also a time and data intensive technique. This may therefore limit utilization of the technique to practitioners willing to invest the necessary time and resources in the process. Ideal candidates for future applications of the CCVI Technique would include practitioners with experience or training in conducting semi-structured interviews, participant observation, and qualitative data analysis methods. Having an interest in organizational culture and assessment is also an obvious but essential factor to ideal candidacy.

While the CCVI Technique is intended to be used and applied at other organizations, the case study results, as is often the case, are not. This is because the present research sought a qualitative understanding of organizational core values, which are unique to each particular subject company. While qualitative studies excel in such investigations, the results are not generalizable because they are tightly bound to their specific context (Marshall \& Rossman, 2010). To the degree that a company or context outside of this research can be matched to one within this research, some insights may be transferable in those situations.

The accuracy of data capture and analysis in the present applied research was also reasonably constrained by the researcher's ability to accurately capture every possible relevant detail during the project. This limitation includes the risk of possible 
oversights as well as the potential risk of a positivity bias in responses from interviewees concerned with how their responses might reflect the company. Continuous inquiry about the completeness of data collection was used to minimize possible oversights (i.e., asking interviewees if anything important has been overlooked). To minimize the potential impact of positivity bias, case study results were triangulated based on the perspectives of multiple participants from each subject company and member-checking was employed at every interview to ensure accuracy. Participant observation inherently includes the risk of participants changing their behavior based on the presence of a field researcher, sometimes referred to as the Hawthorne effect (McCambridge, Witton, \& Elbourne, 2014). The Hawthorne effect was also mitigated in this research by the use of member-checking and triangulation, two classically employed tactics for employing rigor in qualitative research (Douglas, Johnson, \& Altheide, 1977; Lincoln \& Guba, 1985).

As with all studies, these efforts to minimize bias were not perfect and were marked by tradeoffs. The primary example is that validation of case study results occurred through member-checking with the leadership team only, and other employees were not included. While the perspectives of other employees may have productively contributed to the substance or verification of results, member-checking was constrained to include only the leadership team in order to minimize the burden placed on the workplace such as workflow interruptions, and to allow the leadership team full control over the discussion or dissemination of results to other organizational constituents.

\subsection{Closing Remarks}


As succinctly stated by Hall (2005), "Systems emerge from the values and assumptions of their designers" (p. 115). Core values set our mental benchmarks and provide the basis on which we weigh our options. They lie at the heart of organizational decision making, invisibly tipping the scales of individual choices in their favor. To ignore these most fundamental organizational paradigms when assessing or attempting to change organizational culture is to increase the risk of failure.

As one who straddles the boundary between what we call the academic and professional worlds, I have taken great care to develop a technique that draws upon the best of both. The academic community will find the CCVI Technique both old and new. The technique applies classic methods and well established ideas - such as semistructured interviews, document review, participant observation, Grounded Theory data analysis techniques, and the Competing Values Framework - and synthesizes them in a fresh and nuanced process designed to penetrate to the very core of organizational culture in assessing the unique core - the DNA - of an organization.

Practitioners should find the CCVI Technique practical and effective for assessing unique organizational core values and guiding organizational change. In order to make it accessible for practitioners, the CCVI Technique was deliberately presented stepwise and in straightforward language in Chapter 4. That presentation of the CCVI Technique is also supported by displays and explanations of completed case study results in Chapter 5 , and further supplemented by stepwise discussions that unpack the evolution and rationale of the technique in Chapter 6. Finally, much of the work that was done to make the CCVI Technique as streamlined and effective as possible is now embodied in 
the protocols, templates and worksheets found in the appendices. These documents serve as invaluable resources for both applying and understanding the intricacies of the technique.

In summary, this research concisely presents a technique for assessing organizational core values through a rich, qualitative research design -- and presents the culminating CCVI Technique in a way that practitioners find accessible and academia can embrace. 


\section{List of Works Cited}

Abernethy, M. (2015). Self-reports and Observer Reports as Data Generation Methods: An Assessment of Issues of Both Methods. Universal Journal of Psychology, 3(1), $22-27$.

Albert, S., \& Whetten, D. A. (1985). Organizational identity. Research in Organizational Behavior.

Arnold, D. R., \& Capella, L. M. (1985). Corporate culture and the marketing concept: a diagnostic instrument for utilities. Public Util. Fortn.;(United States), 116(8).

Barney, J. B. (1986). Organizational culture: can it be a source of sustained competitive advantage? Academy of Management Review, 11(3), 656-665.

Barrett, R. (2013). The Values-Driven Organization: Unleashing Human Potential for Performance and Profit. Abingdon, Oxon: Routledge.

Baxter, P., \& Jack, S. (2008). Qualitative case study methodology: Study design and implementation for novice researchers. The Qualitative Report, 13(4), 544-559.

Belbin, R. M. (2012). Team roles at work. Routledge.

Bellot, J. (2011). Defining and assessing organizational culture. In Nursing Forum (Vol. 46, pp. 29-37). Wiley Online Library.

Bernstein, D., \& Audley, R. (1986). Company image and reality: A critique of corporate communications. Cassell.

Blumer, H. (1986). Symbolic interactionism: Perspective and method. Univ of California Press. 
Bourne, H., \& Jenkins, M. (2013). Organizational Values: A Dynamic Perspective. Organization Studies, 34(4), 495-514.

Bowditch, J. L., Buono, A. F., \& Stewart, M. M. (2007). A Primer on Organizational Behavior, 7th Edition (7th edition). Hoboken, NJ: John Wiley \& Sons.

Cameron, K. S., \& Quinn, R. E. (2011). Diagnosing and changing organizational culture: Based on the competing values framework. John Wiley \& Sons.

Carter, N., Bryant-Lukosius, D., DiCenso, A., Blythe, J., \& Neville, A. J. (2014). The Use of Triangulation in Qualitative Research. In Oncology nursing forum (Vol. 41, pp. 545-547). Onc Nurs Society.

Charmaz, K. (2006). Constructing grounded theory: A practical guide through qualitative research. London: Sage.

Collins, J. C., \& Porras, J. I. (1994). Built to last. HarperBusiness.

Corbin, J., \& Strauss, A. (2007). Basics of Qualitative Research: Techniques and Procedures for Developing Grounded Theory. SAGE.

Corbin, J., \& Strauss, A. (2014). Basics of qualitative research: Techniques and procedures for developing grounded theory. Sage publications.

Denison, D. R. (1996). What Is the Difference Between Organizational Culture and Organizational Climate? A Native's Point of View on a Decade of Paradigm Wars. Academy of Management Review, 21(3), 619-654.

De Vries, M. F. K., \& Miller, D. (1986). Personality, culture, and organization. Academy of Management Review, 11(2), 266-279. 
DeWalt, K. M., \& DeWalt, B. R. (2010). Participant observation: A guide for fieldworkers. Rowman Altamira.

Douglas, J. D., Johnson, J. M., \& Altheide, D. L. (1977). Existential sociology. Cambridge University Press New York.

Edgeman, R. L., \& Scherer, F. (1999). Systemic leadership via core value deployment. Leadership \& Organization Development Journal, 20(2), 94-98.

Ernest, R. C. (1985). Corporate cultures and effective planning. Personnel Administrator. Gair, S. (2012). Feeling Their Stories Contemplating Empathy, Insider/Outsider Positionings, and Enriching Qualitative Research. Qualitative Health Research, 22(1), 134-143.

Glaser, B. (1978). Theoretical sensitivity: Advances in the methodology of grounded theory. Sociology Pr.

Glaser, B. (1998). Doing grounded theory: Issues and discussions. Sociology Press.

Glaser, B., \& Strauss, A. (1967). The discovery grounded theory: strategies for qualitative inquiry. London, England: Wiedenfeld and Nicholson.

Guba, E., \& Lincoln, Y. (1985). Effective evaluation: improving the usefulness of evaluation results through responses and naturalist approaches. San Francisco: Jossey-Bass.

Hall, D. E. (2005). Development and validation of the Sustainability Climate Survey (Unpublished master's thesis). Portland State University. Harrison, M. I. (2005). Diagnosing organizations: Methods, models, and processes (Vol. 8). Sage. 
Harrison, \& Shirom, A. (1999). Organizational diagnosis and assessment: Bridging theory and practice. Sage.

Hofstede, G. (1980). Culture's consequences. Beverly Hills. Ca: Sage.

Hogan, S. J., \& Coote, L. V. (2014). Organizational culture, innovation, and performance: A test of Schein's model. Journal of Business Research, 67(8), 1609-1621.

Hume, L., \& Mulcock, J. (2013). Anthropologists in the field: Cases in participant observation. Columbia University Press.

Jahoda, G. (2012). Critical reflections on some recent definitions of "culture." Culture \& Psychology, 18(3), 289-303.

Kilmann, R. H., Saxton, M. J., \& Serpa, R. (1985). Gaining control of the corporate culture. Jossey-Bass Inc Pub.

Kotter, J. P. (1992). Heskett. JL (1992). Corporate Culture and Performance. New York: Free Press.

Lee, S. M., Olson, D. L., \& Trimi, S. (2012). Co-innovation: convergenomics, collaboration, and co-creation for organizational values. Management Decision, 50(5), 817831.

Lencioni, P. (2002). Make your values mean something. Harvard Business Review, 80(7), 113-117.

Lencioni, P. (2006). The five dysfunctions of a team. John Wiley \& Sons.

Lencioni, P. (2012). The Advantage: Why Organizational Health Trumps Everything Else In Business. John Wiley \& Sons.

Lincoln, Y. S., \& Guba, E. G. (1985). Naturalist inquiry. Beverly Hills, CA: Sage. 
Marcus, S. E. (2004). Strategic Decision Making in Organizations that Value Financial, Social, and Environmental Sustainability (Doctoral dissertation). Portland State University.

Marshall, C., \& Rossman, G. B. (2010). Designing qualitative research. Sage.

McCambridge, J., Witton, J., \& Elbourne, D. R. (2014). Systematic review of the Hawthorne effect: new concepts are needed to study research participation effects. Journal of Clinical Epidemiology, 67(3), 267-277.

Meglino, B. M., \& Ravlin, E. C. (1998). Individual Values in Organizations: Concepts, Controversies, and Research. Journal of Management, 24(3), 351-389.

Morse, J. M., Barrett, M., Mayan, M., Olson, K., \& Spiers, J. (2008). Verification strategies for establishing reliability and validity in qualitative research. International Journal of Qualitative Methods, 1(2), 13-22.

Orasanu, J., \& Salas, E. (1993). Team decision making in complex environments.

Pant, P. N., \& Lachman, R. (1998). Value incongruity and strategic choice. Journal of Management Studies, 35(2), 195-212.

Prahalad, C. K., \& Hamel, G. (1990). The core competence of the corporation. Harvard Business Review, 68(3), 79-91.

Quinn, R. E. (1988). Paradox and transformation: Toward a theory of change in organization and management. Ballinger Publishing Company.

Quinn, R. E., \& Rohrbaugh, J. (1983, March 1). A Spatial Model of Effectiveness Criteria: Towards a Competing Values Approach to Organizational Analysis [researcharticle]. 
Rico, G. L. (1983). Writing the natural way: Using right-brain techniques to release your expressive powers. Tarcher.

Sathe, V. (1985). Culture and related corporate realities. Homewood, IL: Irwin.

Schein, E. H. (1992). Coming to a new awareness of organizational culture. Sage.

Schwartz, S. H. (2012). An overview of the Schwartz theory of basic values. Online Readings in Psychology and Culture, 2(1), 11.

Spradley, J. P., \& Baker, K. (1980). Participant observation (Vol. 195). Holt, Rinehart and Winston New York.

Taylor, G. D., Van Aken, E. M., \& Smith-Jackson, T. (2007). IMPROVING ORGANIZATIONAL CULTURE USING CORE VALUES.

Triandis, H. C. (2004). The Many Dimensions of Culture. The Academy of Management Executive (1993-2005), 18(1), 88-93.

Van Rekom, J., Van Riel, C. B. M., \& Wierenga, B. (2006). A Methodology for Assessing Organizational Core Values. Journal of Management Studies, 43(2), 175-201. 


\section{Appendix A: Study Participant Recruitment Letter}

Dear [prospective subject's name]:

Our names are Wayne Wakeland, PhD and Anthony Santoriello, MBA, and we are a faculty member and graduate student, respectively, at Portland State University. We are beginning a study to explore organizational values in local companies, and would like to invite you and [your company name] to participate.

You are being asked to take part because you and [your company name] have been recommended to us by local business leaders, who identified you as intentional about your company culture and values. As part of the study, we are interested in interviewing your leadership team and observing normal operations for about one week, and hope that the information we collect will help us to better understand the culture and core values of your organization acknowledged by your peers.

If you decide to participate, you will be asked to:

- participate in a series of three interviews arranged at your convenience at the location of your choice in the greater Oregon area

- allow your leadership team to participate in the same series of three interviews arranged at their convenience at the location of their choice in the greater Oregon area

- allow Anthony Santoriello, MBA, to spend approximately 1 week as a student observer at [your company name].

The interviews will involve answering questions about culture description, cultural fit, personal and professional values, and what is valued within the company. It should take approximately one hour to complete one interview session, and we will accommodate our schedule to your convenience to make it as easy as possible for you to sit with us for approximately three interviews.

During this study, you may be uncomfortable being audiotaped or may not have enough time for the interviews. According to your preference, we can delete any portion of the recording you designate at any time in the course of the study (during or after). 
However, we assure you that any information that is obtained in connection with this study and that can be linked to you or identify you will be kept confidential at all times unless you explicitly agree to otherwise (for example, if you decide to be included in a published case study or article about your company and give explicit written consent to do so). You may not receive any direct benefit from taking part in this study, but the study may help participating [your company name] leaders gain a better understanding of the culture and core values at [your company name], and may help academics gain a better understanding of the culture and core values operating in local companies.

Any information that is obtained in connection with this study and that can be linked to or identify yourself or your company will be kept confidential. This information will be kept confidential by storing the tapes at PSU in a locked facility and using transcriptions only (no audio) for research publication or conference presentations unless you have given us explicit and written permission to do otherwise.

Participation is entirely voluntary. Your decision to participate or not will not affect your relationship with the researcher or with Portland State University in any way. If you decide to take part in the study, you may choose to withdraw your participation or the participation of [your company name] at any time without penalty. Please keep a copy of this letter for your records.

If you have concerns or problems about your participation in this study or your rights as a research subject, please contact the Human Subjects Research Review Committee, Office of Research and Sponsored Projects, 1600 SW 4th Avenue, Suite 210, Portland State University, (503) 725-9900. If you have questions about the study itself, contact Anthony Santoriello at anthony.santoriello@pdx.edu or (503) 933-0498.

Sincerely,

Wayne Wakeland, PhD and Anthony Santoriello, MBA

Portland State University 


\title{
Appendix B: Owner Informed Consent Request
}

\author{
Informed Consent for Dissertation Research Project Participation: \\ Exploring Core Values in Local Companies
}

You are invited to participate in a research study conducted by Anthony Santoriello, MBA from Portland State University, Systems Science Program. The researchers hope to learn more about the organizational values in local companies who are recognized as intentional about their culture and values. We would like to include [company name] in this study, and you were identified as the owner or decision maker for [company name] in regards to this study.

If you decide to allow [company name] to participate, you will be asked to

- participate in a series of three interviews arranged at your convenience at the location of your choice in the greater Oregon area

- allow your leadership team to participate in the same series of three interviews arranged at their convenience at the location of their choice in the greater Oregon area

- allow Anthony Santoriello, MBA, to spend approximately 1 week as a student observer at [your company name].

Interviews will be arranged at your (or participating leaders) convenience, at the location of your/their choice in the greater Oregon area. Audio recordings are taken for the sole purpose of transcribing the interviews. Any information that could be linked to you, a leader, or the company will be kept confidential at all times unless you give explicit and written permission to do otherwise. All discussion of results will always protect the identities of the participants, and only transcriptions will be used (no audio) for research publication or conference presentations unless you have given us explicit and written permission to do otherwise.

The week of student observation would include Anthony Santoriello being on-site at [company name] to observe normal day-to-day operations without interrupting workflow or requiring much supervision or assistance. Employees may be asked simple, informal questions you would expect from a student observer - about their day-to-day work and the company in general.

All information will be kept confidential by storing all recordings, notes, transcriptions and data in a locked facility at the home office of the student investigator and/or a locked 
cabinet in the PSU Systems Science Program building for the duration of the study and for three years thereafter. Digital data will be stored on a password protected personal drive and/or the PSU network drive.

According to your preference, we can delete any portion of the data you designate at any time in the course of the study (during or after). We will accommodate our schedule to your convenience to make it as easy as possible for you to sit with us for approximately three interviews. You may not receive any direct benefit from taking part in this study, but the study may help to increase knowledge that may help others in the future. If you would like a copy of your interviews, we can create an mp3 for you.

Your decision to allow [company name] participate or not is entirely voluntary and will not affect your relationship with the researcher or with Portland State University in any way. If you decide to allow [company name] to take part in the study, you may choose to withdraw participation at any time without penalty. Please keep a copy of this letter for your records.

If you have concerns or problems about [company name]'s participation in this study or your rights as a research subject, please contact the Human Subjects Research Review Committee, Office of Research and Sponsored Projects, 1600 SW 4th Avenue, Suite 210, Portland State University, (503) 725-9900. If you have questions about the study itself, contact Anthony Santoriello at anthony.santoriello@pdx.edu or (503) 933-0498.

Your signature indicates that you have read and understand the above information and agree to allow [company name] to take part in this study. Please understand that you may withdraw your consent at any time without penalty, and that, by signing, you are not waiving any legal claims, rights or remedies. The researcher will provide you with a copy of this form for your own records.

Company Name

Your Printed Name

Signature

Date 


\title{
Appendix C: Interviewee Informed Consent Request
}

\author{
Informed Consent for Dissertation Research Project Participation: \\ Exploring Core Values in Local Companies
}

Dear [Participants Name],

I am a graduate student in the Systems Science Program at Portland State University. I would like to invite you to participate in a research project focused on exploring organizational values in local companies that are intentional about their culture and values. [company name] has been included in this study, and you were selected as a possible interview participant in this study because you are a leader at [company name].

If you decide to participate, your participation will include a set of approximately three one-hour interviews arranged at your convenience at the location of your choice in the greater Oregon area. Audio recordings will be taken for the purpose of transcribing the interviews. Your participation is voluntary and you can request to have any or all information you share destroyed at any time. Any information that could be linked to you will be kept confidential at all times unless you give explicit and written permission to do otherwise.

All discussion of results will always protect the identities of the participants. Protection measures will include the use of pseudonyms in any transcriptions used for research publication or conference presentations unless you have given us explicit and written permission to do otherwise. Information will also be kept confidential by storing all recordings, notes, transcriptions and data in a locked container at my home office and/or a locked cabinet in the PSU Systems Science Program building for the duration of the study and for three years thereafter. Digital data will be stored on a password protected personal drive and/or the PSU network drive.

You may not receive any direct benefit from taking part in this study, but the study may help to increase knowledge that could help others in the future. Your participation is entirely voluntary. Your decision to participate or not will not affect your relationship with the researcher or with Portland State University in any way. If you decide to take part in the study, you may choose to withdraw your participation at any time without penalty.

If you have concerns or problems about your participation in this study or your rights as a research subject, please contact the Human Subjects Research Review Committee, Office of Research and Sponsored Projects, 1600 SW 4th Avenue, Suite 210, Portland State University, (503) 725-9900. If you have questions, please feel free to contact me at (503) 933-0498 or anthony.santoriello@pdx.edu. You may also contact my committee chairperson, Professor Wayne Wakeland at wakeland@pdx.edu. The researcher will provide you with a copy of this form for your own records.

Thank you, Anthony Santoriello, MBA

PhD Candidate, Portland State University 
(signed)

Please sign below if you have read and understand the above information and are willing to participate in the dissertation project outlined above. Please understand that by signing you are not waiving any legal claims, rights or remedies.

Printed Name

Signature

Date 


\section{Appendix D: Initial Interview Protocol}

Materials to bring:

$\begin{array}{ll}2 \times \text { Informed Consent } & \text { Demographic Questionnaire } \\ \text { Initial Interview Protocol } & \text { Recorder } \\ \text { OCAI } & \text { Pens } \\ \text { Initial Interview Worksheet } & \text { Notebook }\end{array}$

Oral Disclosure:

(Give participant the two Informed Consent Letters. One is for their records.)

(-) ${ }^{19}$ I'd like to interview you today about your company's culture. I have spent considerable time studying the importance of culture and values - or the "human" component - of businesses, and I have been told that [name of company] is intentional about them.

My ultimate objective here is to understand how you, a leader at [name of company], understand its' culture and values. Alright?

As stated in the informed consent letter I shared with you, any information from this interview that could be linked to you will be kept confidential unless you give explicit and written consent to do otherwise. Your participation is voluntary and you can request to have any or all information you share destroyed at any time. Any such decisions will not affect your relationship with the researchers or with Portland State University.

(Administer Demographic Questionnaire)

With your permission I would like to record our interview so I can transcribe it later, and this is the only purpose for the recording. It will not be shared with anyone.

[Recorder On] 
This isn't going to take more than an hour, and if you want to decline any questions or anything like that it's totally OK. Again, my ultimate objective here is to understand how you, a leader at [name of company], understand its' culture and values. (-) And my goal for you is that you come away refreshed and energized by this conversation.

Interview Start Up Questions

2. $:{ }^{19}$ Tell me, what is one piece of advice you would give to a new employee or applicant about how to thrive at this company?

a. Probe: Is there any other advice you would give to that new employee or applicant about how to thrive here?

3. Can we do a Core Values Assessment Exercise? (Great. I'm going to ask you about 3 sets of employees: provide them with "Interview CVA Worksheet.")

a. Tell me about the best employees or coworkers you've ever had here? No names, but what are some of the VALUES OR BEHAVIORS of people, past or present, who fit really well with the culture - the people who represent the best of the organization?

b. How about some of the VALUES OR BEHAVIORS of "not-so-hot" employees the people who haven't fit well with the culture. No names, but what are some of qualities of the people who haven't fit well with the culture?

C. Can you tell me about the leadership team? No names, but what are the VALUES OR BEHAVIORS the leadership team that really shape the culture?

d. Probe: Tell me, do you see any relationships between any of these values or behaviors we've talked about?

4. Is there anything else you'd like to tell me?

5. One more question for you, and it's my favorite: What's the best part about working here that I won't be able to see from just a walk around the place?

\footnotetext{
${ }^{19}$ A reminder to the researcher to smile and be aware of body language and tone of voice during the interview.
} 
6. Finally, would you mind taking a few more minutes to complete an assessment called the Organizational Culture Assessment Instrument (OCAI)?

The OCAl, shown in Appendix E, will be handed to interviewees in printed form during the interview. The printed OCAl will include instructions, and the following excerpts from the instructions also read to participants to ensure comprehension:

The purpose of the OCAI is to assess six key dimensions of organizational culture. In completing the instrument, you will be providing a picture of how your organization operates and the values that characterize it. There are only 2 pages, and six questions. Each question has four alternatives. Divide 100 points among these four alternatives depending on the extent to which each alternative is similar to your own organization. Give a higher number of points to the alternative that is most similar to your organization. Just be sure your total equals 100 points for each question.

Note, that you will make 2 passes through these questions. On the first pass through the six questions complete the column labeled "Now." This refers to the culture, as it exists today. After you complete the "Now," you will find the questions repeated under a heading of "Preferred." Your answers to these questions should be based on how you would like the organization to look five years from now.

There are no right or wrong answers for these questions, just be as accurate as you can in responding to the questions so that your resulting cultural diagnosis will be as precise as possible. 


\section{Appendix E: Demographic Questionnaire}

\section{Demographic Survey Questions}

Q. Employment Term: How long have you been employed at your current company?

Q. Sex: What is your gender?

$\square \quad$ Female

$\square \quad$ Male

Q. Age: What is your age?

$\square \quad 18-24$ years old

$\square \quad 25-34$ years old

$\square \quad 35-44$ years old

$\square \quad$ 45-54 years old $\square \quad 55-64$ years old

$\square \quad$ 65-74 years old

$\square \quad 75$ years or older

Q. Ethnicity origin (or Race): Please specify your ethnicity.

$\square \quad$ White or Caucasian

$\square \quad$ Hispanic or Latino

$\square$ Black or African American

$\square$ Native American or American Indian

$\square$ Asian / Pacific Islander

$\square$ Other

Q. Education: What is the highest degree or level of school you have completed? If currently enrolled, highest degree received.

$\square \quad$ No high school diploma or equivalent

$\square$ High school graduate, diploma or the equivalent (for example: GED)

$\square \quad$ Some college credit, no degree

Trade/technical/vocational training

$\square \quad$ Associate degree

$\square$ Bachelor's degree

$\square \quad$ Master's degree

$\square \quad$ Professional degree

$\square \quad$ Doctorate degree

Q. Position: What is your current job title at the company where you are employed? 
$\square$ President

$\square \quad$ Vice President

$\square$ General Manager

$\square$ Director

$\square$ Functional Manager

$\square$ Coordinator/Supervisor/Administrator

$\square$ Other 


\section{Appendix F: Initial Interview Worksheet}

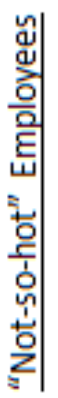




\section{Appendix G: Organizational Culture Assessment Instrument \& Scoring Sheet}

\section{Organizational Culture Assessment Instrument}

Cameron/Quinn, Diagnosing and Changing Organizational Culture, (c) 2000

Instructions for completing the Organizational Culture Assessment Instrument (OCAI).

The purpose of the OCAI is to assess six key dimensions of organizational culture. In completing the instrument, you will be providing a picture of how your organization operates and the values that characterize it. No right or wrong answers exist for these questions, just as there is no right or wrong culture. Every organization will most likely produce a different set of responses. Therefore, be as accurate as you can in responding to the questions so that your resulting cultural diagnosis will be as precise as possible.

You are asked to rate your organization in the questions. To determine which organization to rate, you will want to consider the organization that is managed by your boss, the strategic business unit to which you belong, or the organizational unit in which you are a member that has clearly identifiable boundaries. Because the instrument is most helpful for determining ways to change the culture, you'll want to focus on the cultural unit that is the target for change. Therefore, as you answer the questions, keep in mind the organization that can be affected by the change strategy you develop.

The OCAI consists of six questions. Each question has four alternatives. Divide 100 points among these four alternatives depending on the extent to which each alternative is similar to your own organization. Give a higher number of points to the alternative that is most similar to your organization. For example, in question one, if you think alternative $A$ is very similar to your organization, alternative $B$ and $C$ are somewhat similar, and alternative $D$ is hardly similar at all, you might give 55 points to $A, 20$ points to $B$ and $C$, and five points to $D$. Just be sure your total equals 100 points for each question.

Note, that the first pass through the six questions is labeled "Now." This refers to the culture, as it exists today. After you complete the "Now," you will find the questions repeated under a heading of "Preferred." Your answers to these questions should be based on how you would like the organization to look five years from now. 


\section{The Organizational Culture Assessment Instrument}

\begin{tabular}{|c|c|c|c|}
\hline \multicolumn{2}{|r|}{ 1. Dominant Characteristics } & \multirow{2}{*}{ Now } & \multirow[t]{2}{*}{ Preferred } \\
\hline A & $\begin{array}{l}\text { The organization is a very personal place. It is like an extended family. } \\
\text { People seem to share a lot of themselves. }\end{array}$ & & \\
\hline B & $\begin{array}{l}\text { The organization is a very dynamic entrepreneurial place. People are } \\
\text { willing to stick their necks out and takerisks. }\end{array}$ & & \\
\hline $\mathrm{C}$ & $\begin{array}{l}\text { The organization is very results oriented. A major concern is with } \\
\text { getting the job done. People are very competitive and achievement } \\
\text { oriented. }\end{array}$ & & \\
\hline $\mathrm{D}$ & $\begin{array}{l}\text { The organization is a very controlled and structured place. Formal } \\
\text { procedures generally govern what people do. }\end{array}$ & & \\
\hline & Total & & \\
\hline \multicolumn{2}{|r|}{ 2. Organizational Leadership } & Now & Preferred \\
\hline A & $\begin{array}{l}\text { The leaders hip in the organization is generally considered to exemplify } \\
\text { mentoring, facilitating, or nurturing }\end{array}$ & & \\
\hline B & $\begin{array}{l}\text { The leaders hip in the organization is generally considered to exemplify } \\
\text { entrepreneurship, innovating, or risk taking. }\end{array}$ & & \\
\hline $\mathrm{C}$ & $\begin{array}{l}\text { The leaders hip in the organization is generally considered to exemplify a } \\
\text { no-nonsense, aggressive, results-oriented focus. }\end{array}$ & & \\
\hline $\mathrm{D}$ & $\begin{array}{l}\text { The leaders hip in the organization is generally considered to exemplify } \\
\text { coordinating organizing, or smooth-running efficiency. }\end{array}$ & & \\
\hline & Total & & \\
\hline \multicolumn{2}{|r|}{ 3. Management of Employees } & Now & Preferred \\
\hline A & $\begin{array}{l}\text { The management style in the organizationis characterized by teamwork, } \\
\text { consensus, and participation. }\end{array}$ & & \\
\hline$B$ & $\begin{array}{l}\text { The management style in the organizationis characterized by individual } \\
\text { risk-taking, innovation, freedom, and uniqueness. }\end{array}$ & & \\
\hline $\mathrm{C}$ & $\begin{array}{l}\text { The management style in the organizationis characterized by hard- } \\
\text { driving competitiveness, highdemands, and achievement. }\end{array}$ & & \\
\hline $\mathrm{D}$ & $\begin{array}{l}\text { The management style in the organizationis characterized by security of } \\
\text { employment, conformity, predictability, and stability in relationships. }\end{array}$ & & \\
\hline & Total & & \\
\hline
\end{tabular}




\begin{tabular}{|c|c|c|c|}
\hline \multicolumn{2}{|c|}{ 4. Organization Glue } & \multirow[t]{2}{*}{ Now } & \multirow[t]{2}{*}{ Preferred } \\
\hline A & $\begin{array}{l}\text { The glue that holds the organization together is loyalty and mutual trust. } \\
\text { Commitment to this organization runs high. }\end{array}$ & & \\
\hline $\mathrm{B}$ & $\begin{array}{l}\text { The glue that holds the organization together is commitment to } \\
\text { innovation and development. There is an emphasis on being on the } \\
\text { cutting edge. }\end{array}$ & & \\
\hline C & $\begin{array}{l}\text { The glue that holds the organization together is the emphasis on } \\
\text { achievement and goal accomplishment. Aggressiveness and winning } \\
\text { are common themes. }\end{array}$ & & \\
\hline \multirow[t]{2}{*}{ D } & $\begin{array}{l}\text { The glue that holds the organization together is formal rules and } \\
\text { policies. Maintaining a smooth-running organization is important. }\end{array}$ & & \\
\hline & Total & & \\
\hline \multicolumn{2}{|c|}{ 5. Strategic Emphases } & Now & Preferred \\
\hline A & $\begin{array}{l}\text { The organization emphasizes human development. High trust, } \\
\text { openness, and participation persist. }\end{array}$ & & \\
\hline $\mathrm{B}$ & $\begin{array}{l}\text { The organization emphasizes acquiring new resources and creating new } \\
\text { challenges. Trying new things and prospecting for opportunities are } \\
\text { valued. }\end{array}$ & & \\
\hline $\mathrm{C}$ & $\begin{array}{l}\text { The organization emphasizes competitive actions and achievement. } \\
\text { Hitting stretch targets and winning in the marketplace are dominant. }\end{array}$ & & \\
\hline \multirow[t]{2}{*}{ D } & $\begin{array}{l}\text { The organization emphasizes permanence and stability. Efficiency. } \\
\text { control and smooth operations are important. }\end{array}$ & & \\
\hline & Total & & \\
\hline \multicolumn{2}{|c|}{ 6. Criteria of Success } & Now & Preferred \\
\hline A & $\begin{array}{l}\text { The organization defines success on the basis of the development of } \\
\text { human resources, teamwork, employee commitment, and concern for } \\
\text { people. }\end{array}$ & & \\
\hline B & $\begin{array}{l}\text { The organization defines success on the basis of having the most } \\
\text { unique or newest products. It is a product leader and innovator. }\end{array}$ & & \\
\hline $\mathrm{C}$ & $\begin{array}{l}\text { The organization defines success on the basis of winning in the } \\
\text { marketplace and outpacing the competition. Competitive market } \\
\text { leadership is key. }\end{array}$ & & \\
\hline$D$ & $\begin{array}{l}\text { The organization defines success on the basis of efficiency. } \\
\text { Dependable delivery, smooth scheduling and low-cost production are } \\
\text { critical. }\end{array}$ & & \\
\hline & Total & & \\
\hline
\end{tabular}




\section{A Worksheet for Scoring the OCAI}
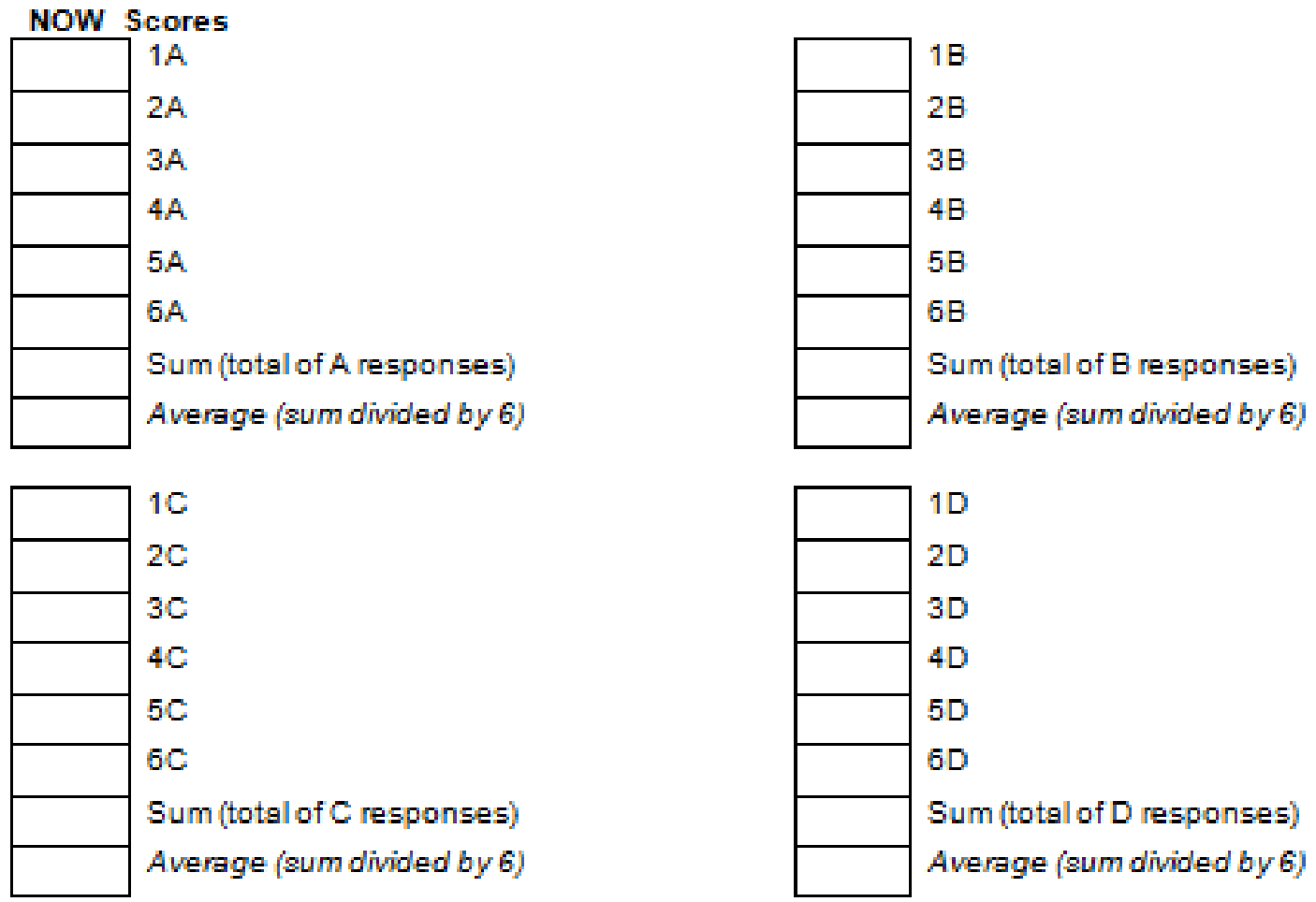

PREFERRED Scores
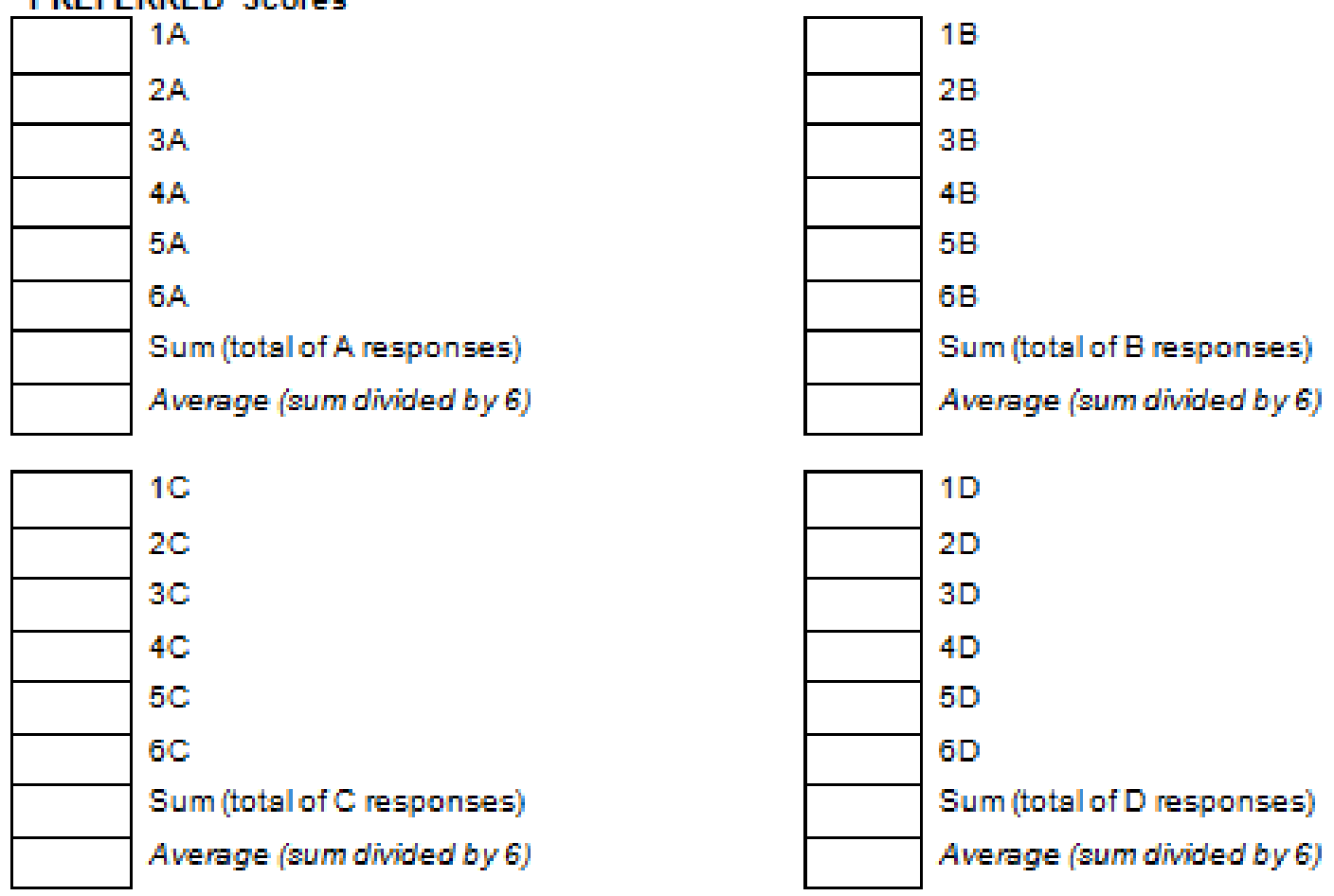


\section{Scoring}

Scoring the OCAI is very easy. It requires simple arithmetic calculations. The first step is to add together all $A$ responses in the Now column and divide by six. That is, compute an average score for the $A$ alternatives in the Now column. You may use the worksheet on the next page to arrive at these averages. Do this for all of the questions, A, B, C, and $D$. Once you have done this, transfer your answers to this page in the boxes provided below.

Fill in your answers here from the previous page

\begin{tabular}{|l|l|}
\hline \multicolumn{2}{|l|}{ NOW } \\
\hline A(Clan) & \\
\hline B (Adhocracy) & \\
\hline C (Market) & \\
\hline D (Hierarchy) & \\
\hline Total & \\
\hline
\end{tabular}

\begin{tabular}{|l|l|}
\hline \multicolumn{2}{|l|}{ PREFERRED } \\
\hline A (Clan) & \\
\hline B (Adhocracy) & \\
\hline C (Market) & \\
\hline D (Hierarchy) & \\
\hline Total & \\
\hline
\end{tabular}

\section{An Example of How Culture Ratings Might Appear}

\begin{tabular}{|l|c|}
\hline \multicolumn{2}{|l|}{ NOW } \\
\hline A & 55 \\
\hline B & 20 \\
\hline C & 20 \\
\hline D & 5 \\
\hline Total & 100 \\
\hline
\end{tabular}

\begin{tabular}{|l|c|}
\hline \multicolumn{2}{|l|}{ PREFERRED } \\
\hline A & 35 \\
\hline B & 30 \\
\hline C & 25 \\
\hline D & 10 \\
\hline Total & 100 \\
\hline
\end{tabular}




\section{Appendix H: Participant Observation Field Guide}

\section{Purpose of Participant Observation:}

Address A-RQ\#1b ("What values are reflected in the behaviors of the employees?"). This data will enriched the collection of organizational values at target companies and will be used later in the process of identifying unique core values.

\section{Participant Observation Checklist:}

1. Physical:

$\square$ Observe status symbols embedded in offices, hallways, meeting rooms, workspaces.

$\square$ Record observations from dress/dress codes, banners, newsletters.

$\square$ Observe and record demographic information.

2. Verbal:

$\square \quad$ Observe and Record behaviors reflected in greetings, interactions, casual conversations, formal meetings or speeches, "the grapevine."

$\square \quad$ If any new employees, observing them carefully can be a great source of anecdotal information as they are in the process of actively adjusting to the culture. (Buch \& Wetzel 2001)

$\square \quad$ Record any data volunteered by participants

3. Field Notes

Write up formal field notes after each participant observation the same day (or within 24 hours).

\section{Essential Observation Process Flow:}

- Establish Scope \& Schedule

-Introduction to participants

-Establish Trust

-Take Notes:

- Take Notes on Physical Culture

- Take notes on Meetings

- Take notes on Interactions

- Take notes on Behaviors

- Take notes on Processes 


\section{Appendix I: Second Interview Protocol}

Materials to bring:

Second Interview Protocol

Overall Culture Assessment Page

Itemized Culture Assessment Page

Group OCAI Itemized Results ${ }^{20}$
Types of Organizational Values Diagram

Core Values Candidate List

Value-Behavior Association \& Ranking Form

Recorder, Pens, Notebook, Timepiece

\section{Step 1: Oral Introduction:}

$0^{21}$ With your permission I would like to record our interview again so I can transcribe it later.

\section{[Recorder On]}

The goals of this meeting are

1) to share with you the results of this study so far and

2) to complete an exercise that will help me gain a clearer picture of the cultural priorities

3) and it's my goal for you is that you come away refreshed and energized by this conversation.

This is going to be interesting, it won't take more than an hour, and if you want to decline any questions or anything like that once again it's totally OK. And we have a lot of material to cover so we are going to move quickly.

\section{Step 2: Share Overall Culture Assessment page.}

- Prompt: There's no need to fully grasp everything about the results; just get the idea.

- Prompt/Probe: Intro OCAI -> Read over those 4 culture types; Any thoughts on those?

- Discuss key results and allow conversation to flow naturally, following the interviewees lead as they take in the information.

\section{Step 3: Share Itemized Culture Assessment Page.}

- Discuss key results and allow conversation to flow naturally, following the interviewees lead as they take in the information.

Step 4: Brief Intro to Types of Organizational Values

- Preparing them to discern Core Values.

\footnotetext{
${ }^{20}$ If applicable. See Appendix J, Figure J.2 for template.
}

${ }^{21} \mathrm{~A}$ reminder to the researcher to smile and be aware of body language and tone of voice during the interview. 
Step 5: Core Values Candidate List

- Review Sheet

- Ask Interviewee "What is missing from this Candidates List?"

- Ask Interviewee to Identify their perceived Core Value Candidates

- Ask Interviewee Rank \& Weight (prioritize) Value-Candidates on this sheet

Step 6: Custom Core Value - Behavior Association \& Ranking Form

- Follow Prompts on Page

- Rank behaviors for inclusion in the final product.

Step 7: Language Probe (Email Follow-up is optional).

- Probe for Language/Format Suggestions on the final product (E.g., How long/short?)

- Let them know you will email the Core Value Candidates to interviewees for language suggestions to be done in peace/private.

- Set a 'due' date.

Step 8: Thank you! You did awesome!

\section{Overview:}

The purpose of the second interview is to guide interviewees through a process of:

6) Familiarizing the interviewee with the Competing Values Framework

7) Sensitizing the interviewee to descriptions, perspectives and data points reflecting their organizational culture and values

8) Identifying organizational core values (from the interviewees perspective)

9) Associating behaviors with each identified core value.

10) Discriminating between core and other types of organizational values (if time).

The end result of this interview process will be, for each interviewee, a set of interviewee-identified core values the company, a set of behaviors the interviewee associates with each identified core value, and a rich conversation for further transcription, coding and analysis by the researcher. 
Appendix J: Overall Culture Assessment Template

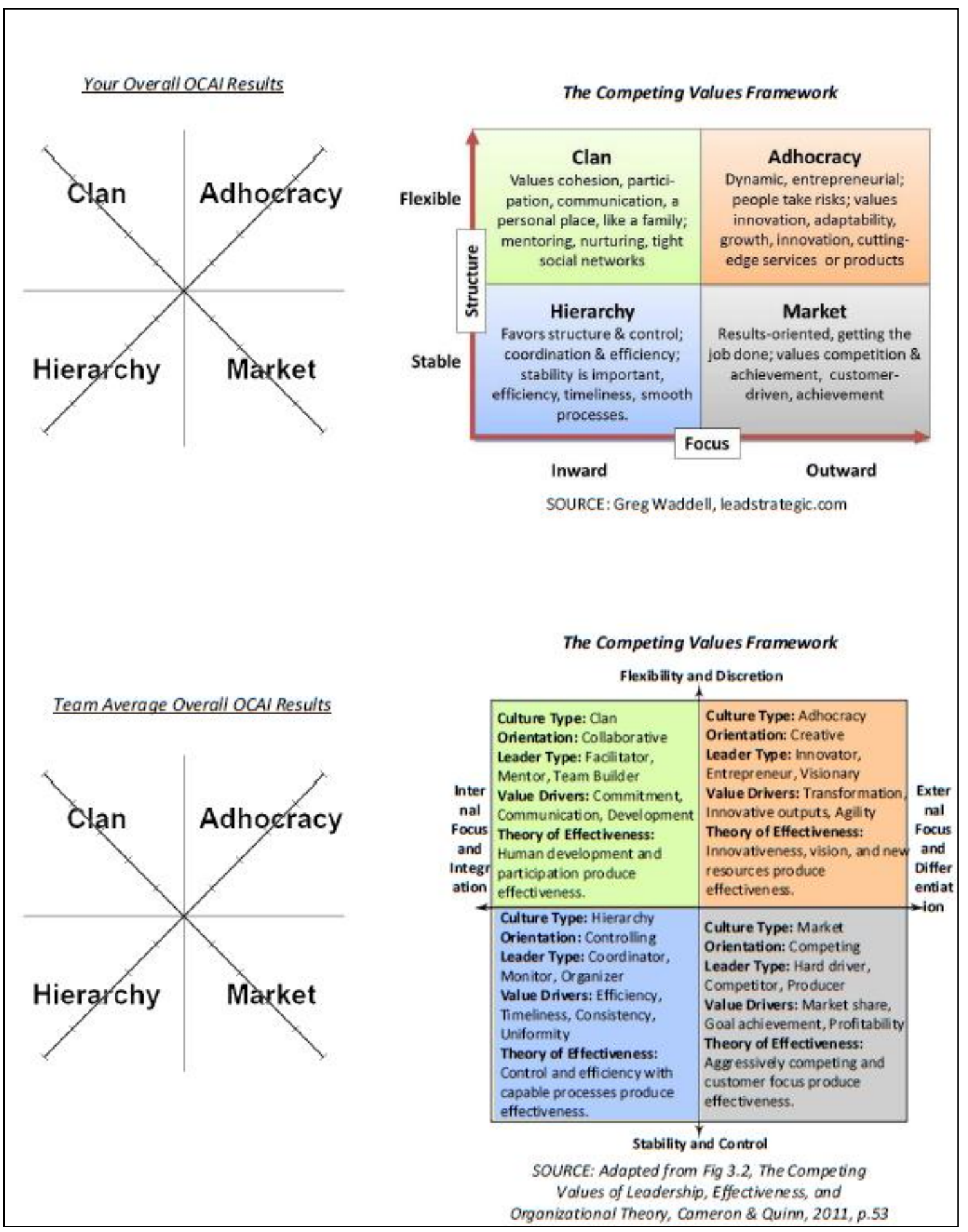

Figure 29: Overall Culture Assessment Results Page Template for Second Round Interviews. 


\section{Appendix K: Itemized Culture Assessment Template}

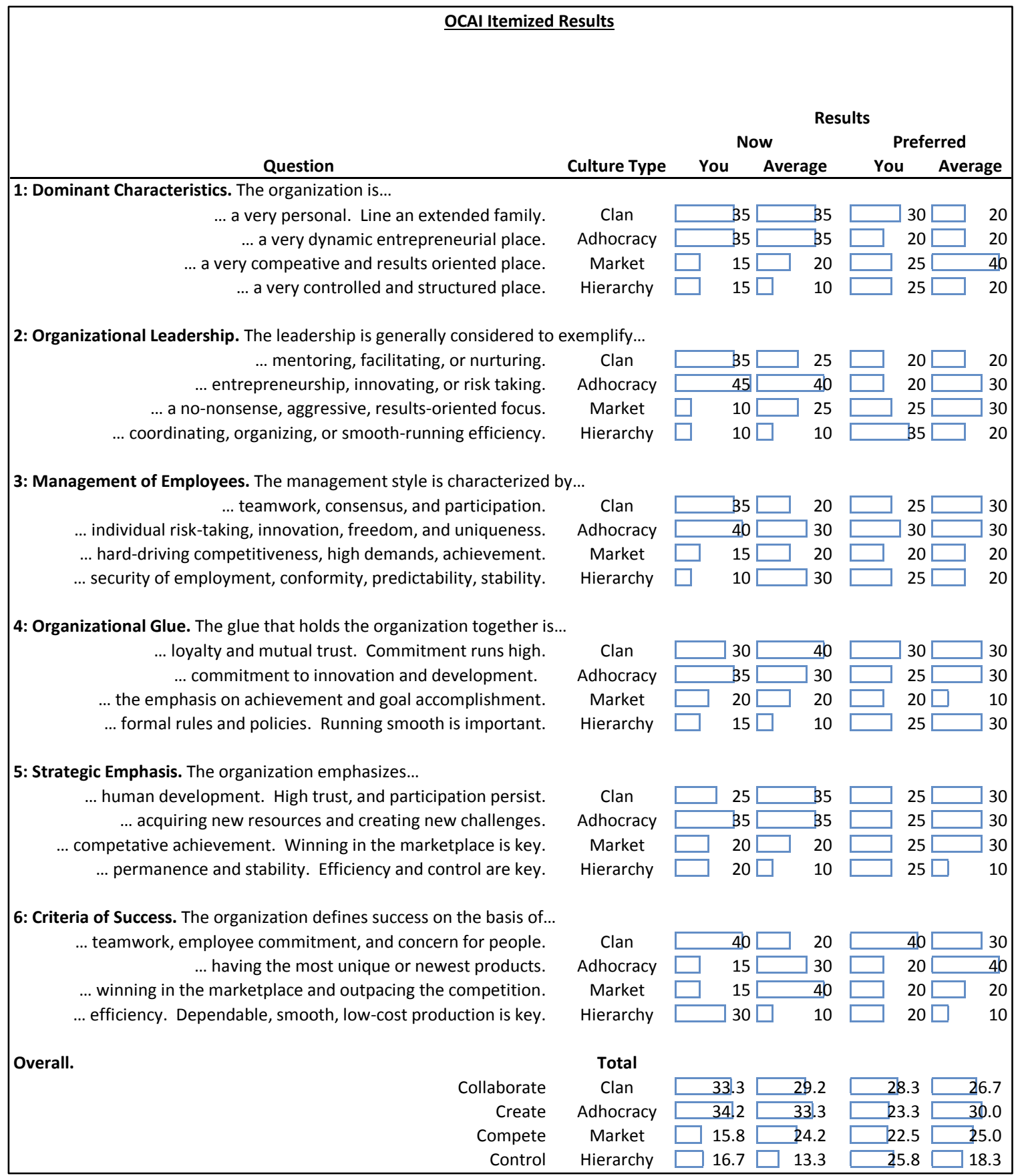

Figure 30: Itemized Culture Assessment Results Page Template for Second Round Interviews. 


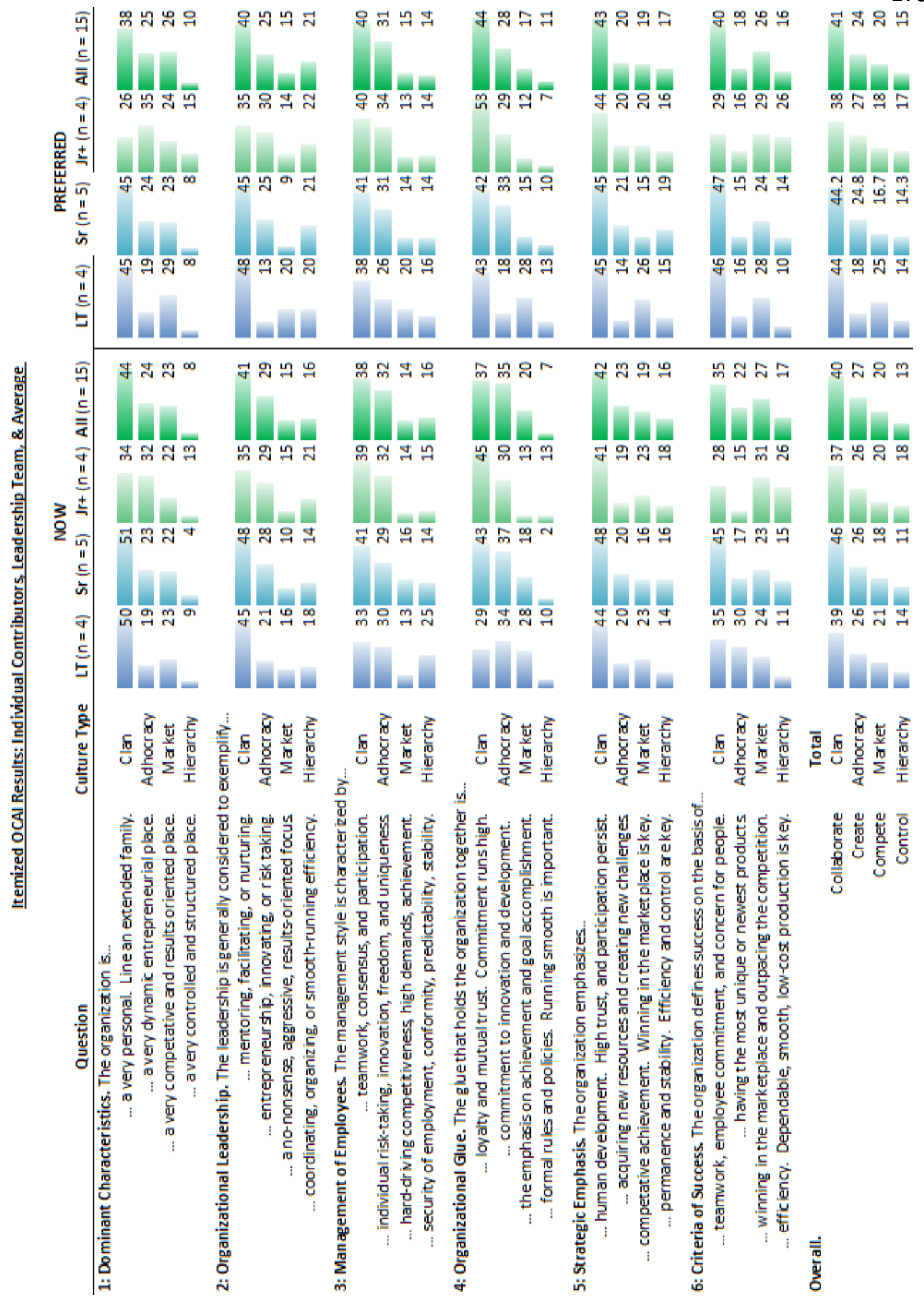

Figure 31: Optional Itemized Results Page Template for Multiple Employee Groups. Figure as discussed in Chapter 6.4. 
Appendix L: Types of Organizational Values Chart

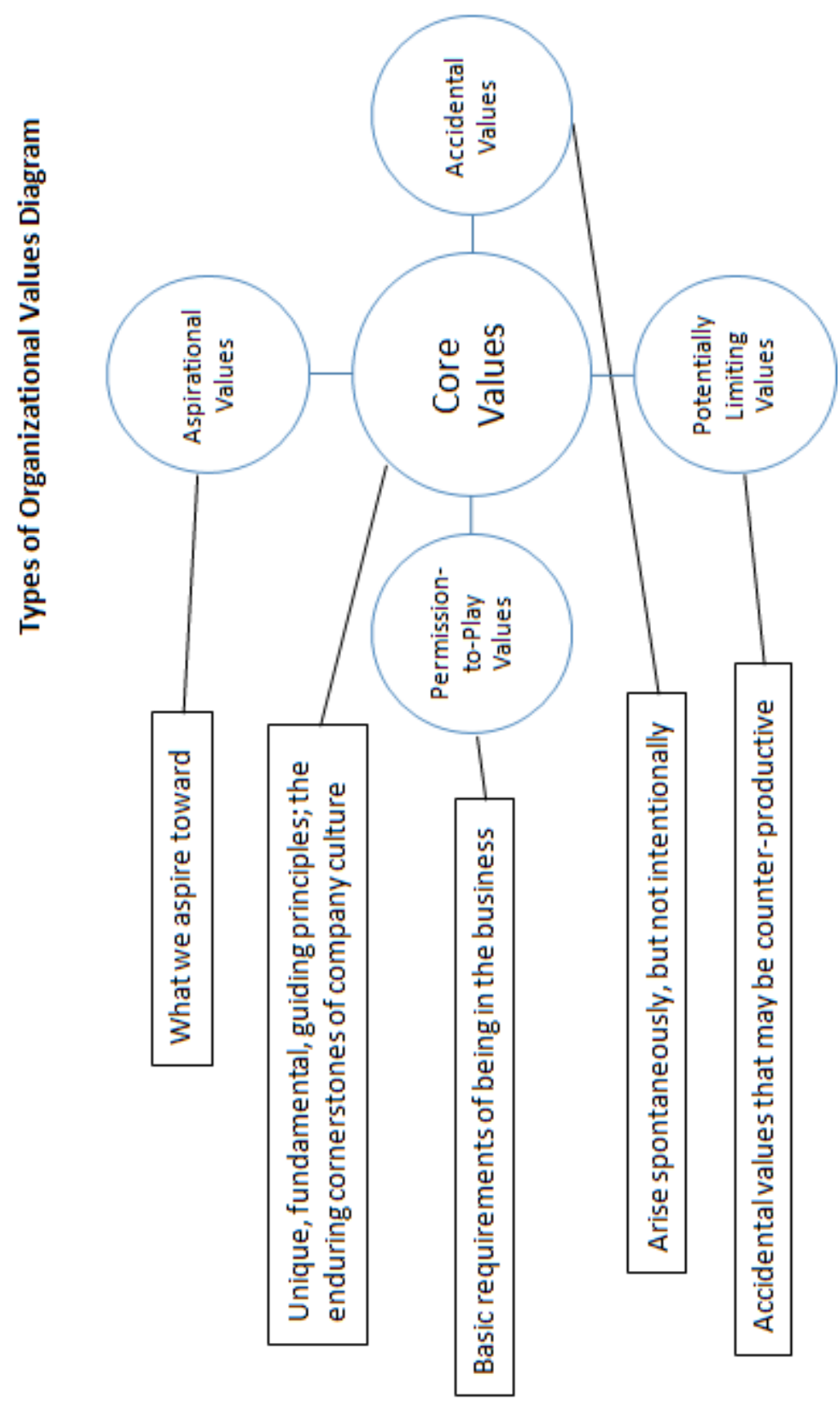

Figure 32: Types of Organizational Values Chart. 


\section{Appendix M: Core Values Identification Form (Example)}

This is a list of values based on the interviews, observations, and document review at [company name].

1. Add any important values that are missing.

2. Rank the top, most important values at [company name] in order of perceived importance, or impact on organizational effectiveness, with 1 being the most important. Please rank at least 5.

Servant's Heart: Service is at our core. We all enjoy knowing that our work is a benefit and delight to the next person downstream, whether that's another employee, another team, or the final customer. We don't aim to satisfice; we aim to exceed expectations.

Personal Growth: We have a lot of really talented employees. Really talented employees. This is because we are all learners. We believe that the willingness and ability to learn is more valuable than prior experience. If we are not advancing we are falling behind.

Problem Solving Prowess: What we do is solve problems for customers. And we love new challenges. We get bored easily. That's why we enjoy taking on jobs that others avoid or fail.

A Culture of Integrity, Maturity \& Respectfulness: Having a healthy culture allows us to thrive. We believe everything we do should be done well. We treat our customers, each other, and our community with respect.

Do What You Love: We are engineers, pioneers, and craftsmen. Some of us love solving new problems every day. Some of us love building those solutions.

Humble Talent: Our success depends on our ability to learn, adapt, and solve new problems. When talent loses humility, it loses those abilities. We believe overconfidence creates incompetence 


\section{Appendix N: Value-Behavior Association Form (Example)}

Core values should provide a clear blueprint for employee behavior.

1. Review the trends in value candidates. Include any important ideas that are missing. 2. Within each value category, rank the top, most important behaviors at [COMPANY], in order of perceived importance (or impact on organizational effectiveness) with 1 being the most important. Please rank at least 4 in each category.

3. (Optional) Weight each of your ranked values and/or behaviors within categories. The total must equal $100 \%$

\section{Major Trends:}

Weight: Ranking:

\begin{tabular}{|l|l|}
\hline & \\
\hline & \\
\hline & \\
\hline & \\
\hline & \\
\hline & \\
\hline & \\
\hline & \\
\hline & \\
\hline & \\
\hline & \\
\hline & \\
\hline & \\
\hline & \\
\hline & \\
\hline & \\
\hline & \\
\hline & \\
\hline
\end{tabular}

The [COMPANY] Winning Mindset: Winning. Learning. Leading. Together. We succeed through teamwork, general awesomeness, and great strategy.

Committed to extremely high quality results. Demonstrate personal accountability for results and take pride in their work. Driven. Results-oriented, self-motivated, competitive in a good way.

Your "standard overachiever." Holds themselves to high personal standards. Desires to go above and beyond.

Think like winners. Strategic, competitive; always seeking better ways to do the best possible job.

Relentless pursuit of Personal Development. A desire to learn and develop new skills, because our industry changes so quickly. Healthy Competition. Competing 'together' rather than 'against each other'.

Developing others. Building others up \& caring about the success of coworkers.

Leadership. We want all of our employees to be leaders. It is up to each person to advance.

Team Mentality. We're all in this together. Concern for other's success: clients, co-workers, and the [COMPANY] family Ownership Mentality. Invested in the success client accounts and the company as a whole.

Passionate. 


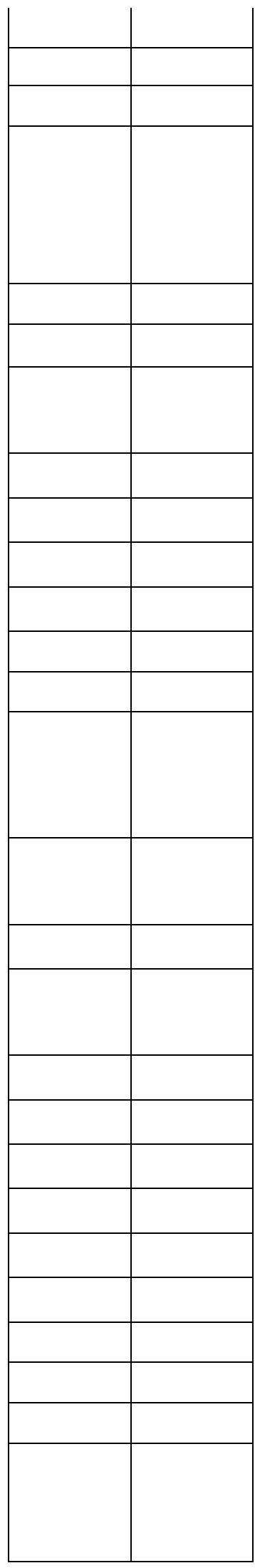

Balanced Analytical \& Emotional Intelligence. Technical Prowess. People Skills. Balance. Our work requires a balance of both technical prowess and people skills. Both are necessary, in balance.

\section{Technical Fit \& Flexibility.}

Analytical intelligence. Able to sift through a lot of data and produce insight.

Problem Solving and Troubleshooting skills.

Able to learn and re-learn the fast changing industry we are in.

Willingness to adapt every day as the world changes.

Eagerness to learn. Things are changing constantly.

Character \& People Skills. We look for wholesomeness in

hiring. We look for people we would want to spend the next 10 or 15 years with, every day.

Integrity. We are performance based, we don't lock our clients into contracts.

Authenticity, Transparency, Being real.

Have faith in people. Default to a positive outlook on others; clients and each other.

Easy to talk to. Approachable.

Good communication.

Owning up to mistakes.

Positive \& Respectful.

Active listening.

Work ethic

Caring for others. Caring is at our core. We exist because we serve our clients extremely well. We understand that serving others also fulfills the servant, so we focus on others rather than 


\begin{tabular}{|l|l|} 
& \\
\hline & \\
\hline & \\
\hline & \\
\hline & \\
\hline & \\
\hline & \\
\hline & \\
\hline & \\
\hline & \\
\hline
\end{tabular}

ourselves.

Passionate about serving the clients extremely well. We focus explicitly on 'does our client win?'

Passion about what we do. Always seeking better results.

Compassion is key at [COMPANY]. Generosity fulfills the giver, and we get that.

Passionate about serving each other and helping each other succeed, personally and professionally. 


\section{Appendix 0: Company-Specific Data Tables}

Appendix $\mathrm{O}$ is a technical appendix for the company-specific results discussed in

Chapter 5. For ease of use, the titles and numbering of the data tables in this appendix reflect the titles and numbers of the Chapter 5 sections where these tables are referenced.

Table 4: Company A: Delivering Results. Tracing Core Value "Delivering Results" through constituent conceptual components and back to collected data at Company A. Underlined text is raw data, with context described parenthetically in italics.

\begin{tabular}{|c|c|c|}
\hline Dominant Themes: & Examples of Supporting Data: & Data Source: \\
\hline \multirow[t]{5}{*}{ Delivering Results } & $\begin{array}{l}\text { Well, at the end of the day, delivering results is probably } \\
\text { number one. I mean, you don't stay in business if you } \\
\text { don't deliver. (Discussing the relative importance of } \\
\text { various company values). }\end{array}$ & $\begin{array}{l}\text { Interview with } \\
\text { [Employee A] }\end{array}$ \\
\hline & $\begin{array}{l}\text { Achieve a task then are looking for more. Task Achiever. } \\
\text { ([Employee C]). (Written responses by Interviewees in } \\
\text { response to the following prompt: "Tell me about your } \\
\text { star employees. Tell me about the people who represent } \\
\text { the best of your organization." Written on the Interview } 1 \\
\text { Worksheet) }\end{array}$ & $\begin{array}{l}\text { Interview } 1 \\
\text { Worksheet }\end{array}$ \\
\hline & $\begin{array}{l}\text { really understand that we're a small company and we're } \\
\text { really gonna expect you to perform your job." (In } \\
\text { response to the question: "Tell me, what is one piece of } \\
\text { advice you would give to a new employee or applicant } \\
\text { about how to thrive at this company?") }\end{array}$ & $\begin{array}{l}\text { Interview with } \\
\text { [Employee B] }\end{array}$ \\
\hline & $\begin{array}{l}\text { Well without results you don't have anything. That's } \\
\text { probably } 90 \% \text { of it is delivering results. }\end{array}$ & $\begin{array}{l}\text { Interview 2, } \\
\text { [Employee A] }\end{array}$ \\
\hline & $\begin{array}{l}\text { Results. (Part of a pre-existing, longer statement of } \\
\text { company values.) }\end{array}$ & $\begin{array}{l}\text { Document } \\
\text { Review, Original } \\
\text { Values } \\
\text { Statement }\end{array}$ \\
\hline
\end{tabular}




\begin{tabular}{|c|c|c|}
\hline \multirow[t]{5}{*}{$\begin{array}{l}\text { We strive to } \\
\text { achieve excellent } \\
\text { results. }\end{array}$} & $\begin{array}{l}\text { The end result of a roof (he said this without hesitation). } \\
\text { I mean that's what we do. And when you walk and it } \\
\text { turns out, it's skin tight and everything, all the walk pad } \\
\text { around the units, everything stands out and looks really } \\
\text { good, you know, that's why we're all here. (In response to } \\
\text { the question, 'What would you say is the best part about } \\
\text { working here that someone like me might not be able to } \\
\text { figure out by spending a week here observing or asking } \\
\text { good questions - what's something that I might miss?') }\end{array}$ & $\begin{array}{l}\text { Interview with } \\
\text { [Employee D] }\end{array}$ \\
\hline & $\begin{array}{l}\text { The thing I try to do with myself and let everybody know } \\
\text { is we want to make the best decisions. It's not about me } \\
\text { winning or you winning, it's about 'what's the best } \\
\text { decision?'. (In response to the question: "Tell me, what is } \\
\text { one piece of advice you would give to a new employee or } \\
\text { applicant about how to thrive at this company?") }\end{array}$ & $\begin{array}{l}\text { Interview with } \\
\text { [Employee A] }\end{array}$ \\
\hline & $\begin{array}{l}\text { Delight in a job well done. Deep passion. ([Employee C]). } \\
\text { (Written responses by Interviewees in response to the } \\
\text { following prompt: "Tell me about your star employees. } \\
\text { Tell me about the people who represent the best of your } \\
\text { organization." Written on the Interview } 1 \text { Worksheet) }\end{array}$ & $\begin{array}{l}\text { Interview } 1 \\
\text { Worksheet }\end{array}$ \\
\hline & $\begin{array}{l}\text { Well, you know the, the fulfillment and joy that you get } \\
\text { after you learn more about roofs here so you can put } \\
\text { down a quality product. And you know that it's going to } \\
\text { last a long time, should be in there for } 20 \text { years, you } \\
\text { know? You put that roof on there, it's gonna hand around } \\
\text { awhile. And completion of that, 'yea, we did that, we did } \\
\text { that work, yea, and that's gonna hang up there a long } \\
\text { time'. (In response to the question, 'What would you say is } \\
\text { the best part about working here that someone like me } \\
\text { might not be able to figure out by spending a week here } \\
\text { observing or asking good questions - what's something } \\
\text { that I might miss?') }\end{array}$ & $\begin{array}{l}\text { Interview with } \\
\text { [Employee C] }\end{array}$ \\
\hline & $\begin{array}{l}\text { Results - Measured progress. We strive to achieve } \\
\text { excellent results. (Part of a pre-existing, longer statement } \\
\text { of company values.) }\end{array}$ & $\begin{array}{l}\text { Document } \\
\text { Review } \\
\text { Original Values } \\
\text { Statement }\end{array}$ \\
\hline $\begin{array}{l}\text { Bringing a } \\
\text { positive, can-do } \\
\text { attitude to every } \\
\text { challenge is the } \\
\text { key to our success. }\end{array}$ & $\begin{array}{l}\text { It's all about having a good attitude. That's the main } \\
\text { thing, attitude on a roof. Like, you can change the whole } \\
\text { moral with one bad attitude. The whole crew slows } \\
\text { down, so a lot of it is attitude. (In response to the } \\
\text { question: "Tell me, what is one piece of advice you would } \\
\text { give to a new employee or applicant about how to thrive } \\
\text { at this company?") }\end{array}$ & $\begin{array}{l}\text { Interview with } \\
\text { [Employee D] }\end{array}$ \\
\hline
\end{tabular}




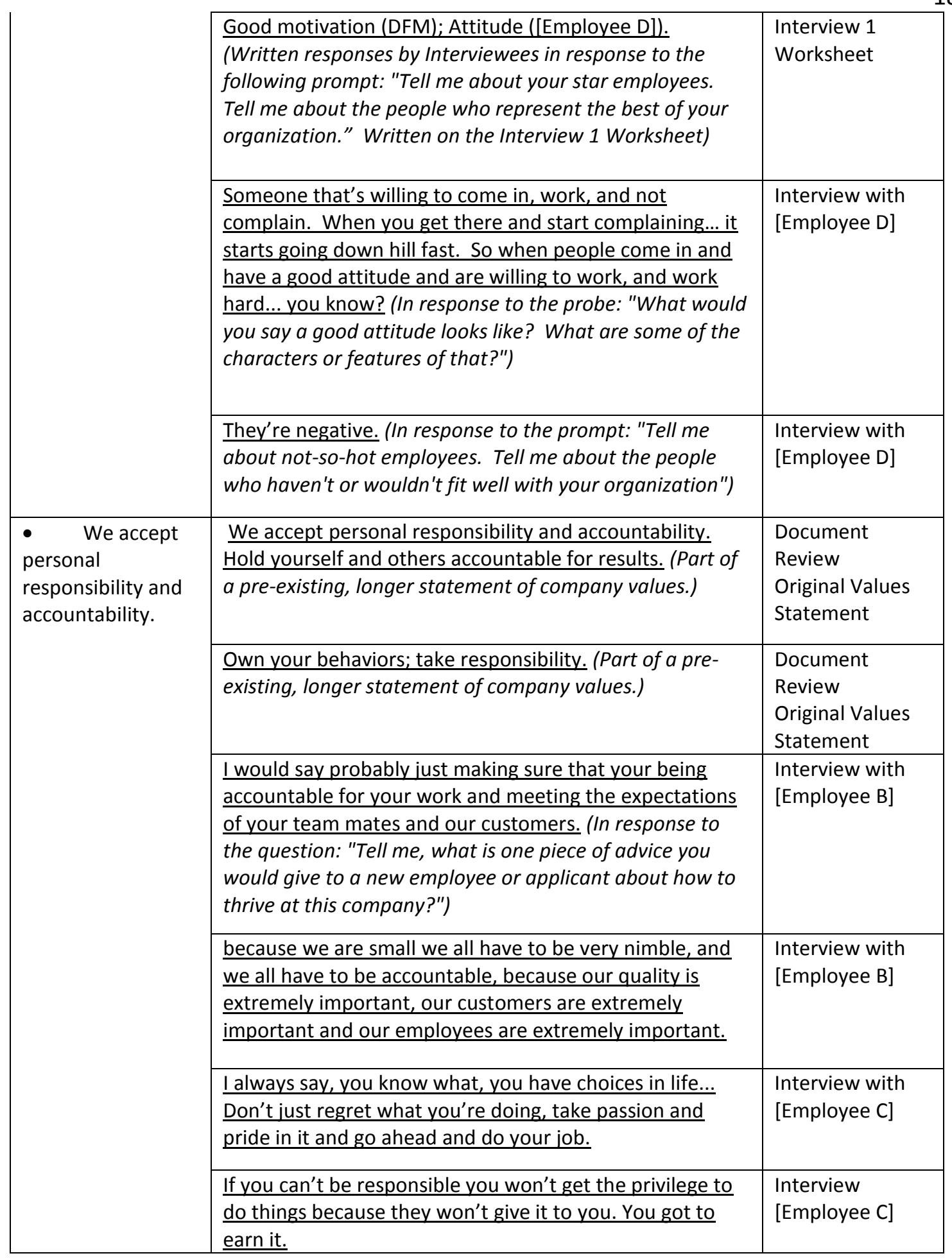




\begin{tabular}{|c|c|c|}
\hline \multirow[t]{3}{*}{$\begin{array}{l}\text { - We work } \\
\text { hard \& smart. }\end{array}$} & $\begin{array}{l}\text { Someone that's willing to come in, work, and not } \\
\text { complain. When you get there and start complaining... it } \\
\text { starts going downhill fast. So when people come in and } \\
\text { have a good attitude and are willing to work, and work } \\
\text { hard... you know? (In response to the probe: "What would } \\
\text { you say a good attitude looks like? What are some of the } \\
\text { characters or features of that?") }\end{array}$ & $\begin{array}{l}\text { Interview with } \\
\text { [Employee D] }\end{array}$ \\
\hline & $\begin{array}{l}\text { Hard work ([Employee C]); Always being productive } \\
\text { ([Employee D]]; Good people identify root causes of } \\
\text { problems ([Employee A]). (Written responses by } \\
\text { Interviewees in response to the following prompt: "Tell me } \\
\text { about your star employees. Tell me about the people who } \\
\text { represent the best of your organization." Written on the } \\
\text { Interview } 1 \text { Worksheet) }\end{array}$ & $\begin{array}{l}\text { Interview } 1 \\
\text { Worksheet }\end{array}$ \\
\hline & $\begin{array}{l}\text { the guys who don't try to take hour breaks, go right back } \\
\text { to work, always stay busy. If you run into something you } \\
\text { don't quite get or understand you go over and clean up } \\
\text { trash until someone has time to teach you, you know, } \\
\text { just, constantly trying to do something productive. (In } \\
\text { response to the prompt: "Tell me about your star } \\
\text { employees. Tell me about the people who represent the } \\
\text { best }\end{array}$ & $\begin{array}{l}\text { Interview with } \\
\text { [Employee D] }\end{array}$ \\
\hline
\end{tabular}


Table 5: Company A: Integrity. Tracing Core Value "Integrity" through constituent conceptual components and back to collected data at Company A during the initial data collection phase. Underlined text is raw data, with context described parenthetically in italics.

\begin{tabular}{|c|c|c|}
\hline $\begin{array}{l}\text { Conceptual } \\
\text { Components: }\end{array}$ & Examples of Supporting Data: & Data Source: \\
\hline \multirow[t]{3}{*}{$\begin{array}{l}\text { Integrity. Honesty } \\
\text { in all we do. }\end{array}$} & $\begin{array}{l}\text { Integrity - Honesty in all we do. (The first value listed on } \\
\text { a pre-existing, longer statement of company values.) }\end{array}$ & $\begin{array}{l}\text { Document } \\
\text { Review } \\
\text { Original Values } \\
\text { Statement }\end{array}$ \\
\hline & $\begin{array}{l}\text { I guess we always expect our guys to be honest with us, } \\
\text { whether it's good or bad. You know, we want em to, } \\
\text { you know, being honest, that's a big part of it. Because } \\
\text { if you mess up something and you're honest about it we } \\
\text { get it fixed vs. monkeying around and trying to figure out } \\
\text { who did what. (In response to the question: "Is there } \\
\text { anything I'm missing or not asking about correctly that } \\
\text { you would think is important for me to understand?") }\end{array}$ & $\begin{array}{l}\text { Interview with } \\
\text { [Employee D] }\end{array}$ \\
\hline & $\begin{array}{l}\text { I always say that with privilege is the responsibility of } \\
\text { privilege, the responsibility of privilege is absolute } \\
\text { integrity that you give to the company. }\end{array}$ & $\begin{array}{l}\text { Interview with } \\
\text { [Employee C] }\end{array}$ \\
\hline \multirow[t]{2}{*}{$\begin{array}{l}\text { - We must } \\
\text { always walk the } \\
\text { talk, own our } \\
\text { actions and take } \\
\text { responsibility. }\end{array}$} & $\begin{array}{l}\text { Walk the talk; demonstrate consistency between your } \\
\text { actions and your words. (Part of a pre-existing, longer } \\
\text { statement of company values.) }\end{array}$ & $\begin{array}{l}\text { Document } \\
\text { Review } \\
\text { Original Values } \\
\text { Statement }\end{array}$ \\
\hline & $\begin{array}{l}\text { Got to walk the talk ([Employee A]). (Written responses } \\
\text { by Interviewees in response to the following prompt: } \\
\text { "Tell me about your star employees. Tell me about the } \\
\text { people who represent the best of your organization." } \\
\text { Written on the Interview } 1 \text { Worksheet) }\end{array}$ & $\begin{array}{l}\text { Interview } 1 \\
\text { Worksheet }\end{array}$ \\
\hline \multirow[t]{2}{*}{$\begin{array}{l}\text { Do work to } \\
\text { the best of your } \\
\text { ability and to the } \\
\text { highest quality. }\end{array}$} & $\begin{array}{l}\text {...bad workmanship. Just sloppy. (In response to the } \\
\text { prompt: "Tell me about not-so-hot employees. Tell me } \\
\text { about the people who haven't or wouldn't fit well with } \\
\text { your organization") }\end{array}$ & $\begin{array}{l}\text { Interview with } \\
\text { [Employee D] }\end{array}$ \\
\hline & $\begin{array}{l}\text { And it's kind of a game, between foremen, }, . . \text { who can get } \\
\text { the jobs in, under [budgeted man-hours], the lowest, but } \\
\text { still have them perfect. }\end{array}$ & $\begin{array}{l}\text { Interview with } \\
\text { [Employee D] }\end{array}$ \\
\hline \multirow[t]{2}{*}{$\begin{array}{l}\text { - We don't } \\
\text { take shortcuts. We } \\
\text { do it the right way. }\end{array}$} & $\begin{array}{l}\text { Do the job right the first time. (Part of a pre-existing, } \\
\text { longer statement of company values.) }\end{array}$ & $\begin{array}{l}\text { Document } \\
\text { Review } \\
\text { Original Values } \\
\text { Statement }\end{array}$ \\
\hline & $\begin{array}{l}\text {...your main goal here is to become a roofer. If you're } \\
\text { already an experienced roofer, wipe away all your bad } \\
\text { habits. You know, start doing it right. }\end{array}$ & $\begin{array}{l}\text { Interview with } \\
\text { [Employee C] }\end{array}$ \\
\hline
\end{tabular}


Table 6: Company A: Developing our People. Tracing the Core Value "Developing our People" through constituent conceptual components and back to data collected during the initial data collection phase at Company A. Underlined text is raw data, with context described parenthetically in italics.

\begin{tabular}{|c|c|c|}
\hline $\begin{array}{l}\text { Conceptual } \\
\text { Components: }\end{array}$ & Examples of Supporting Data: & Data Source: \\
\hline $\begin{array}{l}\text { Developing our } \\
\text { People }\end{array}$ & $\begin{array}{l}\text { Developing our People (Phrase derived directly from CVF } \\
\text { clan quadrant descriptions in acknowledgement of the } \\
\text { desired decrease in market culture based on OCAl results. } \\
\text { Validated in Interview } 3 \text { and after presentation). }\end{array}$ & $\begin{array}{l}\text { Theorization, } \\
\text { CVF Text. }\end{array}$ \\
\hline \multirow{2}{*}{$\begin{array}{l}\text { We are all } \\
\text { craftsmen, } \\
\text { mentors and } \\
\text { teammates. We } \\
\text { help each other } \\
\text { improve every day. } \\
\text { We do this because } \\
\text { we honestly care } \\
\text { about everyone's } \\
\text { personal growth } \\
\text { and it makes us } \\
\text { more effective. }\end{array}$} & $\begin{array}{l}\text { Everybody works together. That's a big one. They help } \\
\text { you to help them, you know? They help you if you don't } \\
\text { understand something and we really all work together } \\
\text { really well. Everyone in the field, they're all really good } \\
\text { with their guys, they have a close relationship, they're } \\
\text { easy, for the most part, easy to work with. }\end{array}$ & $\begin{array}{l}\text { Interview with } \\
\text { [Employee D] }\end{array}$ \\
\hline & $\begin{array}{l}\text { If you can't be responsible you won't get the privilege to } \\
\text { do things because they won't give it to you. You got to } \\
\text { earn it. Hard work and keep your mind open to new ways } \\
\text { to help everyone. Team players here. }\end{array}$ & $\begin{array}{l}\text { Interview with } \\
\text { [Employee C] }\end{array}$ \\
\hline \multirow[t]{2}{*}{$\begin{array}{l}\text { - Be willing to } \\
\text { listen and learn. }\end{array}$} & $\begin{array}{l}\text { Just be willing to learn. I mean, that's the main thing } \\
\text { [besides attitude]. (In response to the question: "Tell me, } \\
\text { what is one piece of advice you would give to a new } \\
\text { employee or applicant about how to thrive at this } \\
\text { company?") }\end{array}$ & $\begin{array}{l}\text { Interview with } \\
\text { [Employee D] }\end{array}$ \\
\hline & $\begin{array}{l}\text { Willing to learn ([Employee D]); Strong Listening } \\
\text { ([Employee C]); Totally want to be in the learning mode } \\
\text { and learn everything about [industry]. ([Employee C]); } \\
\text { Listen to what more experienced folks share ([Employee } \\
\text { C]). (Written responses by Interviewees in response to the } \\
\text { following prompt: "Tell me about your star employees. } \\
\text { Tell me about the people who represent the best of your } \\
\text { organization." Written on the Interview } 1 \text { Worksheet) }\end{array}$ & $\begin{array}{l}\text { Interview } 1 \\
\text { Worksheet }\end{array}$ \\
\hline \multirow[t]{4}{*}{$\begin{array}{l}\text { - Help and } \\
\text { train each other. }\end{array}$} & $\begin{array}{l}\text { We'll train them... we'll push them towards sheet metal } \\
\text { or service or there's always other spots we can make } \\
\text { them excel. So as long as they're willing to learn and get } \\
\text { better, we're willing to put them in the right spot. }\end{array}$ & $\begin{array}{l}\text { Interview with } \\
\text { [Employee D] }\end{array}$ \\
\hline & $\begin{array}{l}\text { We are a training company and we will train you from the } \\
\text { day you start. }\end{array}$ & $\begin{array}{l}\text { Interview with } \\
\text { [Employee C] }\end{array}$ \\
\hline & $\begin{array}{l}\text { Come in here with a strong positive attitude and see if } \\
\text { you got some experience that you have to bring to the } \\
\text { table to help them... (Advice for a new employee or } \\
\text { leader). }\end{array}$ & $\begin{array}{l}\text { Interview with } \\
\text { [Employee C] }\end{array}$ \\
\hline & $\begin{array}{l}\text { build others up. (Part of a pre-existing, longer statement } \\
\text { of company values.) }\end{array}$ & $\begin{array}{l}\text { Document } \\
\text { Review, } \\
\text { Original Values } \\
\text { Statement }\end{array}$ \\
\hline
\end{tabular}


- Ask

questions.
Ask questions ([Employee B]). (Written responses by Interviewees in response to the following prompt: "Tell me about your star employees. Tell me about the people who represent the best of your organization." Written on the Interview 1 Worksheet)

If you don't know how to do something, ask for help. (Part of a pre-existing, longer statement of company values.)
Interview 1

Worksheet

Document

Review, Original Values

Statement 
Table 7: Company B: Do What You Love. Tracing Core Value "Do What You Love" through constituent conceptual components and back to collected data at Company B. Underlined text is raw, collected data, with context described parenthetically in italics.

\begin{tabular}{|c|c|c|}
\hline Dominant Themes: & Examples of Supporting Data: & Data Source: \\
\hline \multirow{3}{*}{$\begin{array}{l}\text { Do What You Love: We } \\
\text { are engineers, } \\
\text { pioneers, and } \\
\text { craftsmen. Some of us } \\
\text { love solving new } \\
\text { problems every day. } \\
\text { Some of us love } \\
\text { building those } \\
\text { solutions. Some love to } \\
\text { facilitate the smooth } \\
\text { operation of all our } \\
\text { activities. Whether it is } \\
\text { the challenge, the } \\
\text { aesthetic, or seeing the } \\
\text { end result in the } \\
\text { finished product, we } \\
\text { love what we do and } \\
\text { have fun doing it. }\end{array}$} & $\begin{array}{l}\text { Loves what they do, not here for the paycheck } \\
\text { ([Employee A]); Subject Matter Expertise ([Employee } \\
\text { C]); Embracing a challenge ([Employee B]]; (Written } \\
\text { responses by Interviewees in response to the following } \\
\text { prompt: "Tell me about your star employees. Tell me } \\
\text { about the people who represent the best of your } \\
\text { organization." Written on the Interview } 1 \text { Worksheet) }\end{array}$ & $\begin{array}{l}\text { Interview } 1 \\
\text { Worksheet }\end{array}$ \\
\hline & $\begin{array}{l}\text { Three essential groups of employees exist at Company } \\
\text { B: engineers who design systems for construction, } \\
\text { workshop employees who build those systems, and the } \\
\text { professional support employees who create and } \\
\text { coordinate business processes. }\end{array}$ & $\begin{array}{l}\text { Field } \\
\text { Observation }\end{array}$ \\
\hline & $\begin{array}{l}\text { Loves what they do; not here for a paycheck. (Written } \\
\text { response by interviewee after the instruction prompt } \\
\text { to add any important language or behaviors missing } \\
\text { from the core values identification exercise) }\end{array}$ & $\begin{array}{l}\text { Value- } \\
\text { Behavior } \\
\text { Association } \\
\text { Worksheet, } \\
\text { [Employee B] }\end{array}$ \\
\hline \multirow[t]{3}{*}{$\begin{array}{l}\text { Focus on your } \\
\text { gifts and talents: use } \\
\text { and develop them. }\end{array}$} & $\begin{array}{l}\text { Not afraid to dig in, learn, and ask questions } \\
\text { ([Employee E]); Be a learner ([Employee B]]). (Written } \\
\text { responses by Interviewees in response to the following } \\
\text { prompt: "Tell me about your star employees. Tell me } \\
\text { about the people who represent the best of your } \\
\text { organization." Written on the Interview } 1 \text { Worksheet) }\end{array}$ & $\begin{array}{l}\text { Interview } 1 \\
\text { Worksheet }\end{array}$ \\
\hline & $\begin{array}{l}\text { a big thing as a core value is to be a learner. Learn } \\
\text { how to learn your craft... learn how to assimilate } \\
\text { information that you're gonna need. Because to be a } \\
\text { self-starter and initiator will really really help your } \\
\text { career here. That's a highly valued trait. (In response } \\
\text { to the question: "Tell me, what is one piece of advice } \\
\text { you would give to a new employee or applicant about } \\
\text { how to thrive at this company?") }\end{array}$ & $\begin{array}{l}\text { Interview with } \\
\text { [Employee B] }\end{array}$ \\
\hline & $\begin{array}{l}\text { As l've watched over my } 8 \text { years here, different people } \\
\text { come and thrive, it's been the ones that have learned } \\
\text { how to learn. }\end{array}$ & $\begin{array}{l}\text { Interview with } \\
\text { [Employee B] }\end{array}$ \\
\hline $\begin{array}{l}\text { Enjoy solving } \\
\text { daily challenges. } \\
\text { Embrace them with a } \\
\text { good attitude. }\end{array}$ & $\begin{array}{l}\text { Problem solver mentality ([Employee C]); Stick to it } \\
\text { until problem solved ([Employee E]); Diligence. } \\
\text { Embracing a challenge. ([Employee B]). (Written } \\
\text { responses by Interviewees in response to the following } \\
\text { prompt: "Tell me about your star employees. Tell me } \\
\text { about the people who represent the best of your } \\
\text { organization." Written on the Interview } 1 \text { Worksheet) }\end{array}$ & $\begin{array}{l}\text { Interview } 1 \\
\text { Worksheet }\end{array}$ \\
\hline
\end{tabular}




\begin{tabular}{|c|c|c|}
\hline & $\begin{array}{l}\text { You're called upon to really really own the projects } \\
\text { you work on, and you can't let up. You gotta stay at it, } \\
\text { even though it's really hard. And somehow you have } \\
\text { to learn to enjoy being in the trenches that are hard } \\
\text { like that, and that's not an easy path for most people I } \\
\text { don't think. }\end{array}$ & $\begin{array}{l}\text { Interview with } \\
\text { [Employee B] }\end{array}$ \\
\hline & $\begin{array}{l}\text { challenged daily. (Written response by interviewee } \\
\text { after the instruction prompt to add any important } \\
\text { language or behaviors missing from the core values } \\
\text { identification exercise) }\end{array}$ & $\begin{array}{l}\text { Value- } \\
\text { Behavior } \\
\text { Association } \\
\text { Worksheet, } \\
\text { [Employee E] }\end{array}$ \\
\hline \multirow[t]{2}{*}{$\begin{array}{l}\text { - Stay focused on } \\
\text { delivering great results. } \\
\text { Everything else will } \\
\text { follow. }\end{array}$} & $\begin{array}{l}\text { Always looking for improvement opportunities } \\
\text { ([Employee E]]; Diligence ([Employee B]). (Written } \\
\text { responses by Interviewees in response to the following } \\
\text { prompt: "Tell me about your star employees. Tell me } \\
\text { about the people who represent the best of your } \\
\text { organization." Written on the Interview } 1 \text { Worksheet) }\end{array}$ & $\begin{array}{l}\text { Interview } 1 \\
\text { Worksheet }\end{array}$ \\
\hline & $\begin{array}{l}\text { Look for ways to improve current status. (Written } \\
\text { response by interviewee after the instruction prompt } \\
\text { to add any important language or behaviors missing } \\
\text { from the core values identification exercise) }\end{array}$ & $\begin{array}{l}\text { Value- } \\
\text { Behavior } \\
\text { Association } \\
\text { Worksheet, } \\
\text { [Employee E] }\end{array}$ \\
\hline \multirow[t]{3}{*}{$\begin{array}{l}\text { Embrace your } \\
\text { role. If you're not } \\
\text { thriving, let's find a } \\
\text { place where you will. }\end{array}$} & $\begin{array}{l}\text { Loves what they do, not here for the paycheck } \\
\text { ([Employee A]]; Always looking for improvement } \\
\text { opportunities ([Employee E]); Putting people where } \\
\text { they thrive ([Employee C] LT); Getting people in } \\
\text { positions that match their talents/passion ([Employee } \\
\text { A] LT); Own your progress. Love the straps (A Call of } \\
\text { the Wild reference). Embracing a challenge ([Employee } \\
\text { B]). (Written responses by Interviewees in response to } \\
\text { the following prompt: "Tell me about your star } \\
\text { employees. Tell me about the people who represent } \\
\text { the best of your organization." Written on the } \\
\text { Interview } 1 \text { Worksheet) }\end{array}$ & $\begin{array}{l}\text { Interview } 1 \\
\text { Worksheet }\end{array}$ \\
\hline & $\begin{array}{l}\text { Be willing to walk away from what you have spent } \\
\text { your life prepping for (college degree, training) if you } \\
\text { find that you're called to another field of endeavor. } \\
\text { (Written response by interviewee after the instruction } \\
\text { prompt to add any important language or behaviors } \\
\text { missing from the core values identification exercise) }\end{array}$ & $\begin{array}{l}\text { Value- } \\
\text { Behavior } \\
\text { Association } \\
\text { Worksheet, } \\
\text { [Employee D] }\end{array}$ \\
\hline & $\begin{array}{l}\text { Not placed in area of talent ([Employee C]). (In } \\
\text { response to the prompt: "Tell me about not-so-hot } \\
\text { employees. Tell me about the people who haven't or } \\
\text { wouldn't fit well with your organization") }\end{array}$ & $\begin{array}{l}\text { Interview with } \\
\text { [Employee C] }\end{array}$ \\
\hline
\end{tabular}


Table 8: Company B: Problem Solving DNA. Tracing Core Value "Problem Solving DNA" through constituent conceptual components and back to collected data at Company B. Underlined text is raw data, with context described parenthetically in italics.

\begin{tabular}{|c|c|c|}
\hline $\begin{array}{l}\text { Conceptual } \\
\text { Components: }\end{array}$ & Examples of Supporting Data: & Data Source: \\
\hline \multirow[t]{3}{*}{$\begin{array}{l}\text { Problem Solving } \\
\text { DNA: What we do is } \\
\text { solve problems for } \\
\text { customers. So we } \\
\text { hire and develop } \\
\text { people who have } \\
\text { the problem solving } \\
\text { DNA... } \\
\text { - Demonstrate the } \\
\text { DNA. Use your gifts } \\
\text { and abilities to find } \\
\text { and fix problems. }\end{array}$} & $\begin{array}{l}\text { Problem solver mentality ([Employee C]); Good logical } \\
\text { approach to problem solving. Always looking for } \\
\text { improvement opportunities ([Employee E]); Root cause } \\
\text { identification ([Employee A]); Synthesizer ([Employee } \\
\text { D]); Understand the entrepreneurial mind of the owner } \\
\text { ([Employee B]). (Written responses by Interviewees in } \\
\text { response to the following prompt: "Tell me about your } \\
\text { star employees. Tell me about the people who represent } \\
\text { the best of your organization." Written on the Interview } \\
1 \text { Worksheet) }\end{array}$ & $\begin{array}{l}\text { Interview } 1 \\
\text { Worksheet }\end{array}$ \\
\hline & $\begin{array}{l}\text { Gets hung up too easily. Needs step by step instruction. } \\
\text { Lacks problem solving ability. (In response to the prompt: } \\
\text { "Tell me about not-so-hot employees. Tell me about the } \\
\text { people who haven't or wouldn't fit well with your } \\
\text { organization") }\end{array}$ & $\begin{array}{l}\text { Interview with } \\
\text { [Employee E] }\end{array}$ \\
\hline & $\begin{array}{l}\text { Good at solving problems. (Written response by } \\
\text { interviewee after the instruction prompt to add any } \\
\text { important language or behaviors missing from the core } \\
\text { values identification exercise) }\end{array}$ & $\begin{array}{l}\text { Value-Behavior } \\
\text { Association } \\
\text { Worksheet, } \\
\text { [Employee B] }\end{array}$ \\
\hline \multirow{3}{*}{$\begin{array}{l}\text { We love new } \\
\text { challenges and } \\
\text { coming up with } \\
\text { unique solutions. } \\
\text { That's why we enjoy } \\
\text { taking on jobs that } \\
\text { others fail at or } \\
\text { avoid. }\end{array}$} & $\begin{array}{l}\text { Embracing a challenge. (In response to the prompt: "Tell } \\
\text { me about your star employees. Tell me about the people } \\
\text { who represent the best of your organization") }\end{array}$ & $\begin{array}{l}\text { Interview with } \\
\text { [Employee B] }\end{array}$ \\
\hline & $\begin{array}{l}\text { People who are afraid to fail or make mistakes (In } \\
\text { response to the prompt: "Tell me about not-so-hot } \\
\text { employees. Tell me about the people who haven't or } \\
\text { wouldn't fit well with your organization") }\end{array}$ & $\begin{array}{l}\text { Interview with } \\
\text { [Employee E] }\end{array}$ \\
\hline & $\begin{array}{l}\text { I suppose the other thing is recognize this is a small } \\
\text { company run by an entrepreneur. And it's not a big } \\
\text { corporation. So recognize the entrepreneurial spirit of } \\
\text { the owner. And that filters into everything. (In response } \\
\text { to the question: "Tell me, what is one piece of advice you } \\
\text { would give to a new employee or applicant about how to } \\
\text { thrive at this company?") }\end{array}$ & $\begin{array}{l}\text { Interview with } \\
\text { [Employee B] }\end{array}$ \\
\hline $\begin{array}{l}\text { We look for ways to } \\
\text { improve and } \\
\text { identify potential } \\
\text { problems before } \\
\text { they develop... } \\
\text { - Identify potential }\end{array}$ & $\begin{array}{l}\text { Detail oriented. Always looking for improvement } \\
\text { opportunities ([Employee E]); Initiator. Diligence } \\
\text { ([Employee B]). (Written responses by Interviewees in } \\
\text { response to the following prompt: "Tell me about your } \\
\text { star employees. Tell me about the people who represent } \\
\text { the best of your organization." Written on the Interview } \\
1 \text { Worksheet) }\end{array}$ & $\begin{array}{l}\text { Interview } 1 \\
\text { Worksheet }\end{array}$ \\
\hline
\end{tabular}




\begin{tabular}{|c|c|c|}
\hline $\begin{array}{l}\text { problems before } \\
\text { they show up. }\end{array}$ & $\begin{array}{l}\text { Identify problems before they show up. (Written } \\
\text { response by interviewee after the instruction prompt to } \\
\text { 'add any important language or behaviors missing from } \\
\text { the core values identification exercise') }\end{array}$ & $\begin{array}{l}\text { Value-Behavior } \\
\text { Association } \\
\text { Worksheet, } \\
\text { [Employee E] }\end{array}$ \\
\hline \multirow[t]{3}{*}{$\begin{array}{l}\text { Show a sense } \\
\text { of priority. Solve } \\
\text { the most important } \\
\text { problems first. }\end{array}$} & $\begin{array}{l}\text { Good logical approach to problem solving. (In response } \\
\text { to the prompt: "Tell me about your star employees. Tell } \\
\text { me about the people who represent the best of your } \\
\text { organization.") }\end{array}$ & $\begin{array}{l}\text { Interview with } \\
\text { [Employee E] }\end{array}$ \\
\hline & $\begin{array}{l}\text { Figure out the most important problem to solve FIRST. } \\
\text { (Written response by interviewee after the instruction } \\
\text { prompt to add any important language or behaviors } \\
\text { missing from the core values identification exercise) }\end{array}$ & $\begin{array}{l}\text { Value-Behavior } \\
\text { Association } \\
\text { Worksheet, } \\
\text { [Employee D] }\end{array}$ \\
\hline & $\begin{array}{l}\text { No sense of Priority ([Employee D]). (In response to the } \\
\text { prompt: "Tell me about not-so-hot employees. Tell me } \\
\text { about the people who haven't or wouldn't fit well with } \\
\text { your organization") }\end{array}$ & $\begin{array}{l}\text { Interview with } \\
\text { [Employee D] }\end{array}$ \\
\hline \multirow[t]{4}{*}{$\begin{array}{l}\text { Don't be } \\
\text { afraid to dig in, } \\
\text { learn, and ask } \\
\text { questions. }\end{array}$} & $\begin{array}{l}\text { Not afraid to dig in, learn, and ask questions (first } \\
\text { response)([Employee E]); Problem solver mentality } \\
\text { ([Employee C]); Be a learner. Own your progress. } \\
\text { ([Employee B]). (Written responses by Interviewees in } \\
\text { response to the following prompt: "Tell me about your } \\
\text { star employees. Tell me about the people who represent } \\
\text { the best of your organization." Written on the Interview } \\
1 \text { Worksheet) }\end{array}$ & $\begin{array}{l}\text { Interview } 1 \\
\text { Worksheet }\end{array}$ \\
\hline & $\begin{array}{l}\text { a big thing as a core value is to be a learner. Learn how } \\
\text { to learn your craft... learn how to assimilate information } \\
\text { that you're gonna need. Because to be a self-starter and } \\
\text { initiator will really really help your career here. That's a } \\
\text { highly valued trait. (In response to the question: "Tell me, } \\
\text { what is one piece of advice you would give to a new } \\
\text { employee or applicant about how to thrive at this } \\
\text { company?") }\end{array}$ & $\begin{array}{l}\text { Interview with } \\
\text { [Employee B] }\end{array}$ \\
\hline & $\begin{array}{l}\text { People who are afraid to fail or make mistakes (In } \\
\text { response to the prompt: "Tell me about not-so-hot } \\
\text { employees. Tell me about the people who haven't or } \\
\text { wouldn't fit well with your organization") }\end{array}$ & $\begin{array}{l}\text { Interview with } \\
\text { [Employee E] }\end{array}$ \\
\hline & $\begin{array}{l}\text { As l've watched over my } 8 \text { years here, different people } \\
\text { come and thrive, it's been the ones that have learned } \\
\text { how to learn. }\end{array}$ & $\begin{array}{l}\text { Interview with } \\
\text { [Employee B] }\end{array}$ \\
\hline $\begin{array}{l}\text {... the perseverance } \\
\text { to press forward } \\
\text { through challenges. } \\
\text { - Persevere. } \\
\text { Persistence is key. }\end{array}$ & $\begin{array}{l}\text { Stick to it until problem solved ([Employee E]); } \\
\text { Persistence ([Employee A]). (Written responses by } \\
\text { Interviewees in response to the following prompt: "Tell } \\
\text { me about your star employees. Tell me about the people } \\
\text { who represent the best of your organization." Written } \\
\text { on the Interview } 1 \text { Worksheet) }\end{array}$ & $\begin{array}{l}\text { Interview } 1 \\
\text { Worksheet }\end{array}$ \\
\hline
\end{tabular}


You're called upon to really really own the projects you work on, and you can't let up. You gotta stay at it, even though it's really hard.

Persistence. (Written response by interviewee after the instruction prompt to add any important language or behaviors missing from the core values identification exercise)
Interview with [Employee B]

Value-Behavior Association Worksheet, [Employee B] 
Table 9: Company B: A Culture of Integrity, Maturity \& Respectfulness. Tracing this Core Value through constituent conceptual components and back to data collected at Company B. Underlined text is raw data, with context described parenthetically in italics.

\begin{tabular}{|c|c|c|}
\hline $\begin{array}{l}\text { Conceptual } \\
\text { Components: }\end{array}$ & Examples of Supporting Data: & Data Source: \\
\hline \multirow[t]{3}{*}{$\begin{array}{l}\text { A Culture of } \\
\text { Integrity, Maturity } \\
\text { \& Respectfulness: } \\
\text { Having a healthy } \\
\text { culture allows us to } \\
\text { thrive. }\end{array}$} & $\begin{array}{l}\text { Humility. Character. Integrity. Willing to own } \\
\text { problems/mistakes ([Employee E]); Humble ([Employee } \\
\text { A]]); Selfless ([Employee D]); Humility ([Employee B]); We } \\
\text { value values ([Employee C]); Ownership attitude } \\
\text { ([Employee C]). (Written responses by Interviewees in } \\
\text { response to the following prompt: "Tell me about your } \\
\text { star employees. Tell me about the people who represent } \\
\text { the best of your organization." Written on the Interview } 1 \\
\text { Worksheet) }\end{array}$ & $\begin{array}{l}\text { Interview } 1 \\
\text { Worksheet }\end{array}$ \\
\hline & $\begin{array}{l}\text { Needs extra attention. (In response to the prompt: "Tell } \\
\text { me about not-so-hot employees. Tell me about the people } \\
\text { who haven't or wouldn't fit well with your organization") }\end{array}$ & $\begin{array}{l}\text { Interview with } \\
\text { [Employee E] }\end{array}$ \\
\hline & $\begin{array}{l}\text { So, the people who you look for and hire here, high talent, } \\
\text { high performance potential [and] making sure that there's } \\
\text { that humility piece so that they can work well with the } \\
\text { team? Yeah, and [they're] really hard to find. My first } 6 \\
\text { years here we just struggled to find that combination. } \\
\text { Somehow we've been blessed to find in the last year and } \\
\text { a half quite a number of young talented engineers that } \\
\text { have that native skill and ability, but aren't full of } \\
\text { themselves. }\end{array}$ & $\begin{array}{l}\text { Interview with } \\
\text { [Employee B] }\end{array}$ \\
\hline \multirow{3}{*}{$\begin{array}{l}\text { We treat our } \\
\text { customers, each } \\
\text { other, and our } \\
\text { community with } \\
\text { respect. Any } \\
\text { rudeness, } \\
\text { crudeness, } \\
\text { profanity, drama } \\
\text { or ego is out of } \\
\text { place here. }\end{array}$} & $\begin{array}{l}\text { Customer orientation \& service. ([Employee E]); We value } \\
\text { values ([Employee C]); Care about employees ([Employee } \\
\text { E] LT). (Written responses by Interviewees in response to } \\
\text { the following prompt: "Tell me about your star employees. } \\
\text { Tell me about the people who represent the best of your } \\
\text { organization." Written on the Interview } 1 \text { Worksheet) }\end{array}$ & $\begin{array}{l}\text { Interview } 1 \\
\text { Worksheet }\end{array}$ \\
\hline & $\begin{array}{l}\text { Good attitude at work. (Written response by interviewee } \\
\text { after the instruction prompt to add any important } \\
\text { language or behaviors missing from the core values } \\
\text { identification exercise) }\end{array}$ & $\begin{array}{l}\text { Value-Behavior } \\
\text { Association } \\
\text { Worksheet, } \\
\text { [Employee E] }\end{array}$ \\
\hline & $\begin{array}{l}\text { Respectful of co-workers/employees/vendors/customers. } \\
\text { (Written response by interviewee after the instruction } \\
\text { prompt to add any important language or behaviors } \\
\text { missing from the core values identification exercise) }\end{array}$ & $\begin{array}{l}\text { Value-Behavior } \\
\text { Association } \\
\text { Worksheet, } \\
\text { [Employee E] }\end{array}$ \\
\hline $\begin{array}{l}\text { We enjoy our } \\
\text { work, and enjoy } \\
\text { working together } \\
\text { as a team. }\end{array}$ & $\begin{array}{l}\text { Loves what they do, not here for the paycheck } \\
\text { ([Employee A]). (Written responses by Interviewees in } \\
\text { response to the following prompt: "Tell me about your } \\
\text { star employees. Tell me about the people who represent } \\
\text { the best of your organization." Written on the Interview } 1 \\
\text { Worksheet) }\end{array}$ & $\begin{array}{l}\text { Interview } 1 \\
\text { Worksheet }\end{array}$ \\
\hline
\end{tabular}




\begin{tabular}{|c|c|c|}
\hline & $\begin{array}{l}\text { Not team players. (In response to the prompt: "Tell me } \\
\text { about not-so-hot employees. Tell me about the people } \\
\text { who haven't or wouldn't fit well with your organization") }\end{array}$ & $\begin{array}{l}\text { Interview with } \\
\text { [Employee A] }\end{array}$ \\
\hline \multirow[t]{2}{*}{$\begin{array}{l}\text { Treat others } \\
\text { with dignity and } \\
\text { respect. }\end{array}$} & $\begin{array}{l}\text { Point out problems/errors with humility. (Written } \\
\text { response by interviewee after the instruction prompt to } \\
\text { add any important language or behaviors that are missing } \\
\text { from the core values identification exercise) }\end{array}$ & $\begin{array}{l}\text { Value-Behavior } \\
\text { Association } \\
\text { Worksheet, } \\
\text { [Employee E] }\end{array}$ \\
\hline & $\begin{array}{l}\text { Respectful of co-workers/employees/vendors/customers. } \\
\text { (Written response by interviewee after the instruction } \\
\text { prompt to add any important language or behaviors } \\
\text { missing from the core values identification exercise) }\end{array}$ & $\begin{array}{l}\text { Value-Behavior } \\
\text { Association } \\
\text { Worksheet, } \\
\text { [Employee E] }\end{array}$ \\
\hline \multirow[t]{2}{*}{$\begin{array}{l}\text { Always do } \\
\text { the right thing. No } \\
\text { short cuts. }\end{array}$} & $\begin{array}{l}\text { Doesn't take shortcuts that affect someone else. (Written } \\
\text { response by interviewee after the instruction prompt to } \\
\text { add any important language or behaviors missing from } \\
\text { the core values identification exercise) }\end{array}$ & $\begin{array}{l}\text { Value-Behavior } \\
\text { Association } \\
\text { Worksheet, } \\
\text { [Employee E] }\end{array}$ \\
\hline & $\begin{array}{l}\text { Integrity. Stick to it until problem solved. Willing to own } \\
\text { problems/mistakes ([Employee E]). (Written responses by } \\
\text { Interviewees in response to the following prompt: "Tell me } \\
\text { about your star employees. Tell me about the people who } \\
\text { represent the best of your organization." Written on the } \\
\text { Interview } 1 \text { Worksheet) }\end{array}$ & $\begin{array}{l}\text { Interview } 1 \\
\text { Worksheet }\end{array}$ \\
\hline \multirow{3}{*}{$\begin{array}{l}\text { We believe } \\
\text { everything we do } \\
\text { should be done } \\
\text { well. } \bullet \text { Work hard. } \\
\text { There is immense } \\
\text { dignity in a task } \\
\text { well done. }\end{array}$} & $\begin{array}{l}\text { "No task is too small a vessel to hold the immense dignity } \\
\text { of work..." (A Tim Keller quote written as a response by } \\
\text { interviewee after the instruction prompt to add any } \\
\text { important language or behaviors missing from the core } \\
\text { values identification exercise) }\end{array}$ & $\begin{array}{l}\text { Value-Behavior } \\
\text { Association } \\
\text { Worksheet, } \\
\text { [Employee D] }\end{array}$ \\
\hline & $\begin{array}{l}\text { Hard worker. (Written response by interviewee after the } \\
\text { instruction prompt to add any important language or } \\
\text { behaviors missing from the core values identification } \\
\text { exercise) }\end{array}$ & $\begin{array}{l}\text { Value-Behavior } \\
\text { Association } \\
\text { Worksheet, } \\
\text { [Employee B] }\end{array}$ \\
\hline & $\begin{array}{l}\text { Detail oriented. Always looking for improvement } \\
\text { opportunities ([Employee E]); Diligence ([Emplovee B]]; } \\
\text { embracing a challenge ([Employee B]). (Written responses } \\
\text { by Interviewees in response to the following prompt: "Tell } \\
\text { me about your star employees. Tell me about the people } \\
\text { who represent the best of your organization." Written on } \\
\text { the Interview } 1 \text { Worksheet) }\end{array}$ & $\begin{array}{l}\text { Interview } 1 \\
\text { Worksheet }\end{array}$ \\
\hline \multirow[t]{2}{*}{$\begin{array}{l}\text { - Do } \\
\text { whatever is } \\
\text { needed, even if it is } \\
\text { inconvenient. }\end{array}$} & $\begin{array}{l}\text { You're called upon to really really own the projects you } \\
\text { work on, and you can't let up. You gotta stay at it, even } \\
\text { though it's really hard. And somehow you have to learn } \\
\text { to enjoy being in the trenches that are hard like that, and } \\
\text { that's not an easy path for most people I don't think. }\end{array}$ & $\begin{array}{l}\text { Interview with } \\
\text { [Employee B] }\end{array}$ \\
\hline & $\begin{array}{l}\text { Do whatever is needed. (Written response by interviewee } \\
\text { after the instruction prompt to add any important } \\
\text { language or behaviors missing from the core values } \\
\text { identification exercise) }\end{array}$ & $\begin{array}{l}\text { Value-Behavior } \\
\text { Association } \\
\text { Worksheet, } \\
\text { [Employee E] }\end{array}$ \\
\hline
\end{tabular}




\begin{tabular}{|c|c|c|}
\hline & $\begin{array}{l}\text { Integrity ([Employee E]); Working with integrity } \\
\text { ([Employee D] LT); Ownership attitude ([Employee C]). } \\
\text { (Written responses by Interviewees in response to the } \\
\text { following prompt: "Tell me about your star employees. } \\
\text { Tell me about the people who represent the best of your } \\
\text { organization." Written on the Interview } 1 \text { Worksheet) }\end{array}$ & $\begin{array}{l}\text { Interview } 1 \\
\text { Worksheet }\end{array}$ \\
\hline \multirow[t]{7}{*}{$\begin{array}{l}\text { - Be a team } \\
\text { player. None of us } \\
\text { are perfect. But } \\
\text { together we } \\
\text { surpass our } \\
\text { individual limits. }\end{array}$} & $\begin{array}{l}\text { None of us are perfect - we need to extend/receive ... } \\
\text { to/from others. (Written response by interviewee after } \\
\text { the instruction prompt to add any important language or } \\
\text { behaviors missing from the core values identification } \\
\text { exercise) }\end{array}$ & $\begin{array}{l}\text { Value-Behavior } \\
\text { Association } \\
\text { Worksheet, } \\
\text { [Employee D] }\end{array}$ \\
\hline & $\begin{array}{l}\text { Humility ([Employee E]); Humble ([Employee A]); Selfless } \\
\text { ([Employee D]); Humility ([Employee B]). (Written } \\
\text { responses by Interviewees in response to the following } \\
\text { prompt: "Tell me about your star employees. Tell me } \\
\text { about the people who represent the best of your } \\
\text { organization." Written on the Interview } 1 \text { Worksheet) }\end{array}$ & $\begin{array}{l}\text { Interview } 1 \\
\text { Worksheet }\end{array}$ \\
\hline & $\begin{array}{l}\text { if you're ego-driven, it's going to be really really hard to } \\
\text { thrive. And that has effect on your co-workers, huge } \\
\text { effect on how you come across with customers, and both } \\
\text { of those are really a big deal for the overall success of the } \\
\text { company. }\end{array}$ & $\begin{array}{l}\text { Interview with } \\
\text { [Employee B] }\end{array}$ \\
\hline & $\begin{array}{l}\text { Not team players. (In response to the prompt: "Tell me } \\
\text { about not-so-hot employees. Tell me about the people } \\
\text { who haven't or wouldn't fit well with your organization") }\end{array}$ & $\begin{array}{l}\text { Interview with } \\
\text { [Employee A] }\end{array}$ \\
\hline & $\begin{array}{l}\text { Builds others up. (Written response by interviewee after } \\
\text { the instruction prompt to add any important language or } \\
\text { behaviors missing from the core values identification } \\
\text { exercise) }\end{array}$ & $\begin{array}{l}\text { Value-Behavior } \\
\text { Association } \\
\text { Worksheet, } \\
\text { [Employee E] }\end{array}$ \\
\hline & $\begin{array}{l}\text { Cleans up after self \& others. (Written response by } \\
\text { interviewee after the instruction prompt to add any } \\
\text { important language or behaviors missing from the core } \\
\text { values identification exercise) }\end{array}$ & $\begin{array}{l}\text { Value-Behavior } \\
\text { Association } \\
\text { Worksheet, } \\
\text { [Employee E] }\end{array}$ \\
\hline & $\begin{array}{l}\text { Looks to help others who are struggling. (Written } \\
\text { response by interviewee after the instruction prompt to } \\
\text { add any important language or behaviors missing from } \\
\text { the core values identification exercise) }\end{array}$ & $\begin{array}{l}\text { Value-Behavior } \\
\text { Association } \\
\text { Worksheet, } \\
\text { [Employee E] }\end{array}$ \\
\hline
\end{tabular}


Table 10: Company C: The Company C Winning Mindset. Tracing Core Value "The [Company C] Winning Mindset" through constituent conceptual components and back to collected data at Company C during the initial data collection phase. Underlined text is raw data, with context described parenthetically in italics.

\begin{tabular}{|c|c|c|}
\hline $\begin{array}{l}\text { Conceptual } \\
\text { Components: }\end{array}$ & Examples of Supporting Data: & Data Source: \\
\hline \multicolumn{3}{|c|}{ Theme: Winning. } \\
\hline \multirow[t]{5}{*}{ Winning. } & $\begin{array}{l}\text { star employees... let's see... just people who have a history of } \\
\text { winning. (In response to the prompt: "Tell me about your star } \\
\text { employees. Tell me about the people who represent the best } \\
\text { of your organization") }\end{array}$ & $\begin{array}{l}\text { Interview with } \\
\text { [Employee B] }\end{array}$ \\
\hline & $\begin{array}{l}\text { So we have some people that played college sports and } \\
\text { competed at a high level, and I think that just helps because } \\
\text { you get used to winning. (In response to the prompt: "Tell me } \\
\text { about your star employees. Tell me about the people who } \\
\text { represent the best of your organization") }\end{array}$ & $\begin{array}{l}\text { Interview with } \\
\text { [Employee B] }\end{array}$ \\
\hline & $\begin{array}{l}\text { And, in an online space, there's very clear winners and losers. } \\
\text { And so, being able to get your clients to a confident space } \\
\text { where we can say, yes, you are now winning, is very helpful. } \\
\text { (In response to the prompt: "Tell me about your star } \\
\text { employees. Tell me about the people who represent the best } \\
\text { of your organization") }\end{array}$ & $\begin{array}{l}\text { Interview with } \\
\text { [Employee B] }\end{array}$ \\
\hline & $\begin{array}{l}\text { Talking about employees: because we're not locked in with } \\
\text { contracts, they have to have that desire to really do what's } \\
\text { right, do what's best. }\end{array}$ & $\begin{array}{l}\text { Interview with } \\
\text { [Employee C] }\end{array}$ \\
\hline & $\begin{array}{l}\text { Winning ([Employee B]); Team "win" or team mentality } \\
\text { ([Employee E]); Positive competition (competing with as } \\
\text { opposed to against) ([Employee E]); Winners ([Employee A]). } \\
\text { (Written responses by Interviewees in response to the } \\
\text { following prompt: "Tell me about your star employees. Tell } \\
\text { me about the people who represent the best of your } \\
\text { organization." Written on the Interview } 1 \text { Worksheet) }\end{array}$ & $\begin{array}{l}\text { Interview } 1 \\
\text { Worksheet }\end{array}$ \\
\hline \multirow[t]{2}{*}{$\begin{array}{l}\text { We succeed } \\
\text { through ... great } \\
\text { strategy }\end{array}$} & $\begin{array}{l}\text { Strategy is huge.... they typically have the strategy mind } \\
\text { that's required to look at this data and figure out how to get } \\
\text { this client to win in their industry. (In response to the } \\
\text { prompt: "Tell me about your star employees. Tell me about } \\
\text { the people who represent the best of your organization") }\end{array}$ & $\begin{array}{l}\text { Interview with } \\
\text { [Employee B] }\end{array}$ \\
\hline & $\begin{array}{l}\text { Strategy ([Employee B]); Gamers ([Employee B]); Highly } \\
\text { intelligent ([Employee B] LT); Critical Thinkers ([Employee E]); } \\
\text { Derives success from getting better results ([Employee F]); } \\
\text { High vision ([Employee A] LT). (Written responses by } \\
\text { Interviewees in response to the following prompt: "Tell me } \\
\text { about your star employees. Tell me about the people who } \\
\text { represent the best of your organization." Written on the } \\
\text { Interview } 1 \text { Worksheet) }\end{array}$ & $\begin{array}{l}\text { Interview } 1 \\
\text { Worksheet }\end{array}$ \\
\hline
\end{tabular}




\begin{tabular}{|c|c|c|}
\hline \multirow[t]{5}{*}{$\begin{array}{l}\text { We succeed } \\
\text { through ... } \\
\text { general } \\
\text { awesomeness }\end{array}$} & $\begin{array}{l}\text { Strategy is huge. It's almost - it's become actually part of my } \\
\text { interview questioning, if they're a gamer, which surprises me } \\
\text { because I never thought l'd be asking people if they're a } \\
\text { gamer. Gaming for me was like, playing James Bond in } \\
\text { college... but strategy.. if they're a successful gamer, they } \\
\text { typically have the strategy mind that's required to look at } \\
\text { this data and figure out how to get this client to win in their } \\
\text { industry...Yeah. So those real-time strategy games, that's } \\
\text { what we do in essence, with other people's money. So it's } \\
\text { weird. Strategies, gamers, yeah. (In response to the prompt: } \\
\text { "Tell me about your star employees. Tell me about the } \\
\text { people who represent the best of your organization") }\end{array}$ & $\begin{array}{l}\text { Interview with } \\
\text { [Employee B] }\end{array}$ \\
\hline & $\begin{array}{l}\text { There is sign hanging next to the calendar in the break room } \\
\text { that says "Not all superheroes wear capes." (This } \\
\text { communicates, reinforces, and is a symbol of the playful, } \\
\text { young, game-y, quirky group of people there, and a } \\
\text { celebration of who they are in their work space) }\end{array}$ & Observation \\
\hline & $\begin{array}{l}\text { There is another large sign that hangs over the toaster in the } \\
\text { break room and says "Keep Calm and DFTBA." (DFTBA means } \\
\text { 'don't forget to be awesome'). }\end{array}$ & Observation \\
\hline & $\begin{array}{l}\text { Well, you've been here on Fridays: we give out tickets. It's a } \\
\text { way to, you know, give kudos to people... when we recognize } \\
\text { each of us in our team doing something awesome: 'hey I } \\
\text { want to give you a ticket to recognize what you did'... we } \\
\text { want to recognize, 'hey man, you did a great job, you deserve } \\
\text { some acknowledgement for that'. That's a huge thing that } \\
\text { was a big thing for me, coming to the company ... from other } \\
\text { jobs where it was all about, you know... I wouldn't say } \\
\text { individual achievement but, there was no acknowledgement } \\
\text { of success in things like that. }\end{array}$ & $\begin{array}{l}\text { Key Informant } \\
\text { Leader Interview } \\
\text { [Employee E] }\end{array}$ \\
\hline & $\begin{array}{l}\text { My first trip to the break room, I found two senior account } \\
\text { managers heating up lunch and joking with each other in the } \\
\text { room. They both engaged me quickly and naturally, asking } \\
\text { 'how's it going', and introducing themselves and each other. } \\
\text { One described the other as "awesome at life in general." }\end{array}$ & Observation \\
\hline
\end{tabular}




\begin{tabular}{|c|c|c|}
\hline & $\begin{array}{l}\text { The first ticket given was a big one; it was given to an } \\
\text { employee for being up on a specific client } 80 \% \text { year over } \\
\text { year. Every year getting } 80 \% \text { more spend than the year } \\
\text { before, showing that the client trusted her a tremendous } \\
\text { amount because her performance was so good... Another } \\
\text { employee was given for his great work on for a specific } \\
\text { account, and he was told by teammates that "you are a } \\
\text { shining star." People laughed a little, others smiled - all in } \\
\text { jovialness... Next, "Ticket to Employee Y for helping me with } \\
\text { my reports." "Ticket to Employee Z for being awesome and } \\
\text { diligent." "Ticket to H for wrangling cats." People laughed in } \\
\text { response to this one. (These field notes reflect Participant } \\
\text { Observation at the Friday morning all-hands meeting, where } \\
\text { tickets are given by/to employees for performance, which are } \\
\text { redeemed at the end of quarters for rewards). }\end{array}$ & Observation \\
\hline \multirow[t]{7}{*}{$\begin{array}{l}\text { - Driven. } \\
\text { Passionate. } \\
\text { Strategic. } \\
\text { Always seeking } \\
\text { better ways to } \\
\text { do the best } \\
\text { possible job. }\end{array}$} & $\begin{array}{l}\text { You know, the biggest thing that I would say that we at least } \\
\text { encourage people is that it is really up to them to take } \\
\text { control of their own growth here ... it's just up to the } \\
\text { individual employee if they're going to have that drive and } \\
\text { that motivation to do it. (In response to the question: "Tell } \\
\text { me, what is one piece of advice you would give to a new } \\
\text { employee or applicant about how to thrive at this } \\
\text { company?") }\end{array}$ & $\begin{array}{l}\text { Interview with } \\
\text { [Employee C] }\end{array}$ \\
\hline & $\begin{array}{l}\text { the people who have been successful ... have a desire to } \\
\text { learn how to help their client in the best way possible. And } \\
\text { so, if they focus on finding the best way to service the client } \\
\text { as possible then they're going to be successful. That's the } \\
\text { attitude that it takes. }\end{array}$ & $\begin{array}{l}\text { Interview with } \\
\text { [Employee D] }\end{array}$ \\
\hline & $\begin{array}{l}\text { We're very supportive, and we'll help them along the way, } \\
\text { but ultimately they have to have that drive and desire on } \\
\text { their own. (referring to employees) }\end{array}$ & $\begin{array}{l}\text { Interview with } \\
\text { [Employee C] }\end{array}$ \\
\hline & $\begin{array}{l}\text { there's a lot of opportunity here, but it's completely up to } \\
\text { you with how fast you will advance and grow in our } \\
\text { company. So, and to do that, you really have to be motivated } \\
\text { to learn. }\end{array}$ & $\begin{array}{l}\text { Interview with } \\
\text { [Employee D] }\end{array}$ \\
\hline & $\begin{array}{l}\text { because we hire people who don't have experience, so we } \\
\text { try to hire off of passion and motivation, and someone who } \\
\text { wants to learn. }\end{array}$ & $\begin{array}{l}\text { Interview with } \\
\text { [Employee D] }\end{array}$ \\
\hline & $\begin{array}{l}\text { You've gotta have that drive inside yourself to learn and do } \\
\text { better. And that's what makes, I think, really successful } \\
\text { people in the company is that drive. }\end{array}$ & $\begin{array}{l}\text { Interview with } \\
\text { [Employee D] }\end{array}$ \\
\hline & $\begin{array}{l}\text { you know, firing our sales team I think was huge, because } \\
\text { that forced us to focus on the client and produce the best } \\
\text { product possible. And so producing the best product possible } \\
\text { is all the sales force we need. }\end{array}$ & $\begin{array}{l}\text { Interview with } \\
\text { [Employee D] }\end{array}$ \\
\hline
\end{tabular}




\begin{tabular}{|c|c|c|}
\hline & $\begin{array}{l}\text { I think we're all pretty driven. We are, some more than } \\
\text { others, competitive. We want to do better than our } \\
\text { competition. We want to do better than other companies } \\
\text { that are advertising for businesses and things like that. So } \\
\text { there is a driving force in addition to that accountability [to } \\
\text { clients] that we have. }\end{array}$ & $\begin{array}{l}\text { Key Informant } \\
\text { Leader Interview, } \\
\text { [Employee E] }\end{array}$ \\
\hline & $\begin{array}{l}\text {... when they instituted the paid time off and the flexible } \\
\text { hours, nobody took advantage of it for the first couple } \\
\text { months, and they actually had to force us to take time off. } \\
\text { And, you know, I think that speaks to the level of 'people' } \\
\text { and the desire to do well. }\end{array}$ & $\begin{array}{l}\text { Key Informant } \\
\text { Leader Interview, } \\
{[\text { Employee E] }}\end{array}$ \\
\hline & $\begin{array}{l}\text { Motivated. Self-motivated. Passionate about what we do. } \\
\text { Passionate for clients. Problem Solving and Troubleshooting } \\
\text { ([Employee D]); People who are motivated ([Employee C]]; } \\
\text { Passion. Desire to go above and beyond. Loves learning. } \\
\text { Always seeks better results. Derives success from getting } \\
\text { better results ([Employee F]); Driven - results oriented, } \\
\text { positive competition. Passionate for the job. Have \& } \\
\text { encourage personal goals ([Employee E]); Strategy. Winning. } \\
\text { Highly intelligent ([Employee B]). (Written responses by } \\
\text { Interviewees in response to the following prompt: "Tell me } \\
\text { about your star employees. Tell me about the people who } \\
\text { represent the best of your organization." Written on the } \\
\text { Interview } 1 \text { Worksheet) }\end{array}$ & $\begin{array}{l}\text { Interview } 1 \\
\text { Worksheet }\end{array}$ \\
\hline \multirow[t]{3}{*}{$\begin{array}{l}\text { - Your } \\
\text { "standard } \\
\text { overachiever." } \\
\text { Holds } \\
\text { themselves to } \\
\text { extremely high } \\
\text { personal } \\
\text { standards. }\end{array}$} & $\begin{array}{l}\text { so typically where we've found the most success, I generally } \\
\text { look for kind of your standard over-achiever. So you know, } \\
\text { the people that in school, weren't satisfied with the B, they } \\
\text { wanted straight A's and A +'s, just because they had } \\
\text { something to prove kind of for themselves. They just held } \\
\text { themselves to a higher standard, and wanted to always be } \\
\text { sure they were always producing the best (In response to the } \\
\text { prompt: "Tell me about your star employees. Tell me about } \\
\text { the people who represent the best of your organization") }\end{array}$ & $\begin{array}{l}\text { Interview with } \\
\text { [Employee C]1 } \\
\text { Star }\end{array}$ \\
\hline & $\begin{array}{l}\text { people who take pride in their work, and the result. (In } \\
\text { response to the prompt: "Tell me about your star employees. } \\
\text { Tell me about the people who represent the best of your } \\
\text { organization") }\end{array}$ & $\begin{array}{l}\text { Interview with } \\
\text { [Employee D] }\end{array}$ \\
\hline & $\begin{array}{l}\text { we look for over-achievers, people who are self-motivated, } \\
\text { we definitely look for intelligent people. (In response to the } \\
\text { prompt: "Tell me about your star employees. Tell me about } \\
\text { the people who represent the best of your organization") }\end{array}$ & $\begin{array}{l}\text { Interview with } \\
\text { [Employee C] }\end{array}$ \\
\hline
\end{tabular}




\begin{tabular}{|c|c|c|}
\hline & $\begin{array}{l}\text { Your standard overachiever. Hold themselves to a higher } \\
\text { standard ([Employee C]); Desire to go above and beyond. } \\
\text { Always seeking better results. Derives success from getting } \\
\text { better results ([Employee F]); Driven - results oriented } \\
\text { ([Employee E]); Strong work ethic. Self-motivated ([Employee } \\
\text { D]). (Written responses by Interviewees in response to the } \\
\text { following prompt: "Tell me about your star employees. Tell } \\
\text { me about the people who represent the best of your } \\
\text { organization." Written on the Interview } 1 \text { Worksheet) }\end{array}$ & $\begin{array}{l}\text { Interview } 1 \\
\text { Worksheet }\end{array}$ \\
\hline & $\begin{array}{l}\text { They're motivated. Very, very motivated. (In response to the } \\
\text { prompt: "Tell me about your star employees. Tell me about } \\
\text { the people who represent the best of your organization") }\end{array}$ & $\begin{array}{l}\text { Interview with } \\
\text { [Employee D] Star }\end{array}$ \\
\hline & Theme: Learning. & \\
\hline $\begin{array}{l}\text { - Relentless } \\
\text { Personal } \\
\text { Development. A } \\
\text { desire to learn }\end{array}$ & $\begin{array}{l}\text { some of the training that l've done has helped me realize } \\
\text { what they were doing to me the two years before -- which } \\
\text { was a very relentless effort to develop me personally and } \\
\text { relationally. (referring to the leadership team) }\end{array}$ & $\begin{array}{l}\text { Interview with } \\
\text { [Employee D] }\end{array}$ \\
\hline $\begin{array}{l}\text { skills, because } \\
\text { our industry } \\
\text { changes so } \\
\text { quickly. }\end{array}$ & $\begin{array}{l}\text { In response to the question: "Tell me, what is one piece of } \\
\text { advice you would give to a new employee or applicant about } \\
\text { how to thrive at this company?": ...you have got to be able } \\
\text { to, to a degree develop yourself. Like we can get you to a } \\
\text { point, but your success in this industry depends on you } \\
\text { having a desire to learn and develop new skills. And if you } \\
\text { can't - because our industry changes so quickly. And what } \\
\text { worked } 6 \text { months ago doesn't work now. And so if you're } \\
\text { not in that constant, you know, reading articles and getting } \\
\text { things are going, you're going to get left behind because } \\
\text { things are changing so quickly. }\end{array}$ & $\begin{array}{l}\text { Interview with } \\
\text { [Employee B]1 Q1 }\end{array}$ \\
\hline & $\begin{array}{l}\text { things are changing so quickly. So personal development } \\
\text { should be a core value of your own. (In response to the } \\
\text { question: "Tell me, what is one piece of advice you would give } \\
\text { to a new employee or applicant about how to thrive at this } \\
\text { company?") }\end{array}$ & $\begin{array}{l}\text { Interview with } \\
\text { [Employee B] }\end{array}$ \\
\hline & $\begin{array}{l}\text { [Company C] started in a completely different industry. The } \\
\text { CEO demonstrated that learning new skills is in the leadership } \\
\text { DNA with his response to this question: "Ok, so the } \\
\text { knowledge about this industry space, where does that come } \\
\text { from?" We had to learn it. We didn't know anything about it } \\
\text { when we started. }\end{array}$ & $\begin{array}{l}\text { Interview with } \\
\text { [Employee B] }\end{array}$ \\
\hline
\end{tabular}




\begin{tabular}{|c|c|c|}
\hline & $\begin{array}{l}\text { You know, the biggest thing that I would say that we at least } \\
\text { encourage people is that it is really up to them to take } \\
\text { control of their own growth here. We give a lot of } \\
\text { opportunities to people to really grow and develop... it's just } \\
\text { up to the individual employee if they're going to have that } \\
\text { drive and that motivation to do it. (In response to the } \\
\text { question: "Tell me, what is one piece of advice you would give } \\
\text { to a new employee or applicant about how to thrive at this } \\
\text { company?") }\end{array}$ & $\begin{array}{l}\text { Interview with } \\
\text { [Employee C] }\end{array}$ \\
\hline & $\begin{array}{l}\text { So if you're the type of person who doesn't like change, and } \\
\text { can't handle this, I usually say that this probably isn't going to } \\
\text { be the best fit for you, because it's going to happen. (In } \\
\text { response to the question: "Tell me, what is one piece of } \\
\text { advice you would give to a new employee or applicant about } \\
\text { how to thrive at this company?") }\end{array}$ & $\begin{array}{l}\text { Interview with } \\
\text { [Employee C] }\end{array}$ \\
\hline & $\begin{array}{l}\text { we try to hire off of passion and motivation, and someone } \\
\text { who wants to learn. Those are the people who have been } \\
\text { successful that are self-motivated to learn. They're reading } \\
\text { outside of work, they, you know, they have a desire to learn } \\
\text { what we do. }\end{array}$ & $\begin{array}{l}\text { Interview with } \\
\text { [Employee D] }\end{array}$ \\
\hline & $\begin{array}{l}\text { I think it's really just that everything's changing, so you just } \\
\text { really have to be learning and keeping up on stuff all the time } \\
\text { and constantly developing yourself and letting us develop } \\
\text { you }\end{array}$ & $\begin{array}{l}\text { Interview with } \\
\text { [Employee D] }\end{array}$ \\
\hline & $\begin{array}{l}\text { You've gotta have that drive inside yourself to learn and do } \\
\text { better. And that's what makes, I think, really successful } \\
\text { people in the company is that drive. }\end{array}$ & $\begin{array}{l}\text { Interview with } \\
\text { [Employee D] }\end{array}$ \\
\hline & $\begin{array}{l}\text { part of our development that the management and } \\
\text { leadership focus on is personal development... it's big, I } \\
\text { mean it goes hand in hand with your professional } \\
\text { development. So learning things like time management and } \\
\text { how to raise your EQ versus your IQ }\end{array}$ & $\begin{array}{l}\text { Interview with } \\
\text { [Employee D] }\end{array}$ \\
\hline & Theme: Leading. & \\
\hline Leadership. All & $\begin{array}{l}\ldots . . \text { I mean, since we hire everybody new with no experience, } \\
\text { you have to be able to move up. }\end{array}$ & $\begin{array}{l}\text { Interview with } \\
\text { [Employee B] }\end{array}$ \\
\hline $\begin{array}{l}\text { employees are in } \\
\text { leadership roles } \\
\text { or have }\end{array}$ & $\begin{array}{l}\text { We want all of our star employees to be leaders. That's } \\
\text { probably the biggest thing. (In response to the probe, "Is } \\
\text { there anything else [about star employees]?") }\end{array}$ & $\begin{array}{l}\text { Interview with } \\
\text { [Employee C] }\end{array}$ \\
\hline
\end{tabular}




\begin{tabular}{|l|l|}
\hline $\begin{array}{l}\text { leadership } \\
\text { potential. }\end{array}$ & $\begin{array}{l}\text { There was a 'trial employee' present on Wednesday. He was } \\
\text { a PSU business school graduate, and was positioned at a desk } \\
\text { near the north end of the room. He took interest in my study } \\
\text { and asked me some questions. Management had let him } \\
\text { know about my study during his interview. He shared with } \\
\text { me that during the interview process, he believed that the } \\
\text { things they were looking for the most were ... potential or } \\
\text { ability to be a leader, a... (Field note excerpt from participant } \\
\text { observation) }\end{array}$
\end{tabular}

Participant

Observation

Theme: Together / Team Mentality.

\begin{tabular}{|c|c|c|}
\hline \multirow{2}{*}{$\begin{array}{l}\text { Together. We } \\
\text { succeed through } \\
\text { teamwork... }\end{array}$} & ...it's a very collaborative, teamwork kind of environment. & $\begin{array}{l}\text { Interview with } \\
\text { [Employee C] }\end{array}$ \\
\hline & $\begin{array}{l}\text {...everything we do was based off of my strategies to begin } \\
\text { with. Now, those are far beyond what I ever could have } \\
\text { imagined... Oh, it's amazing. Especially when you can free } \\
\text { yourself from competition and pride. Which has been } \\
\text { another hard thing for me is being humble and not caring } \\
\text { about receiving the credit for things, because who cares at } \\
\text { the end of the day as long as it gets done. Your needs are } \\
\text { being met and so are the client's. (reflecting on how much } \\
\text { some of his ideas were improved through iterative and } \\
\text { collaborative group processes) }\end{array}$ & $\begin{array}{l}\text { Interview with } \\
\text { [Employee D] }\end{array}$ \\
\hline \multirow{3}{*}{$\begin{array}{l}\quad \text { Team } \\
\text { Mentality. } \\
\text { We're all in this } \\
\text { together. } \\
\text { Building others } \\
\text { up \& caring } \\
\text { about the } \\
\text { success of } \\
\text { coworkers. }\end{array}$} & $\begin{array}{l}\text { And we really rely on our team members to help train other } \\
\text { team members. And so, because that's the model that we've } \\
\text { built, we have to have people who care about the success } \\
\text { about their co-workers, and can rely in them and trust in } \\
\text { them. }\end{array}$ & $\begin{array}{l}\text { Interview with } \\
\text { [Employee C] }\end{array}$ \\
\hline & $\begin{array}{l}\text { Probe: And then, you talked about competing together rather } \\
\text { than competing against each other. Yeah. You know, I just } \\
\text { didn't really realize how much I was competing against 'no } \\
\text { one' I guess. Like how, I think it was a lot of past job } \\
\text { experiences... it's just a different perspective. Much more } \\
\text { team atmosphere than competition. We're all in it together. } \\
\text { And that makes a big difference. }\end{array}$ & $\begin{array}{l}\text { Interview with } \\
\text { [Employee D] }\end{array}$ \\
\hline & $\begin{array}{l}\text { Team "win" or team mentality ([Employee E]); Develop Team } \\
\text { ([Employee B] LT); Training others/caring about the success } \\
\text { of their co-workers ([Employee C]); Want to help others. } \\
\text { Wants [company] to succeed ([Employee F]); Invested in the } \\
\text { success of the company as a whole ([Employee D]). (Written } \\
\text { responses by Interviewees in response to the following } \\
\text { prompt: "Tell me about your star employees. Tell me about } \\
\text { the people who represent the best of your organization." } \\
\text { Written on the Interview } 1 \text { Worksheet) }\end{array}$ & $\begin{array}{l}\text { Interview } 1 \\
\text { Worksheet }\end{array}$ \\
\hline
\end{tabular}


Table 11: Company C: Caring for others. Tracing Core Value "Caring for others" through constituent conceptual components and back to collected data at Company $\mathrm{C}$ during the initial data collection phase. Underlined text is raw data, with context described parenthetically in italics.

\begin{tabular}{|c|c|c|}
\hline $\begin{array}{l}\text { Conceptual } \\
\text { Components: }\end{array}$ & Examples of Supporting Data: & Data Source: \\
\hline \multicolumn{3}{|c|}{ Theme: Caring } \\
\hline \multirow[t]{7}{*}{$\begin{array}{l}\text { Caring for } \\
\text { others. Caring is } \\
\text { at our core. }\end{array}$} & $\begin{array}{l}\text { (describing the company culture) .... place that cares most } \\
\text { about the employees first and foremost . So our employees } \\
\text { come first, before the clients. We kind of figure that as long } \\
\text { as we're taking care of our employees, the employees can } \\
\text { take care of the clients, the clients take care of the } \\
\text { business, and it's all this happy circle that everyone gets } \\
\text { taken care of, as long as that's kind of our focus. }\end{array}$ & $\begin{array}{l}\text { Interview with } \\
\text { [Employee C] }\end{array}$ \\
\hline & $\begin{array}{l}\text { CEO, talking about employees: So your job is to take care of } \\
\text { the client. And we structure our organization, we're } \\
\text { serving the senior account managers, to the best of your } \\
\text { ability, helping them look good to their clients that they're } \\
\text { taking care of. }\end{array}$ & $\begin{array}{l}\text { Interview with } \\
\text { [Employee B] }\end{array}$ \\
\hline & $\begin{array}{l}\text { You know, the people who succeed here are people who } \\
\text { really care about people. }\end{array}$ & $\begin{array}{l}\text { Interview with } \\
\text { [Employee C] }\end{array}$ \\
\hline & $\begin{array}{l}\text { Compassionate ([Employee E] LT); People focused } \\
\text { ([Employee E] LT); Authentic Care - personally \& } \\
\text { professionally ([Employee E] LT); Service, helping others } \\
\text { ([Employee B]). (Written responses by Interviewees in } \\
\text { response to the following prompt: "Tell me about your star } \\
\text { employees. Tell me about the people who represent the } \\
\text { best of your organization." Written on the Interview } 1 \\
\text { Worksheet) }\end{array}$ & $\begin{array}{l}\text { Interview } 1 \\
\text { Worksheet }\end{array}$ \\
\hline & It's all about the people here and just focusing on them. & $\begin{array}{l}\text { Interview with } \\
\text { [Employee C] }\end{array}$ \\
\hline & $\begin{array}{l}\text { But really our main focus is just to take care of our people } \\
\text { and bless them. }\end{array}$ & $\begin{array}{l}\text { Interview with } \\
\text { [Employee C] }\end{array}$ \\
\hline & $\begin{array}{l}\text { An employee recounts interviewing with [Company C] } \\
\text { before being hired: And so I went out and talked to them, } \\
\text { felt their heart for the client, their attitude of service for } \\
\text { their client, and where their focuses were, and so that's } \\
\text { when I decided to come and work for them for half of what } \\
\text { Istated was my pay when we started negotiations. And } \\
\text { regardless came and worked here because I believe in what } \\
\text { they're doing. }\end{array}$ & $\begin{array}{l}\text { Interview with } \\
\text { [Employee D] }\end{array}$ \\
\hline $\begin{array}{l}\text { - Caring for } \\
\text { each other }\end{array}$ & $\begin{array}{l}\text { So when you come to this organization, understand that } \\
\text { we're going to take care of you. You're not going to have } \\
\text { to worry about that. }\end{array}$ & $\begin{array}{l}\text { Interview with } \\
\text { [Employee B] }\end{array}$ \\
\hline
\end{tabular}




\begin{tabular}{|c|c|c|}
\hline \multirow[t]{4}{*}{$\begin{array}{l}\text { personally; for } \\
\text { employees and } \\
\text { their families. }\end{array}$} & $\begin{array}{l}\text { We want our people to have come here and be made } \\
\text { better in some way, shape, or form. Whether it be that } \\
\text { they grew professionally, or personally, or we were able to } \\
\text { help their family, or whatever it may be. }\end{array}$ & $\begin{array}{l}\text { Interview with } \\
\text { [Employee C] }\end{array}$ \\
\hline & $\begin{array}{l}\text { I think a lot of it is all of the 'behind the scenes' of what we } \\
\text { do to support employees on a personal level. (In response } \\
\text { to the question, 'What would you say is the best part about } \\
\text { working here at [Company C] that someone like me might } \\
\text { not be able to figure out by spending a week here observing } \\
\text { or asking good questions - what's something that I might } \\
\text { miss?') }\end{array}$ & $\begin{array}{l}\text { Interview with } \\
\text { [Employee C] }\end{array}$ \\
\hline & $\begin{array}{l}\text { [Employee B] recounts a story of generosity and a desire for } \\
\text { the personal success of employees, where the leadership } \\
\text { team voluntarily decides to offer a savings program and } \\
\text { financial rewards for employees improving their position in } \\
\text { life: ....at the beginning of the year we decided we're going } \\
\text { to do a } 6 \text { months saving program... we said, for } 6 \text { months } \\
\text { you're going to sit with [Employee C], weekly, monthly, you } \\
\text { or you and your spouse if you want to bring them... and } \\
\text { we'll go through and map all of your finances, set some } \\
\text { goals... and for that } 6 \text { month period ... all the money that } \\
\text { you save or pay off in debt, we'll match, up to } \$ 5,000 . \\
\text { Because we know that you're going to make more money } \\
\text { at [Company C], we want to make sure you're actually } \\
\text { improving your position in life. }\end{array}$ & $\begin{array}{l}\text { Interview with } \\
\text { (a shorter } \\
\text { version exists } \\
\text { down in the } \\
\text { generosity } \\
\text { section) } \\
\text { [Employee B] }\end{array}$ \\
\hline & $\begin{array}{l}\text { authentic care - personally \& professionally ([Employee E] } \\
\text { LT); Emphasis on personal development ([Employee F] LT); } \\
\text { Want to help others ([Employee F]); Development of } \\
\text { people personally ([Employee D] LT); Speaking life over } \\
\text { employees and clients ([Employee D] LT). (Written } \\
\text { responses by Interviewees in response to the following } \\
\text { prompt: "Tell me about your star employees. Tell me about } \\
\text { the people who represent the best of your organization." } \\
\text { Written on the Interview } 1 \text { Worksheet) }\end{array}$ & $\begin{array}{l}\text { Interview } 1 \\
\text { Worksheet }\end{array}$ \\
\hline \multicolumn{3}{|c|}{ Theme: Passion } \\
\hline & The people we hire, we hire for passion. & $\begin{array}{l}\text { Interview with } \\
\text { [Employee A] }\end{array}$ \\
\hline \multirow{2}{*}{$\begin{array}{l}\text { Passionate } \\
\text { about serving } \\
\text { the clients } \\
\text { extremely well. }\end{array}$} & $\begin{array}{l}\text { This organization exists because we serve our clients } \\
\text { extremely well. }\end{array}$ & $\begin{array}{l}\text { Interview with } \\
\text { (with CEO) }\end{array}$ \\
\hline & $\begin{array}{l}\text { And as an agency, we're pretty strange that way because, I } \\
\text { don't really put a lot of focus on making sure the } \\
\text { organization, [Company C], gets their name out or people } \\
\text { know who we are or care who we are. Our focus has been } \\
\text { specifically with making sure our clients win. }\end{array}$ & $\begin{array}{l}\text { Interview with } \\
\text { [Employee B] }\end{array}$ \\
\hline
\end{tabular}




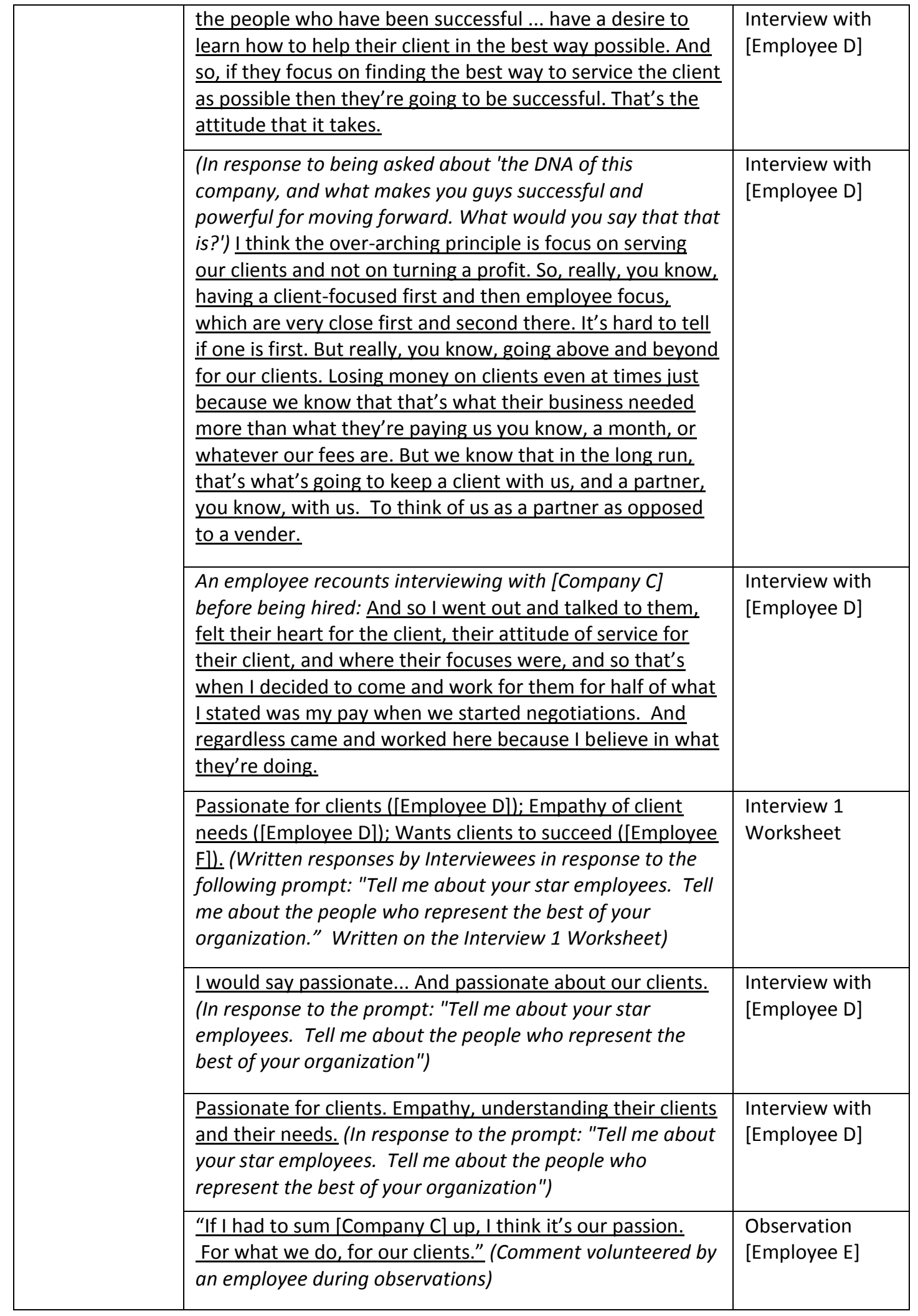




\begin{tabular}{|c|c|c|}
\hline \multirow[t]{4}{*}{$\begin{array}{l}\text { Passionate } \\
\text { about what we } \\
\text { do. Always } \\
\text { seeking better } \\
\text { results. }\end{array}$} & $\begin{array}{l}\text { I would say passionate... passionate about marketing. They } \\
\text { enjoy it. They enjoy what they do. Passionate about what } \\
\text { we do. (In response to the prompt: "Tell me about your star } \\
\text { employees. Tell me about the people who represent the } \\
\text { best of your organization") }\end{array}$ & $\begin{array}{l}\text { Interview with } \\
\text { [Employee D] } \\
\text { Star }\end{array}$ \\
\hline & $\begin{array}{l}\text { we've always been performance-based. We've never had } \\
\text { the long-term contracts. }\end{array}$ & $\begin{array}{l}\text { Interview with } \\
\text { [Employee C] }\end{array}$ \\
\hline & $\begin{array}{l}\text { Passionate for the job ([Employee E]); Passion. Desire to go } \\
\text { above and beyond ([Employee F]); Always seeking better } \\
\text { results ([Employee F]); Derives success from getting better } \\
\text { results ([Employee F]); Passionate about what we do } \\
\text { ([Employee D]); Take pride in their work and results } \\
\text { ([Employee D]); Looking for a career instead of a job } \\
\text { ([Employee D]); Willing to sacrifice ([Employee A]). (Written } \\
\text { responses by Interviewees in response to the following } \\
\text { prompt: "Tell me about your star employees. Tell me about } \\
\text { the people who represent the best of your organization." } \\
\text { Written on the Interview } 1 \text { Worksheet) }\end{array}$ & $\begin{array}{l}\text { Interview } 1 \\
\text { Worksheet }\end{array}$ \\
\hline & $\begin{array}{l}\text {...you know, firing our sales team I think was huge, because } \\
\text { that forced us to focus on the client and produce the best } \\
\text { product possible. And so producing the best product } \\
\text { possible is all the sales force we need. }\end{array}$ & $\begin{array}{l}\text { Interview with } \\
\text { [Employee D] }\end{array}$ \\
\hline \multirow[t]{4}{*}{$\begin{array}{l}\text { Helping / } \\
\text { Serving }\end{array}$} & $\begin{array}{l}\text { Understand how to serve. ([Employee B]'s first words in } \\
\text { response to the prompt: "Tell me about your star } \\
\text { employees. Tell me about the people who represent the } \\
\text { best of your organization") }\end{array}$ & $\begin{array}{l}\text { Interview with } \\
\text { [Employee B] }\end{array}$ \\
\hline & $\begin{array}{l}\text { Service Mentality. Whether for other employees or the } \\
\text { client ([Employee A]); Want to help others ([Employee F]); } \\
\text { Compassionate ([Employee E] LT); Service, helping others } \\
\text { ([Employee B]). (Written responses by Interviewees in } \\
\text { response to the following prompt: "Tell me about your star } \\
\text { employees. Tell me about the people who represent the } \\
\text { best of your organization." Written on the Interview } 1 \\
\text { Worksheet) }\end{array}$ & $\begin{array}{l}\text { Interview } 1 \\
\text { Worksheet }\end{array}$ \\
\hline & $\begin{array}{l}\text { A core belief is that extremely good service creates success: } \\
\text { And I came to believe that if you're producing a } \\
\text { phenomenal product, and your clients... ...if they're making } \\
\text { a great return on their investment online, they're gonna } \\
\text { want to invest more. }\end{array}$ & $\begin{array}{l}\text { Interview with } \\
\text { [Employee B] }\end{array}$ \\
\hline & $\$ 100$ a week we give away to encourage service behavior & $\begin{array}{l}\text { Interview with } \\
\text { [Employee B] }\end{array}$ \\
\hline
\end{tabular}




\begin{tabular}{|c|c|c|}
\hline \multirow[t]{4}{*}{$\begin{array}{l}\text { We exist } \\
\text { because we } \\
\text { serve our clients } \\
\text { extremely well. }\end{array}$} & $\begin{array}{l}\text { Well if you enter this company to put yourself first, or try to } \\
\text { put the company first, you're not going to succeed. This } \\
\text { organization exists because we serve our clients extremely } \\
\text { well. (In response to the question: "Tell me, what is one } \\
\text { piece of advice you would give to a new employee or } \\
\text { applicant about how to thrive at this company?") }\end{array}$ & $\begin{array}{l}\text { Interview with } \\
\text { (with CEO) } \\
\text { [Employee B] }\end{array}$ \\
\hline & $\begin{array}{l}\text { The leadership have really been trying to instill these values } \\
\text { in me... One of those being a servant's heart and service to } \\
\text { your clients. And stewarding what you've been given well. }\end{array}$ & $\begin{array}{l}\text { Interview with } \\
\text { [Employee D] }\end{array}$ \\
\hline & $\begin{array}{l}\text { we } 100 \% \text { of the time put the clients' needs above ours and } \\
\text { say, if it makes sense for the client we need to do it, and } \\
\text { we'll figure out the back end how we make it work - but } \\
\text { our first thing is not "it has to work for [Company C] first" - } \\
\text { that doesn't come into play or cause situations or } \\
\text { problems. (In response to the question: "Tell me, what is } \\
\text { one piece of advice you would give to a new employee or } \\
\text { applicant about how to thrive at this company?") }\end{array}$ & $\begin{array}{l}\text { Interview with } \\
\text { [Employee B] }\end{array}$ \\
\hline & $\begin{array}{l}\text { Passion, Desire to go above and beyond ([Employee F]); } \\
\text { Ownership of accounts ([Employee F]); Growth of Clients } \\
\text { ([Employee F] LT); We're performance based, we don't lock } \\
\text { our clients into contracts ([Employee C]); take pride in their } \\
\text { work and results ([Employee D]); Willingness to sacrifice } \\
\text { ([Employee A]). (Written responses by Interviewees in } \\
\text { response to the following prompt: "Tell me about your star } \\
\text { employees. Tell me about the people who represent the } \\
\text { best of your organization." Written on the Interview } 1 \\
\text { Worksheet) }\end{array}$ & $\begin{array}{l}\text { Interview } 1 \\
\text { Worksheet }\end{array}$ \\
\hline \multirow{2}{*}{$\begin{array}{l}\text { We understand } \\
\text { that serving } \\
\text { others also } \\
\text { fulfills the } \\
\text { servant, so we } \\
\text { focus on others } \\
\text { rather than } \\
\text { ourselves. }\end{array}$} & $\begin{array}{l}\text {.... as an agency, we're pretty strange that way because, } \\
\text { don't really put a lot of focus on making sure the } \\
\text { organization, [Company Cl, gets their name out or people } \\
\text { know who we are or care who we are. Our focus has been } \\
\text { specifically with making sure our clients win. }\end{array}$ & $\begin{array}{l}\text { Interview with } \\
\text { [Employee B] }\end{array}$ \\
\hline & $\begin{array}{l}\text { (Describing how the leadership team actively seeks to } \\
\text { develop employees, and the response from employees) } \\
\text { [The leadership team pursues]... just development as } \\
\text { people, because they know that that creates good } \\
\text { employees as well. Plus once you realize what they're } \\
\text { pouring into you, it makes you want to pour it back into } \\
\text { them. }\end{array}$ & $\begin{array}{l}\text { Interview, } \\
\text { [Employee D] }\end{array}$ \\
\hline $\begin{array}{l}\text { Passionate } \\
\text { about serving } \\
\text { each other and }\end{array}$ & $\begin{array}{l}\text { Learn to be a servant. (In response to the question: "Tell } \\
\text { me, what is one piece of advice you would give to a new } \\
\text { employee or applicant about how to thrive at this } \\
\text { company?") }\end{array}$ & $\begin{array}{l}\text { Leader Interview } \\
\text { [Employee B] }\end{array}$ \\
\hline
\end{tabular}




\begin{tabular}{|c|c|c|}
\hline \multirow[t]{5}{*}{$\begin{array}{l}\text { helping each } \\
\text { other succeed } \\
\text { professionally. } \\
\text { Developing } \\
\text { others. }\end{array}$} & $\begin{array}{l}\text {...some of the training that I've done has helped me realize } \\
\text { what they were doing to me the two years before -- which } \\
\text { was a very relentless effort to develop me personally and } \\
\text { relationally. }\end{array}$ & $\begin{array}{l}\text { Interview with } \\
\text { [Employee D] }\end{array}$ \\
\hline & $\begin{array}{l}\text { the leadership team... really does focus on developing the } \\
\text { rest of the team and raising them up. }\end{array}$ & $\begin{array}{l}\text { Interview with } \\
\text { [Employee B] }\end{array}$ \\
\hline & $\begin{array}{l}\text { It kind of goes back to the core of why I feel we are } \\
\text { successful. It's that the leadership has decided to invest in } \\
\text { people and not necessarily in the things that are going to } \\
\text { help the company grow. They feel like the things that are } \\
\text { going to help the company grow is by investing in people, } \\
\text { not investing in things that are business needs that people } \\
\text { can use. But we're gonna build up the best people; we're } \\
\text { gonna create the best people, we're gonna train the best } \\
\text { people; we're just gonna find the best people and have the } \\
\text { best people working for us. That's the mentality that I } \\
\text { think has made us so successful }\end{array}$ & $\begin{array}{l}\text { Key Informant } \\
\text { Interview with } \\
\text { [Employee E] }\end{array}$ \\
\hline & $\begin{array}{l}\text { Talking about how employees must act with integrity and } \\
\text { caring toward clients and co-workers: because we're not } \\
\text { locked in with contracts, they have to have that desire to } \\
\text { really do what's right, do what's best. And not only for the } \\
\text { client, but also for the employees, as co-workers. }\end{array}$ & $\begin{array}{l}\text { Interview with } \\
\text { [Employee C] }\end{array}$ \\
\hline & $\begin{array}{l}\text { I view it as my job to make sure that everyone out there } \\
\text { has everything they need to do their job. Because I know if } \\
\text { they have that, they're going to do what they need to do } \\
\text { what they need to do to take care of the client, which, } \\
\text { ultimately, pays the bills at the end of the day. You know? }\end{array}$ & $\begin{array}{l}\text { Interview with } \\
\text { [Employee C] }\end{array}$ \\
\hline \multicolumn{3}{|c|}{ Theme: Generosity } \\
\hline \multirow[t]{2}{*}{$\begin{array}{l}\text { Generosity } \\
\text { fulfills the giver, } \\
\text { and we get that. }\end{array}$} & $\begin{array}{l}\text { So by helping the people we are serving, the whole } \\
\text { organization is winning, which is great. But by helping } \\
\text { those that we are serving directly in the marketing } \\
\text { department in those organizations, help them look good to } \\
\text { the people who are looking at them, it's basically helping } \\
\text { us. So we're serving them to a degree, and by default, it's } \\
\text { going to help us in the long term but our focus isn't on that } \\
\text { because we know it's taken care of. }\end{array}$ & $\begin{array}{l}\text { Interview with } \\
\text { [Employee B] }\end{array}$ \\
\hline & $\begin{array}{l}\text { Oh, that's easy (he says immediately), generosity. (In } \\
\text { response to the question, 'What would you say is the best } \\
\text { part about working here at [Company C] that someone like } \\
\text { me might not be able to figure out by spending a week here } \\
\text { observing or asking good questions - what's something } \\
\text { that I might miss?') }\end{array}$ & $\begin{array}{l}\text { Interview with } \\
\text { [Employee B] }\end{array}$ \\
\hline
\end{tabular}




\begin{tabular}{|c|c|c|}
\hline & $\begin{array}{l}\text { So, as an organization, [the owner] and I [the CEO] have } \\
\text { made it a point, along with [the operations manager], that } \\
\text { we are going to be generous to our employees. (In response } \\
\text { to the question, 'What would you say is the best part about } \\
\text { working here at [Company C] that someone like me might } \\
\text { not be able to figure out by spending a week here observing } \\
\text { or asking good questions - what's something that I might } \\
\text { miss?') }\end{array}$ & $\begin{array}{l}\text { Interview with } \\
\text { [Employee B] }\end{array}$ \\
\hline & $\begin{array}{l}\text { [Employee B] recounts a story of transparency and } \\
\text { generosity that began when the company was a struggling } \\
\text { startup: We snapped a budget, we had the margin, we } \\
\text { were still trying to figure out how to manage this, so we } \\
\text { knew we weren't going to be able to pay people enough to } \\
\text { justify their time. And we told them that, and they knew it. } \\
\text { "We're underpaying; we know this. Our goal is to get you } \\
\text { to livable wage quickly, and move beyond that. We want to } \\
\underline{\text { be generous with things and we can't be." So we've }} \\
\text { continued that, even as their wages have caught up and } \\
\text { gone probably beyond what a lot of them would make } \\
\text { somewhere else. }\end{array}$ & $\begin{array}{l}\text { Interview with } \\
\text { [Employee B] }\end{array}$ \\
\hline & $\begin{array}{l}\text { [Company C] offers a voluntary 6-month savings program } \\
\text { for employees, and the CEO recounts that: all the money } \\
\text { that you save or pay off in debt, we're gonna match, up to } \\
\$ 5,000 \text {. Because we know that you're going to make more } \\
\text { money at [Company Cl, we want to make sure you're } \\
\text { actually improving your position in life. }\end{array}$ & $\begin{array}{l}\text { Interview with } \\
\text { [Employee B]1 }\end{array}$ \\
\hline & $\begin{array}{l}\text { CEO describes one piece of [Company C]s caring about the } \\
\text { long-term personal sucess of employees: we want to make } \\
\text { sure you're... preparing for retirement, so we actually help } \\
\text { pay for a financial advisor to the } 401 \mathrm{k} \text {. We do a matching } \\
401 \mathrm{k} \text { which, I don't know if any other agencies in Portland } \\
\text { do that, because we want them investing in their } \\
\text { retirement. }\end{array}$ & $\begin{array}{l}\text { Interview with } \\
\text { [Employee B] }\end{array}$ \\
\hline & $\begin{array}{l}\text { as an organization we value generosity and we donate } \\
\text { some time to children's cancer association, managing their } \\
\text { stuff, their paid search, and we donate time to them, some } \\
\text { of their projects around the city of Portland. I said, we } \\
\text { really want to impact people, and we want to do it in a way } \\
\text { we can actually see the impact too. }\end{array}$ & $\begin{array}{l}\text { Interview with } \\
\text { [Employee B] }\end{array}$ \\
\hline
\end{tabular}




\begin{tabular}{|l|l|l|}
\hline $\begin{array}{l}\text { (In response to being asked about 'the DNA of this } \\
\text { company, and what makes you guys successful and } \\
\text { powerful for moving forward. What would you say that that } \\
\text { is?') I think the over-arching principle is focus on serving } \\
\text { our clients and not on turning a profit. So, really, you know, }\end{array}$ & $\begin{array}{l}\text { Interview with } \\
\text { [Employee D] } \\
\text { having a client-focused first and then employee focus, } \\
\begin{array}{l}\text { which are very close first and second there. It's hard to tell } \\
\text { if one is first. But really, you know, going above and beyond } \\
\text { for our clients. Losing money on clients even at times just }\end{array}\end{array}$ & $\begin{array}{l}\text { because we know that that's what their business needed } \\
\text { more than what they're paying us you know, a month, or }\end{array}$ \\
$\begin{array}{l}\text { whatever our fees are. But we know that in the long run, } \\
\text { that's what's going to keep a client with us, and a partner, } \\
\text { you know, with us. To think of us as a partner as opposed } \\
\text { to a vender. }\end{array}$ & \\
\hline
\end{tabular}


Table 12: Company C: Balanced Analytical \& Emotional Intelligence. Tracing the Core Value "Balanced Analytical \& Emotional Intelligence" through constituent conceptual components and back to data collected during the initial data collection phase at Company C. Underlined text is raw data, with context described parenthetically in italics.

\begin{tabular}{|l|l|l|}
\hline $\begin{array}{l}\text { Conceptual } \\
\text { Components: }\end{array}$ & Examples of Supporting Data: & Data Source: \\
\hline
\end{tabular}

Theme: Technical Prowess

\begin{tabular}{|c|c|}
\hline $\begin{array}{l}\text { - Analytical } \\
\text { intelligence. Able } \\
\text { to sift through a lot } \\
\text { of data and } \\
\text { produce insight. }\end{array}$ & $\begin{array}{l}\text { Analytical / Math ([Employee B]]; Analytical ([Employee A]); } \\
\text { Analytical ([Employee E]); Highly Intelligent ([Employee B]]); } \\
\text { Intelligent people. Able to sift through a lot of numbers and } \\
\text { produce insight ([Employee C]); Critical thinkers ([Employee E]) } \\
\text { (Written responses by Interviewees in response to the following } \\
\text { prompt: "Tell me about your star employees. Tell me about the } \\
\text { people who represent the best of your organization." Written } \\
\text { on the Interview } 1 \text { Worksheet) }\end{array}$ \\
\hline
\end{tabular}

...have a very analytical, mathematical mind. (In response to the prompt: "Tell me about your star employees. Tell me about the people who represent the best of your organization")

We just look at data so much, you have to be able to look at data, and see patterns and make decisions (In response to the prompt: "Tell me about your star employees. Tell me about the people who represent the best of your organization")

...we definitely look for intelligent people. You know, we work a lot with numbers, and so people who are able to sift through large amounts of data and gain insight from that is really helpful. (In response to the prompt: "Tell me about your star employees. Tell me about the people who represent the best of your organization")

...when it comes to marketing, there's definitely two different sides, there is a creative side, which is very important, but that's just not what we do. We're very data and numbers centric, and so I can usually tell if they're more into creatives and that type of thing, that this is just going to drive them crazy. (In response to the prompt: "Tell me about not-so-hot employees. Tell me about the people who haven't or wouldn't fit well with your organization")

Google Analytics and other spreadsheets are often up on at least one screen, and data is viewed regarding view rates, click thoughs, etc. (Memo: Data Analysis is a core job function)
Interview 1

Worksheet

Interview with [Employee B]

Interview with [Employee B]

Interview with [Employee C]

Interview with [Employee C]

Observation [Employee E] 


\begin{tabular}{|c|c|c|}
\hline \multirow[t]{2}{*}{$\begin{array}{l}\text { - Problem Solving } \\
\text { and } \\
\text { Troubleshooting } \\
\text { Skills. }\end{array}$} & $\begin{array}{l}\text { Problem solving skills, just good at trouble shooting and } \\
\text { problem solving. That's so much of what we do. And so just } \\
\text { knowing how to start ruling things out. And then start, yeah, a } \\
\text { lot of what we're doing is just digging through things, finding } \\
\text { problems, find the outliers, and that kind of stuff. (In response } \\
\text { to the prompt: "Tell me about your star employees. Tell me } \\
\text { about the people who represent the best of your organization") }\end{array}$ & $\begin{array}{l}\text { Interview with } \\
\text { [Employee D] }\end{array}$ \\
\hline & $\begin{array}{l}\text { Problem Solving and troubleshooting ([Employee D]); Always } \\
\text { seeking better results ([Employee F]); Derives success from } \\
\text { getting better results ([Employee F]); Analytical ([Employee B], } \\
{[\text { [Employee A], [Employee E]); (Critical thinkers ([Employee E]); }} \\
\text { Winning ([Employee B]); Winners ([Employee A]). (Written } \\
\text { responses by Interviewees in response to the following prompt: } \\
\text { "Tell me about your star employees. Tell me about the people } \\
\text { who represent the best of your organization." Written on the } \\
\text { Interview } 1 \text { Worksheet) }\end{array}$ & $\begin{array}{l}\text { Interview } 1 \\
\text { Worksheet }\end{array}$ \\
\hline \multirow[t]{4}{*}{$\begin{array}{l}\text { Readiness to } \\
\text { learn and re-learn } \\
\text { every day as our } \\
\text { industry changes } \\
\text { constantly. }\end{array}$} & $\begin{array}{l}\text { I mean it's not necessarily something they can do, per se, I } \\
\text { would look at it more as just an overall kind of quality about } \\
\text { them that they have, but they need to be able to handle } \\
\text { change. We, a) the industry that we're in changes a lot and b) } \\
\text { the fact that we're a small company, we still are kind of digging } \\
\text { through the trenches and trying to make our path, and so } \\
\text { things change quite a bit around here. (In response to the } \\
\text { question: "Tell me, what is one piece of advice you would give } \\
\text { to a new employee or applicant about how to thrive at this } \\
\text { company?") }\end{array}$ & $\begin{array}{l}\text { Interview with } \\
\text { [Employee C] }\end{array}$ \\
\hline & $\begin{array}{l}\text { the flexibility that we have, like the agile-ness that we have as a } \\
\text { company is very important because I think a lot of agencies } \\
\text { become stuck in ways and are reluctant to change. And in our } \\
\text { industry we become very outdated very quickly. And so I think } \\
\text { our acceptance of change and that things are always going to } \\
\text { be changing, we just need to change them for something } \\
\text { better, just the acceptance-ness and the flexibility of the } \\
\text { company I think is huge to us growing and figuring out what the } \\
\text { best way to do this is. }\end{array}$ & $\begin{array}{l}\text { Interview with } \\
\text { [Employee D] }\end{array}$ \\
\hline & $\begin{array}{l}\text { Again people who aren't able to adapt to change, whether it's } \\
\text { internal change, or change in the industry and that type of } \\
\text { thing, have not been all that successful }\end{array}$ & $\begin{array}{l}\text { Interview with } \\
\text { [Employee C] }\end{array}$ \\
\hline & $\begin{array}{l}\text { Probe: I like that, that kind of the flexibility and agility that is so } \\
\text { much at the core there, just kind of flows from that. Yeah, and I } \\
\text { think it gives us the ability to serve our clients better. }\end{array}$ & $\begin{array}{l}\text { Interview with } \\
\text { [Employee D] }\end{array}$ \\
\hline
\end{tabular}




\begin{tabular}{|c|c|}
\hline $\begin{array}{l}\text { I would say 'think outside the box'. And be flexible as a reed. } \\
\text { Because the nature of our job, the nature of what we do } \\
\text { changes constantly. New things are being rolled out, } \\
\text { specifically with Google because that's where the majority of } \\
\text { our work is with Google adwords; and adwords changes } \\
\text { constantly. So being able to think outside the box, being } \\
\text { creative, is almost a necessity in this type of work. And as far } \\
\text { as being flexible like a reed also plays into the changes. } \\
\text { Because everything changes within our business, google } \\
\text { adwords changes, also people change. Trends change. So we } \\
\text { have to be flexible enough to change with them. We have to } \\
\text { predict those changes that come into play. (In response to the } \\
\text { question: "Tell me, what is one piece of advice you would give } \\
\text { to a new employee or applicant about how to thrive at this } \\
\text { company?") }\end{array}$ & $\begin{array}{l}\text { Key Informant } \\
\text { Interview with } \\
\text { [Employee E] }\end{array}$ \\
\hline $\begin{array}{l}\text { In response to this (summarizing) probe: "So always be } \\
\text { listening, and learning, and relearning?" Yep. I like that. That's } \\
\text { actually better. Always be learning, because there's always } \\
\text { new things that are coming up so, the more you know, the } \\
\text { more you learn, the better you're going to be at this job. }\end{array}$ & $\begin{array}{l}\text { KI Interview } \\
\text { with } \\
\text { [Employee E] }\end{array}$ \\
\hline $\begin{array}{l}\text { Loves learning ([Employee F]); Tries new things ([Employee F]); } \\
\text { Eagerness to learn ([Employee D]). (Written responses by } \\
\text { Interviewees in response to the following prompt: "Tell me } \\
\text { about your star employees. Tell me about the people who } \\
\text { represent the best of your organization." Written on the } \\
\text { Interview } 1 \text { Worksheet) }\end{array}$ & $\begin{array}{l}\text { Interview } 1 \\
\text { Worksheet }\end{array}$ \\
\hline
\end{tabular}

Theme: People Skills

\begin{tabular}{|c|c|c|}
\hline & $\begin{array}{l}\text {...some of the training that I've done has helped me realize } \\
\text { what they were doing to me the two years before -- which was } \\
\text { a very relentless effort to develop me personally and } \\
\text { relationally. }\end{array}$ & $\begin{array}{l}\text { Interview with } \\
\text { [Employee D] }\end{array}$ \\
\hline & $\begin{array}{l}\text {...you know, IQ has very low correlation with professional } \\
\text { success... it is really more about your EQ and being able to } \\
\text { relate to people and that kind of stuff that makes you } \\
\text { successful professionally. Not only professionally but } \\
\text { personally as well, as being married and having a wife has } \\
\text { taught me. }\end{array}$ & $\begin{array}{l}\text { Interview with } \\
\text { [Employee D] }\end{array}$ \\
\hline $\begin{array}{l}\text { - Easy to talk to. } \\
\text { Approachable. } \\
\text { Positive and } \\
\text { Respectful. }\end{array}$ & $\begin{array}{l}\text { If they're not very personable, you know, again, we } \\
\text { communicate with each other a lot, and communicate with our } \\
\text { clients a lot, and if they give kind of a weird vibe (gentle laugh), } \\
\text { and if they're just not easy to talk to, that's a pretty good } \\
\text { indicator that they're not going to fit in very well here. (In } \\
\text { response to the prompt: "Tell me about not-so-hot employees. } \\
\text { Tell me about the people who haven't or wouldn't fit well with } \\
\text { your organization") }\end{array}$ & $\begin{array}{l}\text { Interview with } \\
\text { [Employee C] }\end{array}$ \\
\hline
\end{tabular}




\begin{tabular}{|c|c|c|}
\hline & $\begin{array}{l}\text { Leading by example and just, just always trying to you know, } \\
\text { have a positive outlook, our clients, and treating our clients in a } \\
\text { positive way. (describing key behaviors) }\end{array}$ & $\begin{array}{l}\text { Interview with } \\
\text { [Employee D] }\end{array}$ \\
\hline & $\begin{array}{l}\text {...for the personality and belonging here, I would say, be } \\
\text { positive. You know? We don't like complainers, whiners; we } \\
\text { always want to be positive in how we view our clients, and also } \\
\text { each other. There's definitely a level of respect with each } \\
\text { other that we always maintain. (In response to a follow-up } \\
\text { probe on the question: "Tell me, what is one piece of advice you } \\
\text { would give to a new employee or applicant about how to thrive } \\
\text { at this company?") }\end{array}$ & $\begin{array}{l}\text { Key Informant } \\
\text { Interview with } \\
\text { [Employee E] }\end{array}$ \\
\hline & $\begin{array}{l}\text { Emotional intelligence ([Emplovee A]); Communication } \\
\text { ([Employee B]); Vocal (shares thoughts, insights, etc) } \\
\text { ([Employee E]); Sociable, approachable, \& personable } \\
\text { ([Employee E]); Personable ([Employee F]) (Written responses } \\
\text { by Interviewees in response to the following prompt: "Tell me } \\
\text { about your star employees. Tell me about the people who } \\
\text { represent the best of your organization." Written on the } \\
\text { Interview } 1 \text { Worksheet) }\end{array}$ & $\begin{array}{l}\text { Interview } 1 \\
\text { Worksheet }\end{array}$ \\
\hline \multirow{5}{*}{$\begin{array}{l}\text { - Have faith in } \\
\text { people. Default to } \\
\text { noticing the } \\
\text { goodness or } \\
\text { potential in others. }\end{array}$} & $\begin{array}{l}\text { And most of the leadership team does default to believing the } \\
\text { best about people. }\end{array}$ & $\begin{array}{l}\text { Interview with } \\
\text { [Employee B] }\end{array}$ \\
\hline & $\begin{array}{l}\text { Describing his thoughts on 'having faith in people', [Employee } \\
\text { B] says: I think that those things can be learned.. it takes a very } \\
\text { long time though to have your default be the best view of } \\
\text { somebody first, rather than believe the worst and then try to } \\
\text { get them to live up to something. }\end{array}$ & $\begin{array}{l}\text { Interview with } \\
\text { [Employee B] }\end{array}$ \\
\hline & $\begin{array}{l}\text {...to have a strong belief in people: like giving the benefit of the } \\
\text { doubt. }\end{array}$ & $\begin{array}{l}\text { Interview with } \\
\text { [Employee C] }\end{array}$ \\
\hline & $\begin{array}{l}\text { (Responding to a prompt about the qualities of leaders at the } \\
\text { company): ...they have to have a strong belief in people: like } \\
\text { giving the benefit of the doubt. So, you know, when we are } \\
\text { looking at leaders... or looking at bringing people up in the } \\
\text { company and putting them more in a type of a leadership role, } \\
\text { that's probably one of the biggest things that they have to be } \\
\text { able to do. }\end{array}$ & $\begin{array}{l}\text { Interview with } \\
\text { [Employee C] }\end{array}$ \\
\hline & $\begin{array}{l}\text { (Responding to a prompt about the qualities of leaders at the } \\
\text { company): ...they have to be able to look at the rest of the } \\
\text { employees in this company with positivity and always assume } \\
\text { that whatever the employee is doing or is not doing is coming } \\
\text { with the best intentions, it's coming from the best place, that } \\
\text { they mean well by it, that they have the ability to do it. }\end{array}$ & $\begin{array}{l}\text { Interview with } \\
\text { [Employee C] }\end{array}$ \\
\hline
\end{tabular}




\begin{tabular}{|c|c|c|}
\hline & $\begin{array}{l}\text { Have a strong faith in people ([Employee C]); Giving people the } \\
\text { benefit of the doubt ([Employee C]); Have to look at employees } \\
\text { with positivity ([Employee C]); Default - Best of others } \\
\text { ([Employee B]). (Written responses by Interviewees in response } \\
\text { to the following prompt: "Tell me about your Leadership Team." } \\
\text { In almost all cases, the values and behaviors of the leadership } \\
\text { were equated with those of star employees. Written on the } \\
\text { Interview } 1 \text { Worksheet) }\end{array}$ & $\begin{array}{l}\text { Interview } 1 \\
\text { Worksheet }\end{array}$ \\
\hline \multirow[t]{3}{*}{$\begin{array}{l}\text { - Authenticity, } \\
\text { Integrity, Owning } \\
\text { up to mistakes, } \\
\text { Being real. }\end{array}$} & $\begin{array}{l}\text { We need people who have a lot of integrity. We're } \\
\text { performance based. We don't lock our clients into contracts. So } \\
\text { we need to be able to show every single month that we're } \\
\text { providing the value that the client is paying for. So we really } \\
\text { need employees who are going to do a stellar job. And, you } \\
\text { know, we're very transparent in what we do. Our clients can } \\
\text { see all the changes that we are or aren't making, so we need to } \\
\text { make sure that whoever we hire has a lot of integrity and is } \\
\text { going to do the right thing. (In response to the prompt: "Tell me } \\
\text { about your star employees. Tell me about the people who } \\
\text { represent the best of your organization") }\end{array}$ & $\begin{array}{l}\text { Interview with } \\
\text { [Employee C] }\end{array}$ \\
\hline & $\begin{array}{l}\text { Integrity, owning up to mistakes, transparency, being real } \\
\text { ([Employee E]); Authenticity ([Employee E]); Authentic care - } \\
\text { personally \& professionally ([Employee E] LT); } \\
\text { Openess/transparency ([Employee F] LT); Integrity. We're } \\
\text { performance based. ([Employee C]); Transparency and honesty } \\
\text { ([Employee D] LT). (Written responses by Interviewees in } \\
\text { response to the following prompt: "Tell me about your star } \\
\text { employees. Tell me about the people who represent the best of } \\
\text { your organization." Written on the Interview } 1 \text { Worksheet) }\end{array}$ & $\begin{array}{l}\text { Interview } 1 \\
\text { Worksheet }\end{array}$ \\
\hline & $\begin{array}{l}\text { [Employee B] recounts a story of authenticity/"being real") and } \\
\text { generosity that began when the company was a struggling } \\
\text { startup: We snapped a budget, we had the margin, we were } \\
\text { still trying to figure out how to manage this, so we knew we } \\
\text { weren't going to be able to pay people enough to justify their } \\
\text { time. And we told them that, and they knew it. "We're } \\
\text { underpaying; we know this. Our goal is to get you to livable } \\
\text { wage quickly, and move beyond that. We want to be generous } \\
\text { with things and we can't be." So we've continued that, even } \\
\text { as their wages have caught up and gone probably beyond what } \\
\text { a lot of them would make somewhere else. }\end{array}$ & $\begin{array}{l}\text { Interview with } \\
\text { [Employee B] }\end{array}$ \\
\hline
\end{tabular}




\begin{tabular}{|c|c|c|}
\hline & $\begin{array}{l}\text { CEO recounted a story where personal integrity forced him to } \\
\text { completely overhaul the company: ... if you looked at revenue, it } \\
\text { looked great. If you stepped back from the business it was } \\
\text { terrible... we had started } 2011 \text { with } 100 \text { clients, we sold } \\
\text { throughout the year } 300 \text { clients, we ended the year with } 100 \\
\text { clients. So we churned everything we sold. We had different } \\
\text { clients there, we had maybe } 10 \text { core clients that had been with } \\
\text { us for awhile who - in reality (he laughs) were probably too } \\
\text { dumb to move on. So I saw that and realized, probably early } \\
2012, \text { we're just not going to sell anything. No more sales } \\
\text { team. If we can't produce a product that retains our business, } \\
\text { we don't have a product worth selling. And just personal } \\
\text { integrity in that said, 'I can't possibly endorse this'. }\end{array}$ & $\begin{array}{l}\text { Interview with } \\
\text { [Employee B] }\end{array}$ \\
\hline & $\begin{array}{l}\text { Talking about employees: because we're not locked in with } \\
\text { contracts, they have to have that desire to really do what's } \\
\text { right, do what's best. }\end{array}$ & $\begin{array}{l}\text { Interview with } \\
\text { [Employee C] }\end{array}$ \\
\hline & $\begin{array}{l}\text { We know the more we share with them, and the more open we } \\
\text { are with them, they know they can come in and talk with us } \\
\text { about anything and everything all the time. So that's important. } \\
\text { (describing a shared understanding of the leadership team) }\end{array}$ & $\begin{array}{l}\text { Interview with } \\
\text { [Employee C] }\end{array}$ \\
\hline & $\begin{array}{l}\text {...integrity, and honesty, and we try to be as transparent as we } \\
\text { can with our employees - so letting them know, here's the } \\
\text { changes that are happening or could be happening, or here's } \\
\text { what kind of what's going on behind the scenes. We try to be } \\
\text { as open with them as possible, because if you're not, that's } \\
\text { what kind of creates the chit chat, and then rumors start } \\
\text { spreading, and it just creates a lot of unrest. (describing values } \\
\text { of the leadership team and their impacts on company culture) }\end{array}$ & $\begin{array}{l}\text { Interview with } \\
\text { [Employee C] }\end{array}$ \\
\hline & $\begin{array}{l}\text { And then transparency and honesty I think... I mean I've } \\
\text { worked at places that don't have any; and it's such a difference. } \\
\text { (describing the leadership team) }\end{array}$ & $\begin{array}{l}\text { Interview with } \\
\text { [Employee D] }\end{array}$ \\
\hline & $\begin{array}{l}\text { Drama and Politics were missing from the senior management } \\
\text { team meeting. }\end{array}$ & Observation \\
\hline & $\begin{array}{l}\text { When you hear us talking to clients, that's who we are. I mean, } \\
\text { I think [Employee D] is the best example of that. I mean I love } \\
\text { his energy, and when he talks to clients he talks like he knows } \\
\text { what he's talking about - and he does - but he does it at such a } \\
\text { personal level and I really admire that. If you ever get a } \\
\text { chance, I would listen in to a conversation he has with his } \\
\text { clients. He's very personal, he's very 'this is who I am, so this is } \\
\text { what you get'. }\end{array}$ & $\begin{array}{l}\text { Interview with } \\
\text { [Employee E] }\end{array}$ \\
\hline $\begin{array}{l}\text { - Good } \\
\text { communication; } \\
\text { active listening. }\end{array}$ & $\begin{array}{l}\text { Star employees are also tend to be naturally very gifted in } \\
\text { communication (In response to the prompt: "Tell me about } \\
\text { your star employees. Tell me about the people who represent } \\
\text { the best of your organization") }\end{array}$ & $\begin{array}{l}\text { Interview with } \\
\text { [Employee B] }\end{array}$ \\
\hline
\end{tabular}




\begin{tabular}{|c|c|c|}
\hline & $\begin{array}{l}\text { I very very much try to make an upward flow of information in } \\
\text { our company. ... I always firmly believed that that was the best } \\
\text { way to do it, because those are the people doing the work, } \\
\text { they're producing the product, they're doing the service: } \\
\text { they're the ones that have the insight and the day to day on } \\
\text { what's next and they're going to have the best ideas, and so } \\
\text { you have to have a way for them to voice that }\end{array}$ & $\begin{array}{l}\text { Interview with } \\
\text { [Employee D] }\end{array}$ \\
\hline & $\begin{array}{l}\text { Listening. We really have to make sure that, as a leadership } \\
\text { team, that we're constantly listening. And not just listening to } \\
\text { kind of cross our t's and dot our i's, but actually hear them... } \\
\text { [and] we want all of our star employees to be leaders, so a lot } \\
\text { of it kind of crosses over. }\end{array}$ & $\begin{array}{l}\text { Interview with } \\
\text { [Employee C] }\end{array}$ \\
\hline & $\begin{array}{l}\text { Communication ([Employee B]); Listening. Active listening. } \\
\text { Intentional approach to listening. ([Employee C] LT); Vocal } \\
\text { (share thoughts, insights, etc) ([Employee E]); Communicating } \\
\text { what is best for the client ([Employee A]) (Written responses by } \\
\text { Interviewees in response to the following prompt: "Tell me } \\
\text { about your star employees. Tell me about the people who } \\
\text { represent the best of your organization." Written on the } \\
\text { Interview } 1 \text { Worksheet) }\end{array}$ & $\begin{array}{l}\text { Interview } 1 \\
\text { Worksheet }\end{array}$ \\
\hline \multicolumn{3}{|c|}{ Theme: Balance } \\
\hline \multirow[t]{3}{*}{$\begin{array}{l}\text { Balance. Our work } \\
\text { requires a balance } \\
\text { of both technical } \\
\text { prowess and } \\
\text { people skills. Both } \\
\text { are necessary, in } \\
\text { balance. }\end{array}$} & $\begin{array}{l}\text { Yeah... there's this unique kind of meshing of worlds, where we } \\
\text { have the science and we have the art. And what we do is a } \\
\text { combination of both. So we need that technology, we need } \\
\text { that science to do the job, because we have tons of data to } \\
\text { analyze, but we also need that art because we're dealing with } \\
\text { people in our marketing and our advertising. Not only with our } \\
\text { clients, but also who we're trying to meet and reach in our } \\
\text { advertising. (In response to the question: "Tell me, what is one } \\
\text { piece of advice you would give to a new employee or applicant } \\
\text { about how to thrive at this company?") }\end{array}$ & $\begin{array}{l}\text { Key Informant } \\
\text { Interview with } \\
\text { [Employee E] }\end{array}$ \\
\hline & $\begin{array}{l}\text { We just look at data so much, you have to be able to look at } \\
\text { data, and see patterns and make decisions on it very quickly. } \\
\text { Star employees are also tend to be naturally very gifted in } \\
\text { communication. And that's very rare to get both a math mind } \\
\text { and a communication mind. (In response to the prompt: "Tell } \\
\text { me about your star employees. Tell me about the people who } \\
\text { represent the best of your organization") }\end{array}$ & $\begin{array}{l}\text { Interview with } \\
\text { [Employee B] }\end{array}$ \\
\hline & $\begin{array}{l}\text { those to me are the two sides. It's really, you know, } \\
\text { communication with the client, your employees; and learning } \\
\text { our product and what we do and how we provide the services, } \\
\text { and the strategy and that kind of stuff behind it (describing } \\
\text { what it takes to succeed in the firm) }\end{array}$ & $\begin{array}{l}\text { Interview with } \\
\text { [Employee D] }\end{array}$ \\
\hline
\end{tabular}




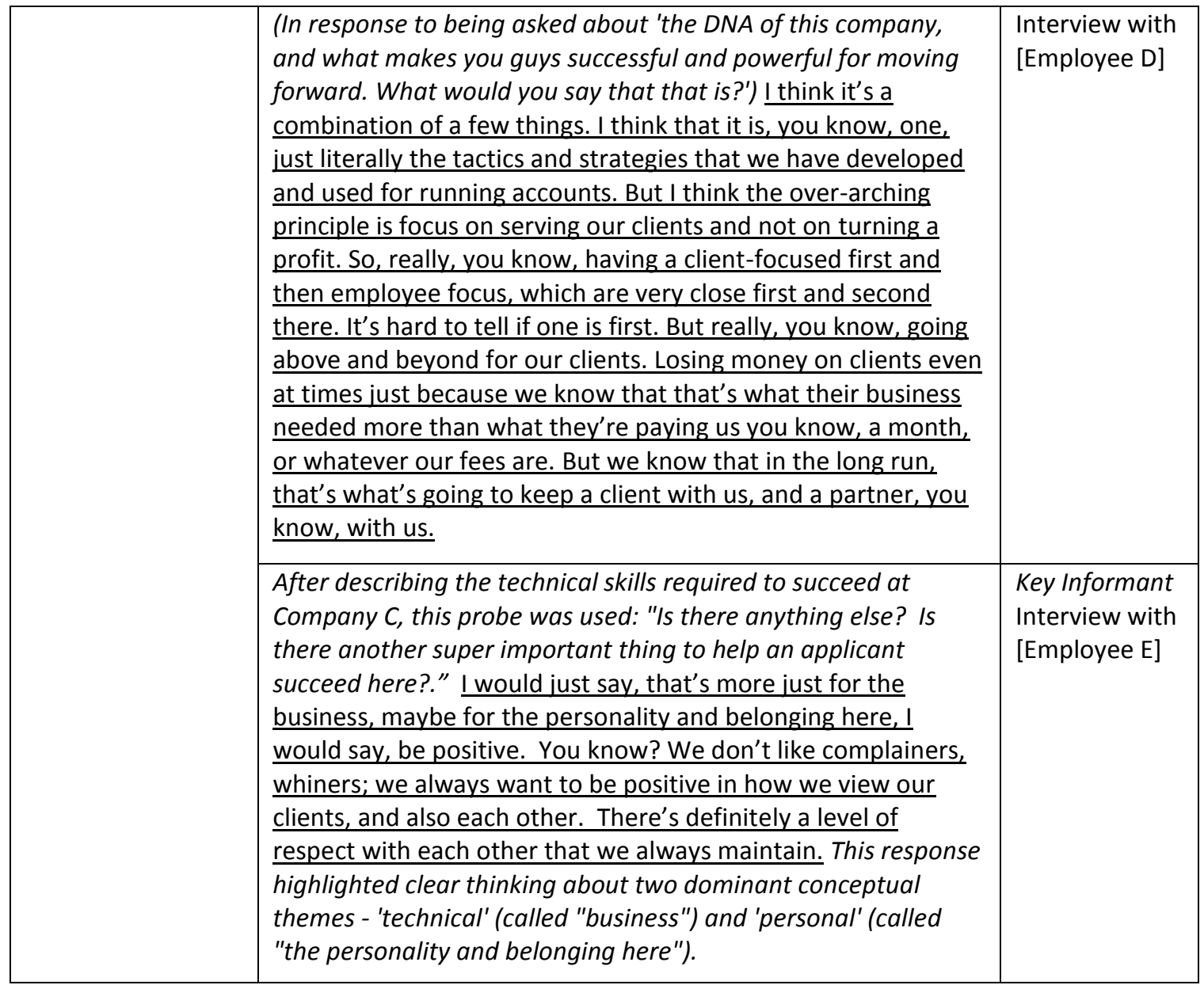

